\author{
AUS DEM LEHRSTUHL FÜR FRAUENHEILKUNDE UND GEBURTSHILFE \\ PROF. DR. MED. BIRGIT SEELBACH-GÖBEL \\ DER FAKULTÄT FÜR MEDIZIN \\ DER UNIVERSITÄT REGENSBURG
}

\title{
ADIPOSITAS IN DER SCHWANGERSCHAFT
}

Auswirkungen auf den Schwangerschafts-, Geburts- und Wochenbettverlauf sowie das kindliche Outcome

\author{
Inaugural - Dissertation \\ zur Erlangung des Doktorgrades \\ der Medizin \\ der \\ Fakultät für Medizin \\ der Universität Regensburg
}

vorgelegt von

Susanne Artus 



\author{
AUS DEM LEHRSTUHL FÜR FRAUENHEILKUNDE UND GEBURTSHILFE \\ PROF. DR. MED. BIRGIT SEELBACH-GÖBEL \\ DER FAKULTÄT FÜR MEDIZIN \\ DER UNIVERSITÄT REGENSBURG
}

\title{
ADIPOSITAS IN DER SCHWANGERSCHAFT
}

\author{
Auswirkungen auf den Schwangerschafts-, Geburts- und Wochenbettverlauf sowie das \\ kindliche Outcome
}

\author{
Inaugural - Dissertation \\ zur Erlangung des Doktorgrades \\ der Medizin \\ der \\ Fakultät für Medizin \\ der Universität Regensburg
}

vorgelegt von

Susanne Artus 
Dekan:

1. Berichterstatter:

2. Berichterstatter:

Tag der mündlichen Prüfung:
Prof. Dr. med. Dipl. Phys. Dirk Hellwig

Prof. Dr. med. Birgit Seelbach-Göbel

Prof. Dr. Iris Heid

27.05.2020 


\section{Inhaltsverzeichnis}

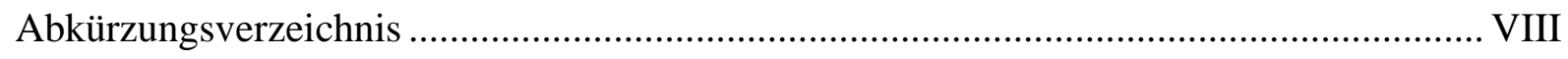

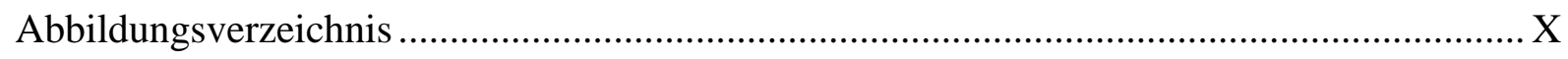

Tabellenverzeichnis................................................................................................. XII

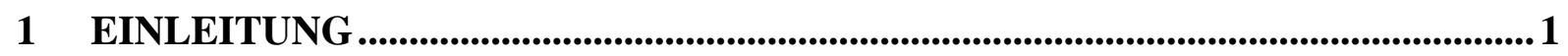

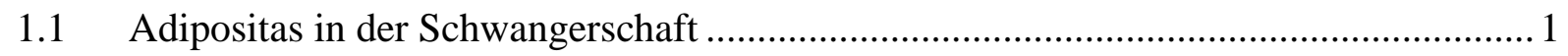

1.1.1 Krankheitsbild der Adipositas ...................................................................... 1

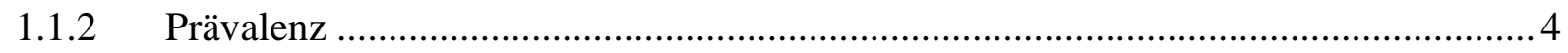

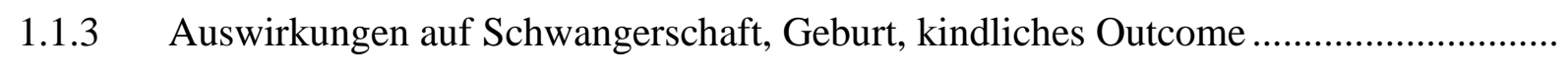

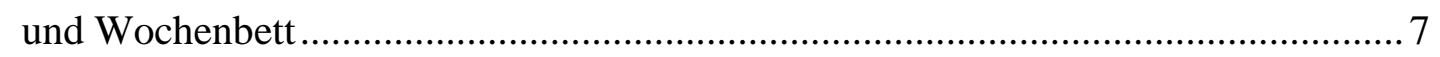

1.1.4 Langzeitwirkungen durch Perinatale Programmierung ........................................ 12

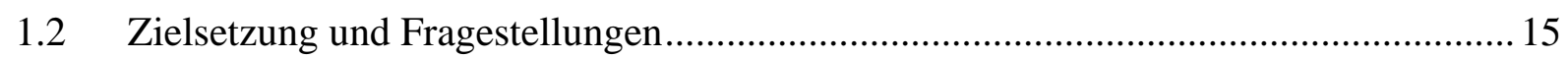

2 PATIENTENKOLLEKTIV UND METHODIK ..................................................

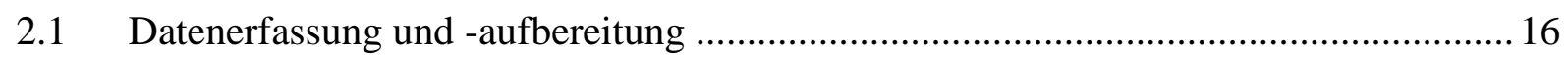

2.2 Auswahlkriterien und Beschreibung des Studienkollektivs ..................................... 16

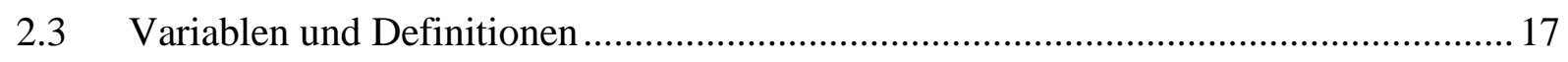

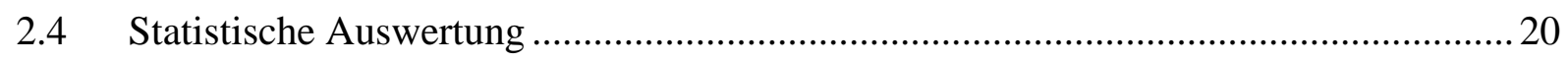

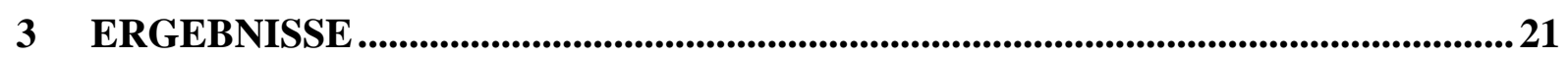

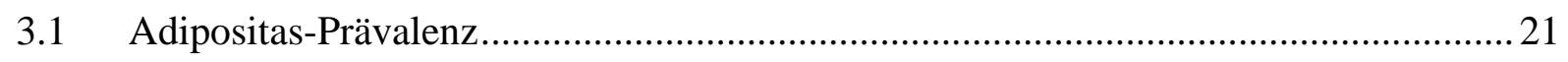

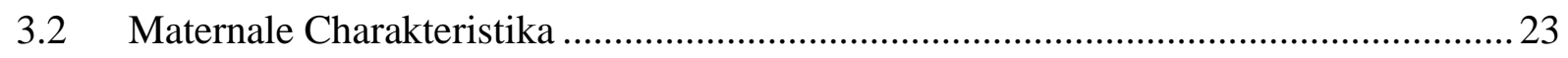

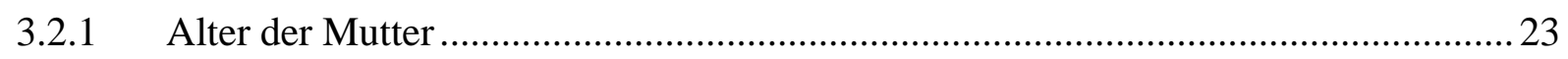

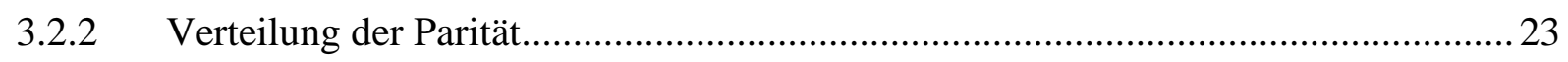

3.2.3 Aborte in früheren Schwangerschaften .......................................................... 24

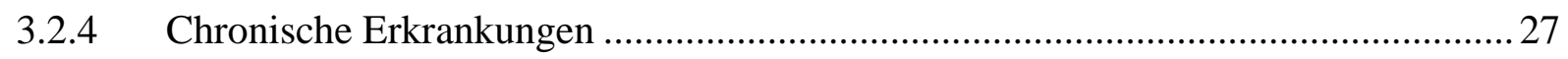

3.2.5 Gewichtszunahme während der Schwangerschaft ...............................................29 


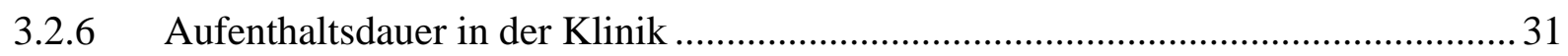

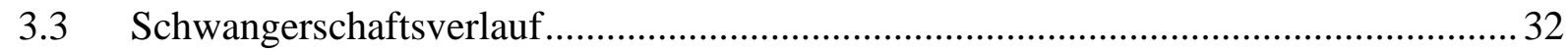

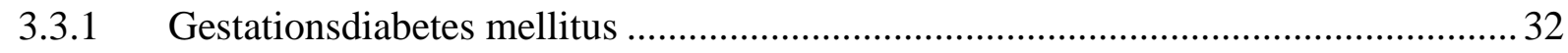

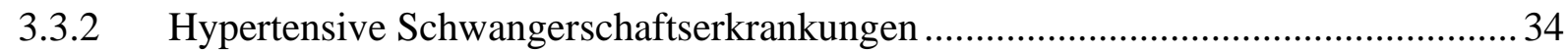

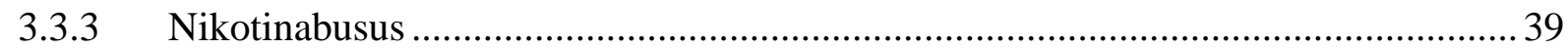

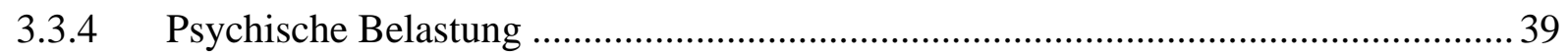

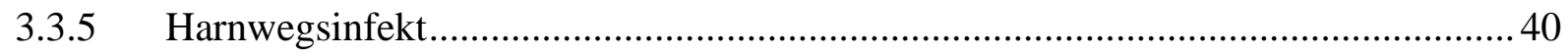

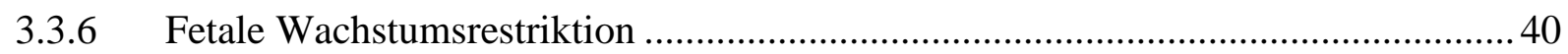

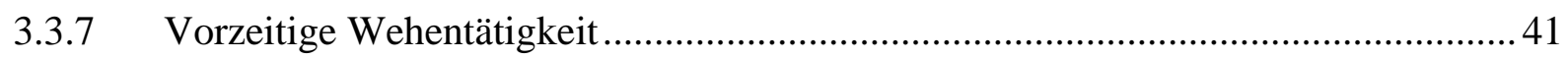

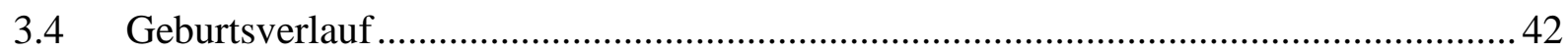

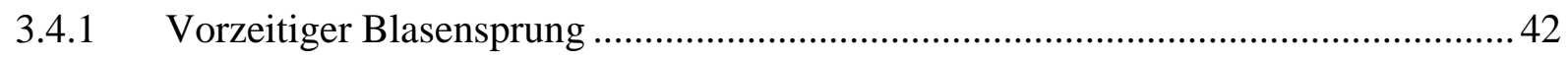

3.4.2 Geburtsmodus und Indikationen zur Sectio caesarea ......................................... 42

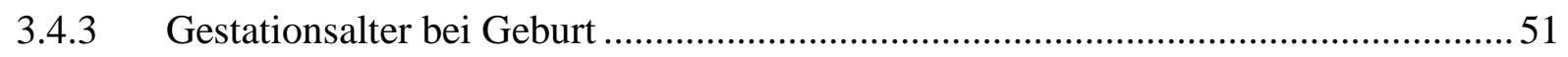

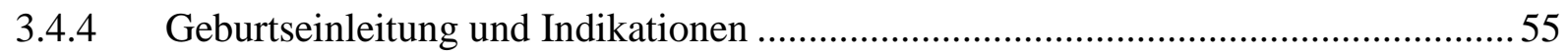

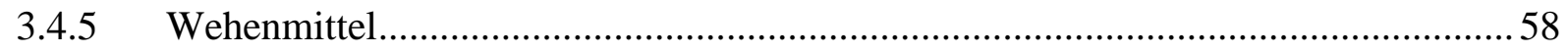

3.4.6 Schulterdystokie und resultierende kindliche Geburtsverletzungen ........................60

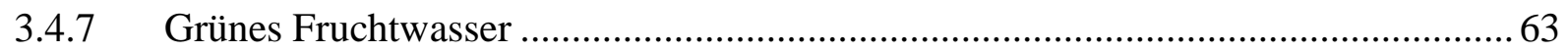

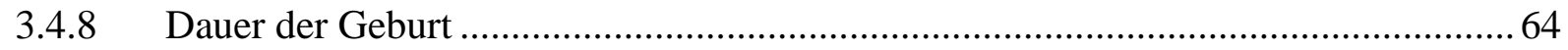

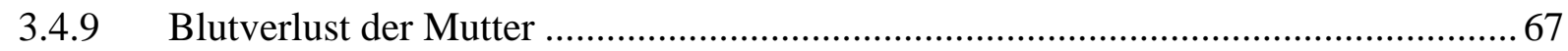

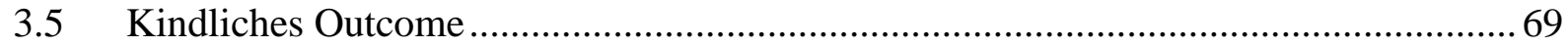

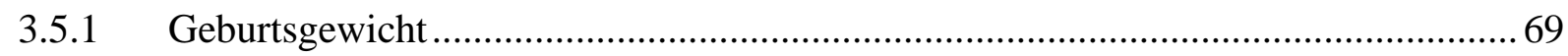

3.5.2 Apgar-Werte nach einer, fünf und zehn Minuten postnatal .................................. 72

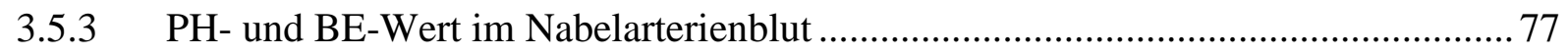

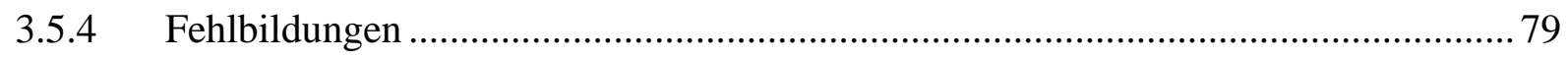

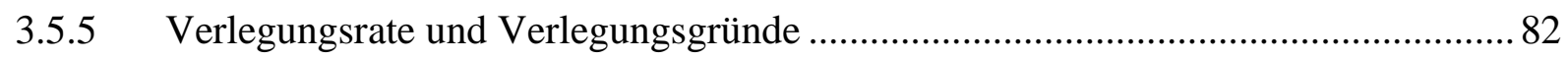

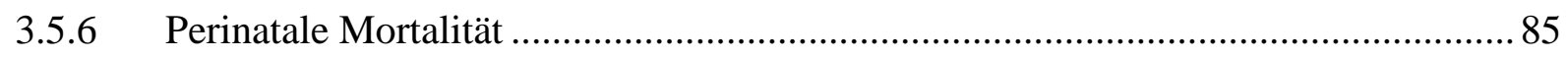




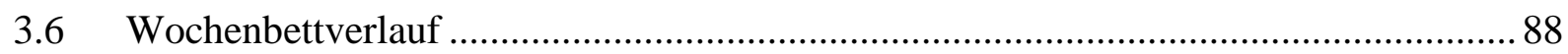

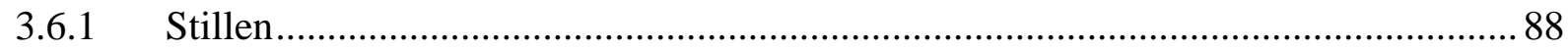

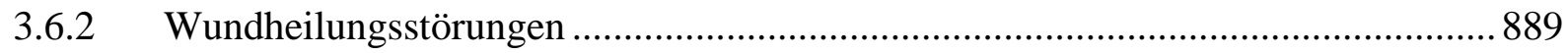

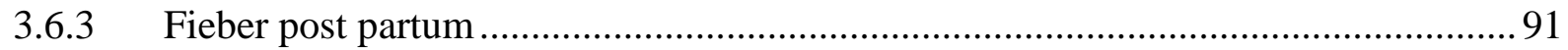

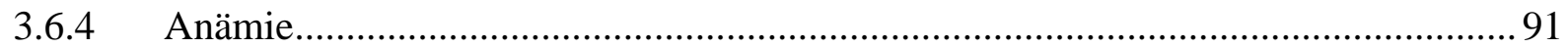

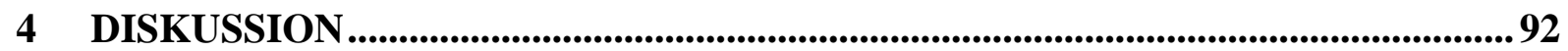

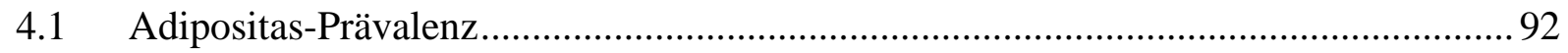

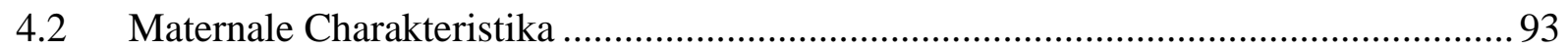

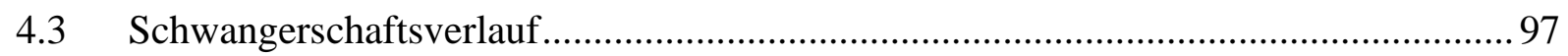

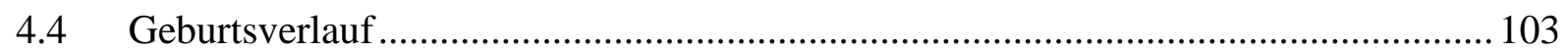

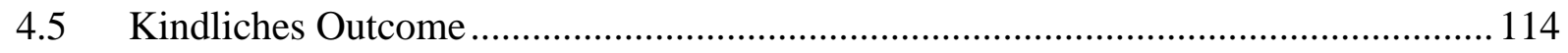

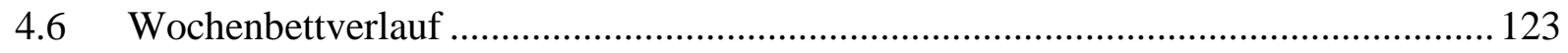

4.7 Prävention durch gesunde Ernährung und Sport in der Schwangerschaft .................. 126

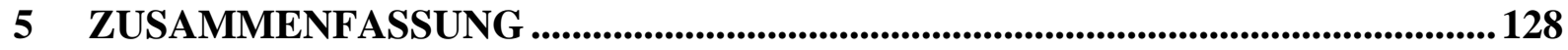

6 LITERATURVERZEICHNIS........................................................................................... 129

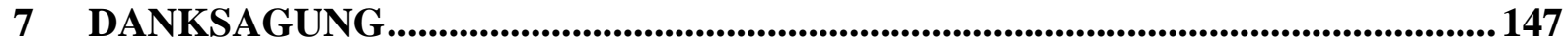

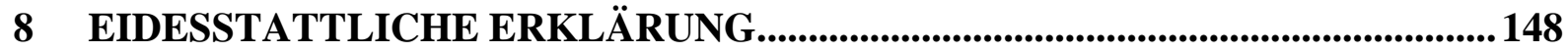




\section{Abkürzungsverzeichnis}

\begin{tabular}{|c|c|}
\hline ANOVA & Analysis of variance between groups \\
\hline $\mathbf{a O R}$ & Adjustiertes Odds Ratio \\
\hline $\mathbf{A P}$ & Austreibungsperiode der Geburt \\
\hline Apgar & Aussehen, Puls, Grimassen, Aktivität, Respiration \\
\hline BAQ & $\begin{array}{l}\text { Bayerische Arbeitsgemeinschaft für Qualitätssicherung in der stationä- } \\
\text { ren Versorgung }\end{array}$ \\
\hline BE & Base Excess \\
\hline BEL & Beckenendlage \\
\hline BMI & Body Mass Index $\left(\mathrm{kg} / \mathrm{m}^{2}\right)$ \\
\hline BSG98 & Bundes-Gesundheitssurvey 1998 \\
\hline cffDNA & Cell-free fetal DNA \\
\hline CTG & Cardiotokogramm \\
\hline DEGS1 & Studie zur Gesundheit Erwachsener in Deutschland \\
\hline $\mathbf{E P}$ & Eröffnungsperiode der Geburt \\
\hline FeLIPO & $\begin{array}{l}\text { Feasibility of a lifestyle intervention programme to prevent obesity in the } \\
\text { offspring }\end{array}$ \\
\hline GDM & Gestationsdiabetes mellitus \\
\hline GeliS & Gesund leben in der Schwangerschaft \\
\hline HAPO & Hyperglycaemia and Adverse Pregnancy Outcome \\
\hline HDL & High Density Lipoprotein \\
\hline HELLP & Hemolysis, Elevated Liver enzymes, Low Platelets \\
\hline IOM & Institute of Medicine \\
\hline IUFT & Intrauteriner Fruchttod \\
\hline IUGR & Intrauterine growth restriction \\
\hline
\end{tabular}




\begin{tabular}{|c|c|}
\hline KErn & Kompetenzzentrum für Ernährung \\
\hline LDL & Low Density Lipoprotein \\
\hline LGA & Large-for-Gestational-Age \\
\hline LSD & Least significant difference \\
\hline $\mathbf{N}$ & Stichprobengröße \\
\hline $\mathbf{n}$ & Größe einer Teilstichprobe \\
\hline NIPT & Nicht-invasives pränatales Testing \\
\hline OECD & Organisation for Economic Cooperation and Development \\
\hline OR & Odds Ratio \\
\hline PPH & Peripartale Hämorrhagie \\
\hline PStV & Personenstandsverordnung \\
\hline $\mathbf{R R}$ & Relatives Risiko \\
\hline SD & Standardabweichung \\
\hline SGA & Small-for-Gestational-Age \\
\hline SPSS & Statistical Product and Service Solutions \\
\hline SL & Schädellage \\
\hline SSW & Schwangerschaftswoche \\
\hline V. a. & Verdacht auf \\
\hline WHO & World Health Organization \\
\hline Z. $\mathbf{n}$. & Zustand nach \\
\hline
\end{tabular}




\section{Abbildungsverzeichnis}

Abb. 1 Relative Häufigkeit von Adipositas in der Schwangerschaft in Bayern und Deutschland von 2005 bis 2015

Abb. 2 Relative Häufigkeit von Adipositas in der Schwangerschaft und GDM in Bayern von 2000 bis 2015

Abb. 3 Allgemeines Konzept zur Perinatalen Programmierung eines erhöhten Adipositasund Diabetesrisikos nach Schellong 2008 (133)

Abb. 4 Relative Häufigkeitsverteilung der verschiedenen maternalen BMI-Kategorien im Studienkollektiv (N=29180).

Abb. 5 Absolute Häufigkeiten der verschiedenen maternalen BMI-Kategorien pro Jahr von 2000-2015 ( $\mathrm{N}=29180)$

Abb. 6 Relative Häufigkeitsverteilung von Primi- und Pluriparae in Bezug auf maternale BMI-Kategorien $(\mathrm{N}=27754)$.

Abb. 7 Relative Häufigkeitsverteilung der klassifizierten Anzahl stattgehabter Aborte in früheren Schwangerschaften in Bezug auf maternale BMI-Kategorien $(\mathrm{N}=27754)$.... 25

Abb. 8 Relative Häufigkeitsverteilung des präexistenten Diabetes mellitus in Bezug auf maternale BMI-Kategorien $(\mathrm{N}=27754)$ 28

Abb. 9 Relative Häufigkeitsverteilung der Einhaltung sowie Unter- und Überschreitung der empfohlenen Gewichtszunahme des IOM in Bezug auf maternale BMI-Kategorien $(\mathrm{N}=26232)$. 30

Abb. 10 Relative Häufigkeitsverteilung des Gestationsdiabetes mellitus in Bezug auf maternale BMI-Kategorien ( $\mathrm{N}=27412)$

Abb. 11 Relative Häufigkeitsverteilung der Präeklampsie in Bezug auf maternale BMIKategorien $(\mathrm{N}=27754)$

Abb. 12 Relative Häufigkeitsverteilung der verschiedenen Geburtsmodi in Bezug auf maternale BMI-Kategorien $(\mathrm{N}=27747)$ 
Abb. 13 Relative Häufigkeitsverteilung des in Zeiträume klassifizierten Gestationsalters in Bezug auf maternale BMI-Kategorien $(\mathrm{N}=27737)$

Abb. 14 Relative Häufigkeitsverteilung der Geburtseinleitung in Bezug auf maternale BMI-Kategorien $(\mathrm{N}=27754)$

Abb. 15 Relative Häufigkeitsverteilung des Einsatzes eines Wehenmittels unter der Geburt in Bezug auf maternale BMI-Kategorien $(\mathrm{N}=24518)$

Abb. 16 Relative Häufigkeitsverteilung von grünem Fruchtwasser in Bezug auf maternale BMI-Kategorien $(\mathrm{N}=27754)$

Abb. 17 Kindliches Geburtsgewicht [g] in Bezug auf maternale BMI-Kategorien $(\mathrm{N}=27745)$ 70

Abb. 18 Relative Häufigkeitsverteilung der klassifizierten kindlichen

Geburtsgewichtsperzentile in Bezug auf maternale BMI-Kategorien (N=27700). 71

Abb. 19 Relative Häufigkeitsverteilung der klassifizierten Apgar-Werte nach der ersten Lebensminute in Bezug auf maternale BMI-Kategorien $(\mathrm{N}=27540)$ 75

Abb. 20 Relative Häufigkeitsverteilung der klassifizierten Apgar-Werte nach der fünften Lebensminute in Bezug auf maternale BMI-Kategorien $(\mathrm{N}=27540)$ 76

Abb. 21 Relative Häufigkeitsverteilung der klassifizierten Apgar-Werte nach der zehnten Lebensminute in Bezug auf maternale BMI-Kategorien $(\mathrm{N}=27540)$ .76

Abb. 22 Relative Häufigkeitsverteilung des klassifizierten kindlichen pH-Werts im Nabelarterienblut in Bezug auf mütterliche BMI-Kategorien (N=27492). .78

Abb. 23 Relative Häufigkeitsverteilung einer kindlichen Verlegung auf die neonatologische Station in Bezug auf maternale BMI-Kategorien $(\mathrm{N}=27618)$ 82

Abb. 24 Relative Häufigkeitsverteilung der verschiedenen Formen des Stillverhaltens der Wöchnerinnen in Bezug auf deren BMI-Kategorien ( $\mathrm{N}=23672)$. .89 


\section{Tabellenverzeichnis}

Tab. 1 Gewichtsklassifikation anhand des Body Mass Index nach Angaben der WHO (3) ..... 2

Tab. 2 Relatives Risiko für die Entwicklung von Komorbiditäten der Adipositas nach Angaben der WHO (3) 3

Tab. 3 Absolute und relative Häufigkeiten der maternalen BMI-Kategorien pro Jahr von 2000 bis 2015

Tab. 4 Mittelwert und Standardabweichung des mütterlichen Alters bei Entbindung in Bezug auf BMI-Kategorien

Tab. 5 Absolute und relative Häufigkeiten von Primi- und Pluriparae in Bezug auf maternale BMI-Kategorien

Tab. 6 Absolute und relative Häufigkeit der klassifizierten Anzahl stattgehabter Aborte in früheren Schwangerschaften in Bezug auf maternale BMI-Kategorien

Tab. 7 Absolute und relative Häufigkeiten der klassifizierten Anzahl stattgehabter Aborte in früheren Schwangerschaften in Bezug auf maternale BMI-Kategorien in der Subgruppe stoffwechselgesunder Frauen 26

Tab. 8 Absolute und relative Häufigkeiten eines präexistenten Diabetes mellitus in Bezug auf maternale BMI-Kategorien.

Tab. 9 Absolute und relative Häufigkeiten einer chronischen Hypertonie in Bezug auf maternale BMI-Kategorien

Tab. 10 Mittelwert und Standardabweichung der maternalen Gewichtszunahme während der Schwangerschaft in Bezug auf BMI-Kategorien.

Tab. 11 Absolute und relative Häufigkeiten einer Einhaltung sowie Unter- und Überschreitung der empfohlenen Gewichtszunahme des IOM in Bezug auf maternale BMI-Kategorien

Tab. 12 Mittelwert und Standardabweichung der mütterlichen Aufenthaltsdauer in der

Klinik insgesamt in Bezug auf BMI-Kategorien. 
Tab. 13 Absolute und relative Häufigkeiten eines Gestationsdiabetes mellitus in Bezug auf maternale BMI-Kategorien

Tab. 14 Absolute und relative Häufigkeiten eines Gestationsdiabetes mellitus in Bezug auf maternale BMI-Kategorien, stratifiziert nach mütterlichen Altersgruppen

Tab. 15 Absolute und relative Häufigkeiten einer Gestationshypertonie in Bezug auf maternale BMI-Kategorien

Tab. 16 Absolute und relative Häufigkeiten einer Präeklampsie in Bezug auf maternale BMI-Kategorien

Tab. 17 Absolute und relative Häufigkeiten eines HELLP-Syndroms in Bezug auf maternale BMI-Kategorien.

Tab. 18 Absolute und relative Häufigkeiten einer Eklampsie in Bezug auf maternale

BMI-Kategorien

Tab. 19 Absolute und relative Häufigkeiten einer Präeklampsie in Bezug auf maternale BMI-Kategorien, stratifiziert nach Parität 38

Tab. 20 Absolute und relative Häufigkeiten einer Präeklampsie in Bezug auf maternale BMI-Kategorien in der Subgruppe stoffwechselgesunder Frauen 38

Tab. 21 Absolute und relative Häufigkeiten eines Nikotinabusus während der

Schwangerschaft in Bezug auf maternale BMI-Kategorien 39

Tab. 22 Absolute und relative Häufigkeiten einer besonderen psychischen Belastung während der Schwangerschaft in Bezug auf maternale BMI-Kategorien

Tab. 23 Absolute und relative Häufigkeiten eines Harnwegsinfekts in Bezug auf maternale BMI-Kategorien

Tab. 24 Absolute und relative Häufigkeiten eines IUGR in Bezug auf maternale BMIKategorien 40

Tab. 25 Absolute und relative Häufigkeiten der vorzeitigen Wehentätigkeit in Bezug auf maternale BMI-Kategorien. 
Tab. 26 Absolute und relative Häufigkeiten eines vorzeitigen Blasensprungs in Bezug auf maternale BMI-Kategorien.

Tab. 27 Absolute und relative Häufigkeiten der verschiedenen Geburtsmodi in Bezug auf maternale BMI-Kategorien

Tab. 28 Absolute und relative Häufigkeiten einer Beckenendlage gesamt in Bezug auf maternale BMI-Kategorien

Tab. 29 Absolute und relative Häufigkeiten der Sectio caesarea in Bezug auf maternale BMI-Kategorien, stratifiziert nach Parität.

Tab. 30 Häufigkeit der sekundären Sectio in Bezug auf mütterliche BMI-Kategorien, stratifiziert nach dem Vorliegen einer Geburtseinleitung .46

Tab. 31 Absolute und relative Häufigkeiten einer Sectio caesarea in Bezug auf maternale BMI-Kategorien nach Ausschluss der Schwangeren mit adipositas-assoziierten Komorbiditäten 46

Tab. 32 Absolute und relative Häufigkeiten der verschiedenen Indikationen zur primären Sectio in Bezug auf maternale BMI-Kategorien 48

Tab. 33 Absolute und relative Häufigkeiten der verschiedenen Indikationen zur sekundären Sectio in Bezug auf maternale BMI-Kategorien 50

Tab. 34 Absolute und relative Häufigkeiten einer Not-Sectio in Bezug auf maternale BMI-Kategorien

Tab. 35 Absolute und relative Häufigkeiten des in Zeiträume klassifizierten

Gestationsalters in Bezug auf maternale BMI-Kategorien

Tab. 36 Absolute und relative Häufigkeiten des in Zeiträume klassifizierten Gestationsalters in Bezug auf maternale BMI-Kategorien nach Ausschluss der Schwangeren mit Diabetes mellitus oder einer hypertensiven Schwangerschaftserkrankung.

Tab. 37 Absolute und relative Häufigkeiten einer Geburtseinleitung in Bezug auf maternale BMI-Kategorien .55 
Tab. 38 Absolute und relative Häufigkeiten einer Geburtseinleitung in Bezug auf maternale BMI-Kategorien, stratifiziert nach Parität

Tab. 39 Absolute und relative Häufigkeiten einer Geburtseinleitung in Bezug auf maternale BMI-Kategorien nach Ausschluss der Frauen mit Diabetes mellitus oder hypertensiven Erkrankungen in der Schwangerschaft

Tab. 40 Absolute und relative Häufigkeiten der verschiedenen Indikationen zur

Geburtseinleitung in Bezug auf maternale BMI-Kategorien

Tab. 41 Absolute und relative Häufigkeiten des Einsatzes eines Wehenmittels unter der

Geburt in Bezug auf maternale BMI-Kategorien .....

Tab. 42 Absolute und relative Häufigkeiten einer Schulterdystokie in Bezug auf maternale

BMI-Kategorien 60

Tab. 43 Absolute und relative Häufigkeiten einer Schulterdystokie in Bezug auf maternale BMI-Kategorien nach Ausschluss der Frauen mit Diabetes mellitus, einer vaginaloperativen Entbindung und der Geburt eines LGA-Kindes

Tab. 44 Absolute und relative Häufigkeiten einer Schulterdystokie in Bezug auf maternale BMI-Kategorien, stratifiziert nach Parität

Tab. 45 Mütterliche bzw. kindliche Charakteristika der verschiedenen Fälle einer kindlichen Geburtsverletzung infolge einer Schulterdystokie

Tab. 46 Absolute und relative Häufigkeiten von grünem Fruchtwasser in Bezug auf maternale BMI-Kategorien.

Tab. 47 Mittlere Geburtsdauer und SD der vaginalen Entbindung aus SL unter Primiparae in Bezug auf maternale BMI-Kategorien

Tab. 48 Mittlere Geburtsdauer und SD der vaginalen Entbindung aus SL unter Pluriparae in Bezug auf maternale BMI-Kategorien

Tab. 49 Absolute und relative Häufigkeiten einer protrahierten EP in Bezug auf maternale BMI-Kategorien .66 
Tab. 50 Absolute und relative Häufigkeiten eines Geburtsstillstands in der EP in Bezug auf maternale BMI-Kategorien.

Tab. 51 Absolute und relative Häufigkeiten einer protrahierten AP in Bezug auf maternale BMI-Kategorien 66

Tab. 52 Absolute und relative Häufigkeiten eines Geburtsstillstands in der EP in Bezug auf maternale BMI-Kategorien.

Tab. 53 Mittelwert und SD des Blutverlustes der Mütter unter der Geburt in Bezug auf deren BMI-Kategorien.

Tab. 54 Absolute und relative Häufigkeiten einer PPH nach vaginaler Geburt in Bezug auf maternale BMI-Kategorien.

Tab. 55 Absolute und relative Häufigkeiten einer PPH nach Sectio caesarea in Bezug auf maternale BMI-Kategorien.

Tab. 56 Mittelwert und SD des kindlichen Geburtsgewichts in Bezug auf maternale BMI-

Kategorien

Tab. 57 Absolute und relative Häufigkeiten der klassifizierten kindlichen

Geburtsgewichtsperzentile in Bezug auf maternale BMI-Kategorien. 71

Tab. 58 Absolute und relative Häufigkeiten eines LGA-Kindes in Bezug auf maternale BMI-Kategorien in der Subgruppe stoffwechselgesunder Frauen .72

Tab. 59 Mittelwerte und SD des kindlichen Apgar-Werts nach einer, fünf und zehn Lebensminuten in Bezug auf maternale BMI-Kategorien. .73

Tab. 60 Absolute und relative Häufigkeiten der klassifizierten Apgar-Werte nach der ersten Lebensminute in Bezug auf maternale BMI-Kategorien. .74

Tab. 61 Absolute und relative Häufigkeiten der klassifizierten Apgar-Werte nach der fünften Lebensminute in Bezug auf maternale BMI-Kategorien.... .74

Tab. 62 Absolute und relative Häufigkeiten der klassifizierten Apgar-Werte nach der zehnten Lebensminute in Bezug auf maternale BMI-Kategorien 
Tab. 63 Mittelwert und SD des kindlichen pH-Werts im Nabelarterienblut in Bezug auf maternale BMI-Kategorien

Tab. 64 Absolute und relative Häufigkeiten des klassifizierten kindlichen pH-Werts im Nabelarterienblut in Bezug auf maternale BMI-Kategorien

Tab. 65 Mittelwert und SD des kindlichen BE-Werts im Nabelarterienblut in Bezug auf maternale BMI-Kategorien

Tab. 66 Absolute und relative Häufigkeiten einer kindlichen Fehlbildung in Bezug auf maternale BMI-Kategorien

Tab. 67 Absolute und relative Häufigkeiten einer pränatalen Diagnose unter allen fehlgebildeten Kindern in Bezug auf maternale BMI-Kategorien

Tab. 68 Absolute und relative Häufigkeiten der verschiedenen Arten einer kindlichen

Fehlbildung in Bezug auf maternale BMI-Kategorien

Tab. 69 Absolute und relative Häufigkeiten einer kindlichen Verlegung auf die neonatologische Station in Bezug auf maternale BMI-Kategorien 82

Tab. 70 Absolute und relative Häufigkeiten der verschiedenen Indikationen zur kindlichen Verlegung auf die neonatologische Station in Bezug auf maternale BMI-Kategorien 84

Tab. 71 Absolute und relative Häufigkeiten der perinatalen Mortalität in Bezug auf maternale BMI-Kategorien

Tab. 72 Absolute und relative Häufigkeiten eines intrauterinen Fruchttodes in Bezug auf maternale BMI-Kategorien.

Tab. 73 Absolute und relative Häufigkeiten eines subpartualen Todes in Bezug auf maternale BMI-Kategorien 86

Tab. 74 Absolute und relative Häufigkeiten eines neonatalen Todes innerhalb der ersten sieben Lebenstage in Bezug auf maternale BMI-Kategorien 
Tab. 75 Absolute und relative Häufigkeiten der perinatalen Mortalität in Bezug auf das

Gestationsalter

Tab. 76 Absolute und relative Häufigkeiten der verschiedenen Formen des Stillverhaltens der Wöchnerinnen in Bezug auf deren BMI-Kategorien

Tab. 77 Absolute und relative Häufigkeiten einer revisionsbedürftigen

Wundheilungsstörung Bezug auf maternale BMI-Kategorien .90

Tab. 78 Absolute und relative Häufigkeiten einer Wundinfektion nach Sectio in Bezug auf maternale BMI-Kategorien... 90

Tab. 79 Absolute und relative Häufigkeiten von postpartalem Fieber in Bezug auf maternale BMI-Kategorien. 91

Tab. 80 Absolute und relative Häufigkeiten einer Anämie im Wochenbett in Bezug auf maternale BMI-Kategorien. .91 


\section{EINLEITUNG}

\subsection{Adipositas in der Schwangerschaft}

Adipositas gilt als Volkskrankheit des 21. Jahrhunderts. Die Prävalenz ist in den letzten Jahren stark angestiegen. Auch Frauen im gebärfähigen Alter sind von der globalen Epidemie betroffen, was zur Folge hat, dass immer mehr Frauen zu Beginn der Schwangerschaft einen BMI $\geq 30 \mathrm{~kg} / \mathrm{m}^{2}$ aufweisen. Maternale Adipositas birgt Risiken für Mutter und Kind während der Schwangerschaft, der Geburt und im Wochenbett und prägt den sich entwickelnden Fetus bereits intrauterin für ein erhöhtes Adipositas-Risiko im späteren Leben. Es ist von zentraler Bedeutung, ein Bewusstsein für die negativen Auswirkungen von Adipositas in der Schwangerschaft zu schaffen, wozu die vorliegende Arbeit einen Beitrag leisten soll.

\subsubsection{Krankheitsbild der Adipositas}

Adipositas, auch Fettleibigkeit genannt, ist definiert als eine Erhöhung des Körpergewichts durch eine über das Normalmaß hinausgehende Vermehrung des Körperfettanteils. Als international etablierte Beurteilungsgrundlage zur Klassifikation von Adipositas dient der sog. Body Mass Index (BMI). Dieser wird als Quotient aus Körpergewicht und Körpergröße zum Quadrat berechnet und in der Einheit $\left[\mathrm{kg} / \mathrm{m}^{2}\right]$ angegeben $(1,2)$.

$$
B M I=\frac{\text { Körpergewicht in Kilogramm }}{(\text { Körpergröße in Metern })^{2}}
$$

Nach der Klassifikation der World Health Organization (WHO) gilt eine Person als adipös, wenn der BMI einen Wert größer oder gleich $30 \mathrm{~kg} / \mathrm{m}^{2}$ aufweist. Zudem definiert die WHO weitere, auf dem BMI beruhende Gewichtskategorien und gibt davon ausgehend eine Einschätzung des Risikos für adipositas-assoziierte Folgeerkrankungen an (Tab. 1) (3). 
Tab. 1 Gewichtsklassifikation anhand des Body Mass Index nach Angaben der WHO (3)

\begin{tabular}{|c|c|c|}
\hline Kategorie & BMI $\left[\mathrm{kg} / \mathrm{m}^{2}\right]$ & Risiko für Folgeerkrankungen \\
\hline \hline Untergewicht & $<18,5$ & Niedrig \\
\hline Normalgewicht & $18,5-24,9$ & Durchschnittlich \\
\hline Übergewicht (Präadipositas) & $25,0-29,9$ & Gering erhöht \\
\hline Adipositas Grad I & $30,0-34,9$ & Erhöht \\
\hline Adipositas Grad II & $35,0-39,9$ & Hoch \\
\hline Adipositas Grad III & $\geq 40$ & Sehr hoch \\
\hline
\end{tabular}

Als Grundlage der Adipositas wird eine Störung der Regulation der Körperenergiehomöostase im Sinne eines Ungleichgewichts zwischen Kalorienzufuhr und körpereigenem Energieverbrauch angesehen. Die Deutsche Adipositas Gesellschaft nennt eine Interaktion u. a. folgender biologischer, psychosozialer und umweltbedingter Faktoren als Ursache für Übergewicht und Adipositas (2):

- Familiäre/genetische Disposition

- Lebensstil (z.B. Bewegungsmangel, Über- und Fehlernährung)

- Schlafmangel, chronischer Stress

- Depressive Erkrankungen

- Niedriger Bildungsstand und Sozialstatus

- Essstörungen (z.B. Binge-Eating-Störung, Night-Eating-Syndrom)

- Endokrine Erkrankungen (z.B. Hypothyreose, Cushing-Syndrom)

- Medikamente (z.B. Antidepressiva, Glukokortikoide, Antidiabetika, Antiepileptika)

Das Risiko für die Entwicklung von Begleit- und Folgeerkrankungen der Fettleibigkeit steigt laut WHO mit zunehmendem BMI an (3). Präadipositas und Adipositas werden als die bedeutendsten Risikofaktoren für die Entstehung eines metabolischen Syndroms angesehen, welches das Risiko für kardiovaskuläre und metabolische Erkrankungen deutlich erhöht $(1,2,4)$. Ein metabolisches Syndrom liegt laut der Definition der American Heart Association bzw. des National Heart, Lung and Blood Institutes bei einer Kombination von mindestens drei der im Folgenden dargestellten Risikofaktoren vor (5): 
- Taillenumfang: Männer $\geq 102 \mathrm{~cm}$, Frauen $\geq 88 \mathrm{~cm}$

- Triglyzeride: $\geq 150 \mathrm{mg} / \mathrm{dl}$ oder bereits eingeleitete Medikation

- HDL-Cholesterin: Männer <40 mg/dl, Frauen <50 mg/dl oder Medikation

- Blutdruck: systolisch $\geq 130 \mathrm{mmHg}$, diastolisch $\geq 85 \mathrm{mmHg}$ oder Medikation

- Nüchternplasmaglukose: $\geq 100 \mathrm{mg} / \mathrm{dl}$ oder Medikation

Fettleibigkeit kann auf verschiedene Organe und Organsysteme einen negativen Effekt ausüben. Ein BMI $\geq 30 \mathrm{~kg} / \mathrm{m}^{2}$ erhöht das Risiko u. a. für bestimmte Krebsarten wie Brust-, Endometrium- oder Kolonkarzinome, Herz-Kreislauf-Erkrankungen und für Diabetes mellitus Typ II. Tabelle 2 gibt - basierend auf Angaben der WHO - einen Überblick über das jeweilige relative Risiko, eine adipositas-assoziierte Komorbidität zu entwickeln (3).

Tab. 2 Relatives Risiko für die Entwicklung von Komorbiditäten der Adipositas nach Angaben der WHO (3)

\begin{tabular}{|c|c|c|}
\hline Risiko 1-2 -fach erhöht & Risiko 2-3 -fach erhöht & Risiko >3 -fach erhöht \\
\hline \hline $\begin{array}{c}\text { Karzinome: u. a. Brust (postmenopausal), } \\
\text { Endometrium, Kolon }\end{array}$ & Koronare Herzkrankheit & Diabetes Mellitus Typ II \\
\hline $\begin{array}{c}\text { Störung der Reproduktionshormone: } \\
\text { u. a. Hyperandrogenämie }\end{array}$ & Hypertonie & Cholezystolithiasis \\
\hline Polyzystisches Ovarsyndrom & $\begin{array}{c}\text { Arthrose } \\
\text { (v. a. Kniegelenke) }\end{array}$ & Dyslipidämie \\
\hline Unfruchtbarkeit & Hyperurikämie & Insulinresistenz \\
\hline Rückenschmerzen & Gicht & Fettleber \\
\hline Fetopathie & Refluxösophagitis & Schlaf-Apnoe \\
\hline
\end{tabular}

In der Folge ist die Mortalität von adipösen Menschen gegenüber Normalgewichtigen erhöht, was die Ergebnisse einer Metaanalyse von 57 prospektiven Studien der sog. „Prospective Studies Collaboration“ verdeutlichten. Bei den 900000 Studienteilnehmern war die mediane Lebenserwartung bei einem BMI zwischen $30,0 \mathrm{~kg} / \mathrm{m}^{2}$ und 35,0 kg/m² um zwei bis vier Jahre reduziert und bei einem BMI zwischen $40,0 \mathrm{~kg} / \mathrm{m}^{2}$ und 45,0 kg/m² um acht bis zehn Jahre. Die niedrigste Sterblichkeitsrate konnte alters- und geschlechtsunabhängig bei einem BMI zwischen $22,5 \mathrm{~kg} / \mathrm{m}^{2}$ und $25,0 \mathrm{~kg} / \mathrm{m}^{2}$ beobachtet werden. Davon ausgehend nahm das Mortalitätsrisiko pro Anstieg um fünf BMI-Einheiten um 30\% zu (6). 


\subsubsection{Prävalenz}

Die Adipositas-Prävalenz hat sich weltweit seit 1975 fast verdreifacht. Nach Angaben der WHO hatten im Jahr 2016 mehr als 1,9 Billionen (39\%) Erwachsene weltweit einen BMI über $25 \mathrm{~kg} / \mathrm{m}^{2}$, worunter über 650 Millionen (13\%) als adipös einzustufen waren (BMI $\left.\geq 30 \mathrm{~kg} / \mathrm{m}^{2}\right)$. Frauen (15\%) waren dabei häufiger von Adipositas betroffen als Männer (11\%). Zudem waren 2016 weltweit bereits 41 Millionen Kinder unter fünf Jahren übergewichtig oder adipös. Inzwischen lebt die Mehrheit der Weltbevölkerung in Ländern, in denen mehr Menschen an Übergewicht und Adipositas sterben, als an Untergewicht (7).

In Deutschland sind mittlerweile nahezu ein Viertel aller Erwachsenen von Adipositas betroffen. Die Auswertungen des sog. „Bundes-Gesundheitssurveys (BSG98)“ im Jahr 1998 ergaben eine Adipositas-Prävalenz von 18,9\% unter Männern und 22,5\% unter Frauen (8). Nach den Daten des von 2008 bis 2011 durchgeführten ersten Teils der sog. „Studie zur Gesundheit Erwachsener in Deutschland (DEGS1)“ des Robert-Koch-Instituts stieg die Häufigkeit eines BMI $\geq 30 \mathrm{~kg} / \mathrm{m}^{2}$ seitdem unter Männern auf 23,3\% und unter Frauen auf 23,9\% an. Die Zunahme der Adipositas-Prävalenz war dabei v. a. in der Gruppe der 18 bis 29-Jährigen zu beobachten. Unter den 18 bis 29-jährigen Frauen waren 9,6\% und in der Gruppe der 30 bis 39-jährigen Frauen 17,9\% adipös. Aus der DEGS1-Studie ging zudem hervor, dass die Erkrankungshäufigkeit der Adipositas in Deutschland unter Frauen in den neuen (26,1\%) im Vergleich zu den alten Bundesländern $(23,4 \%)$ höher ist und in allen Altersgruppen mit einem geringeren sozioökonomischen Status zunimmt (9). Der relativ starke Anstieg der Adipositas bei jungen Erwachsenen passt zu der beobachteten hohen Prävalenz von Fettleibigkeit unter Kindern und Jugendlichen. Den Ergebnissen des bundesweiten sog. „Kinder- und Jugendgesundheitssurveys“ zufolge waren im Zeitraum von 2003 bis 2006 bereits 15,0\% der Kinder und Jugendlichen in Deutschland im Alter von drei bis 17 Jahren übergewichtig (BMI >90. Perzentile) und darunter 6,3\% adipös (BMI >97. Perzentile). Unter den 14 bis 17-jährigen Mädchen lag die Adipositas-Prävalenz bei 8,9\%, was fast so hoch ist, wie unter den 18 bis 29-jährigen Frauen der DEGS1-Studie (10). Ziel der Adipositasprävention sollten daher laut Mensink et al. insbesondere Kinder und Jugendliche sowie junge Erwachsene darstellen (9). Wissenschaftler des Instituts für Gesundheits- und Sozialforschung veröffentlichten im Jahr 2016 das sog. „Weißbuch Adipositas“, um die aktuelle Versorgungssituation der Adipositas in Deutschland darzustellen und Anregungen für die künftige Organisation und Optimierung zu geben (11). 
Laut dem sog. „Obesity Update“ der Organisation for Economic Cooperation and Development (OECD) waren im Jahr 2015 19,5\% der erwachsenen Bevölkerung der OECD-Staaten adipös. Dabei war die Adipositas-Prävalenz in Deutschland im internationalen Vergleich im oberen Mittelfeld einzuordnen. Angeführt wurde die Statistik von den USA, gefolgt von Mexiko, Neuseeland, Ungarn, Australien und Kanada. Innerhalb der europäischen Länder stand Großbritannien an erster Stelle des Rankings. Asiatische Länder wie Japan und Korea sowie einige europäische Länder, darunter Norwegen, Schweden, Niederlande und Dänemark, wiesen die niedrigsten Adipositas-Prävalenzen auf (12).

In den USA betrug die Prävalenz von Adipositas laut den Daten der sog. „National Health and Nutrition Examination Survey“ im Jahr 2014 insgesamt 37,9\%. Darunter waren 35,2\% der Männer und 40,5\% der Frauen betroffen (13). In der Gruppe der 20 bis 39-jährigen Frauen belief sich die Krankheitshäufigkeit der Adipositas im Jahr 1999 noch auf 28,4\%, stieg bis 2008 auf 34,0\% an und erreichte 2014 ein Maximum von 37,0\% (13-15). Während die durchschnittliche Lebenserwartung der US-Amerikaner in den letzten 200 Jahren stetig anstieg, erwarten Experten aufgrund der erheblichen Zunahme der Adipositas-Prävalenz ein Ende des Anstiegs oder sogar einen Rückgang innerhalb der ersten Hälfte des 21. Jahrhunderts (16).

Die allgemeine Zunahme der Prävalenz von Adipositas in der Gesamtbevölkerung betrifft auch die Gruppe der Frauen im gebärfähigen Alter, wodurch das Dissertationsthema im Bereich der Geburtshilfe zunehmend an Bedeutung gewinnt.

Die sog. „Bundesauswertung Geburtshilfe“ wurde im Zeitraum von 2005 bis 2015 nacheinander von drei verschiedenen deutschen Instituten für Qualitätssicherung durchgeführt. Die Daten der jährlich veröffentlichten Auswertungen zeigten einen Anstieg der Prävalenz von Adipositas in der Schwangerschaft von 10,8\% im Jahr 2005 auf 13,9\% im Jahr 2015 in Deutschland (1719). In Bayern war laut den Daten der Bayerischen Arbeitsgemeinschaft für Qualitätssicherung in der stationären Versorgung (BAQ) eine Zunahme der Rate adipöser Schwangerer von 10,9\% im Jahr 2005 auf 12,5\% im Jahr 2015 zu verzeichnen (20). In beiden Fällen diente der maternale BMI bei Erstuntersuchung in der Schwangerschaft als Bezugsgröße. Die entsprechenden Kurven der Prävalenz sind in Abbildung 1 dargestellt, wobei deutlich wird, dass die AdipositasPrävalenz in Bayern im Vergleich zur bundesweiten Auswertung einen weniger starken Anstieg aufweist. Als Erklärung dafür kann die höhere Adipositas-Prävalenz unter Frauen in den neuen im Vergleich zu den alten deutschen Bundesländern der DEGS1-Studie angesehen werden (9). 


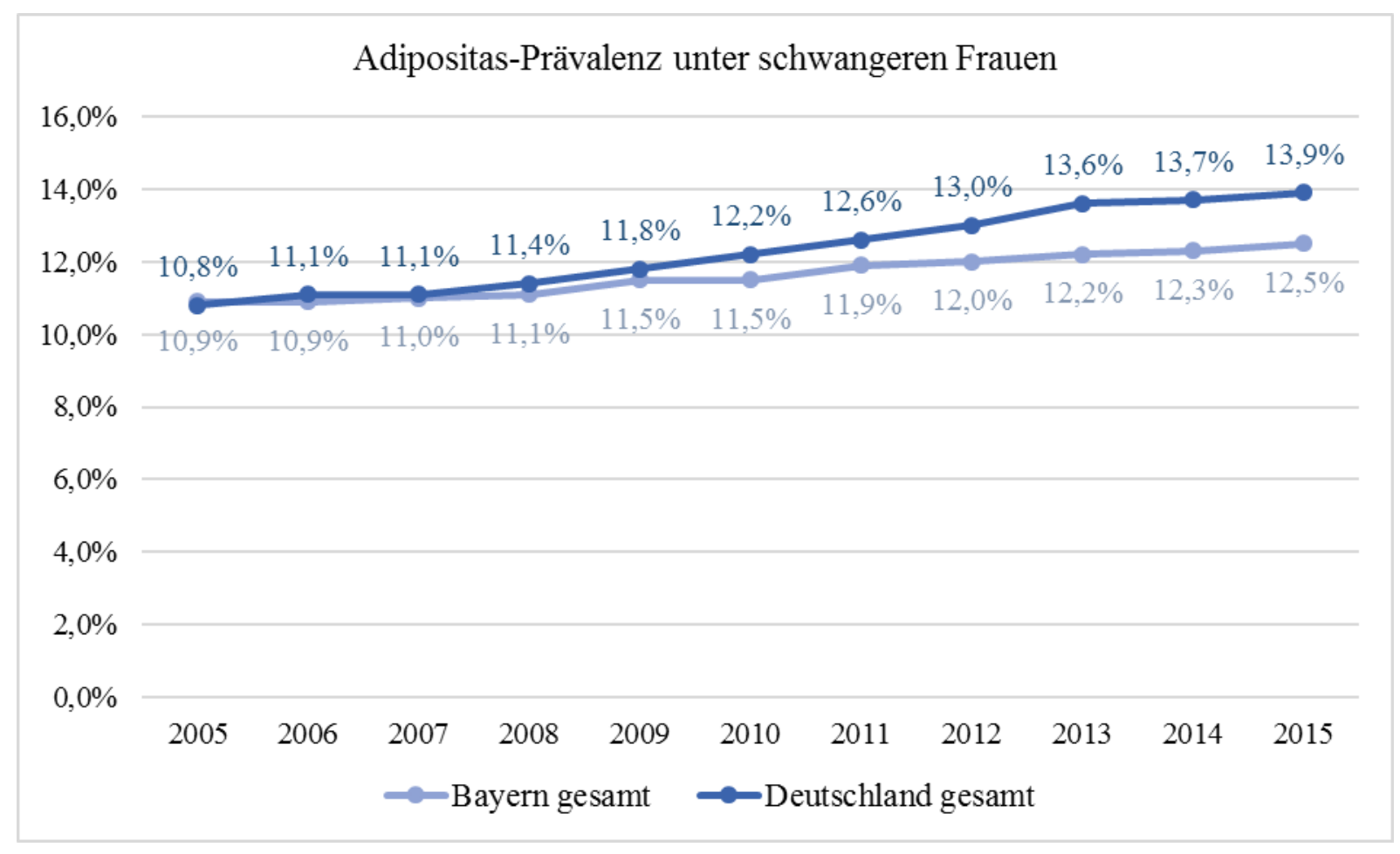

Abb. 1 Relative Häufigkeit von Adipositas in der Schwangerschaft in Bayern und Deutschland von 2005 bis 2015

Nach Angaben des sog. „European Perinatal Health Report“ im Jahr 2010 war die AdipositasPrävalenz unter schwangeren Frauen, gemessen am BMI bei Erstuntersuchung, im europäischen Vergleich in Schottland (20,7\%) am höchsten, während für Frankreich (9,9\%), Slowenien $(9,0 \%)$ und Polen $(7,1 \%)$ die niedrigsten Werte angegeben wurden $(21)$. 


\subsubsection{Auswirkungen auf Schwangerschaft, Geburt, kindliches Outcome und Wochen- bett}

Die Risiken, die mit Adipositas in der Schwangerschaft einhergehen, sind seit langem Gegenstand intensiver Forschung. Eine der ersten Veröffentlichungen geht auf das Jahr 1949 zurück, wobei maternale Adipositas mit einem erhöhten Risiko für Diabetes mellitus in der Schwangerschaft und einem hohen kindlichen Geburtsgewicht in Verbindung gebracht wurde (22). Seither wurden zahlreiche Folgestudien veröffentlicht, die über negative Auswirkungen von maternaler Adipositas auf den Schwangerschafts-, Geburts- und Wochenbettverlauf sowie das kindliche Outcome berichten:

Adipöse Frauen haben ein erhöhtes Risiko für spontane und habituelle Aborte (23-27). Verglichen mit normalgewichtigen Schwangeren treten zudem bei adipösen Frauen folgende Schwangerschaftsrisiken häufiger auf:

- Gestationsdiabetes mellitus $(24,28-38)$

- Gestationshypertonie und Präeklampsie (24, 28, 32, 34, 36-40)

- $\quad$ Nikotinabusus $(28,32,34,36,38)$

- Depressionen und Angststörungen (41)

- Harnwegsinfekte $(24,37,42)$

Als Gestationsdiabetes mellitus (GDM) bezeichnet man eine Glukosetoleranzstörung, die in der Schwangerschaft erstmalig diagnostiziert wurde (43). Neben einer genetischen Disposition und einem mütterlichen Alter über 35 Jahre stellt ein präkonzeptionell erhöhter BMI über 30 $\mathrm{kg} / \mathrm{m}^{2}$ einen der Hauptrisikofaktoren für die Entwicklung eines GDM dar (33).

In der Literatur schwanken die Angaben zur Krankheitshäufigkeit von GDM zwischen 1\% und $4 \%$ in Abhängigkeit von der untersuchten Population und der verwendeten diagnostischen Tests (44). Nach den Angaben der BAQ lag die GDM-Prävalenz in Bayern von 2000 bis 2015 im Mittel bei 2,8\%, wobei eine Zunahme der Frequenz über die Jahre zu beobachten war. Der Anteil von Gestationsdiabetikerinnen stieg ausgehend von 0,8\% im Jahr 2000 auf das Maximum von 4,3\% im Jahr 2015 an, was einer Erhöhung um mehr als das Fünffache entspricht. Die Kurve der GDM-Prävalenz zeigt vergleichsweise einen steileren Verlauf, folgt jedoch dem bereits beschriebenen Anstieg der Adipositas-Prävalenz in Gesamtbayern von 10,0\% auf 12,5\% innerhalb der Jahre 2000 bis 2015, was Abbildung 2 verdeutlicht (20). 


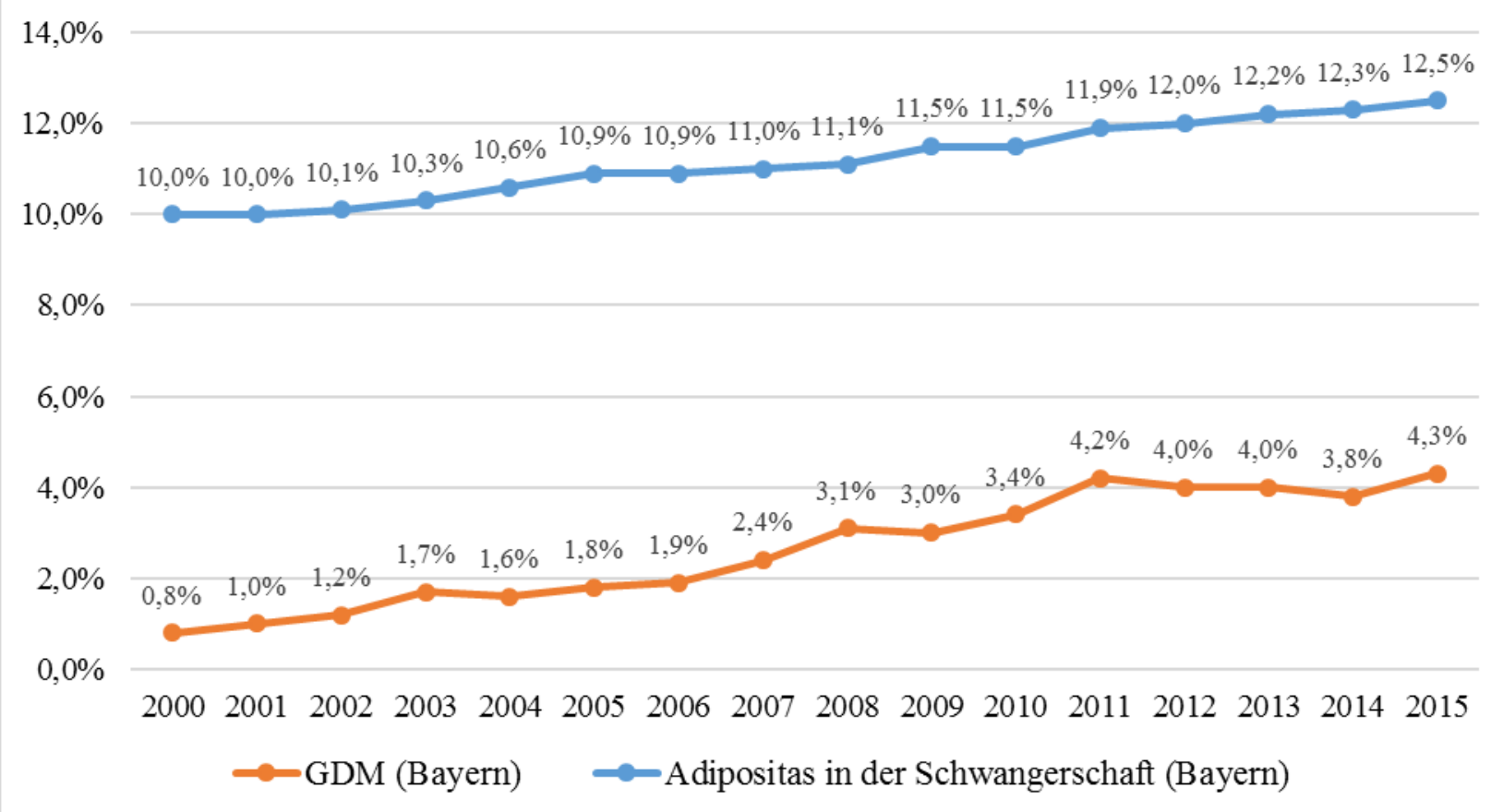

Abb. 2 Relative Häufigkeit von Adipositas in der Schwangerschaft und GDM in Bayern von 2000 bis 2015

Das Vorliegen eines GDM birgt zahlreiche Risiken für Mutter und Kind:

Als unmittelbare Folgen für die Schwangerschaft sind u. a. ein erhöhtes Risiko für Gestationshypertonie und Präeklampsie sowie für Harnwegs- und Candidainfektionen bekannt. Auch ist eine unter Gestationsdiabetikerinnen gesteigerte Rate an Frühgeburten, Geburtseinleitungen, Kaiserschnitt-Entbindungen und Schulterdystokien zu beobachten (30, 31, 44-46). Da wegen der Gefahr eines intrauterinen Fruchttodes (IUFT) bei Schwangeren mit Diabetes mellitus Typ I und II sowie mit insulinpflichtigem GDM eine Überschreitung des errechneten Geburtstermins vermieden werden sollte, wird den Frauen in diesen Fällen entsprechend den Leitlinien eine Geburtseinleitung am errechneten Termin angeboten. Wenn eine adäquate Stoffwechseleinstellung der Schwangeren jedoch nicht möglich ist, besteht die Indikation zur vorzeitigen Entbindung (47).

Die Kinder diabetischer Mütter sind im Vergleich zu denen stoffwechselgesunder Frauen signifikant häufiger von fetaler Makrosomie betroffen, die durch eine überproportionale Zunahme des kindlichen Abdomenumfangs charakterisiert ist. Auch liegen Berichte über ein erhöhtes Risiko für postnatale Komplikationen wie Hypoglykämie, Atemstörungen, Polyglobulie, Hypocalcämie, Hypomagnesiämie und Hyperbilirubinämie für die Neugeborenen von Müttern mit GDM vor (31, 44-46, 48). Der Zusammenhang zwischen mütterlichem Diabetes mellitus und einem hohen kindlichen Geburtsgewicht wurde im Jahr 1952 durch den dänischen Arzt Jørgen 
Pedersen beschrieben. Nach seiner Hypothese führt mütterliche Hyperglykämie während der Schwangerschaft zu fetaler Hyperglykämie und Hyperinsulinämie, was ein verstärktes fetales Wachstum sowie eine Zunahme des fetalen Fettgewebes zur Folge hat (49).

Eine Gestationshypertonie liegt vor, wenn bei einer zuvor normotensiven Schwangeren nach der abgeschlossenen 20. Schwangerschaftswoche (SSW) Blutdruckwerte $\geq 140 / 90 \mathrm{mmHg}$ auftreten. Wird zusätzlich eine Proteinurie nachgewiesen ( $\geq 300$ mg/24 Stunden im Sammelurin), handelt es sich definitionsgemäß um eine Präeklampsie. Zu den besonders schweren Manifestationen der Präeklampsie zählen die Eklampsie, die durch das Auftreten tonisch-klonischer Krampfanfälle gekennzeichnet ist und das HELLP-Syndrom, das durch die Trias Hämolyse (Hemolysis), pathologisch erhöhte Leberenzyme (Elevated Liver enzymes) und Thrombozytopenie (Low Platelets) charakterisiert ist (50). In $15 \%$ bis $25 \%$ der Fälle entwickelt sich aus einer Gestationshypertonie im Verlauf der Schwangerschaft eine Präeklampsie (51).

Neben maternaler Adipositas zählen u. a. Diabetes mellitus, Erstparität, Zustand nach (Z. n.) Präeklampsie, chronische Hypertonie, ein Alter über 40 Jahre und das Vorliegen eines Antiphospholipid-Syndroms zu den Risikofaktoren für die Entwicklung einer Präeklampsie (52, 53).

Aufgrund einer gestörten Perfusion des uteroplazentaren Stromgebietes kann das Vorliegen einer Präeklampsie eine intrauterine Wachstumsrestriktion des Fetus (IUGR), einen intrauterinen Fruchttod sowie Plazentainfarkte und eine vorzeitige Plazentalösung zur Folge haben. Da die Entbindung die einzige kausale Therapie darstellt, ist die Rate der Frühgeburten bei präeklamptischen Frauen erhöht (54). Nach Angaben der WHO stellen hypertensive Schwangerschaftserkrankungen weltweit in den Industrieländern mit 16,1\% die häufigste Ursache mütterlicher Todesfälle dar, gefolgt von Thromboembolien und Hämorrhagien mit 14,9\% bzw. 13,4\% (55). Nach Auftreten einer hypertensiven Schwangerschaftserkrankung ist für die betroffenen Frauen mit einem lebenslang signifikant erhöhten relativen Risiko $(R R)$ für Hypertonie ( $R R=3,7)$, ischämische Herzerkrankung $(R R=2,2)$, zerebralen Insult $(R R=1,8)$ und für die kardiovaskulär bedingte Mortalität $(\mathrm{RR}=1,5)$ zu rechnen (56). 
Laut den Ergebnissen zahlreicher Autoren besteht ein positiver Zusammenhang zwischen Adipositas der Mutter und den folgenden Ereignissen hinsichtlich des Geburtsverlaufs:

- Entbindung per primärer und sekundärer Sectio caesarea $(24,25,29,32,34,36,37,42$, $57-68)$

- Entbindung per Not-Sectio caesarea $(42,67,69,70)$

- Frühgeburt $(28,29,32,36,70-75)$ sowie zeitliche Übertragung der Schwangerschaft $(37,67,70,74)$

- Geburtseinleitung $(24,25,29,37,42,67,68,76)$

- Einsatz eines Wehenmittels unter der Geburt $(62,68,70)$

- Schulterdystokie $(24,36,42,64)$ und kindliche Verletzungen infolge einer Schulterdystokie $(36,77)$

Die Schulterdystokie, die als Geburtsstillstand infolge des Ausbleibens der Rotation der Schultern beim Durchtritt durch den Geburtskanal definiert ist, stellt eine geburtshilfliche Notfallsituation dar $(78,79)$. In der Literatur schwanken die Angaben zur Inzidenz der Schulterdystokie zwischen 0,9\% und 3,6\% (24, 34, 36, 80). Komplikationen, die aus einer erschwerten Schulterentwicklung resultieren können, sind fetale Asphyxie, Schädigungen des Plexus brachialis, Frakturen der Klavikula und des Humerus sowie mütterliche Weichteilverletzungen. Die Adipositas der Mutter konnte als einer der wichtigsten Risikofaktoren für eine Schulterdystokie identifiziert werden. Weitere Faktoren, die mit einer erhöhten Wahrscheinlichkeit für das Auftreten einer Schulterdystokie einhergehen, sind u.a. Makrosomie des Kindes, mütterlicher Diabetes mellitus, Multiparität, vaginal-operativer Entbindungsmodus und eine protrahierte Austreibungsphase der Geburt $(78,79)$.

Während der Entbindung werden bei adipösen Frauen im Vergleich zu Normalgewichtigen zudem folgende Outcomes beobachtet:

- Häufiger mekoniumhaltiges Fruchtwasser $(25,62,70,81,82)$

- Verlängerte Eröffnungsperiode (EP) sowie verkürzte Austreibungsperiode (AP) der Geburt $(25,42,67,68,76)$

- Häufiger peripartale Blutungen $(24,37,42,70,76,83)$ 
Mit Blick auf das Outcome der Kinder adipöser Frauen im Vergleich zu dem der Kinder normalgewichtiger Frauen werden folgende Zusammenhänge deutlich:

- Höheres Geburtsgewicht (24, 29, 32, 34, 36, 68, 70, 84), häufigere Einstufung als Largefor-Gestational-Age (LGA) $(24,37,60,85)$ sowie seltenere Einstufung als Small-forGestational-Age (SGA) $(24,28,37)$

- Niedrigere Apgar-Werte nach der ersten, fünften und zehnten Lebensminute (29, 34, $37,64,86,87)$

- Häufigere Azidosen $(64,71)$

- Erhöhte Rate kongenitaler Anomalien (32, 88-96)

- Häufigere Verlegung auf eine neonatologische Station $(29,32,36,37,42,70)$

- Erhöhtes Risiko für einen IUFT $(26,62,64,97-99)$ sowie einen neonatalen Tod innerhalb der ersten sieben Lebenstage $(26,36,74,97)$

Bezogen auf den Wochenbettverlauf ist eine Abnahme der Stillrate mit zunehmender mütterlicher BMI-Kategorie zu beobachten (37, 100-104). Auch das Risiko für eine Wundinfektion im Wochenbett $(37,70,71,81,105,106)$ sowie für postpartales Fieber $(25,37)$ ist laut der aktuellen Studienlage für adipöse Frauen im Vergleich zu normalgewichtigen Wöchnerinnen erhöht. Adipöse Mütter scheinen dagegen ein geringeres Risiko für eine Anämie im Wochenbett zu haben, im Vergleich zu normalgewichtige Frauen $(37,106)$.

Das Stillen ist sowohl für die Mutter als auch für das Neugeborene mit positiven gesundheitlichen Effekten assoziiert und fördert die emotionale Bindung zwischen Mutter und Kind. Die Ergebnisse verschiedener Studien deuten darauf hin, dass Stillen das Risiko für die Entwicklung eines mütterlichen Diabetes mellitus Typ II oder eines metabolischen Syndroms im späteren Leben reduziert und zwar umso deutlicher, je länger gestillt wurde (107-110). Die Wahrscheinlichkeit für einen mütterlichen Diabetes mellitus Typ II sank unter Pluriparae pro Jahr Stillen um 14\% bis 15\% $(109,110)$. Stillen erhöht den mütterlichen Energiebedarf um ca. 500 Kilokalorien am Tag, was stillenden Frauen das Abnehmen nach der Geburt erleichtert. Nach Krause et al. wogen die voll-stillenden Mütter und die zum Teil stillenden Mütter sechs Monate nach der Geburt im Mittel 1,38 kg bzw. 0,84 kg weniger, als Frauen, die ihre Kinder mit der Flasche aufzogen (111). Auch konnte in verschiedenen Studien ein schützender Effekt des Stillens bzgl. der Inzidenz von Brust (112)- und Ovarialkarzinomen (113) beobachtet werden. 
Die sog. „Studie zur Gesundheit von Kindern und Jugendlichen in Deutschland“ untersuchte den Zusammenhang zwischen dem mütterlichen Stillverhalten und der Prävalenz von Übergewicht und Adipositas unter den Nachkommen. Kinder, die mindestens vier Monate gestillt wurden, hatten ein signifikant reduziertes Risiko, im Alter von sieben bis zehn Jahren übergewichtig $(\mathrm{OR}=0,67)$ oder adipös $(\mathrm{OR}=0,56) \mathrm{zu}$ sein im Vergleich zu Altersgenossen, die nicht oder für einen kürzeren Zeitraum gestillt wurden (114). Nach Schäfer-Graf et al. war die Wahrscheinlichkeit, im Alter von zwei bis acht Jahren übergewichtig zu sein (BMI >90. Perzentile), für die Nachkommen von Müttern mit GDM um 45\% reduziert, wenn die Stilldauer mindestens drei Monate betrug (104). Zudem wurde über einen protektiven Effekt der Muttermilch u. a. hinsichtlich kindlicher Infektionskrankheiten (115), der neonatalen Mortalität zwischen 28 Tagen und einem Jahr (116) sowie der Inzidenz von Asthma in der Kindheit (117) berichtet. Weiterhin scheint das Stillen einen positiven Einfluss auf die kognitive Entwicklung der Kinder zu nehmen (118).

Im Jahr 2016 wurde ein groß angelegter systematischer Review zum Thema Stillhäufigkeit und Stilldauer in Deutschland veröffentlicht, der 35 Studien im Zeitraum von 1990 bis 2012 beinhaltet. Die Auswertungen ergaben, dass zwischen 72\% und 97\% der Mütter ihre Säuglinge nach der Geburt stillten. Innerhalb der ersten zwei Monate war der stärkste Abfall der Stillquote zu verzeichnen, sodass im Alter von sechs Monaten nur noch etwa 50\% der im Analysezeitraum geborenen Kinder in Deutschland gestillt wurden (119).

\subsubsection{Langzeitwirkungen durch Perinatale Programmierung}

Neben den in Kapitel 1.1.3 beschriebenen unmittelbaren Auswirkungen von maternaler Adipositas auf Schwangerschaft, Geburt, kindliches Outcome und Wochenbett, liegen auch Berichte über Langzeitwirkungen für die Nachkommen adipöser Frauen vor.

Bereits 1997 demonstrierte eine finnische Studie einen positiven Zusammenhang zwischen einem hohen mütterlichen BMI und der Mortalitätsrate durch kardiovaskuläre Krankheiten in der Folgegeneration (120). Fall et al. berichteten 1998 über eine höhere Inzidenz von Diabetes mellitus Typ II unter den Nachkommen von Frauen, die in der Schwangerschaft ein überdurchschnittlich hohes Gewicht hatten (121). Auch in Folgestudien wurde eine Erhöhung der kindlichen Erkrankungsrate an Adipositas sowie an kardiovaskulären und metabolischen Gesundheitsproblemen im späteren Leben als Konsequenz von maternaler Adipositas in der Schwangerschaft beobachtet. Zudem scheinen auch respiratorische und kognitive Störungen in der 
Kindheit laut der aktuellen Studienlage mit mütterlicher Fettleibigkeit in Zusammenhang zu stehen (122-128). Eine Metaanalyse, die 45 Studien im Zeitraum von 1970 bis 2012 einschloss, berichtete über ein 3-fach erhöhtes Risiko für die Entwicklung von Adipositas in der Kindheit in der Folgegeneration von Müttern mit einem BMI $\geq 30 \mathrm{~kg} / \mathrm{m}^{2}$, im Vergleich zu den Nachkommen normalgewichtiger Schwangerer (129).

Adipositas in der Schwangerschaft geht, auch bei ansonsten gesunden Frauen, mit Hyperinsulinämie, Hypertonie, Dyslipidämie, endothelialer Dysfunktion und chronischer Inflammation einher, was Ramsay et al. in ihrer Studie erstmals demonstrierten. Im Vergleich zu normalgewichtigen Schwangeren waren die Leptin- und Nüchtern-Insulinwerte bei den adipösen Studienteilnehmerinnen mehr als verdoppelt und die Konzentration der Entzündungsparameter Interleukin-6 und C-reaktives Protein sowie die Nüchtern-Triglycerid-Werte wesentlich erhöht. Zudem zeigte sich sowohl die endothel-abhängige als auch die endothel-unabhängige vasodilatatorische Reaktion in der Gruppe der fettleibigen Frauen als signifikant reduziert (130). Dieses Spektrum an Risikofaktoren kann laut den Autoren nicht nur zur Entwicklung von Schwangerschaftskomplikationen wie GDM oder Präeklampsie beitragen, sondern auch durch die sog. Perinatale Programmierung das Risiko des sich entwickelnden Fetus für chronische Erkrankungen im späteren Leben erhöhen (130-132).

Der Begriff der Perinatalen Programmierung beschreibt den Prozess, bei dem während kritischer prä- und neonataler Entwicklungsphasen durch die Einwirkung von Faktoren wie Ernährung oder Hormonen die künftige Funktionsweise von Organen bzw. Organsystemen dauerhaft geprägt wird (133-135). Die zugrundeliegenden Mechanismen sind noch nicht vollständig geklärt, wobei epigenetischen Einflüssen auf die Genexpression eine Schlüsselrolle zugeschrieben wird $(128,136)$.

Nach der Theorie von Plagemann resultiert eine kindliche Adaptation an unphysiologische Umwelteinflüsse wie mütterliche Adipositas, Diabetes mellitus oder neonatale Überernährung in einem fetalen bzw. perinatalen Hyperinsulinismus und Hyperleptinismus. Dieser Zustand kann eine neuroendokrine Fehlprogrammierung im Sinne einer hypothalamischen Insulin- und Leptinresistenz zur Folge haben. Dabei wirken Insulin und Leptin physiologischerweise als periphere Sättigungssignale. Auf der Basis der Fehleinstellung von Kontrollsystemen endokriner Funktionskreise können sich durch eine gestörte Regulation von Nahrungsaufnahme, Körpergewicht und Stoffwechsel im späteren Leben chronische Erkrankungen wie Adipositas und Diabetes mellitus sowie deren Folgeerkrankungen entwickeln (Abb. 3) (133-135, 137). 


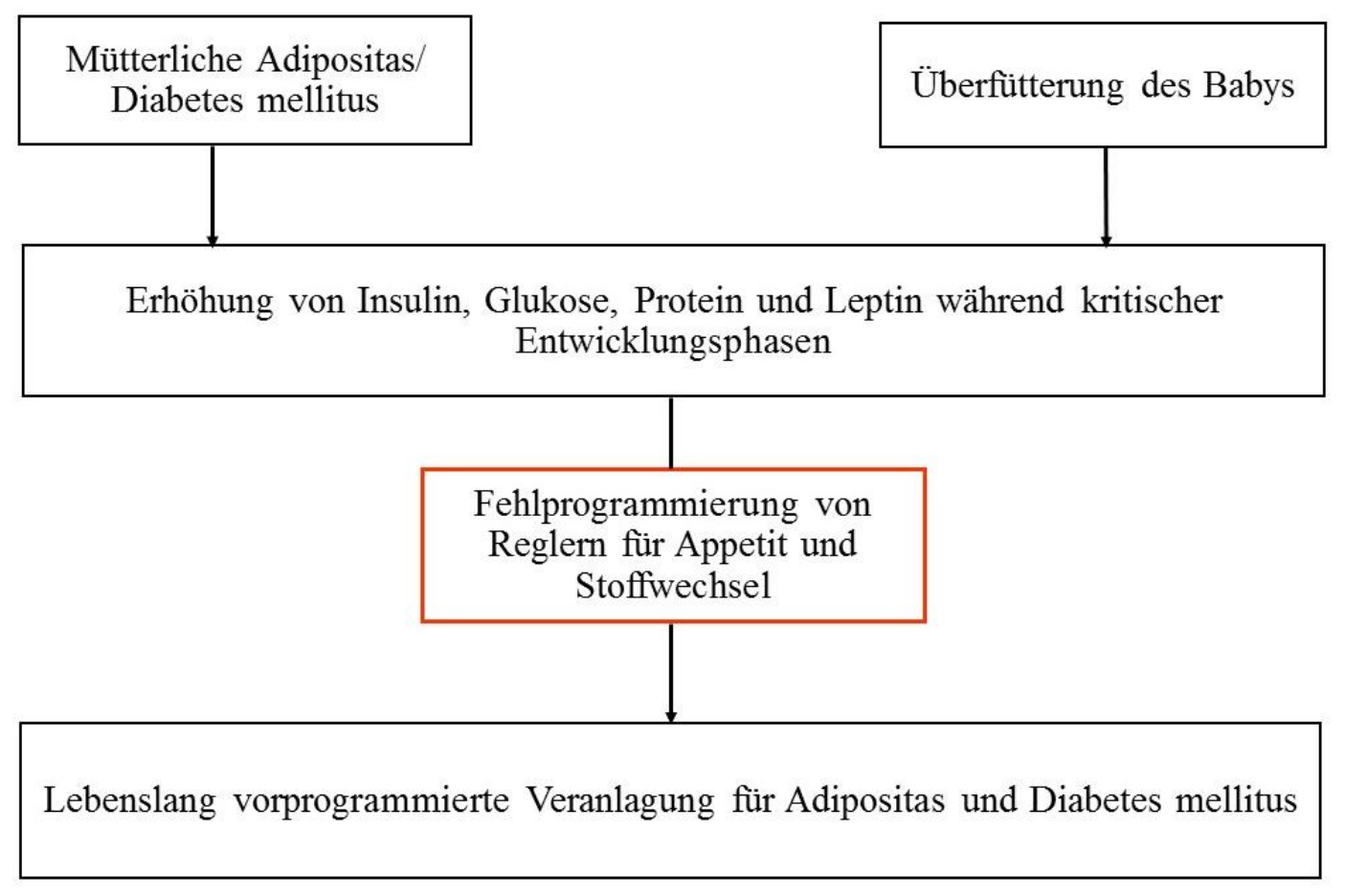

Abb. 3 Allgemeines Konzept zur Perinatalen Programmierung eines erhöhten Adipositas- und Diabetesrisikos nach Schellong 2008 (135)

Eine neonatale Überernährung kann u. a. durch die Förderung des Stillens vermieden werden, da der im Vergleich zu Muttermilch erhöhte Proteinanteil der Flaschennahrung in einer verstärkten Insulinantwort des Neugeborenen resultiert (138).

Neben dem Einfluss der Perinatalen Programmierung werden auch genetische und umweltbedingte Faktoren sowie die Art des Lebensstils als mögliche Ursachen von kindlicher Adipositas im späteren Leben infolge von mütterlicher Adipositas in der Schwangerschaft in Betracht gezogen (126-128). 


\subsection{Zielsetzung und Fragestellungen}

Ziel der Arbeit war es, ausgewählte, in der Literatur beschriebene Auswirkungen von Adipositas in der Schwangerschaft anhand der Patientendaten in der Klinik St. Hedwig zu analysieren und zu vergleichen. Der Vergleich wurde zwischen schwangeren Frauen mit Normalgewicht, Übergewicht und Adipositas im Zeitraum von 2000 bis 2015 vorgenommen. Der Fokus der Analyse lag dabei auf dem Schwangerschafts-, Geburts- und Wochenbettverlauf sowie dem kindlichen Outcome.

Um eine Aussage über die Prävalenz von Übergewicht und Adipositas in der Schwangerschaft in der Klinik St. Hedwig treffen zu können, war diese zum einen gesamt und zum anderen pro Jahr darzustellen. Tendenzen der Zu- oder Abnahme galt es zu prüfen. Das Patientenkollektiv war auf etwaige Unterschiede zwischen den definierten BMI-Gruppen bzgl. der Ausprägung ausgewählter Variablen zu untersuchen, welche die maternalen Charakteristika, den Schwangerschafts-, Geburts- und Wochenbettverlauf sowie das kindliche Outcome skizzieren. Die einzelnen herangezogenen Variablen sind in Kapitel 2.3 beschrieben.

Das retrospektive Forschungsvorhaben wurde von der Ethikkommission der Universität Regensburg geprüft und in deren Sitzung am 09.12.2015 wurden keine Einwände gegen die Durchführung erhoben (15-104-0331). 


\section{PATIENTENKOLLEKTIV UND METHODIK}

\subsection{Datenerfassung und -aufbereitung}

Als Grundlage der vorliegenden retrospektiven Studie dienten die Daten der Geburten in der Klinik für Frauenheilkunde und Geburtshilfe St. Hedwig in Regensburg im Zeitraum von 2000 bis 2015. Diese Angaben werden von den betreuenden Ärzten in die klinikinterne KreißsaalDatenbank eingegeben und gespeichert. Die Daten der zu untersuchenden Variablen wurden mithilfe des Programms Viewpoint, Version 5.6.25.282, GE Medical, aus der Kreißsaal-Datenbank in das Programm Microsoft Office Excel 2007 extrahiert, aufbereitet und codiert. Bei fehlender Übertragung einzelner Daten in die Kreißsaal-Datenbank oder bei Dokumentation unplausibler Werte wurden diese - soweit möglich - durch Einsicht von Patientenakten nachgetragen bzw. korrigiert.

Basierend auf der Zielsetzung der Arbeit - der Vergleich von Frauen verschiedener BMI-Kategorien - wurden die Patientinnen anhand ihres BMI laut Mutterpass gemäß den Vorgaben der WHO in Gruppen eingeteilt (siehe Tab. 1). Die BMI-Kategorie Normalgewicht diente dabei als Referenzgruppe.

\subsection{Auswahlkriterien und Beschreibung des Studienkollektivs}

Zu den Ausschlusskriterien zählten lediglich das Vorliegen einer Mehrlingsschwangerschaft, der BMI kleiner $18,5 \mathrm{~kg} / \mathrm{m}^{2}$ sowie die fehlende Dokumentation der mütterlichen Daten $\mathrm{zu}$ Größe und Gewicht zur Berechnung des BMI vor der Schwangerschaft. Das untersuchte Studienkollektiv umfasste somit ein breites Abbild der Eigenschaften der Schwangeren.

Von 26.06.2000 bis 31.12.2015 wurden in der Klinik St. Hedwig insgesamt 29180 Einlinge geboren, deren Mütter einer BMI-Kategorie zugeordnet werden konnten. Davon waren 1426 Frauen (4,9\%) untergewichtig, 18809 Patientinnen (64,5\%) normalgewichtig, 5741 Frauen $(19,7 \%)$ übergewichtig und 3204 Schwangere $(11,0 \%)$ zählten zu der BMI-Kategorie Adipositas. Innerhalb der Gruppe der adipösen Frauen konnten 2009 Schwangere (6,9\%) der BMIKategorie Adipositas Grad I, 774 Frauen (2,7\%) der Kategorie Adipositas Grad II und 421 Frauen (1,4\%) der Kategorie Adipositas Grad III zugeordnet werden (Abb. 4). 


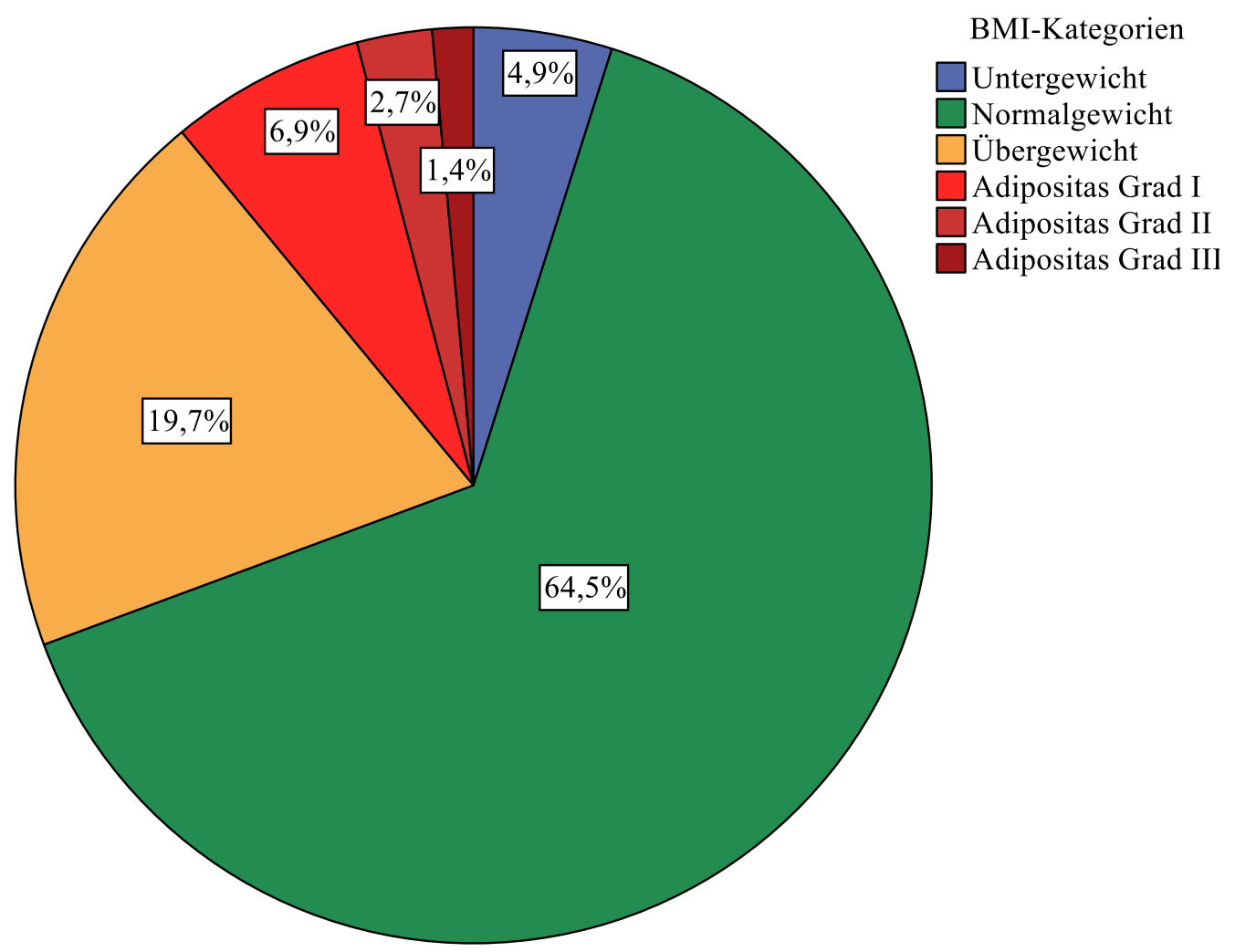

Abb. 4 Relative Häufigkeitsverteilung der verschiedenen maternalen BMI-Kategorien im Studienkollektiv $(N=29180)$

Nach Ausschluss der 1426 untergewichtigen Frauen umfasste das Studienkollektiv 27754 Geburten, die im Rahmen dieser Arbeit auf etwaige Unterschiede in der Ausprägung ausgewählter Variablen (Kapitel 2.3) getestet wurden.

\subsection{Variablen und Definitionen}

Zur Beantwortung der Fragestellungen wurden die Daten folgender Variablen ausgewertet:

\section{Maternale Daten:}

- Größe, Gewicht, BMI vor der Schwangerschaft nach Angabe im Mutterpass

- Alter der Mutter bei Entbindung

- Parität

- Aborte in früheren Schwangerschaften

- Präexistenter Diabetes mellitus

- Chronische Hypertonie

- Gewichtszunahme während der Schwangerschaft

- Aufenthaltsdauer der Mutter in der Klinik insgesamt 


\section{Befundete Schwangerschaftsrisiken:}

- Gestationsdiabetes mellitus

- Gestationshypertonie

- Präeklampsie, HELLP-Syndrom, Eklampsie

- Nikotinabusus

- Besondere psychische Belastung

- Harnwegsinfekt

- Fetale Wachstumsrestriktion

- Vorzeitige Wehen (Zervixwirksam vor der vollendeten 37. SSW)

\section{Geburtsverlauf:}

- Vorzeitiger Blasensprung

- Geburtsmodus und Indikationen zur Sectio caesarea

- Gestationsalter bei Geburt

- Geburtseinleitung und Indikationen

- Wehenmittel (Oxytocin-Tropf)

- Schulterdystokie und resultierende kindliche Geburtsverletzungen

- Grünes Fruchtwasser

- Geburtsdauer insgesamt, protrahierter Verlauf oder Geburtsstillstand in EP/AP

- Blutverlust der Mutter unter der Geburt (vom Geburtshelfer abgeschätzt)

\section{Kindliche Daten:}

- Geburtsgewicht und Geburtsgewichtsperzentile

- Apgar-Werte nach einer, fünf und zehn Minuten postnatal

- PH- und Base Excess (BE)-Wert im Nabelschnurblut

- Fehlbildungen, Vorliegen einer pränatalen Diagnose

- Verlegungsrate und Verlegungsgründe

- Totgeburt: Intrauteriner Fruchttod und subpartualer Tod

- Neonataler Tod innerhalb der ersten sieben Lebenstage

\section{Wochenbettverlauf:}

- Stillen

- Revisionsbedürftige Wundheilungsstörungen/ Wundinfektion nach Sectio caesarea

- Fieber post partum: Körpertemperatur über $38^{\circ} \mathrm{C}$ länger als zwei Tage

- Anämie: Hämoglobinwert $<10 \mathrm{~g} / \mathrm{dl}$ 
Bzgl. der Definition einiger Variablen herrschen internationale Unterschiede. In dieser Arbeit wurden folgende, in Deutschland geltende oder gebräuchliche Definitionen angewendet:

Die Totgeburt ist gemäß $§ 31$ II in Verbindung mit $§ 31$ I Personenstandsverordnung (PStV) definiert als ein Fetus, der nach der Geburt kein Lebenszeichen wie Herzschlag, Pulsation der Nabelschnur oder Spontanatmung aufweist und dessen Geburtsgewicht mindestens $500 \mathrm{~g}$ beträgt. Bei einem Geburtsgewicht von weniger als $500 \mathrm{~g}$ wird der totgeborene Fetus gemäß $§ 31$ III PStV als Fehlgeburt bezeichnet.

Die peripartale Hämorrhagie (PPH) ist nach Angaben der aktuellen Leitlinie als ein Blutverlust von mehr als $500 \mathrm{ml}$ nach vaginaler Geburt oder mehr als $1000 \mathrm{ml}$ nach Sectio caesarea zu definieren (139).

Nach den von Voigt et al. berechneten Gewichtsperzentilen, die das kindliche Geburtsgewicht in Abhängigkeit des Geschlechts und des Gestationsalters betrachten, werden Neugeborene mit einem Geburtsgewicht unterhalb der 10. Perzentile als Small-for-Gestational-Age bezeichnet und solche mit einem Geburtsgewicht oberhalb der 90. Perzentile als Large-for-GestationalAge (140).

Nach den Richtlinien des Institute of Medicine (IOM) wird, gemessen am präkonzeptionellen BMI, normalgewichtigen Frauen eine Gewichtszunahme von 11,5 bis 16 kg in der Schwangerschaft empfohlen, übergewichtigen Frauen von 7 bis 11,5 kg und adipösen Frauen von 5 bis 9 $\mathrm{kg}(141)$.

Der Apgar-Score dient der standardisierten Beurteilung des klinischen Zustands von Neugeborenen anhand folgender Kriterien: Aussehen (Hautfarbe), Puls (Herzfrequenz), Grimassen (Reflexe), Aktivität (Muskeltonus) und Respiration (Atmung) (142). In dieser Studie wurden Neugeborene mit Apgar-Werten von 8 bis 10 Punkten als lebensfrisch bezeichnet, während 4 bis 7 Punkte einer mittelgradigen und 0 bis 3 Punkte einer schwergradigen Depression der kindlichen Vitalität entsprachen.

Der kindliche pH-Wert im Nabelarterienblut wurde wie folgt klassifiziert und bewertet:

- >7,30: Normaler $\mathrm{pH}-$ Wert

- 7,20-7,29: Leichte Azidose

- 7,10-7,19: Mittelgradige Azidose

- 7,00-7,09: Fortgeschrittene Azidose

- <7,00: Schwere Azidose 


\subsection{Statistische Auswertung}

Für die statistische Datenanalyse wurde das Programm IBM SPSS Statistics 23 verwendet.

Um den Zusammenhang zwischen zwei kategorialen Variablen zu testen, wurden Kreuztabellen erstellt, die absolute und relative Häufigkeiten der Merkmalsausprägungen zeigen. Zur Überprüfung der statistischen Signifikanz von beobachteten Unterschieden zwischen zwei BMI-Gruppen wurde der Chi-Quadrat-Test angewendet, bei erwarteten Häufigkeitswerten kleiner fünf der exakte Test nach Fisher. Balkendiagramme dienten der Veranschaulichung der ermittelten Häufigkeitsverteilungen.

Die Kennzahlen kontinuierlicher Variablen wie Mittelwert, Standardabweichung (SD), Minimum und Maximum wurden mithilfe der explorativen Datenanalyse berechnet. Nach der Überprüfung auf Normalverteilung und Varianzgleichheit der zu untersuchenden Variablen wurde die einfaktorielle Varianzanalyse (ANOVA) für den Vergleich der Mittelwerte mehrerer Gruppen herangezogen. Anhand von anschließenden paarweisen Mehrfachvergleichen (Post-hoc Tests) konnten statistisch signifikante Unterschiede zwischen den einzelnen Gruppen ermittelt werden. Dabei wurde die Methode der geringsten signifikanten Differenz (LSD) verwendet. Die Verteilung stetiger Variablen wurde grafisch in Form von Boxplot-Diagrammen dargestellt.

Das Signifikanzniveau war für alle Tests auf 5\% festgelegt. In Übereinstimmung mit den Angaben des Programms SPSS wurde auf die Nennung der vierten Kommastelle verzichtet und pWerte kleiner 0,001 als $\mathrm{p}<0,001$ angegeben.

Da in der hier vorliegenden Studie vergleichsweise wenige Ausschlusskriterien festgelegt wurden, konnte ein umfassendes Abbild der Entbindungsdaten in der Klinik St. Hedwig von 2000 bis 2015 dargestellt werden. Es ist darauf hinzuweisen, dass dadurch der beobachtete Zusammenhang zwischen dem BMI der Frau und einer untersuchten Variablen möglicherweise durch eine andere Variable als Störgröße verzerrt wurde. Bei der statistischen Datenanalyse wurde dieser Einfluss an ausgewählten Stellen durch Stratifizierung kontrolliert, d. h. es wurden Subgruppen bzgl. der Ausprägung möglicher Störvariablen gebildet und analysiert. 


\section{ERGEBNISSE}

\subsection{Adipositas-Prävalenz}

Im Untersuchungszeitraum lag die Prävalenz von Übergewicht in der Klinik St. Hedwig insgesamt bei 19,7\%, während 11,0\% der Schwangeren der BMI-Kategorie Adipositas angehörten. Darunter betrug die Prävalenz von Adipositas Grad I 6,9\%, die von Adipositas Grad II 2,7\% und die von Adipositas Grad III 1,4\%. 64,5\% der Schwangeren waren normalgewichtig und 4,9\% der Frauen zählten zur BMI-Gruppe Untergewicht (siehe Abb. 4).

Die Prävalenz von Übergewicht und Adipositas in der Schwangerschaft nahm in der Klinik St. Hedwig von 2000 bis 2015 zu. Der Anteil adipöser Schwangerer schwankte im Untersuchungszeitraum zwischen 9,1\% im Jahr 2004 und dem Maximum von 13,1\% im Jahr 2015. Die Prävalenz von Übergewicht erreichte den niedrigsten Wert 2002 mit 16,4\% und den höchsten Wert 2015 mit 21,3\%. Die höchste Prävalenz von Schwangeren der Kategorie Adipositas Grad III konnte in den Jahren 2011 und 2015 beobachtet werden mit jeweils 2,0\%, der niedrigste Wert im Jahr 2006 mit 1,0\%. Die Prävalenz der verschiedenen BMI-Kategorien pro Jahr von 2000 bis 2015 ist in Abbildung 5 bzw. Tabelle 3 dargestellt.

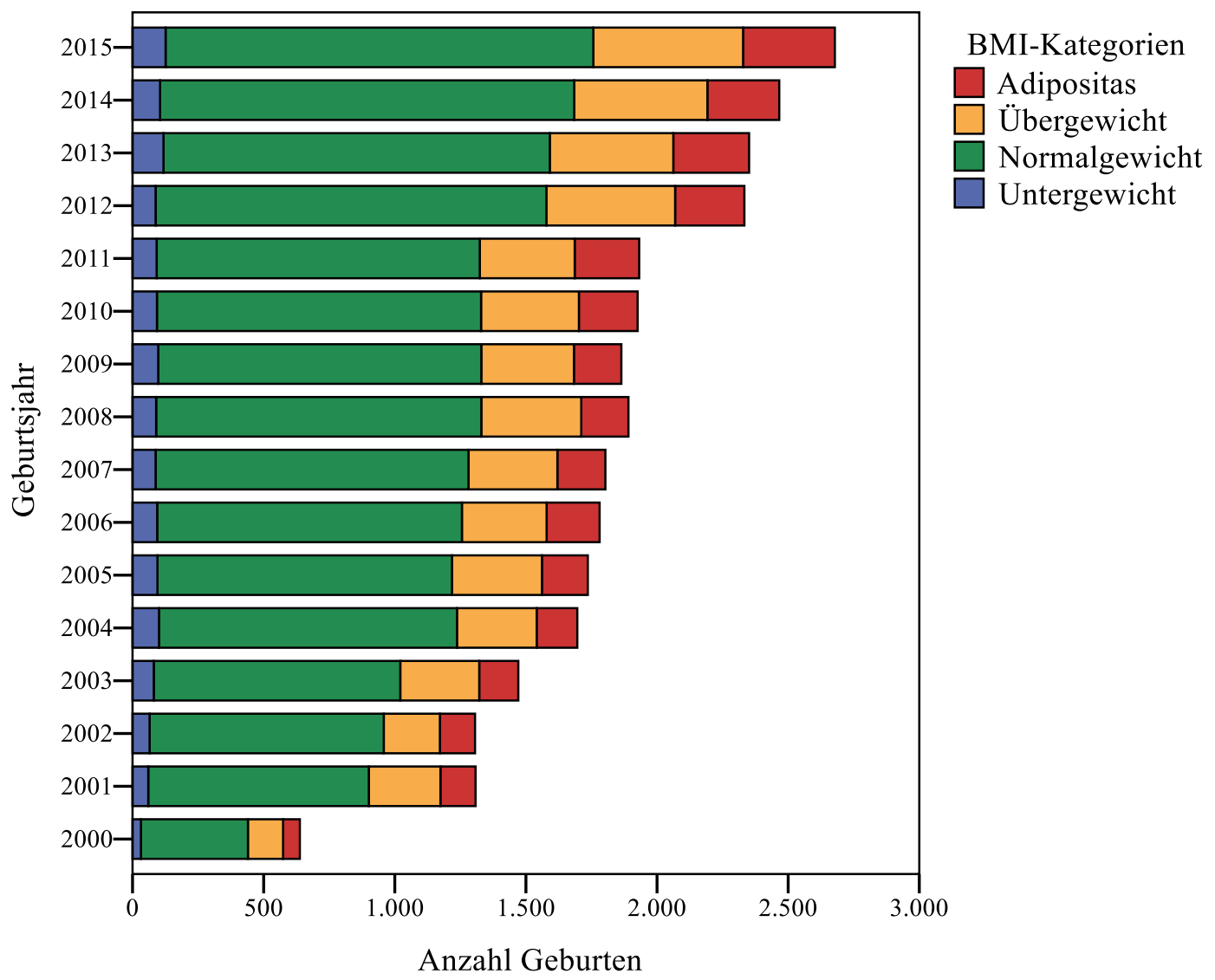

Abb. 5 Absolute Häufigkeiten der verschiedenen maternalen BMI-Kategorien pro Jahr von 2000-2015 (N=29180) 
Tab. 3 Absolute und relative Häufigkeiten der maternalen BMI-Kategorien pro Jahr von 2000 bis 2015

BMI-Kategorien [ $\left.\mathrm{kg} / \mathrm{m}^{2}\right]$

\begin{tabular}{|c|c|c|c|c|c|c|c|c|}
\hline \multirow[b]{2}{*}{ Geburtsjahr } & & & & & & & \multirow[b]{2}{*}{$\begin{array}{c}\geq \mathbf{3 0} \\
\mathrm{n}=3204\end{array}$} & \multirow[b]{2}{*}{$\begin{array}{c}\text { Gesamt } \\
\mathrm{N}=29180\end{array}$} \\
\hline & $\begin{array}{c}<\mathbf{1 8 , 5} \\
\mathrm{n}=1426 \\
\end{array}$ & $\begin{array}{c}\mathbf{1 8 , 5}-\mathbf{2 4 , 9} \\
\mathrm{n}=18809 \\
\end{array}$ & $\begin{array}{c}\mathbf{2 5 , 0 - 2 9 , 9} \\
\mathrm{n}=5741 \\
\end{array}$ & $\begin{array}{c}\mathbf{3 0 , 0 - 3 4 , 9} \\
\mathrm{n}=2009 \\
\end{array}$ & $\begin{array}{c}\mathbf{3 5 , 0 - 3 9 , 9} \\
\mathrm{n}=774 \\
\end{array}$ & $\begin{array}{c}\geq \mathbf{4 0} \\
\mathrm{n}=421 \\
\end{array}$ & & \\
\hline \multirow{2}{*}{2000} & 32 & 408 & 134 & 44 & 13 & 7 & 64 & 638 \\
\hline & $5,0 \%$ & $63,9 \%$ & $21,0 \%$ & $6,9 \%$ & $2,0 \%$ & $1,1 \%$ & $10,0 \%$ & $100,0 \%$ \\
\hline \multirow{2}{*}{2001} & 60 & 841 & 273 & 80 & 34 & 20 & 134 & 1308 \\
\hline & $4,6 \%$ & $64,3 \%$ & $20,9 \%$ & $6,1 \%$ & $2,6 \%$ & $1,5 \%$ & $10,2 \%$ & $100,0 \%$ \\
\hline \multirow{2}{*}{2002} & 65 & 893 & 214 & 90 & 27 & 17 & 134 & 1306 \\
\hline & $5,0 \%$ & $68,4 \%$ & $16,4 \%$ & $6,9 \%$ & $2,1 \%$ & $1,3 \%$ & $10,3 \%$ & $100,0 \%$ \\
\hline \multirow{2}{*}{2003} & 81 & 940 & 301 & 93 & 32 & 24 & 149 & 1471 \\
\hline & $5,5 \%$ & $63,9 \%$ & $20,5 \%$ & $6,3 \%$ & $2,2 \%$ & $1,6 \%$ & $10,1 \%$ & $100,0 \%$ \\
\hline \multirow{2}{*}{2004} & 101 & 1136 & 304 & 97 & 38 & 20 & 155 & 1696 \\
\hline & $6,0 \%$ & $67,0 \%$ & $17,9 \%$ & $5,7 \%$ & $2,2 \%$ & $1,2 \%$ & $9,1 \%$ & $100,0 \%$ \\
\hline \multirow{2}{*}{2005} & 95 & 1123 & 343 & 107 & 44 & 24 & 175 & 1736 \\
\hline & $5,5 \%$ & $64,7 \%$ & $19,8 \%$ & $6,2 \%$ & $2,5 \%$ & $1,4 \%$ & $10,1 \%$ & $100,0 \%$ \\
\hline \multirow{2}{*}{2006} & 94 & 1162 & 323 & 129 & 55 & 18 & 202 & 1781 \\
\hline & $5,3 \%$ & $65,2 \%$ & $18,1 \%$ & $7,2 \%$ & $3,1 \%$ & $1,0 \%$ & $11,3 \%$ & $100,0 \%$ \\
\hline \multirow{2}{*}{2007} & 88 & 1193 & 339 & 126 & 35 & 22 & 183 & 1803 \\
\hline & $4,9 \%$ & $66,2 \%$ & $18,8 \%$ & $7,0 \%$ & $1,9 \%$ & $1,2 \%$ & $10,1 \%$ & $100,0 \%$ \\
\hline \multirow{2}{*}{2008} & 90 & 1240 & 381 & 116 & 43 & 21 & 180 & 1891 \\
\hline & $4,8 \%$ & $65,6 \%$ & $20,1 \%$ & $6,1 \%$ & $2,3 \%$ & $1,1 \%$ & $9,5 \%$ & $100,0 \%$ \\
\hline \multirow{2}{*}{2009} & 98 & 1232 & 353 & 109 & 48 & 24 & 181 & 1864 \\
\hline & $5,3 \%$ & $66,1 \%$ & $18,9 \%$ & $5,8 \%$ & $2,6 \%$ & $1,3 \%$ & $9,7 \%$ & $100,0 \%$ \\
\hline \multirow{2}{*}{2010} & 93 & 1236 & 373 & 143 & 62 & 19 & 224 & 1926 \\
\hline & $4,8 \%$ & $64,2 \%$ & $19,4 \%$ & $7,4 \%$ & $3,2 \%$ & $1,0 \%$ & $11,6 \%$ & $100,0 \%$ \\
\hline \multirow{2}{*}{2011} & 92 & 1232 & 362 & 144 & 63 & 39 & 246 & 1932 \\
\hline & $4,8 \%$ & $63,8 \%$ & $18,7 \%$ & $7,5 \%$ & $3,3 \%$ & $2,0 \%$ & $12,7 \%$ & $100,0 \%$ \\
\hline \multirow{2}{*}{2012} & 88 & 1490 & 491 & 178 & 50 & 36 & 264 & 2333 \\
\hline & $3,8 \%$ & $63,9 \%$ & $21,0 \%$ & $7,6 \%$ & $2,1 \%$ & $1,5 \%$ & $11,3 \%$ & $100,0 \%$ \\
\hline \multirow{2}{*}{2013} & 118 & 1473 & 471 & 168 & 85 & 36 & 289 & 2351 \\
\hline & $5,0 \%$ & $62,7 \%$ & $20,0 \%$ & $7,1 \%$ & $3,6 \%$ & $1,5 \%$ & $12,3 \%$ & $100,0 \%$ \\
\hline \multirow{2}{*}{2014} & 105 & 1579 & 508 & 177 & 57 & 40 & 274 & 2466 \\
\hline & $4,3 \%$ & $64,0 \%$ & $20,6 \%$ & $7,2 \%$ & $2,3 \%$ & $1,6 \%$ & $11,1 \%$ & $100,0 \%$ \\
\hline \multirow{2}{*}{2015} & 126 & 1631 & 571 & 208 & 88 & 54 & 350 & 2678 \\
\hline & $4,7 \%$ & $60,9 \%$ & $21,3 \%$ & $7,8 \%$ & $3,3 \%$ & $2,0 \%$ & $13,1 \%$ & $100,0 \%$ \\
\hline
\end{tabular}




\subsection{Maternale Charakteristika}

\subsubsection{Alter der Mutter}

Der Mittelwert des mütterlichen Alters bei der Entbindung lag in den anhand des BMI eingeteilten Subgruppen bei Werten zwischen 31,0 und 31,5 Jahren. Normalgewichtige Frauen waren am Entbindungstermin im Mittel 31,2 Jahre alt (SD 5,2), übergewichtigen Frauen 31,4 Jahre (SD 5,3) und Studienteilnehmerinnen mit einem BMI $\geq 30 \mathrm{~kg} / \mathrm{m}^{2} 31,2$ Jahre alt (SD 5,4). Innerhalb des untersuchten Studienkollektivs (N=27754) betrug das Alter der Gebärenden im Mittel 31,2 Jahre (SD 5,3).

Die einfaktorielle Varianzanalyse wies auf einen signifikanten Unterschied zwischen den BMIGruppen hin $(\mathrm{p}=0,017)$. Zwischen normalgewichtigen und adipösen Frauen in ihrer Gesamtheit zeigten die paarweisen Mehrfachvergleiche keinen statistisch signifikanten Unterschied der Mittelwerte ( $\mathrm{p}=0,917)$, ebenso bei der Gegenüberstellung der Subgruppen von Frauen mit Adipositas Grad I bis III mit der Referenzgruppe ( $\mathrm{p}=0,888 ; \mathrm{p}=0,301 ; \mathrm{p}=0,161)$. Beim Vergleich von normalgewichtigen mit übergewichtigen Frauen konnte jedoch ein statistisch signifikanter Unterschied ( $\mathrm{p}=0,003)$ zwischen den Kategorien festgestellt werden (Tab. 4).

Tab. 4 Mittelwert und Standardabweichung des mütterlichen Alters bei Entbindung in Bezug auf BMI-Kategorien BMI-Kategorien $\left[\mathrm{kg} / \mathrm{m}^{2}\right]$

\begin{tabular}{lcccccccc} 
Alter der & $\mathbf{1 8 , 5 - 2 4 , 9}$ & $\mathbf{2 5 , 0 - 2 9 , 9}$ & $\mathbf{3 0 , 0 - 3 4 , 9}$ & $\mathbf{3 5 , 0 - 3 9 , 9}$ & $\mathbf{2 4 0}$ & $\mathbf{2 3 0}$ & Gesamt \\
Mutter [Jahre] & $\mathrm{n}=18809$ & $\mathrm{n}=5741$ & $\mathrm{n}=2009$ & $\mathrm{n}=774$ & $\mathrm{n}=421$ & $\mathrm{n}=3204$ & $\mathrm{~N}=27754$ \\
\hline \hline Mittelwert & 31,2 & 31,4 & 31,2 & 31,0 & 31,5 & 31,2 & 31,2 \\
\hline SD & 5,2 & 5,3 & 5,4 & 5,4 & 5,3 & 5,4 & 5,3 \\
\hline
\end{tabular}

\subsubsection{Verteilung der Parität}

Insgesamt war der Anteil von Primiparae mit 50,1\% und Pluriparae mit 49,9\% im Untersuchungszeitraum in etwa gleich. Bei der Analyse in Abhängigkeit des mütterlichen BMI war unter Anwendung des Chi-Quadrat-Tests festzustellen, dass der Anteil von Primiparae sowohl unter übergewichtigen mit 44,7\% als auch unter adipösen Müttern mit 41,6\% signifikant niedriger war (jeweils $p<0,001$ ) im Vergleich zur Referenzgruppe mit 53,2\%. Umgekehrt befanden sich unter den übergewichtigen Frauen (55,3\%) und unter den Schwangeren der Kategorie Adipositas $(58,4 \%)$ signifikant häufiger Pluriparae (jeweils $\mathrm{p}<0,001)$ als unter den normalgewichtigen Frauen mit 46,8\% (Tab. 5; Abb. 6). 
Tab. 5 Absolute und relative Häufigkeiten von Primi- und Pluriparae in Bezug auf maternale BMI-Kategorien

\begin{tabular}{|c|c|c|c|c|c|c|c|}
\hline \multirow[b]{3}{*}{ Parität } & \multicolumn{6}{|c|}{ BMI-Kategorien $\left[\mathrm{kg} / \mathrm{m}^{2}\right]$} & \multirow{3}{*}{$\begin{array}{c}\text { Gesamt } \\
\mathrm{N}=27754\end{array}$} \\
\hline & $18,5-24,9$ & $25,0-29,9$ & $30,0-34,9$ & $35,0-39,9$ & $\geq 40$ & $\geq \mathbf{3 0}$ & \\
\hline & $\mathrm{n}=18809$ & $\mathrm{n}=5741$ & $n=2009$ & $\mathrm{n}=774$ & $\mathrm{n}=421$ & $n=3204$ & \\
\hline \multirow{2}{*}{ Primipara } & 10007 & 2567 & 864 & 311 & 175 & 1332 & 13906 \\
\hline & $53,2 \%$ & $44,7 \%$ & $42,1 \%$ & $40,2 \%$ & $41,6 \%$ & $41,6 \%$ & $50,1 \%$ \\
\hline \multirow{2}{*}{ Pluripara } & 8802 & 3174 & 1163 & 463 & 246 & 1872 & 13848 \\
\hline & $46,8 \%$ & $55,3 \%$ & $57,9 \%$ & $59,8 \%$ & $58,4 \%$ & $58,4 \%$ & $49,9 \%$ \\
\hline
\end{tabular}

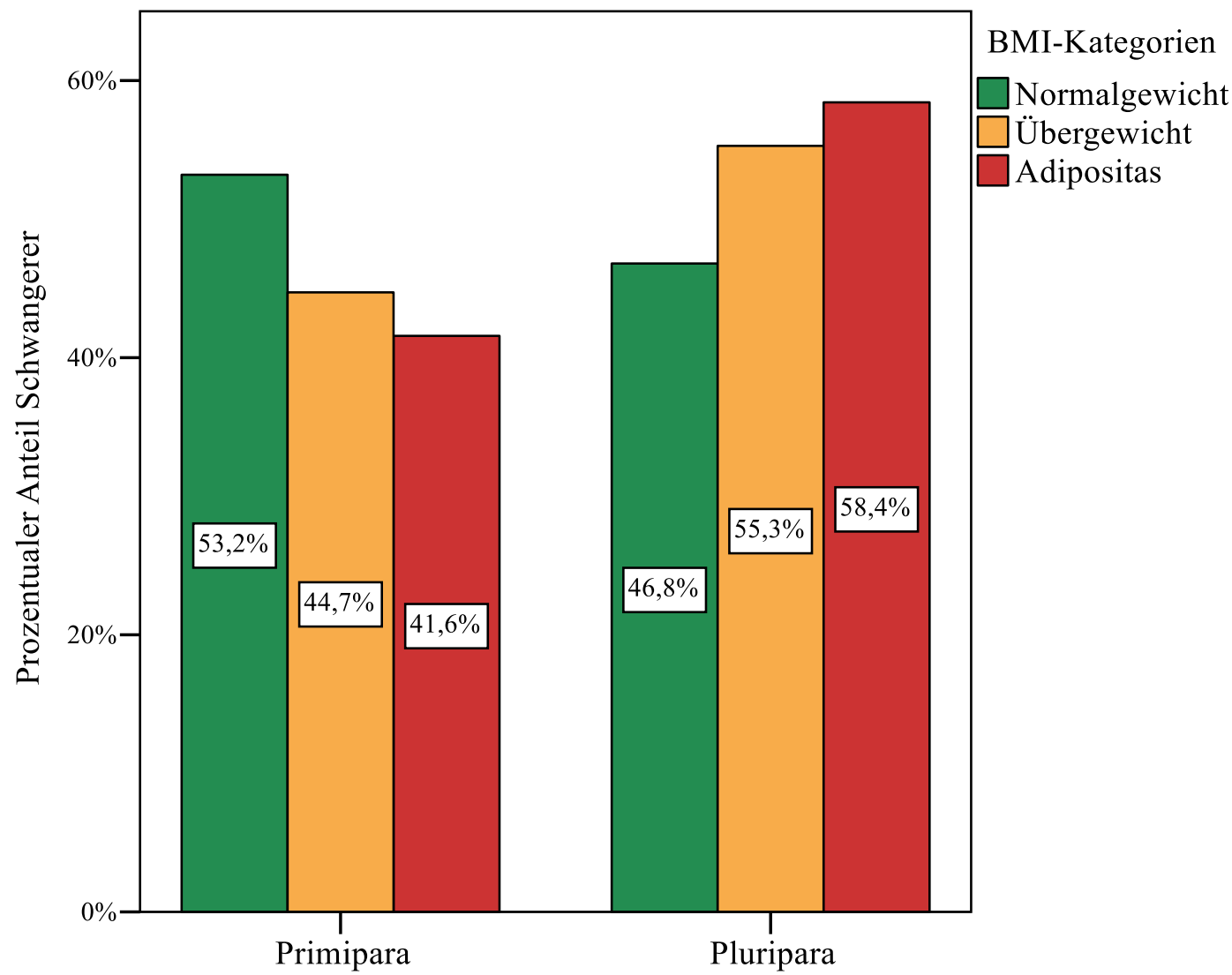

Parität

Abb. 6 Relative Häufigkeitsverteilung von Primi- und Pluriparae in Bezug auf maternale BMI-Kategorien $(N=27754)$

\subsubsection{Aborte in früheren Schwangerschaften}

Insgesamt waren von den Studienteilnehmerinnen, die im Untersuchungszeitraum in der Klinik St. Hedwig entbanden, 4163 Frauen (15,0\%) von einem Abort und 1418 Frauen (5,1\%) von zwei oder mehr Aborten in früheren Schwangerschaften betroffen gewesen. In beiden Punkten war eine signifikante Assoziation mit dem mütterlichen BMI zu beobachten. 
Innerhalb der BMI-Kategorie Normalgewicht betrug die Rate von Frauen mit einem stattgehabten Abort 14,5\%. Im Vergleich dazu war der Anteil unter den übergewichtigen mit 15,7\% und unter den adipösen Patientinnen mit 16,8\% höher, was der Chi-Quadrat-Test als statistisch signifikant bestätigte ( $\mathrm{p}=0,005$ bzw. $\mathrm{p}<0,001)$. Zudem kam hervor, dass die relative Häufigkeit von Frauen mit einer erlittenen Fehlgeburt mit steigender BMI-Kategorie zunahm. Der Prozentsatz betrug unter Frauen mit Adipositas Grad I und II 15,8\% bzw. 15,9\% und erreichte unter Frauen mit einem BMI $\geq 40$ kg/m² ein Maximum von 23,3\% (Abb. 7; Tab. 6).

Der prozentuale Anteil der Patientinnen mit bereits zwei oder mehr dokumentierten Aborten in vergangenen Schwangerschaften war ebenfalls unter übergewichtigen Frauen mit 6,0\% und unter adipösen Studienteilnehmerinnen mit 6,4\% signifikant höher (jeweils $p<0,001$, ChiQuadrat-Test) als unter den Frauen des Referenzkollektivs mit 4,6\% (Abb. 7; Tab. 6).

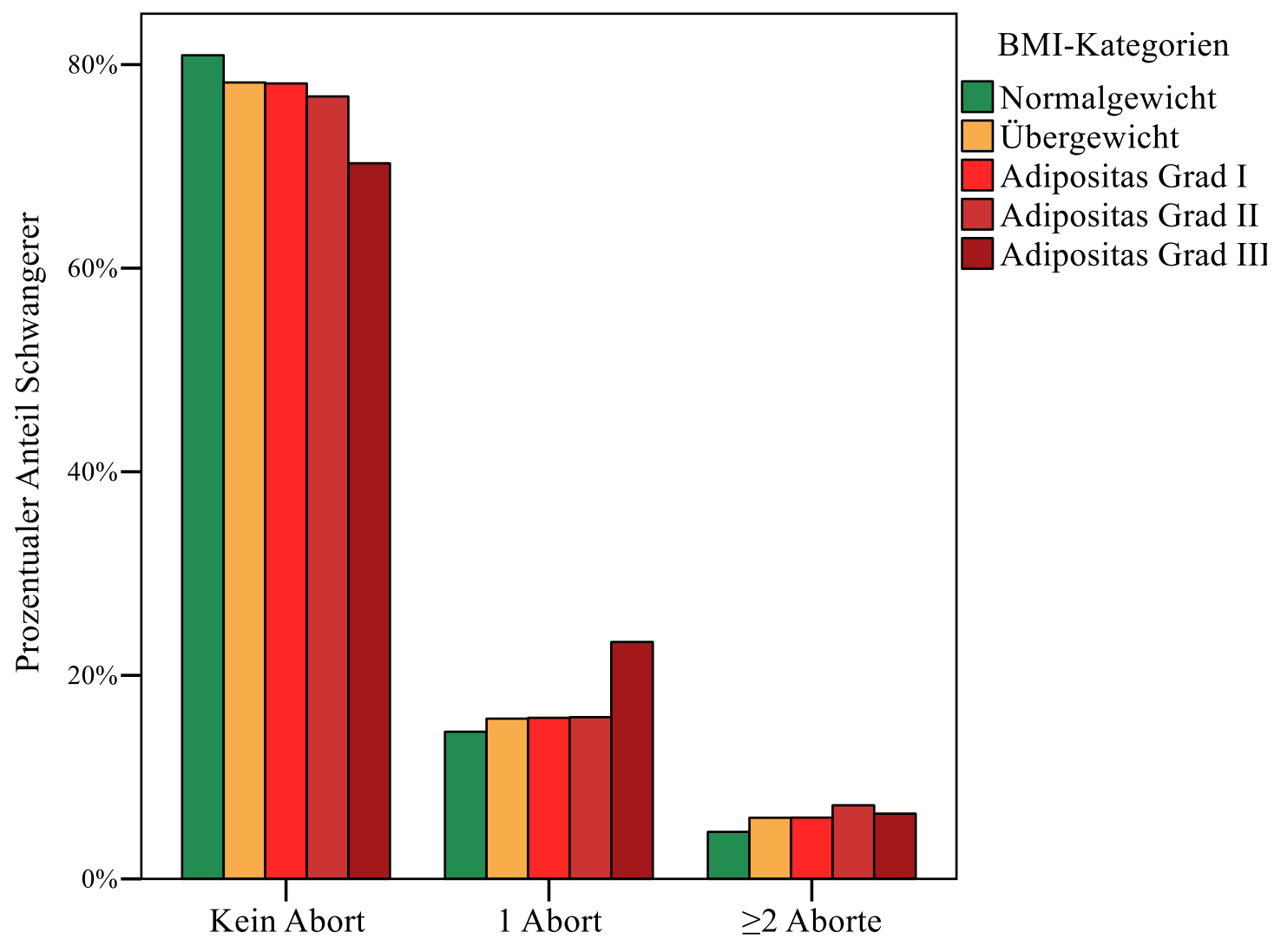

Aborte in früheren Schwangerschaften

Abb. 7 Relative Häufigkeitsverteilung der klassifizierten Anzahl stattgehabter Aborte in früheren Schwangerschaften in Bezug auf maternale BMI-Kategorien $(N=27754)$ 
Tab. 6 Absolute und relative Häufigkeit der klassifizierten Anzahl stattgehabter Aborte in früheren Schwangerschaften in Bezug auf maternale BMI-Kategorien

BMI-Kategorien $\left[\mathrm{kg} / \mathrm{m}^{2}\right]$

\begin{tabular}{|c|c|c|c|c|c|c|c|}
\hline Aborte & $\begin{array}{c}\mathbf{1 8 , 5 - 2 4 , 9} \\
\mathrm{n}=18809\end{array}$ & $\begin{array}{c}\mathbf{2 5 , 0 - 2 9 , 9} \\
n=5741\end{array}$ & $\begin{array}{c}\mathbf{3 0 , 0 - 3 4 , 9} \\
n=2009\end{array}$ & $\begin{array}{c}\mathbf{3 5 , 0 - 3 9 , 9} \\
\mathrm{n}=774\end{array}$ & $\begin{array}{c}\geq \mathbf{4 0} \\
\mathrm{n}=421\end{array}$ & $\begin{array}{c}\geq \mathbf{3 0} \\
n=3204\end{array}$ & $\begin{array}{c}\text { Gesamt } \\
\mathrm{N}=27754\end{array}$ \\
\hline \multirow{2}{*}{ Keiner } & (15220 & $\overline{4492}$ & 1570 & 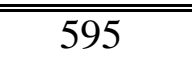 & 206 & 2461 & 22173 \\
\hline & $80,9 \%$ & $78,2 \%$ & $78,1 \%$ & $76,9 \%$ & $70,3 \%$ & $76,8 \%$ & $79,9 \%$ \\
\hline \multirow{2}{*}{1 Abort } & 2720 & 904 & 318 & 123 & 98 & 539 & 4163 \\
\hline & $14,5 \%$ & $15,7 \%$ & $15,8 \%$ & $15,9 \%$ & $23,3 \%$ & $16,8 \%$ & $15,0 \%$ \\
\hline \multirow{2}{*}{$\geq 2$ Aborte } & 869 & 345 & 121 & 56 & 27 & 204 & 1418 \\
\hline & $4,6 \%$ & $6,0 \%$ & $6,0 \%$ & $7,2 \%$ & $6,4 \%$ & $6,4 \%$ & $5,1 \%$ \\
\hline
\end{tabular}

Bei der in Tabelle 7 dargestellten Analyse der Subgruppe von Frauen nach Ausschluss der Fälle eines Diabetes mellitus in der Schwangerschaft $(\mathrm{N}=25335)$ konnten die bereits festgestellten Zusammenhänge zwischen dem mütterlichen BMI und der erhöhten Abortrate bestätigt werden, was für einen unabhängigen Einfluss der Adipositas von einer diabetischen Stoffwechsellage der Frau auf das Abortrisiko sprach.

Insgesamt war der Anteil der Frauen mit einer Fehlgeburt (14,8\%) sowie mit zwei oder mehr Fehlgeburten (4,9\%) in vergangenen Schwangerschaften in dieser Subgruppenanalyse etwas niedriger als bei der Auswertung im Gesamtkollektiv. Übergewichtige Frauen mit 15,5\% bzw. 5,9\% und adipöse Frauen mit 16,5\% bzw. 5,7\% hatten signifikant häufiger bereits einen ( $p=0,009$ bzw. $p=0,001)$ oder mehrere Aborte ( $p<0,001$ bzw. $p=0,002)$ in früheren Schwangerschaften erlitten als normalgewichtige Frauen mit 14,3\% bzw. 4,4\% (Tab. 7).

Tab. 7 Absolute und relative Häufigkeiten der klassifizierten Anzahl stattgehabter Aborte in früheren Schwangerschaften in Bezug auf maternale BMI-Kategorien in der Subgruppe stoffwechselgesunder Frauen

BMI-Kategorien $\left[\mathrm{kg} / \mathrm{m}^{2}\right]$

\begin{tabular}{lccccccc} 
& $\mathbf{1 8 , 5 - 2 4 , 9}$ & $\mathbf{2 5 , 0 - 2 9 , 9}$ & $\mathbf{3 0 , 0 - 3 4 , 9}$ & $\mathbf{3 5 , 0 - 3 9 , 9}$ & $\mathbf{2 4 0}$ & $\mathbf{2 3 0}$ & \multirow{2}{*}{ Gesamt } \\
\cline { 2 - 6 } Aborte & $\mathrm{n}=17689$ & $\mathrm{n}=5124$ & $\mathrm{n}=1668$ & $\mathrm{n}=572$ & $\mathrm{n}=282$ & $\mathrm{n}=2522$ & $\mathrm{~N}=25335$ \\
\hline \hline \multirow{2}{*}{ Keiner } & 14377 & 4025 & 1320 & 442 & 200 & 1962 & 20364 \\
& $81,3 \%$ & $78,6 \%$ & $79,1 \%$ & $77,3 \%$ & $70,9 \%$ & $77,8 \%$ & $80,4 \%$ \\
\hline \multirow{2}{*}{ Abort } & 2529 & 795 & 253 & 94 & 70 & 417 & 3741 \\
& $14,3 \%$ & $15,5 \%$ & $15,2 \%$ & $16,4 \%$ & $24,8 \%$ & $16,5 \%$ & $14,8 \%$ \\
\hline \multirow{2}{*}{ 22 Aborte } & 783 & 304 & 95 & 36 & 12 & 143 & 1230 \\
& $4,4 \%$ & $5,9 \%$ & $5,7 \%$ & $6,3 \%$ & $4,3 \%$ & $5,7 \%$ & $4,9 \%$ \\
\hline
\end{tabular}




\subsubsection{Chronische Erkrankungen}

In Bezug auf das gesamte Studienkollektiv waren 1,2\% der Frauen (342 Fälle) bereits vor der Schwangerschaft an Diabetes mellitus erkrankt. Hinsichtlich der maternalen Gewichtsklasse war der Anteil der Mütter mit präexistentem Diabetes mellitus unter übergewichtigen und adipösen Patientinnen mit 1,9\% bzw. 3,4\% signifikant höher als unter denen des Referenzkollektivs mit 0,6\% (jeweils p<0,001, Chi-Quadrat-Test). Zudem konnte eine Zunahme der DiabetesFälle mit steigendem BMI der Schwangeren beobachtet werden. Der prozentuale Anteil stieg ausgehend von $0,6 \%$ unter normalgewichtigen Frauen auf 1,9\% unter übergewichtigen Patientinnen an, unter Frauen mit Adipositas Grad I und II auf 2,4\% bzw. 4,3\% und erreichte unter Müttern mit Adipositas Grad III einen Höchstwert von 6,7\% (Tab. 8; Abb. 8).

Tab. 8 Absolute und relative Häufigkeiten eines präexistenten Diabetes mellitus in Bezug auf maternale BMIKategorien

BMI-Kategorien $\left[\mathrm{kg} / \mathrm{m}^{2}\right]$

\begin{tabular}{lccccccc} 
Diabetes & $\mathbf{1 8 , 5 - 2 4 , 9}$ & $\mathbf{2 5 , 0 - 2 9 , 9}$ & $\mathbf{3 0 , 0 - 3 4 , 9}$ & $\mathbf{3 5 , 0 - 3 9 , 9}$ & $\mathbf{2 4 0}$ & $\mathbf{2 3 0}$ & Gesamt \\
mellitus & $\mathrm{n}=18809$ & $\mathrm{n}=5741$ & $\mathrm{n}=2009$ & $\mathrm{n}=774$ & $\mathrm{n}=421$ & $\mathrm{n}=3204$ & $\mathrm{~N}=27754$ \\
\hline \hline \multirow{2}{*}{ Ja } & 122 & 111 & 48 & 33 & 28 & 109 & 342 \\
& $0,6 \%$ & $1,9 \%$ & $2,4 \%$ & $4,3 \%$ & $6,7 \%$ & $3,4 \%$ & $1,2 \%$ \\
\hline \multirow{2}{*}{ Nein } & 18687 & 5630 & 1961 & 741 & 393 & 3095 & 27412 \\
& $99,4 \%$ & $98,1 \%$ & $97,6 \%$ & $95,7 \%$ & $93,3 \%$ & $96,6 \%$ & $98,8 \%$ \\
\hline
\end{tabular}




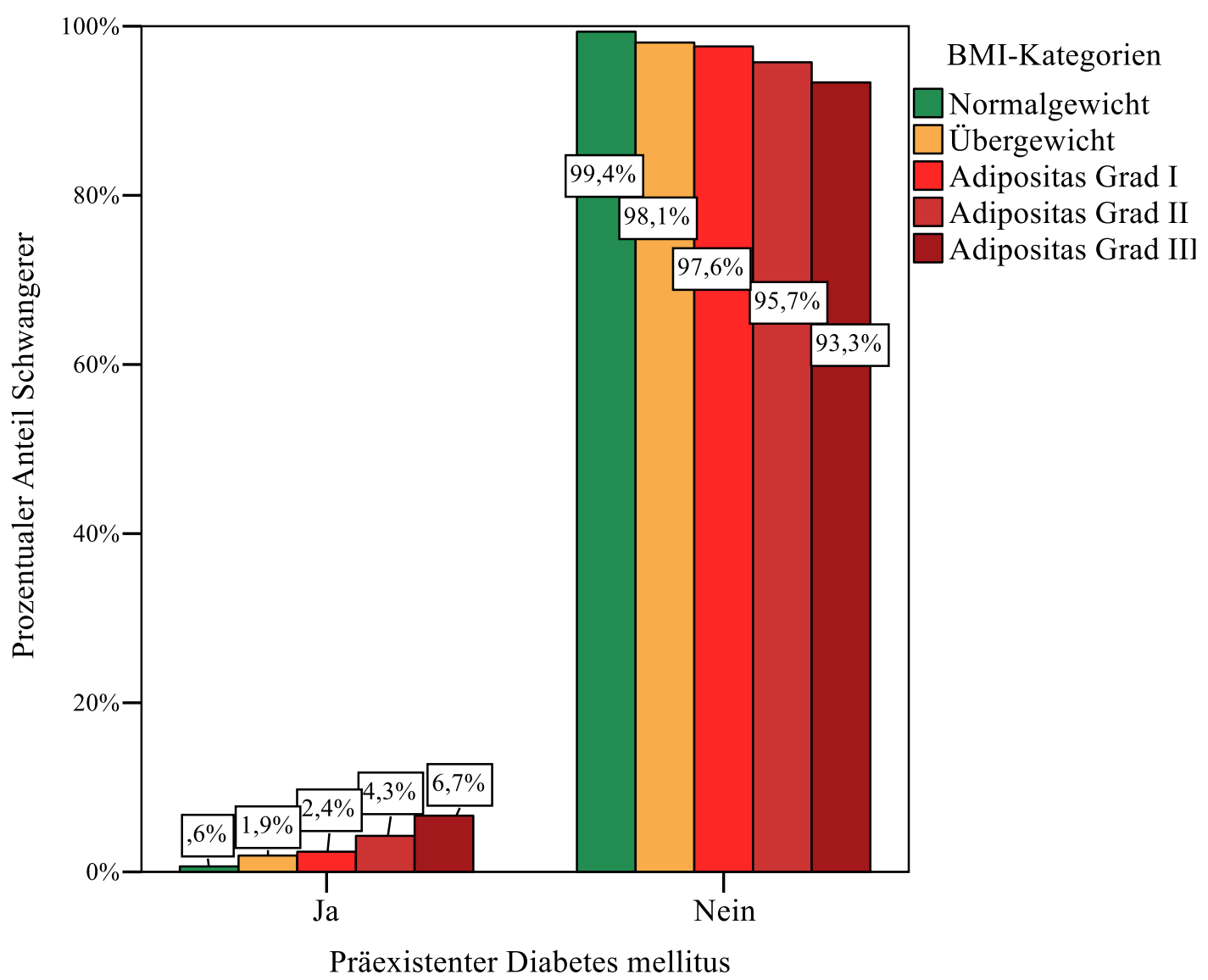

Abb. 8 Relative Häufigkeitsverteilung des präexistenten Diabetes mellitus in Bezug auf maternale BMI-Kategorien $(N=27754)$

Zudem waren übergewichtige und adipöse Patientinnen mit 0,2\% (14 Fälle) bzw. 1,1\% (35 Fälle) signifikant häufiger (jeweils $\mathrm{p}<0,001$ ) schon vor der Schwangerschaft an Hypertonie erkrankt als die Frauen mit Normalgewicht mit 0,1\% (11 Fälle). Insgesamt traten im Zeitraum der Analyse 60 Fälle (0,2\%) einer bereits vor der Schwangerschaft bestehenden Hypertonie auf. Auch hier konnte eine Tendenz steigender Werte mit Zunahme der mütterlichen BMI-Kategorie beobachtet werden. Der Anteil stieg ausgehend von 0,1\% unter normalgewichtigen Frauen auf 0,2\% unter übergewichtigen Frauen an sowie innerhalb der Gruppe der adipösen Patientinnen der Schweregrade I bis III weiter auf $0,8 \%, 1,2 \%$ und 2,4\% (Tab. 9).

Tab. 9 Absolute und relative Häufigkeiten einer chronischen Hypertonie in Bezug auf maternale BMI-Kategorien

BMI-Kategorien $\left[\mathrm{kg} / \mathrm{m}^{2}\right]$

\begin{tabular}{|c|c|c|c|c|c|c|c|}
\hline $\begin{array}{l}\text { Chronische } \\
\text { Hypertonie }\end{array}$ & $\begin{array}{c}\mathbf{1 8 , 5}-24,9 \\
n=18809\end{array}$ & $\begin{array}{c}\mathbf{2 5 , 0 - 2 9 , 9} \\
n=5741\end{array}$ & $\begin{array}{c}\mathbf{3 0 , 0 - 3 4 , 9} \\
n=2009\end{array}$ & $\begin{array}{c}\mathbf{3 5 , 0 - 3 9 , 9} \\
n=774\end{array}$ & $\begin{array}{c}\geq \mathbf{4 0} \\
\mathrm{n}=421\end{array}$ & $\begin{array}{c}\geq \mathbf{3 0} \\
n=3204\end{array}$ & $\begin{array}{c}\text { Gesamt } \\
\mathrm{N}=27754\end{array}$ \\
\hline \multirow{2}{*}{$\mathrm{Ja}$} & 11 & 14 & 16 & 9 & 10 & 35 & 60 \\
\hline & $0,1 \%$ & $0,2 \%$ & $0,8 \%$ & $1,2 \%$ & $2,4 \%$ & $1,1 \%$ & $0,2 \%$ \\
\hline \multirow{2}{*}{ Nein } & 18798 & 5727 & 1993 & 765 & 411 & 3169 & 27694 \\
\hline & $99,9 \%$ & $99,8 \%$ & $99,2 \%$ & $98,8 \%$ & $97,6 \%$ & $98,9 \%$ & $99,8 \%$ \\
\hline
\end{tabular}




\subsubsection{Gewichtszunahme während der Schwangerschaft}

Die mittlere Gewichtszunahme der Patientinnen während der Schwangerschaft betrug im Gesamtkollektiv 13,7 kg (SD 5,8). Unter Einbeziehung des mütterlichen BMI zeigte sich, dass übergewichtige und adipöse Frauen im Mittel mit 13,6 kg (SD 6,1) bzw. 10,7 kg (SD 7,2) weniger an Gewicht während der Schwangerschaft zunahmen als normalgewichtige Frauen mit 14,3 kg (SD 5,2). Die Unterschiede zwischen den BMI-Gruppen bestätigte die einfaktorielle Varianzanalyse bzw. die paarweisen Mehrfachvergleiche jeweils mit $p<0,001$ als statistisch signifikant (Tab. 10).

Tab. 10 Mittelwert und Standardabweichung der maternalen Gewichtszunahme während der Schwangerschaft in Bezug auf BMI-Kategorien

BMI-Kategorien $\left[\mathrm{kg} / \mathrm{m}^{2}\right]$

\begin{tabular}{|c|c|c|c|c|c|c|c|}
\hline $\begin{array}{l}\text { Gewichts- } \\
\text { zunahme [kg] }\end{array}$ & $\begin{array}{c}\mathbf{1 8 , 5 - 2 4 , 9} \\
\mathrm{n}=17844\end{array}$ & $\begin{array}{c}\mathbf{2 5 , 0 - 2 9 , 9} \\
n=5400\end{array}$ & $\begin{array}{c}\mathbf{3 0 , 0 - 3 4 , 9} \\
n=1879\end{array}$ & $\begin{array}{c}35,0-39,9 \\
n=728\end{array}$ & $\begin{array}{c}\geq \mathbf{4 0} \\
n=381\end{array}$ & $\begin{array}{c}\geq \mathbf{3 0} \\
\mathrm{n}=2988\end{array}$ & $\begin{array}{c}\text { Gesamt } \\
\mathrm{N}=26232\end{array}$ \\
\hline Mittelwert & 14,3 & 13,6 & 11,5 & 9,9 & 8,4 & 10,7 & 13,7 \\
\hline SD & 5,2 & 6,1 & 6,8 & 7,2 & 8,0 & 7,2 & 5,8 \\
\hline
\end{tabular}

Die vom IOM empfohlene Gewichtszunahme in der Schwangerschaft, die sich am mütterlichen BMI orientiert, wurde im Untersuchungszeitraum insgesamt in 44,1\% der Fälle überschritten, entsprach in 30,7\% der Empfehlung und wurde in 25,2\% der Fälle unterschritten (Tab. 11).

Eine höhere Gewichtszunahme als vom IOM empfohlen wurde unter 62,4\% der übergewichtigen und 59,1\% der adipösen Schwangeren beobachtet, was als signifikant häufiger im Vergleich zu normalgewichtigen Frauen mit einem Anteil von 36,1\% bestätigt wurde (jeweils $\mathrm{p}<0,001)$. Des Weiteren entsprach die Gewichtszunahme der übergewichtigen und adipösen Frauen mit 24,1\% bzw. 20,5\% signifikant seltener dem empfohlenen Bereich des IOM als bei den Frauen der Referenzgruppe mit 34,4\% (jeweils p<0,001). Eine Unterschreitung der angeratenen Gewichtszunahme konnte unter normalgewichtigen Frauen mit 29,5\% signifikant häufiger festgestellt werden als unter übergewichtigen und adipösen Studienteilnehmerinnen mit 13,5\% bzw. 20,4\% (jeweils p<0,001).

Die bivariate Analyse erfolgte in allen Punkten über den Chi-Quadrat-Test.

Die absoluten und relativen Häufigkeiten einer Einhaltung sowie Über- und Unterschreitung der empfohlenen Gewichtszunahme des IOM verdeutlichen Tabelle 11 und Abbildung 9. 
Tab. 11 Absolute und relative Häufigkeiten einer Einhaltung sowie Unter- und Überschreitung der empfohlenen Gewichtszunahme des IOM in Bezug auf maternale BMI-Kategorien

BMI-Kategorien $\left[\mathrm{kg} / \mathrm{m}^{2}\right]$

\begin{tabular}{|c|c|c|c|c|c|c|c|}
\hline $\begin{array}{l}\text { Empfehlung } \\
\text { IOM }\end{array}$ & $\begin{array}{c}\mathbf{1 8 , 5 - 2 4 , 9} \\
n=17844\end{array}$ & $\begin{array}{c}\mathbf{2 5 , 0 - 2 9 , 9} \\
n=5400\end{array}$ & $\begin{array}{c}\mathbf{3 0 , 0 - 3 4 , 9} \\
\mathrm{n}=1879\end{array}$ & $\begin{array}{c}35,0-39,9 \\
n=728\end{array}$ & $\begin{array}{c}\geq \mathbf{4 0} \\
\mathrm{n}=381\end{array}$ & $\begin{array}{c}\geq \mathbf{3 0} \\
n=2988\end{array}$ & $\begin{array}{c}\text { Gesamt } \\
\mathrm{N}=26232\end{array}$ \\
\hline \multirow{2}{*}{ Unterschritten } & 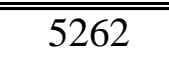 & 729 & 284 & 185 & $14 \overline{140}$ & (609 & 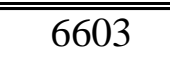 \\
\hline & $29,5 \%$ & $13,5 \%$ & $15,1 \%$ & $25,4 \%$ & $36,7 \%$ & $20,4 \%$ & $25,2 \%$ \\
\hline \multirow{2}{*}{ Eingehalten } & 6145 & 1304 & 380 & 162 & 71 & 613 & 8062 \\
\hline & $34,4 \%$ & $24,1 \%$ & $20,2 \%$ & $22,3 \%$ & $18,6 \%$ & $20,5 \%$ & $30,7 \%$ \\
\hline \multirow{2}{*}{ Überschritten } & 6434 & 3367 & 1215 & 381 & 170 & 1766 & 11567 \\
\hline & $36,1 \%$ & $62,4 \%$ & $64,7 \%$ & $52,3 \%$ & $44,6 \%$ & $59,1 \%$ & $44,1 \%$ \\
\hline
\end{tabular}

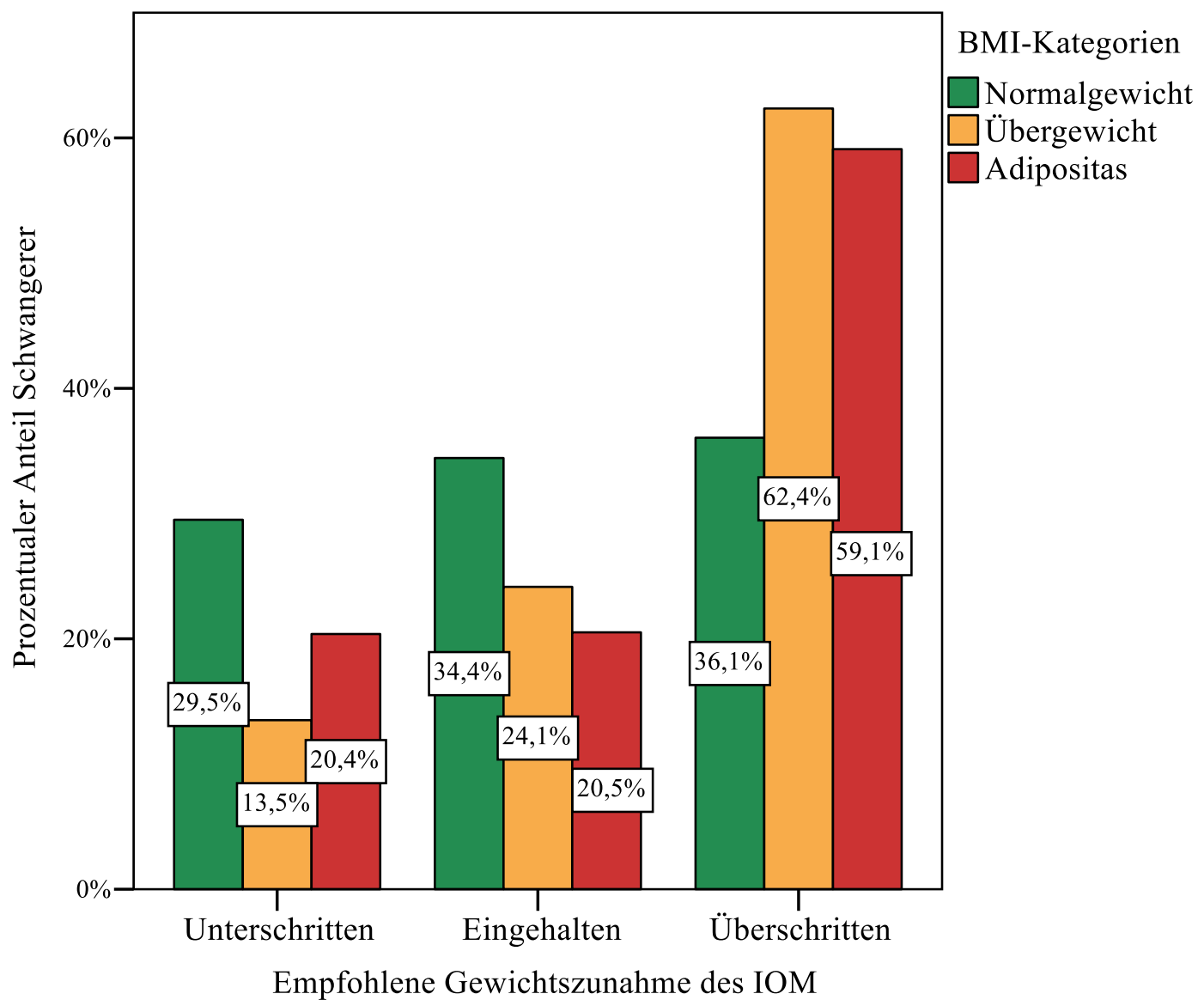

Abb. 9 Relative Häufigkeitsverteilung der Einhaltung sowie Unter- und Überschreitung der empfohlenen Gewichtszunahme des IOM in Bezug auf maternale BMI-Kategorien (N=26232) 


\subsubsection{Aufenthaltsdauer in der Klinik}

Auch hinsichtlich der mittleren Aufenthaltsdauer der Patientinnen im Krankenhaus konnten signifikante Unterschiede zwischen den verschiedenen BMI-Gruppen beobachtet werden, wobei diese im Gesamtkollektiv bei 4,5 Tagen (SD 4,3) lag.

Die mittlere Anzahl der verbrachten Tage in der Klinik war mit insgesamt 4,7 Tagen (SD 4,4) in der BMI-Kategorie Übergewicht bzw. 5,1 Tagen (SD 5,3) in der BMI-Kategorie Adipositas signifikant länger im Vergleich zu normalgewichtigen Frauen, deren mittlere Aufenthaltsdauer 4,4 Tage (SD 4,1) betrug (jeweils p<0,001; ANOVA bzw. Post-hoc Tests). Die längste Dauer des Aufenthalts wurde in der BMI-Gruppe Adipositas Grad III beobachtet, deren zugehörige Frauen im Mittel 6,1 Tage (SD 7,2) in der Klinik blieben (Tab. 12).

Tab. 12 Mittelwert und Standardabweichung der mütterlichen Aufenthaltsdauer in der Klinik insgesamt in Bezug auf BMI-Kategorien

BMI-Kategorien $\left[\mathrm{kg} / \mathrm{m}^{2}\right]$

\begin{tabular}{lccccccc}
\cline { 2 - 6 } $\begin{array}{l}\text { Aufenthalts- } \\
\text { dauer [Tage] }\end{array}$ & $\begin{array}{c}\mathbf{1 8 , 5 - 2 4 , 9} \\
\mathrm{n}=18615\end{array}$ & $\begin{array}{c}\mathbf{2 5 , 0 - 2 9 , 9} \\
\mathrm{n}=5686\end{array}$ & $\begin{array}{c}\mathbf{3 0 , 0 - 3 4 , 9} \\
\mathrm{n}=1990\end{array}$ & $\begin{array}{c}\mathbf{3 5 , 0 - 3 9 , 9} \\
\mathrm{n}=765\end{array}$ & $\begin{array}{c}\mathbf{2 4 0} \\
\mathrm{n}=418\end{array}$ & $\begin{array}{c}\mathbf{2 3 0} \\
\mathrm{n}=3173\end{array}$ & $\begin{array}{c}\text { Gesamt } \\
\mathrm{N}=27475\end{array}$ \\
\hline \hline Mittelwert & 4,4 & 4,7 & 5,0 & 4,9 & 6,1 & 5,1 & 4,5 \\
\hline $\mathrm{SD}$ & 4,1 & 4,4 & 5,2 & 3,7 & 7,2 & 5,3 & 4,3 \\
\hline
\end{tabular}




\subsection{Schwangerschaftsverlauf}

\subsubsection{Gestationsdiabetes mellitus}

Im Untersuchungszeitraum erkrankten in der Klinik St. Hedwig insgesamt 2077 Schwangere an Gestationsdiabetes mellitus, was einem Anteil von 7,6\% entsprach. Von diesen 2077 Gestationsdiabetikerinnen gehörten 998 Frauen der Kategorie Normalgewicht an (5,3\%), 506 Frauen der Kategorie Übergewicht (9,0\%) und 573 Frauen der Gruppe Adipositas (18,5\%).

Dabei zeichnete sich unter Anwendung des Chi-Quadrat-Tests ab, dass mit jeder nächsthöheren BMI-Kategorie von Normalgewicht bis Adipositas Grad III der Anteil von Gestationsdiabetikerinnen signifikant zunahm. Während er unter normalgewichtigen Frauen 5,3\% betrug, stieg er bei übergewichtigen Patientinnen auf 9,0\% an, bei Schwangeren mit Adipositas Grad I und II auf $14,9 \%$ bzw. 22,8\% (jeweils p<0,001) und erreichte bei Frauen mit Adipositas Grad III einen Wert von 28,2\% ( $p=0,043)$. Patientinnen mit präexistentem Diabetes mellitus $(n=342)$ waren hierbei von der Analyse ausgeschlossen.

Die absoluten und relativen Häufigkeiten eines GDM sind in Tabelle 13 und Abbildung 10 in Bezug auf die mütterlichen BMI-Kategorien dargestellt.

Tab. 13 Absolute und relative Häufigkeiten eines Gestationsdiabetes mellitus in Bezug auf maternale BMI-Kategorien

\begin{tabular}{|c|c|c|c|c|c|c|c|}
\hline \multirow{3}{*}{$\begin{array}{l}\text { Gestations- } \\
\text { diabetes }\end{array}$} & \multicolumn{6}{|c|}{ BMI-Kategorien $\left[\mathrm{kg} / \mathrm{m}^{2}\right]$} & \multirow{3}{*}{$\begin{array}{c}\text { Gesamt } \\
\mathrm{N}=27412\end{array}$} \\
\hline & $18,5-24,9$ & $25,0-29,9$ & $30,0-34,9$ & $35,0-39,9$ & $\geq 40$ & $\geq \mathbf{3 0}$ & \\
\hline & $\mathrm{n}=18687$ & $\mathrm{n}=5630$ & $\mathrm{n}=1961$ & $\mathrm{n}=741$ & $n=393$ & $n=3095$ & \\
\hline \multirow{2}{*}{$\mathrm{Ja}$} & 998 & 506 & 293 & 169 & 111 & $\overline{573}$ & 2077 \\
\hline & $5,3 \%$ & $9,0 \%$ & $14,9 \%$ & $22,8 \%$ & $28,2 \%$ & $18,5 \%$ & $7,6 \%$ \\
\hline \multirow{2}{*}{ Nein } & 17689 & 5124 & 1668 & 572 & 282 & 2522 & 25335 \\
\hline & $94,7 \%$ & $91,0 \%$ & $85,1 \%$ & $77,2 \%$ & $71,8 \%$ & $81,5 \%$ & $92,4 \%$ \\
\hline
\end{tabular}




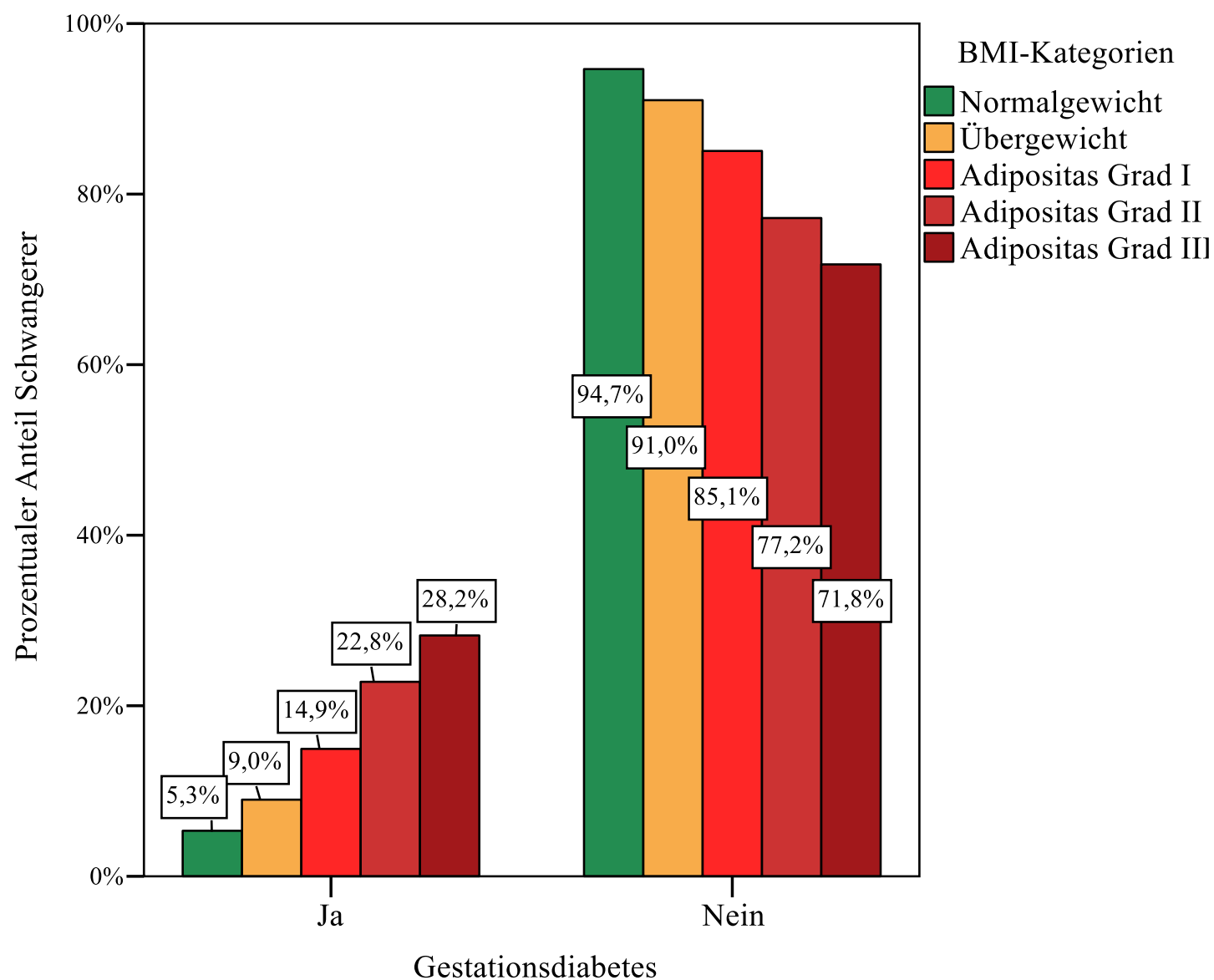

Abb. 10 Relative Häufigkeitsverteilung des Gestationsdiabetes mellitus in Bezug auf maternale BMI-Kategorien $(N=27412)$

Von den insgesamt 2077 Fällen eines Gestationsdiabetes mellitus traten zwei Fälle (1,5\%) in der Alterskategorie kleiner 18 Jahre auf, 1381 Fälle (6,7\%) innerhalb der 18 bis 35-jährigen Patientinnen und 694 Fälle (10,5\%) unter den Schwangeren, die bei der Entbindung älter als 35 Jahre waren (Tab. 14). Demnach stieg die Prävalenz des GDM mit der mütterlichen Altersgruppe an, was sich im Chi-Quadrat-Test als statistisch signifikant bestätigte $(p<0,001)$.

Bei der Betrachtung der GDM-Frequenz in Bezug auf die mütterliche BMI-Kategorie stratifiziert nach deren Altersgruppe zeigte sich, dass die Erkrankungshäufigkeit mit zunehmendem BMI in allen genannten Alterskategorien anstieg. Sowohl innerhalb der Subgruppe der unter 18-jährigen, der 18 bis 35-jährigen als auch der über 35-jährigen Frauen erkrankten adipöse Schwangere mit 20,0\%, 16,3\% und 25,1\% signifikant häufiger an Gestationsdiabetes mellitus als normalgewichtige Frauen mit $0,0 \%, 4,8 \%$ und 7,1\% (jeweils $\mathrm{p}<0,001$, Chi-Quadrat-Test). Die Prävalenz des GDM erreichte den höchsten Wert unter den Patientinnen der BMI-Kategorie Adipositas Grad III in der Altersgruppe über 35 Jahre mit 35,7\%. 
Somit war Adipositas in der Schwangerschaft, unabhängig von der maternalen Altersgruppe, signifikant mit einer Erhöhung der GDM-Rate assoziiert, wobei die Erkrankungshäufigkeit mit zunehmendem Alter der Mutter anstieg.

Tab. 14 Absolute und relative Häufigkeiten eines Gestationsdiabetes mellitus in Bezug auf maternale BMI-Kategorien, stratifiziert nach mütterlichen Altersgruppen

BMI-Kategorien $\left[\mathrm{kg} / \mathrm{m}^{2}\right]$

\begin{tabular}{|c|c|c|c|c|c|c|c|}
\hline $\begin{array}{l}\text { Gestations- } \\
\text { diabetes }\end{array}$ & $\begin{array}{c}\mathbf{1 8 , 5}-24,9 \\
\mathrm{n}=18687\end{array}$ & $\begin{array}{c}\mathbf{2 5 , 0 - 2 9 , 9} \\
\mathrm{n}=5630\end{array}$ & $\begin{array}{c}\mathbf{3 0 , 0 - 3 4 , 9} \\
\mathrm{n}=1961 \\
\end{array}$ & $\begin{array}{c}\mathbf{3 5 , 0 - 3 9 , 9} \\
n=741\end{array}$ & $\begin{array}{c}\geq 40 \\
n=393\end{array}$ & $\begin{array}{c}\geq \mathbf{3 0} \\
n=3095\end{array}$ & $\begin{array}{c}\text { Gesamt } \\
\mathrm{N}=27412\end{array}$ \\
\hline$<18$ Jahre & $\mathrm{n}=95$ & $n=28$ & $\mathrm{n}=8$ & $\mathrm{n}=2$ & $\mathrm{n}=0$ & $\mathrm{n}=10$ & $n=133$ \\
\hline \multirow{2}{*}{$\mathrm{Ja}$} & $\overline{00}$ & $\overline{00}$ & 2 & 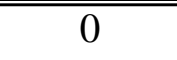 & $\overline{0}$ & 2 & 2 \\
\hline & $0,0 \%$ & $0,0 \%$ & $25,0 \%$ & $0,0 \%$ & $0,0 \%$ & $20,0 \%$ & $1,5 \%$ \\
\hline \multirow{2}{*}{ Nein } & 95 & 28 & 6 & 2 & 0 & 8 & 131 \\
\hline & $100,0 \%$ & $100,0 \%$ & $75,0 \%$ & $100,0 \%$ & $0,0 \%$ & $80,0 \%$ & $98,5 \%$ \\
\hline 18-35 Jahre & $\mathrm{n}=14241$ & $n=4130$ & $\mathrm{n}=1459$ & $\mathrm{n}=561$ & $\mathrm{n}=281$ & $\mathrm{n}=2301$ & $\mathrm{n}=20672$ \\
\hline \multirow{2}{*}{$\mathrm{Ja}$} & 688 & 319 & 183 & 120 & $\overline{771}$ & 374 & 1381 \\
\hline & $4,8 \%$ & $7,7 \%$ & $12,5 \%$ & $21,4 \%$ & $25,3 \%$ & $16,3 \%$ & $6,7 \%$ \\
\hline \multirow{2}{*}{ Nein } & 13553 & 3811 & 1276 & 441 & 210 & 1927 & 19291 \\
\hline & $95,2 \%$ & $92,3 \%$ & $87,5 \%$ & $78,6 \%$ & $74,7 \%$ & $83,7 \%$ & $93,3 \%$ \\
\hline > 35 Jahre & $\mathrm{n}=4351$ & $\mathrm{n}=1472$ & $\mathrm{n}=494$ & $\mathrm{n}=178$ & $\mathrm{n}=112$ & $\mathrm{n}=784$ & $\mathrm{n}=6607$ \\
\hline \multirow{2}{*}{$\mathrm{Ja}$} & 310 & 187 & 108 & 49 & 40 & 197 & 699 \\
\hline & $7,1 \%$ & $12,7 \%$ & $21,9 \%$ & $27,5 \%$ & $35,7 \%$ & $25,1 \%$ & $10,5 \%$ \\
\hline \multirow{2}{*}{ Nein } & 4041 & 1285 & 386 & 129 & 72 & 587 & 5913 \\
\hline & $92,9 \%$ & $87,3 \%$ & $78,1 \%$ & $72,5 \%$ & $64,3 \%$ & $74,9 \%$ & $89,5 \%$ \\
\hline
\end{tabular}

\subsubsection{Hypertensive Schwangerschaftserkrankungen}

Die Prävalenz der Gestationshypertonie betrug im Analysezeitraum insgesamt 2,3\% (628 Fälle), wobei unter Berücksichtigung der mütterlichen BMI-Kategorie ein höherer Prozentsatz unter übergewichtigen und adipösen Schwangeren mit 3,0\% bzw. 6,7\% im Vergleich zur Referenzgruppe mit 1,3\% zu finden war (Tab. 15). Unter Einsatz des Chi-Quadrat-Tests wurde deutlich, dass mit ansteigender BMI-Kategorie der Patientinnen von Normalgewicht bis Adipositas Grad III die Frequenz von Gestationshypertonie-Fällen signifikant zunahm. Der Anteil stieg ausgehend von 1,3\% unter normalgewichtigen Schwangeren auf 3,0\% ( $p<0,001)$ unter übergewichtigen Patientinnen an, nahm weiter auf 5,4\% $(\mathrm{p}<0,001)$ und 7,2\% $(\mathrm{p}=0,023)$ unter adipösen Frauen der Schweregrade I und II zu und erreichte ein Maximum von 11,9\% $(\mathrm{p}=0,009)$ unter den Frauen mit einem BMI $\geq 40 \mathrm{~kg} / \mathrm{m}^{2}$. Patientinnen mit einer bereits vor der 
Schwangerschaft diagnostizierten, chronischen Hypertonie $(n=60)$ waren von der Analyse ausgeschlossen.

Tab. 15 Absolute und relative Häufigkeiten einer Gestationshypertonie in Bezug auf maternale BMI-Kategorien

BMI-Kategorien $\left[\mathrm{kg} / \mathrm{m}^{2}\right]$

\begin{tabular}{|c|c|c|c|c|c|c|c|}
\hline $\begin{array}{l}\text { Gestations- } \\
\text { hypertonie }\end{array}$ & $\begin{array}{l}\mathbf{1 8 , 5 - 2 4 , 9} \\
\mathrm{n}=18798\end{array}$ & $\begin{array}{c}\mathbf{2 5 , 0 - 2 9 , 9} \\
\mathrm{n}=5727\end{array}$ & $\begin{array}{c}\mathbf{3 0 , 0 - 3 4 , 9} \\
n=1993\end{array}$ & $\begin{array}{c}\mathbf{3 5 , 0 - 3 9 , 9} \\
n=765\end{array}$ & $\begin{array}{c}\geq 40 \\
n=411\end{array}$ & $\begin{array}{c}\geq \mathbf{3 0} \\
\mathrm{n}=3169\end{array}$ & $\begin{array}{c}\text { Gesamt } \\
\mathrm{N}=27694\end{array}$ \\
\hline \multirow{2}{*}{$\mathrm{Ja}$} & 245 & 172 & 107 & $\overline{c 55}$ & 449 & 211 & 628 \\
\hline & $1,3 \%$ & $3,0 \%$ & $5,4 \%$ & $7,2 \%$ & $11,9 \%$ & $6,7 \%$ & $2,3 \%$ \\
\hline \multirow{2}{*}{ Nein } & 18553 & 5555 & 1886 & 710 & 362 & 2958 & 27066 \\
\hline & $98,7 \%$ & $97,0 \%$ & $94,6 \%$ & $92,8 \%$ & $88,1 \%$ & $93,3 \%$ & $97,7 \%$ \\
\hline
\end{tabular}

Eine Präeklampsie wurde in der Klinik St. Hedwig von 2000 bis 2015 insgesamt bei 554 Frauen diagnostiziert, was einem Anteil von 2,0\% entsprach. Darunter fanden sich 157 Fälle eines HELLP-Syndroms und 16 Fälle einer Eklampsie.

Im Vergleich zu einer Präeklampsie-Rate von 1,5\% unter normalgewichtigen Frauen war der Anteil unter Schwangeren der BMI-Gruppe Übergewicht mit 2,3\% signifikant höher $(\mathrm{p}<0,001)$ ebenso wie unter Frauen der Kategorie Adipositas mit 4,4\% (p<0,001, Chi-Quadrat-Test). Zudem konnte beobachtet werden, dass mit steigendem mütterlichen BMI die relative Häufigkeit einer Präeklampsie zunahm und unter Patientinnen mit Adipositas Grad III ein Maximum von 7,4\% erreichte (Tab. 16; Abb. 11).

Tab. 16 Absolute und relative Häufigkeiten einer Präeklampsie in Bezug auf maternale BMI-Kategorien

BMI-Kategorien $\left[\mathrm{kg} / \mathrm{m}^{2}\right]$

\begin{tabular}{lccccccc}
\cline { 2 - 6 } Präeklampsie & $\begin{array}{cccccc}\mathbf{1 8 , 5 - 2 4 , 9} \\
\mathrm{n}=18809\end{array}$ & $\begin{array}{c}\mathbf{2 5 , 0 - 2 9 , 9} \\
\mathrm{n}=5741\end{array}$ & $\begin{array}{c}\mathbf{3 0 , 0 - 3 4 , 9} \\
\mathrm{n}=2009\end{array}$ & $\begin{array}{c}\mathbf{3 5 , 0 - 3 9 , 9} \\
\mathrm{n}=774\end{array}$ & $\begin{array}{c}\mathbf{2} \text { 40 } \\
\mathrm{n}=421\end{array}$ & $\begin{array}{c}\mathbf{2 3 0} \\
\mathrm{n}=3204\end{array}$ & $\begin{array}{c}\text { Gesamt } \\
\mathrm{N}=27754\end{array}$ \\
\hline \hline \multirow{2}{*}{ Ja } & 279 & 133 & 71 & 40 & 31 & 142 & 554 \\
& $1,5 \%$ & $2,3 \%$ & $3,5 \%$ & $5,2 \%$ & $7,4 \%$ & $4,4 \%$ & $2,0 \%$ \\
\hline \multirow{2}{*}{ Nein } & 18530 & 5608 & 1938 & 734 & 390 & 3062 & 27200 \\
& $98,5 \%$ & $97,7 \%$ & $96,5 \%$ & $94,8 \%$ & $92,6 \%$ & $95,6 \%$ & $98,0 \%$ \\
\hline
\end{tabular}




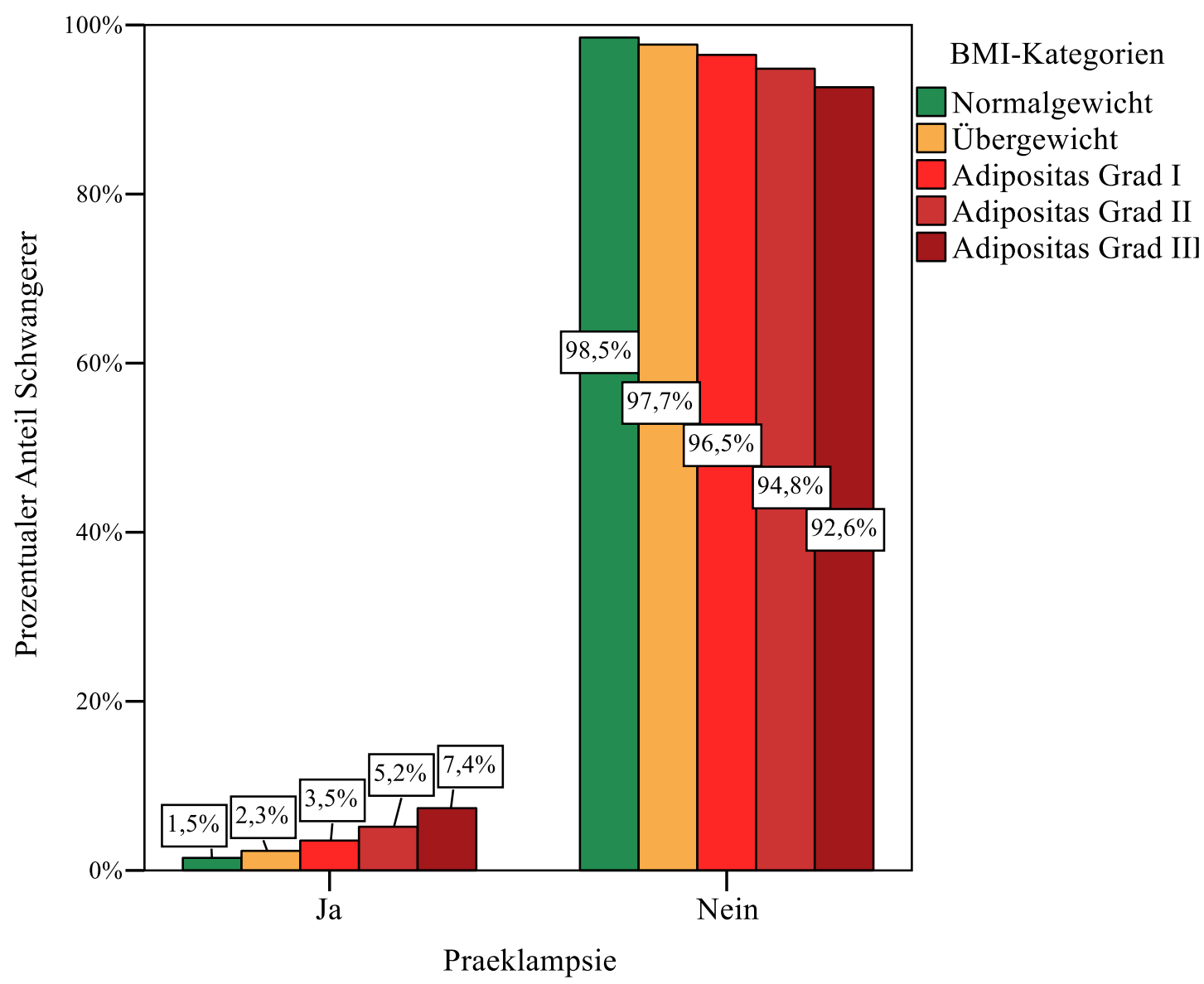

Abb. 11 Relative Häufigkeitsverteilung der Präeklampsie in Bezug auf maternale BMI-Kategorien $(N=27754)$

In Tabellen 17 und 18 sind die schweren Fälle der Präeklampsie in Form eines HELLP-Syndroms $(n=157)$ sowie einer Eklampsie $(n=16)$ einzeln dargestellt.

Innerhalb der Referenzgruppe trat bei 0,6\% der Frauen (113 Fälle) ein HELLP-Syndrom auf, in der BMI-Gruppe Übergewicht waren 23 Schwangere betroffen $(0,4 \%)$ und unter den adipösen Frauen erkrankten $21(0,7 \%)$. Beim Vergleich von übergewichtigen und adipösen Frauen mit der Vergleichsgruppe zeigten sich im Chi-Quadrat-Test keine statistisch signifikanten Unterschiede ( $\mathrm{p}=0,074$ bzw. $\mathrm{p}=0,713)$.

Auch hinsichtlich des Auftretens einer Eklampsie wurden im exakten Test nach Fisher keine signifikanten Unterschiede zwischen normalgewichtigen (9 Fälle), übergewichtigen (4 Fälle) und adipösen Schwangeren (3 Fälle) festgestellt ( $\mathrm{p}=0,517$ bzw. $\mathrm{p}=0,400$ ). 
Tab. 17 Absolute und relative Häufigkeiten eines HELLP-Syndroms in Bezug auf maternale BMI-Kategorien

BMI-Kategorien $\left[\mathrm{kg} / \mathrm{m}^{2}\right]$

\begin{tabular}{|c|c|c|c|c|c|c|c|}
\hline $\begin{array}{l}\text { HELLP- } \\
\text { Syndrom }\end{array}$ & $\begin{array}{r}\mathbf{1 8 , 5}-24,9 \\
n=18809\end{array}$ & $\begin{array}{c}\mathbf{2 5 , 0 - 2 9 , 9} \\
\mathrm{n}=5741\end{array}$ & $\begin{array}{c}\mathbf{3 0 , 0 - 3 4 , 9} \\
\mathrm{n}=2009\end{array}$ & $\begin{array}{c}\mathbf{3 5 , 0 - 3 9 , 9} \\
\mathrm{n}=774\end{array}$ & $\begin{array}{c}\geq \mathbf{4 0} \\
\mathrm{n}=421\end{array}$ & $\begin{array}{c}\geq \mathbf{3 0} \\
\mathrm{n}=3204\end{array}$ & $\begin{array}{c}\text { Gesamt } \\
\mathrm{N}=27754\end{array}$ \\
\hline \multirow{2}{*}{$\mathrm{Ja}$} & 113 & 23 & 10 & 8 & 3 & 21 & 157 \\
\hline & $0,6 \%$ & $0,4 \%$ & $0,5 \%$ & $1,0 \%$ & $0,7 \%$ & $0,7 \%$ & $0,6 \%$ \\
\hline \multirow{2}{*}{ Nein } & 18696 & 5718 & 1999 & 766 & 418 & 3183 & 27597 \\
\hline & $99,4 \%$ & $99,6 \%$ & $99,5 \%$ & $99,0 \%$ & $99,3 \%$ & $99,3 \%$ & $99,4 \%$ \\
\hline
\end{tabular}

Tab. 18 Absolute und relative Häufigkeiten einer Eklampsie in Bezug auf maternale BMI-Kategorien

BMI-Kategorien $\left[\mathrm{kg} / \mathrm{m}^{2}\right]$

\begin{tabular}{lccccccc}
\cline { 2 - 6 } Eklampsii & $\begin{array}{c}\mathbf{1 8 , 5 - 2 4 , 9} \\
\mathrm{n}=18809\end{array}$ & $\begin{array}{c}\mathbf{2 5 , 0 - 2 9 , 9} \\
\mathrm{n}=5741\end{array}$ & $\begin{array}{c}\mathbf{3 0 , 0 - 3 4 , 9} \\
\mathrm{n}=2009\end{array}$ & $\begin{array}{c}\mathbf{3 5 , 0 - 3 9 , 9} \\
\mathrm{n}=774\end{array}$ & $\begin{array}{c}\mathbf{2 4 0} \\
\mathrm{n}=421\end{array}$ & $\begin{array}{c}\mathbf{2} \mathbf{3 0} \\
\mathrm{n}=3204\end{array}$ & $\begin{array}{c}\text { Gesamt } \\
\mathrm{N}=27754\end{array}$ \\
\hline \hline \multirow{2}{*}{ Ja } & 9 & 4 & 2 & 0 & 1 & 3 & 16 \\
& $0,0 \%$ & $0,1 \%$ & $0,1 \%$ & $0,0 \%$ & $0,2 \%$ & $0,1 \%$ & $0,1 \%$ \\
\hline \multirow{2}{*}{ Nein } & 18800 & 5737 & 2007 & 774 & 420 & 3201 & 27738 \\
& $100,0 \%$ & $99,9 \%$ & $100,0 \%$ & $100,0 \%$ & $99,8 \%$ & $99,9 \%$ & $99,9 \%$ \\
\hline
\end{tabular}

In den Subgruppenanalysen bzgl. der Parität der Schwangeren und dem Vorliegen eines Diabetes mellitus blieb der beobachtete signifikante Zusammenhang zwischen dem mütterlichen BMI und der Präeklampsie-Rate jeweils bestehen, was für einen unabhängigen Einfluss der mütterlichen Adipositas von den genannten Faktoren auf das Präeklampsie-Risiko sprach.

In der nach mütterlicher Parität stratifizierten Betrachtung ergab die Auswertung, dass die Präeklampsie innerhalb der Primiparae mit 2,8\% (391 Fälle) signifikant häufiger auftrat ( $<<0,001)$ als unter Pluriparae mit 1,2\% (163 Fälle), wobei die Prävalenz einer Präeklampsie mit dem BMI der Patientinnen sowohl unter Erstgebärenden als auch unter Mehrgebärenden anstieg.

Der Anteil der erkrankten Frauen war unter adipösen Primi- und Pluriparae mit 6,3\% bzw. 3,1\% signifikant höher als unter den Frauen der BMI-Kategorie Normalgewicht mit 2,1\% bzw. 0,7\% (jeweils p<0,001, Chi-Quadrat-Test). In der Gruppe der Primiparae mit einem BMI $\geq 40 \mathrm{~kg} / \mathrm{m}^{2}$ war mit 9,1\% (16 Fälle) die höchste Rate der Erkrankungsfälle zu beobachten (Tab. 19). 
Tab. 19 Absolute und relative Häufigkeiten einer Präeklampsie in Bezug auf maternale BMI-Kategorien, stratifiziert nach Parität

\begin{tabular}{cccccccc} 
& \multicolumn{7}{c}{ BMI-Kategorien $\left[\mathrm{kg} / \mathrm{m}^{2}\right]$} \\
\cline { 2 - 7 } Präeklampsie & $\mathbf{1 8 , 5 - 2 4 , 9}$ & $\mathbf{2 5 , 0 - 2 9 , 9}$ & $\mathbf{3 0 , 0 - 3 4 , 9}$ & $\mathbf{3 5 , 0 - 3 9 , 9}$ & $\mathbf{2 4 0}$ & $\mathbf{2 3 0}$ & Gesamt \\
& $\mathrm{n}=18809$ & $\mathrm{n}=5741$ & $\mathrm{n}=2009$ & $\mathrm{n}=774$ & $\mathrm{n}=421$ & $\mathrm{n}=3204$ & $\mathrm{~N}=27754$ \\
\hline \hline \multirow{2}{*}{ Primipara } & $\mathrm{n}=10007$ & $\mathrm{n}=2567$ & $\mathrm{n}=846$ & $\mathrm{n}=311$ & $\mathrm{n}=175$ & $\mathrm{n}=1332$ & $\mathrm{n}=13906$ \\
\hline \hline \multirow{2}{*}{ Ja } & 215 & 92 & 42 & 26 & 16 & 84 & 391 \\
& $2,1 \%$ & $3,6 \%$ & $5,0 \%$ & $8,4 \%$ & $9,1 \%$ & $6,3 \%$ & $2,8 \%$ \\
\hline \multirow{2}{*}{ Nein } & 9792 & 2475 & 804 & 285 & 159 & 1248 & 13515 \\
& $97,9 \%$ & $96,4 \%$ & $95,0 \%$ & $91,6 \%$ & $90,9 \%$ & $93,7 \%$ & $97,2 \%$ \\
\hline \hline \multirow{2}{*}{ Pluripara } & $\mathrm{n}=8802$ & $\mathrm{n}=3174$ & $\mathrm{n}=1163$ & $\mathrm{n}=463$ & $\mathrm{n}=246$ & $\mathrm{n}=1872$ & $\mathrm{n}=13848$ \\
\hline \hline \multirow{2}{*}{ Ja } & 64 & 41 & 29 & 14 & 15 & 58 & 163 \\
& $0,7 \%$ & $1,3 \%$ & $2,5 \%$ & $3,0 \%$ & $6,1 \%$ & $3,1 \%$ & $1,2 \%$ \\
\hline \multirow{2}{*}{ Nein } & 8738 & 3133 & 1134 & 449 & 231 & 1814 & 13685 \\
& $99,3 \%$ & $98,7 \%$ & $97,5 \%$ & $97,0 \%$ & $93,9 \%$ & $96,9 \%$ & $98,8 \%$ \\
\hline
\end{tabular}

In der Subgruppenanalyse, nach Ausschluss der Frauen mit einer diabetischen Stoffwechsellage (N=25335), sank die Zahl der Präeklampsie-Fälle von insgesamt 554 im Gesamtkollektiv $(2,0 \%)$ auf $486(1,9 \%)$ ab, eine signifikante Assoziation mit dem mütterlichen BMI war jedoch weiterhin zu beobachten (Tab. 20).

Die Präeklampsie trat unter übergewichtigen mit 2,2\% und adipösen Müttern mit 4,3\% häufiger auf als in der normalgewichtigen Vergleichsgruppe mit 1,5\%. Die bivariate Analyse erfolgte auch hier über den Chi-Quadrat-Test und zeigte mit jeweils $p<0,001$ ein statistisch signifikantes Ergebnis.

Tab. 20 Absolute und relative Häufigkeiten einer Präeklampsie in Bezug auf maternale BMI-Kategorien in der Subgruppe stoffwechselgesunder Frauen

BMI-Kategorien $\left[\mathrm{kg} / \mathrm{m}^{2}\right]$

\begin{tabular}{|c|c|c|c|c|c|c|c|}
\hline osie & $\begin{array}{r}\mathbf{1 8 , 5 - 2 4 , 9} \\
\mathrm{n}=17689\end{array}$ & $\begin{array}{c}\mathbf{2 5 , 0 - 2 9 , 9} \\
\mathrm{n}=5124\end{array}$ & $\begin{array}{c}\mathbf{3 0 , 0 - 3 4 , 9} \\
\mathrm{n}=1668\end{array}$ & $\begin{array}{c}\mathbf{3 5 , 0 - 3 9 , 9} \\
\mathrm{n}=572\end{array}$ & $\begin{array}{c}\geq \mathbf{4 0} \\
\mathrm{n}=282\end{array}$ & $\begin{array}{c}\geq \mathbf{3 0} \\
\mathrm{n}=2522\end{array}$ & $\begin{array}{c}\text { Gesamt } \\
\mathrm{N}=25335\end{array}$ \\
\hline \multirow{2}{*}{$\mathrm{Ja}$} & 263 & 114 & 58 & 32 & 19 & 109 & 486 \\
\hline & $1,5 \%$ & $2,2 \%$ & $3,5 \%$ & $5,6 \%$ & $6,7 \%$ & $4,3 \%$ & $1,9 \%$ \\
\hline \multirow[t]{2}{*}{ Nein } & $\begin{array}{l}17426 \\
085 \%\end{array}$ & $\begin{array}{l}5010 \\
078 \%\end{array}$ & $\begin{array}{l}1610 \\
065 \%\end{array}$ & 540 & 263 & $\begin{array}{c}2413 \\
057 \%\end{array}$ & $\begin{array}{l}24849 \\
081 \%\end{array}$ \\
\hline & $98,5 \%$ & $97,8 \%$ & $96,5 \%$ & $94,4 \%$ & $93,3 \%$ & $95,7 \%$ & $98,1 \%$ \\
\hline
\end{tabular}




\subsubsection{Nikotinabusus}

Insgesamt gaben 396 Patientinnen (1,4\%) an, während der Schwangerschaft geraucht zu haben. Im Vergleich zu den Frauen der Referenzgruppe mit 1,2\% rauchten übergewichtige ( $<<0,001)$ und adipöse Mütter ( $\mathrm{p}=0,005)$ mit jeweils 1,8\% signifikant häufiger während der Schwangerschaft (Chi-Quadrat-Test). In der BMI-Kategorie Adipositas Grad III war der prozentuale Anteil von Frauen mit einem angegebenen Nikotinabusus während der Schwangerschaft mit 3,1\% am höchsten (Tab. 21).

Tab. 21 Absolute und relative Häufigkeiten eines Nikotinabusus während der Schwangerschaft in Bezug auf maternale BMI-Kategorien

BMI-Kategorien $\left[\mathrm{kg} / \mathrm{m}^{2}\right]$

\begin{tabular}{|c|c|c|c|c|c|c|c|}
\hline $\begin{array}{l}\text { Nikotin- } \\
\text { abusus }\end{array}$ & $\begin{array}{c}\mathbf{1 8 , 5 - 2 4 , 9} \\
n=18809\end{array}$ & $\begin{array}{c}\mathbf{2 5 , 0 - 2 9 , 9} \\
n=5741\end{array}$ & $\begin{array}{c}\mathbf{3 0 , 0 - 3 4 , 9} \\
n=2009\end{array}$ & $\begin{array}{c}\mathbf{3 5 , 0 - 3 9 , 9} \\
n=774\end{array}$ & $\begin{array}{c}\geq \mathbf{4 0} \\
\mathrm{n}=421\end{array}$ & $\begin{array}{c}\geq \mathbf{3 0} \\
n=3204\end{array}$ & $\begin{array}{c}\text { Gesamt } \\
\mathrm{N}=27754\end{array}$ \\
\hline \multirow{2}{*}{$\mathrm{Ja}$} & 231 & 106 & 34 & 12 & 13 & "59 & 396 \\
\hline & $1,2 \%$ & $1,8 \%$ & $1,7 \%$ & $1,6 \%$ & $3,1 \%$ & $1,8 \%$ & $1,4 \%$ \\
\hline Nein & $\begin{array}{l}18578 \\
98.8 \%\end{array}$ & $\begin{array}{c}5635 \\
98,2 \%\end{array}$ & $\begin{array}{c}1975 \\
98,3 \%\end{array}$ & $\begin{array}{c}762 \\
98,4 \%\end{array}$ & $\begin{array}{c}408 \\
96,9 \%\end{array}$ & $\begin{array}{c}3145 \\
98,2 \%\end{array}$ & $\begin{array}{l}27358 \\
98,6 \%\end{array}$ \\
\hline
\end{tabular}

\subsubsection{Psychische Belastung}

Eine besondere psychische Belastung während der Schwangerschaft wurde in 86 Fällen dokumentiert $(0,3 \%)$. Sie trat in 57 Fällen bei normalgewichtigen $(0,3 \%)$, in 23 Fällen bei übergewichtigen $(0,4 \%)$ und in 6 Fällen $(0,2 \%)$ bei adipösen Patientinnen auf. Im Chi-Quadrat-Test konnten keine statistisch signifikanten Unterschiede der Häufigkeit einer psychischen Belastung der Schwangeren bei übergewichtigen $(p=0,256)$ und adipösen Frauen $(p=0,257)$ im Vergleich zum Referenzkollektiv der normalgewichtigen Mütter festgestellt werden (Tab. 22).

Tab. 22 Absolute und relative Häufigkeiten einer besonderen psychischen Belastung während der Schwangerschaft in Bezug auf maternale BMI-Kategorien

BMI-Kategorien $\left[\mathrm{kg} / \mathrm{m}^{2}\right]$

\begin{tabular}{|c|c|c|c|c|c|c|c|}
\hline $\begin{array}{l}\text { Psychische } \\
\text { Belastung }\end{array}$ & $\begin{array}{c}\mathbf{1 8 , 5}-24,9 \\
n=18809\end{array}$ & $\begin{array}{c}\mathbf{2 5 , 0 - 2 9 , 9} \\
n=5741\end{array}$ & $\begin{array}{c}\mathbf{3 0 , 0 - 3 4 , 9} \\
\mathrm{n}=2009\end{array}$ & $\begin{array}{c}\mathbf{3 5 , 0 - 3 9 , 9} \\
n=774\end{array}$ & $\begin{array}{c}\geq \mathbf{4 0} \\
n=421\end{array}$ & $\begin{array}{c}\geq \mathbf{3 0} \\
n=3204\end{array}$ & $\begin{array}{c}\text { Gesamt } \\
\mathrm{N}=27754\end{array}$ \\
\hline \multirow{2}{*}{$\mathrm{Ja}$} & $\overline{57}$ & 23 & 3 & $\overline{3}$ & $\overline{00}$ & $\overline{66}$ & 86 \\
\hline & $0,3 \%$ & $0,4 \%$ & $0,1 \%$ & $0,4 \%$ & $0,0 \%$ & $0,2 \%$ & $0,3 \%$ \\
\hline \multirow{2}{*}{ Nein } & 18752 & 5718 & 2006 & 771 & 421 & 3198 & 27668 \\
\hline & $99,7 \%$ & $99,6 \%$ & $99,9 \%$ & $99,6 \%$ & $100,0 \%$ & $99,9 \%$ & $99,7 \%$ \\
\hline
\end{tabular}




\subsubsection{Harnwegsinfekt}

Die Prävalenz eines Harnwegsinfekts in der Schwangerschaft betrug im Untersuchungszeitraum mit 101 dokumentierten Fällen insgesamt 0,4\% (Tab. 23). Bei übergewichtigen und adipösen Frauen kam es mit 0,5\% (26 Fälle) bzw. 0,4\% (14 Fälle) prozentual etwas häufiger zu einem Harnwegsinfekt als bei normalgewichtigen Schwangeren mit 0,3\% (61 Fälle). Die Unterschiede zwischen den BMI-Gruppen wurden mithilfe des Chi-Quadrat-Tests jedoch als nicht statistisch signifikant belegt ( $\mathrm{p}=0,151$ bzw. $\mathrm{p}=0,312)$.

Tab. 23 Absolute und relative Häufigkeiten eines Harnwegsinfekts in Bezug auf maternale BMI-Kategorien

BMI-Kategorien $\left[\mathrm{kg} / \mathrm{m}^{2}\right]$

\begin{tabular}{|c|c|c|c|c|c|c|c|}
\hline $\begin{array}{l}\text { Harnwegs- } \\
\text { infekt }\end{array}$ & $\begin{array}{c}\mathbf{1 8 , 5 - 2 4 , 9} \\
\mathrm{n}=18809\end{array}$ & $\begin{array}{c}\mathbf{2 5 , 0 - 2 9 , 9} \\
\mathrm{n}=5741\end{array}$ & $\begin{array}{c}\mathbf{3 0 , 0 - 3 4 , 9} \\
\mathrm{n}=2009\end{array}$ & $\begin{array}{c}\mathbf{3 5 , 0 - 3 9 , 9} \\
n=774\end{array}$ & $\begin{array}{c}\geq \mathbf{4 0} \\
\mathrm{n}=421\end{array}$ & $\begin{array}{c}\geq \mathbf{3 0} \\
n=3204\end{array}$ & $\begin{array}{c}\text { Gesamt } \\
\mathrm{N}=27754\end{array}$ \\
\hline \multirow{2}{*}{$\mathrm{Ja}$} & "61 & 26 & "9 & $\overline{2}$ & 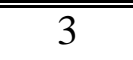 & $\overline{14}$ & "101 \\
\hline & $0,3 \%$ & $0,5 \%$ & $0,4 \%$ & $0,3 \%$ & $0,7 \%$ & $0,4 \%$ & $0,4 \%$ \\
\hline Nein & $\begin{array}{l}18748 \\
997 \%\end{array}$ & $\begin{array}{c}5715 \\
995 \%\end{array}$ & $\begin{array}{l}2000 \\
996 \%\end{array}$ & $\begin{array}{c}772 \\
997 \%\end{array}$ & $\begin{array}{c}418 \\
093 \%\end{array}$ & $\begin{array}{l}3190 \\
996 \%\end{array}$ & $\begin{array}{l}27653 \\
996 \%\end{array}$ \\
\hline
\end{tabular}

\subsubsection{Fetale Wachstumsrestriktion}

Insgesamt waren im Zeitraum der Analyse 798 Schwangerschaften (2,9\%) von einer fetalen Wachstumsrestriktion betroffen (Tab. 24). Davon gehörten 530 Frauen der Kategorie Normalgewicht an (2,8\%), 165 Frauen der Kategorie Übergewicht (2,9\%) und 103 Frauen der BMIGruppe Adipositas (3,2\%).

Der Anteil von wachstumsretardierten Feten war somit unter übergewichtigen und adipösen Schwangeren prozentual etwas höher als unter normalgewichtigen Frauen. Die Unterschiede zwischen den BMI-Gruppen zeigten sich im Chi-Quadrat-Test jedoch als nicht statistisch signifikant ( $\mathrm{p}=0,822$ bzw. $\mathrm{p}=0,214)$.

Tab. 24 Absolute und relative Häufigkeiten eines IUGR in Bezug auf maternale BMI-Kategorien

BMI-Kategorien $\left[\mathrm{kg} / \mathrm{m}^{2}\right]$

\begin{tabular}{|c|c|c|c|c|c|c|c|}
\hline $\begin{array}{l}\text { Wachstums- } \\
\text { restriktion }\end{array}$ & $\begin{array}{c}\mathbf{1 8 , 5 - 2 4 , 9} \\
\mathrm{n}=18809\end{array}$ & $\begin{array}{c}\mathbf{2 5 , 0 - 2 9 , 9} \\
n=5741\end{array}$ & $\begin{array}{c}\mathbf{3 0 , 0 - 3 4 , 9} \\
\mathrm{n}=2009\end{array}$ & $\begin{array}{c}\text { 35,0-39,9 } \\
n=774\end{array}$ & $\begin{array}{c}\geq \mathbf{4 0} \\
\mathrm{n}=421\end{array}$ & $\begin{array}{c}\geq \mathbf{3 0} \\
n=3204\end{array}$ & $\begin{array}{c}\text { Gesamt } \\
\mathrm{N}=27754\end{array}$ \\
\hline \multirow{2}{*}{$\mathrm{Ja}$} & ב530 & 165 & 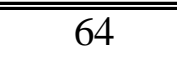 & 26 & $\bar{~} 13$ & 103 & 798 \\
\hline & $2,8 \%$ & $2,9 \%$ & $3,2 \%$ & $3,4 \%$ & $3,1 \%$ & $3,2 \%$ & $2,9 \%$ \\
\hline Nein & $\begin{array}{l}18279 \\
072 \%\end{array}$ & $\begin{array}{l}5576 \\
071 \%\end{array}$ & $\begin{array}{l}1945 \\
968 \%\end{array}$ & $\begin{array}{c}748 \\
966 \%\end{array}$ & $\begin{array}{c}408 \\
060 \%\end{array}$ & $\begin{array}{c}3101 \\
968 \%\end{array}$ & $\begin{array}{l}26956 \\
071 \%\end{array}$ \\
\hline
\end{tabular}




\subsubsection{Vorzeitige Wehentätigkeit}

Bei der Analyse der Häufigkeit der vorzeitigen Wehentätigkeit bestätigte sich unter Anwendung des Chi-Quadrat-Tests, dass dieses Ereignis im Vergleich zur Gruppe der normalgewichtigen Schwangeren mit 4,7\% bei übergewichtigen und adipösen Frauen mit 3,5\% bzw. 3,1\% signifikant seltener auftrat (jeweils p<0,001). Insgesamt kam es im Analysezeitraum bei 1190 Patientinnen (4,3\%) zu vorzeitigen, zervixwirksamen Wehen (Tab. 25).

Tab. 25 Absolute und relative Häufigkeiten der vorzeitigen Wehentätigkeit in Bezug auf maternale BMI-Kategorien

BMI-Kategorien $\left[\mathrm{kg} / \mathrm{m}^{2}\right]$

\begin{tabular}{|c|c|c|c|c|c|c|c|}
\hline $\begin{array}{l}\text { Vorzeitige } \\
\text { Wehen }\end{array}$ & $\begin{array}{c}\mathbf{1 8 , 5 - 2 4 , 9} \\
\mathrm{n}=18809\end{array}$ & $\begin{array}{c}\mathbf{2 5 , 0 - 2 9 , 9} \\
n=5741\end{array}$ & $\begin{array}{c}\mathbf{3 0 , 0 - 3 4 , 9} \\
\mathrm{n}=2009\end{array}$ & $\begin{array}{c}35,0-39,9 \\
n=774\end{array}$ & $\begin{array}{c}\geq 40 \\
n=421\end{array}$ & $\begin{array}{c}\geq \mathbf{3 0} \\
n=3204\end{array}$ & $\begin{array}{c}\text { Gesamt } \\
\mathrm{N}=27754\end{array}$ \\
\hline \multirow{2}{*}{$\mathrm{Ja}$} & 890 & 202 & 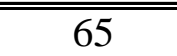 & 21 & 12 & 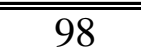 & 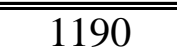 \\
\hline & $4,7 \%$ & $3,5 \%$ & $3,2 \%$ & $2,7 \%$ & $2,9 \%$ & $3,1 \%$ & $4,3 \%$ \\
\hline \multirow{2}{*}{ Nein } & 17919 & 5539 & 1944 & 753 & 409 & 3106 & 26564 \\
\hline & $95,3 \%$ & $96,5 \%$ & $96,8 \%$ & $97,3 \%$ & $97,1 \%$ & $96,9 \%$ & $95,7 \%$ \\
\hline
\end{tabular}




\subsection{Geburtsverlauf}

\subsubsection{Vorzeitiger Blasensprung}

Ein vorzeitiger Blasensprung war insgesamt bei 29,3\% der Geburten (8137 Fälle) zu finden. Bezogen auf die mütterliche BMI-Kategorie war der Anteil unter übergewichtigen mit 29,5\% $(\mathrm{p}=0,569)$ und adipösen Schwangeren mit 29,9\% ( $\mathrm{p}=0,389)$ prozentual geringfügig höher als unter normalgewichtigen Frauen mit 29,2\%, wobei sich die Unterschiede im durchgeführten Chi-Quadrat-Test als nicht statistisch signifikant erwiesen (Tab. 26).

Tab. 26 Absolute und relative Häufigkeiten eines vorzeitigen Blasensprungs in Bezug auf maternale BMI-Kategorien

BMI-Kategorien $\left[\mathrm{kg} / \mathrm{m}^{2}\right]$

\begin{tabular}{|c|c|c|c|c|c|c|c|}
\hline $\begin{array}{l}\text { Vorzeitiger } \\
\text { Blasensprung }\end{array}$ & $\begin{array}{c}\mathbf{1 8 , 5 - 2 4 , 9} \\
\mathrm{n}=18809\end{array}$ & $\begin{array}{c}\mathbf{2 5 , 0 - 2 9 , 9} \\
n=5741\end{array}$ & $\begin{array}{c}\mathbf{3 0 , 0 - 3 4 , 9} \\
n=2009\end{array}$ & $\begin{array}{c}\mathbf{3 5 , 0 - 3 9 , 9} \\
n=774\end{array}$ & $\begin{array}{c}\geq \mathbf{4 0} \\
n=421\end{array}$ & $\begin{array}{c}\geq \mathbf{3 0} \\
n=3204\end{array}$ & $\begin{array}{c}\text { Gesamt } \\
\mathrm{N}=27754\end{array}$ \\
\hline \multirow{2}{*}{$\mathrm{Ja}$} & $\bar{~} 5483$ & 1696 & 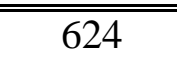 & 231 & 103 & 958 & 8137 \\
\hline & $29,2 \%$ & $29,5 \%$ & $31,1 \%$ & $29,8 \%$ & $24,5 \%$ & $29,9 \%$ & $29,3 \%$ \\
\hline Nein & $\begin{array}{l}13326 \\
70807\end{array}$ & $\begin{array}{c}4045 \\
705 \%\end{array}$ & $\begin{array}{l}1385 \\
680 \%\end{array}$ & $\begin{array}{c}543 \\
7020 \%\end{array}$ & $\begin{array}{c}318 \\
755 \%\end{array}$ & 2246 & $\begin{array}{l}19617 \\
707 \%\end{array}$ \\
\hline
\end{tabular}

\subsubsection{Geburtsmodus und Indikationen zur Sectio caesarea}

Laut den Daten der Klinik St. Hedwig wurden im Untersuchungszeitraum insgesamt 23,5\% der Entbindungen (6532 Fälle) per Sectio caesarea durchgeführt, wobei diese in 11,6\% der Fälle primär und in 11,9\% der Fälle sekundär erfolgte. Sowohl der primäre als auch der sekundäre Kaiserschnitt wurde dabei unter übergewichtigen mit 12,8\% bzw. 12,9\% und unter adipösen Frauen mit 18,2\% bzw. 15,5\% häufiger durchgeführt als unter den normalgewichtigen Patientinnen mit 10,2\% bzw. 11,0\%. Die Unterschiede zwischen den Gruppen stellten sich unter Anwendung des Chi-Quadrat-Tests mit jeweils $\mathrm{p}<0,001$ als statistisch signifikant heraus. Die Rate der primär und sekundär ausgeführten abdominellen Entbindungen stieg mit zunehmendem BMI der Schwangeren an und erreichte unter den Frauen mit einem BMI $\geq 40 \mathrm{~kg} / \mathrm{m}^{2}$ die Höchstwerte von 22,9\% bzw. 17,1\% (Tab. 27; Abb. 12).

Der Großteil der Schwangeren entband mit insgesamt 69,1\% spontan vaginal aus Schädellage (SL), wobei unter Berücksichtigung der mütterlichen BMI-Kategorie ein signifikant höherer Prozentsatz unter normalgewichtigen Frauen mit 70,8\% im Vergleich zu übergewichtigen und adipösen Patientinnen mit 68,0\% bzw. 61,2\% zu finden war (jeweils p<0,001, Chi-QuadratTest). 
Dabei konnte ausgehend von der Referenzgruppe eine Abnahme der Häufigkeit dieses Entbindungsmodus mit steigendem BMI der Mutter festgestellt werden. Die niedrigste Rate der spontan vaginalen Entbindungen aus Schädellage war unter den Frauen der Kategorie Adipositas Grad III mit einer Häufigkeit von 54,8\% zu beobachten (Tab. 27; Abb. 12).

Die Kinder von adipösen Frauen wurden im Vergleich zu denen normalgewichtiger Frauen zudem signifikant seltener vaginal-operativ $(4,4 \%$ gegenüber $6,9 \%$; $<<0,001)$ und in vaginaler Beckenendlage (BEL) (0,7\% gegenüber 1,2\%; $\mathrm{p}=0,009)$ zur Welt gebracht (Tab. 27; Abb. 12). In diesem Punkt muss jedoch hinzugefügt werden, dass der Anteil der Schwangerschaften mit kindlicher Beckenendlage insgesamt unter normalgewichtigen Frauen mit 7,2\% (1356 Fälle) signifikant höher war als unter adipösen Frauen mit 6,2\% (198 Fälle, p=0,035) (Tab. 28).

Tab. 27 Absolute und relative Häufigkeiten der verschiedenen Geburtsmodi in Bezug auf maternale BMI-Kategorien

BMI-Kategorien $\left[\mathrm{kg} / \mathrm{m}^{2}\right]$

\begin{tabular}{|c|c|c|c|c|c|c|c|}
\hline \multirow[b]{2}{*}{ Geburtsmodus } & & \multirow[b]{2}{*}{$\begin{array}{c}\text { Gesamt } \\
\mathrm{N}=27747\end{array}$} \\
\hline & $\begin{array}{c}\mathbf{1 8 , 5 - 2 4 , 9} \\
n=18806\end{array}$ & $\begin{array}{c}\mathbf{2 5 , 0 - 2 9 , 9} \\
\mathrm{n}=5738\end{array}$ & $\begin{array}{c}\mathbf{3 0 , 0 - 3 4 , 9} \\
\mathrm{n}=2009\end{array}$ & $\begin{array}{c}\mathbf{3 5 , 0 - 3 9 , 9} \\
\mathrm{n}=774\end{array}$ & $\begin{array}{c}\geq \mathbf{4 0} \\
\mathrm{n}=420\end{array}$ & $\begin{array}{c}\geq \mathbf{3 0} \\
\mathrm{n}=3203\end{array}$ & \\
\hline \multirow{2}{*}{$\begin{array}{l}\text { Spontan vaginal } \\
\text { aus SL }\end{array}$} & 13312 & 3899 & 1277 & 454 & 230 & 1961 & 19172 \\
\hline & $70,8 \%$ & $68,0 \%$ & $63,6 \%$ & $58,7 \%$ & $54,8 \%$ & $61,2 \%$ & $69,1 \%$ \\
\hline \multirow{2}{*}{ Vaginal-operativ } & 1295 & 320 & 91 & 31 & 19 & 141 & 1756 \\
\hline & $6,9 \%$ & $5,6 \%$ & $4,5 \%$ & $4,0 \%$ & $4,5 \%$ & $4,4 \%$ & $6,3 \%$ \\
\hline \multirow{2}{*}{ Vaginale BEL } & 222 & 44 & 12 & 6 & 3 & 21 & 287 \\
\hline & $1,2 \%$ & $0,8 \%$ & $0,6 \%$ & $0,8 \%$ & $0,7 \%$ & $0,7 \%$ & $1,0 \%$ \\
\hline \multirow{2}{*}{ Primäre Sectio } & 1910 & 737 & 328 & 158 & 96 & 582 & 3229 \\
\hline & $10,2 \%$ & $12,8 \%$ & $16,3 \%$ & $20,4 \%$ & $22,9 \%$ & $18,2 \%$ & $11,6 \%$ \\
\hline \multirow{2}{*}{ Sekundäre Sectio } & 2067 & 738 & 301 & 125 & 72 & 498 & 3303 \\
\hline & $11,0 \%$ & $12,9 \%$ & $15,0 \%$ & $16,1 \%$ & $17,1 \%$ & $15,5 \%$ & $11,9 \%$ \\
\hline \multirow{2}{*}{ Sectio gesamt } & 3977 & 141475 & "629 & 283 & 168 & 101080 & 6532 \\
\hline & $21,1 \%$ & $25,7 \%$ & $31,3 \%$ & $36,6 \%$ & $40,0 \%$ & $33,7 \%$ & $23,5 \%$ \\
\hline
\end{tabular}

Tab. 28 Absolute und relative Häufigkeiten einer Beckenendlage gesamt in Bezug auf maternale BMI-Kategorien

BMI-Kategorien $\left[\mathrm{kg} / \mathrm{m}^{2}\right]$

\begin{tabular}{lccccccc} 
Becken- & $\mathbf{1 8 , 5 - 2 4 , 9}$ & $\mathbf{2 5 , 0 - 2 9 , 9}$ & $\mathbf{3 0 , 0 - 3 4 , 9}$ & $\mathbf{3 5 , 0 - 3 9 , 9}$ & $\mathbf{2 4 0}$ & $\mathbf{2 3 0}$ & Gesamt \\
endlage & $\mathrm{n}=18809$ & $\mathrm{n}=5741$ & $\mathrm{n}=2009$ & $\mathrm{n}=774$ & $\mathrm{n}=421$ & $\mathrm{n}=3204$ & $\mathrm{~N}=27754$ \\
\hline \hline \multirow{2}{*}{ Ja } & 1356 & 339 & 120 & 51 & 27 & 198 & 1893 \\
& $7,2 \%$ & $5,9 \%$ & $6,0 \%$ & $6,6 \%$ & $6,4 \%$ & $6,2 \%$ & $6,8 \%$ \\
\hline \multirow{2}{*}{ Nein } & 17453 & 5402 & 1889 & 723 & 394 & 3006 & 25861 \\
& $92,8 \%$ & $94,1 \%$ & $94,0 \%$ & $93,4 \%$ & $93,6 \%$ & $93,8 \%$ & $93,2 \%$ \\
\hline
\end{tabular}




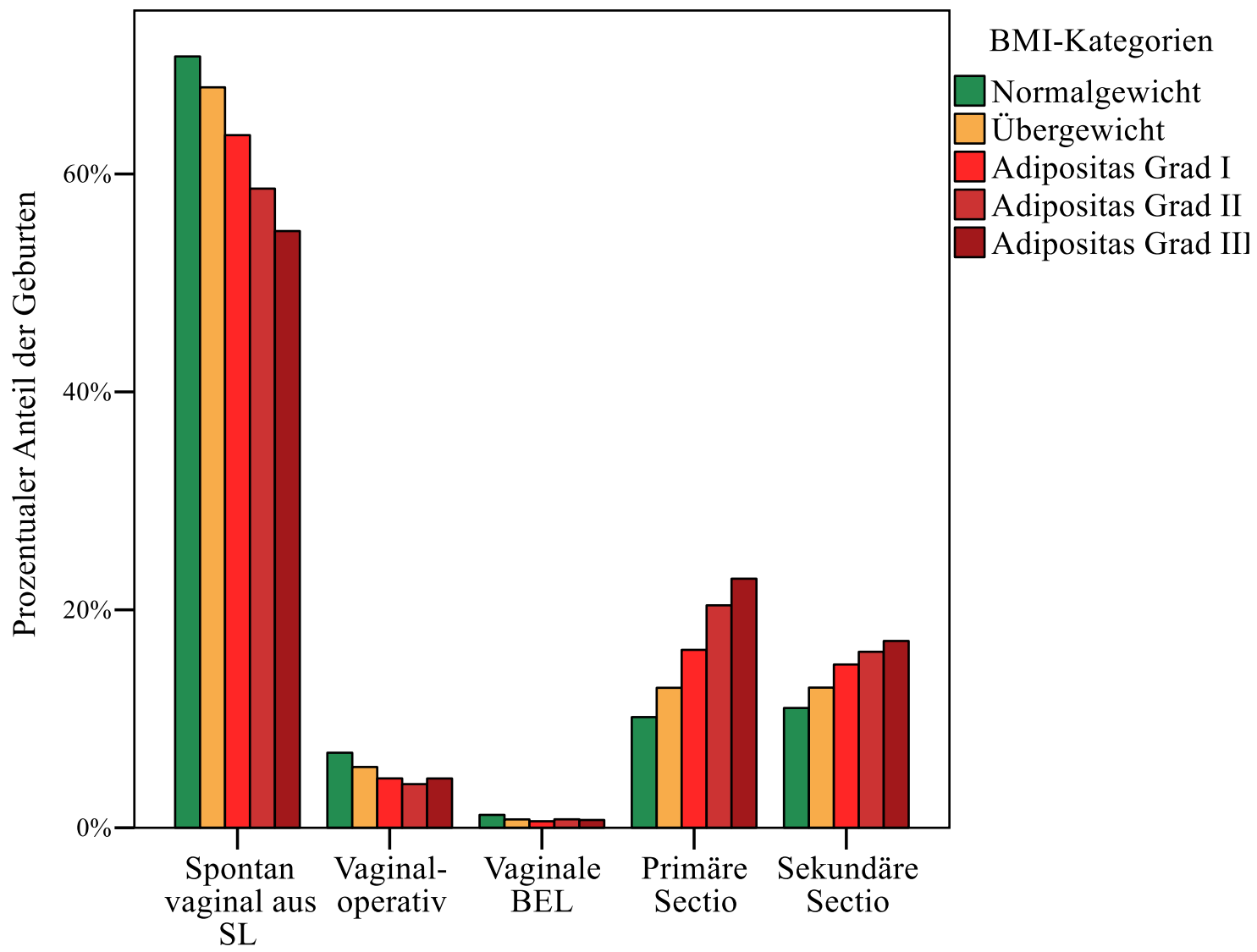

Geburtsmodus

Abb. 12 Relative Häufigkeitsverteilung der verschiedenen Geburtsmodi in Bezug auf maternale BMI-Kategorien $(N=27747)$

Der Einfluss eines hohen mütterlichen BMI auf das Sectio-Risiko stellte sich durch die Analyse in Subgruppen als unabhängig von der Parität, dem Vorliegen einer Geburtseinleitung sowie von adipositas-assoziierten Komorbiditäten der Schwangeren heraus (Tab. 29 bis 31).

In der nach mütterlicher Parität stratifizierten Subgruppenanalyse wurde deutlich, dass 25,6\% der Frauen innerhalb der Gruppe der Primiparae sectioniert wurden, was signifikant höher war im Vergleich zur Kaiserschnitt-Rate unter Pluriparae mit 21,4\% (Tab. 29). In der Gruppe der Erstgebärenden wurde sowohl die primäre als auch die sekundäre Sectio caesarea mit 13,8\% bzw. 22,1\% unter adipösen Schwangeren signifikant häufiger durchgeführt als unter normalgewichtigen Frauen mit 9,3\% bzw. 14,3\%. Auch unter Pluriparae wurde der primäre und sekundäre Kaiserschnitt mit 21,3\% bzw. 10,9\% unter Frauen mit einem BMI $\geq 30 \mathrm{~kg} / \mathrm{m}^{2}$ signifikant häufiger als Entbindungsmodus angewandt als unter den Frauen der Referenzgruppe mit 11,1\% bzw. 7,2\%. Die bivariate Analyse erfolgte in allen Fällen über den Chi-Quadrat-Test und bestätigte mit jeweils $\mathrm{p}<0,001$ die statistische Signifikanz. 
Tab. 29 Absolute und relative Häufigkeiten der Sectio caesarea in Bezug auf maternale BMI-Kategorien, stratifiziert nach Parität

BMI-Kategorien $\left[\mathrm{kg} / \mathrm{m}^{2}\right]$

\begin{tabular}{lccccccc}
\cline { 2 - 6 } $\begin{array}{l}\text { Geburts- } \\
\text { modus }\end{array}$ & $\mathbf{1 8 , 5 - 2 4 , 9}$ & $\mathbf{2 5 , 0 - 2 9 , 9}$ & $\mathbf{3 0 , 0 - 3 4 , 9}$ & $\mathbf{3 5 , 0 - 3 9 , 9}$ & $\mathbf{2} \mathbf{4 0}$ & $\mathbf{2} \mathbf{3 0}$ & Gesamt \\
\hline \hline Primipara & $\mathrm{n}=18006$ & $\mathrm{n}=5738$ & $\mathrm{n}=2009$ & $\mathrm{n}=774$ & $\mathrm{n}=420$ & $\mathrm{n}=3203$ & $\mathrm{~N}=27747$ \\
\hline \hline Primäre & 934 & 273 & 109 & 44 & 31 & 184 & 1391 \\
Sectio & $9,3 \%$ & $10,6 \%$ & $12,9 \%$ & $14,1 \%$ & $17,7 \%$ & $13,8 \%$ & $10,0 \%$ \\
\hline Sekundäre & 1429 & 449 & 176 & 76 & 42 & 294 & 2172 \\
Sectio & $14,3 \%$ & $17,5 \%$ & $20,8 \%$ & $24,4 \%$ & $24,0 \%$ & $22,1 \%$ & $15,6 \%$ \\
\hline Sectio & 2363 & 722 & 285 & 120 & 73 & 478 & 3563 \\
gesamt & $23,6 \%$ & $28,1 \%$ & $33,7 \%$ & $38,6 \%$ & $41,7 \%$ & $35,9 \%$ & $25,6 \%$ \\
\hline Pluripara & $\mathrm{n}=8800$ & $\mathrm{n}=3172$ & $\mathrm{n}=1163$ & $\mathrm{n}=463$ & $\mathrm{n}=245$ & $\mathrm{n}=1871$ & $\mathrm{n}=13843$ \\
\hline \hline Primäre & 976 & 464 & 219 & 114 & 65 & 398 & 1838 \\
Sectio & $11,1 \%$ & $14,6 \%$ & $18,8 \%$ & $24,6 \%$ & $26,5 \%$ & $21,3 \%$ & $13,3 \%$ \\
\hline Sekundäre & 638 & 289 & 125 & 49 & 30 & 204 & 1131 \\
Sectio & $7,2 \%$ & $9,1 \%$ & $10,7 \%$ & $10,6 \%$ & $12,2 \%$ & $10,9 \%$ & $8,2 \%$ \\
\hline Sectio & 1614 & 753 & 344 & 163 & 95 & 602 & 2969 \\
gesamt & $18,3 \%$ & $23,7 \%$ & $29,6 \%$ & $35,2 \%$ & $38,8 \%$ & $32,2 \%$ & $21,4 \%$ \\
\hline
\end{tabular}

Bei der Auswertung der Sectio-Rate in Subgruppen, stratifiziert nach dem Vorliegen einer Geburtseinleitung, wurde zunächst deutlich, dass es unter eingeleiteten Geburten mit insgesamt 17,2\% signifikant häufiger ( $\mathrm{p}<0,001)$ zu einer sekundär durchgeführten abdominellen Entbindung kam als unter Geburten mit spontanem Beginn mit 10,3\% (Tab. 30). Sowohl bei eingeleiteten $(\mathrm{p}<0,001)$ als auch bei nicht-eingeleiteten Geburten $(\mathrm{p}=0,002)$ wurden adipöse Frauen mit 22,4\% bzw. 12,1\% signifikant häufiger sekundär sectioniert als normalgewichtige Gebärende mit 15,4\% bzw. 9,9\%. Die Unterschiede zwischen den BMI-Gruppen stellten sich jeweils mithilfe des Chi-Quadrat-Tests als statistisch signifikant heraus. 
Tab. 30 Häufigkeit der sekundären Sectio in Bezug auf mütterliche BMI-Kategorien, stratifiziert nach dem Vorliegen einer Geburtseinleitung

BMI-Kategorien $\left[\mathrm{kg} / \mathrm{m}^{2}\right]$

\begin{tabular}{|c|c|c|c|c|c|c|c|}
\hline $\begin{array}{l}\text { Geburts- } \\
\text { einleitung }\end{array}$ & $\begin{array}{r}\mathbf{1 8 , 5}-\mathbf{2 4 , 9} \\
n=18806\end{array}$ & $\begin{array}{c}\mathbf{2 5 , 0 - 2 9 , 9} \\
n=5738\end{array}$ & $\begin{array}{c}\mathbf{3 0 , 0 - 3 4 , 9} \\
n=2009\end{array}$ & $\begin{array}{c}\mathbf{3 5 , 0 - 3 9 , 9} \\
n=774\end{array}$ & $\begin{array}{c}\geq \mathbf{4 0} \\
n=420\end{array}$ & $\begin{array}{c}\geq \mathbf{3 0} \\
n=3203\end{array}$ & $\begin{array}{c}\text { Gesamt } \\
\mathrm{N}=27747\end{array}$ \\
\hline $\mathrm{Ja}$ & $\mathrm{n}=3840$ & $\mathrm{n}=1483$ & $\mathrm{n}=620$ & $\mathrm{n}=287$ & $\mathrm{n}=174$ & $\mathrm{n}=1081$ & $\mathrm{n}=6404$ \\
\hline Sekundäre & 591 & 269 & 123 & 65 & 54 & 242 & 1102 \\
\hline Sectio & $15,4 \%$ & $18,1 \%$ & $19,8 \%$ & $22,6 \%$ & $31,0 \%$ & $22,4 \%$ & $17,2 \%$ \\
\hline Nein & $n=14966$ & $\mathrm{n}=4255$ & $n=1389$ & $\mathrm{n}=487$ & $\mathrm{n}=246$ & $\mathrm{n}=2122$ & $\mathrm{n}=21343$ \\
\hline Sekundäre & 1476 & 469 & 178 & 60 & 18 & 256 & 2201 \\
\hline Sectio & $9,9 \%$ & $11,0 \%$ & $12,8 \%$ & $12,3 \%$ & $7,3 \%$ & $12,1 \%$ & $10,3 \%$ \\
\hline
\end{tabular}

Um schließlich den unabhängigen Einfluss eines hohen BMI von adipositas-assoziierten Erkrankungen auf das Sectio-Risiko zu überprüfen, wurden Frauen mit einer diabetischen Stoffwechsellage sowie hypertensiven Erkrankungen in der Schwangerschaft von der untersuchten Stichprobe ausgeschlossen (Tab. 31).

Insgesamt war die Kaiserschnitt-Rate in dieser Gruppe von Patientinnen (N=24516) mit 21,9\% niedriger, als im Gesamtkollektiv mit 23,5\%. Die Auswertung im Chi-Quadrat-Test zeigte, dass auch innerhalb dieser Subgruppe bei adipösen Frauen mit 30,6\% signifikant häufiger ( $<<0,001)$ ein Kaiserschnitt durchgeführt wurde als bei den Frauen des Referenzkollektivs mit 20,1\%.

Tab. 31 Absolute und relative Häufigkeiten einer Sectio caesarea in Bezug auf maternale BMI-Kategorien nach Ausschluss der Schwangeren mit adipositas-assoziierten Komorbiditäten

\begin{tabular}{lccccccc} 
& \multicolumn{7}{c}{ BMI-Kategorien $\left[\mathrm{kg} / \mathrm{m}^{2}\right]$} \\
\cline { 2 - 7 } Geburts- & $\mathbf{1 8 , 5 - 2 4 , 9}$ & $\mathbf{2 5 , 0 - 2 9 , 9}$ & $\mathbf{3 0 , 0 - 3 4 , 9}$ & $\mathbf{3 5 , 0 - 3 9 , 9}$ & $\mathbf{2 4 0}$ & $\mathbf{2 3 0}$ & Gesamt \\
modus & $\mathrm{n}=17292$ & $\mathrm{n}=4913$ & $\mathrm{n}=1554$ & $\mathrm{n}=516$ & $\mathrm{n}=241$ & $\mathrm{n}=2311$ & $\mathrm{~N}=24516$ \\
\hline \hline Sectio & 3480 & 1183 & 453 & 166 & 89 & 708 & 5371 \\
gesamt & $20,1 \%$ & $24,1 \%$ & $29,2 \%$ & $32,2 \%$ & $36,9 \%$ & $30,6 \%$ & $21,9 \%$ \\
\hline
\end{tabular}


Tabellen 32 und 33 stellen die absoluten und relativen Häufigkeiten der verschiedenen Indikationen zur primären bzw. sekundären Sectio caesarea in Bezug auf die mütterliche BMI-Kategorie dar. Dabei sind Mehrfachnennungen möglich, da Patientinnen unter Umständen aufgrund mehrerer Ursachen sectioniert wurden. Die angegebenen p-Werte beziehen sich auf den Vergleich der BMI-Gruppen Normalgewicht und Adipositas und wurden mithilfe des Chi-QuadratTests ermittelt.

Als häufigste Indikation für einen primären Kaiserschnitt in Bezug auf alle erfassten Indikationen wurde sowohl unter adipösen als auch unter normalgewichtigen Patientinnen der Z. n. Sectio mit 45,4\% bzw. 31,3\% genannt. In der Gruppe der adipösen Frauen folgten die Ursachen pathologisches/suspektes Cardiotokogramm (CTG) (16,5\%), Beckenendlage (14,8\%), mütterliche Erkrankung (11,2\%) sowie Präeklampsie und pathologischer Dopplerbefund mit jeweils 7,7\% nach Häufigkeit (Tab. 32).

Zu den Gründen, die bei adipösen Frauen signifikant häufiger Anlass zur primären Sectio gaben als bei normalgewichtigen Frauen, zählten der Z. n. Sectio (45,4\% gegenüber 31,3\%), pathologisches/suspektes CTG (16,5\% gegenüber 11,9\%), pathologischer Dopplerbefund (7,7\% gegenüber 4,1\%) und Verdacht auf (V. a.) Makrosomie (5,7\% gegenüber 3,2\%).

Mütterliche Erkrankungen insgesamt sowie speziell die Präeklampsie als Ursache für die Notwendigkeit einer abdominellen Entbindung wurden prozentual in der Gruppe der adipösen Frauen (11,2\% bzw. 7,7\%) häufiger genannt als in der Referenzgruppe (9,4\% bzw. 5,7\%). Die Unterschiede waren jedoch nicht statistisch signifikant.

Die primäre Sectio aufgrund einer Schwangerschaft in Beckenendlage wurde wiederum bei normalgewichtigen Frauen (28,3\%) signifikant häufiger durchgeführt als bei adipösen Frauen (14,8\%). Dabei sollte die unter 3.4.2. (Tab. 28) bereits beschriebene Tatsache berücksichtigt werden, dass der Anteil der Schwangerschaften mit einer kindlichen Beckenendlage insgesamt unter normalgewichtigen Frauen höher war, als unter den Patientinnen mit einem BMI $\geq 30$ $\mathrm{kg} / \mathrm{m}^{2}$.

In Bezug auf die bislang nicht erwähnten, in Tabelle 32 dargestellten Anlässe zur primären Sectio caesarea, war kein signifikanter Zusammenhang mit der mütterlichen BMI-Kategorie festzustellen. 
Tab. 32 Absolute und relative Häufigkeiten der verschiedenen Indikationen zur primären Sectio in Bezug auf maternale BMI-Kategorien

BMI-Kategorien $\left[\mathrm{kg} / \mathrm{m}^{2}\right]$

\begin{tabular}{|c|c|c|c|c|c|c|c|c|}
\hline Indikation & $\begin{array}{c}\mathbf{1 8 , 5 - 2 4 , 9} \\
n=1910\end{array}$ & $\begin{array}{c}\mathbf{2 5 , 0 - 2 9 , 9} \\
n=737\end{array}$ & $\begin{array}{c}\mathbf{3 0 , 0 - 3 4 , 9} \\
n=328\end{array}$ & $\begin{array}{c}35,0-39,9 \\
n=158\end{array}$ & $\begin{array}{l}\geq \mathbf{4 0} \\
\mathrm{n}=96\end{array}$ & $\begin{array}{c}\geq \mathbf{3 0} \\
\mathrm{n}=582\end{array}$ & $\begin{array}{r}\text { Gesamt } \\
\mathrm{N}=3229\end{array}$ & P-Wert \\
\hline Z.n. Sectio & $\begin{array}{c}597 \\
31,3 \%\end{array}$ & $\begin{array}{c}280 \\
38,0 \%\end{array}$ & $\begin{array}{c}144 \\
43,9 \%\end{array}$ & $\begin{array}{c}81 \\
51,3 \%\end{array}$ & $\begin{array}{c}39 \\
40,6 \%\end{array}$ & $\begin{array}{c}264 \\
45,4 \% \\
\end{array}$ & $\begin{array}{c}1141 \\
35,3 \% \\
\end{array}$ & $<0,001$ \\
\hline BEL & $\begin{array}{c}541 \\
28,3 \% \\
\end{array}$ & $\begin{array}{c}143 \\
19,4 \% \\
\end{array}$ & $\begin{array}{c}49 \\
14,9 \% \\
\end{array}$ & $\begin{array}{c}25 \\
15,8 \% \\
\end{array}$ & $\begin{array}{c}12 \\
12,5 \% \\
\end{array}$ & $\begin{array}{c}86 \\
14,8 \% \\
\end{array}$ & $\begin{array}{c}770 \\
23,8 \% \\
\end{array}$ & $<0,001$ \\
\hline $\begin{array}{l}\text { patholog./ } \\
\text { susp. CTG }\end{array}$ & $\begin{array}{c}227 \\
11,9 \% \\
\end{array}$ & $\begin{array}{c}89 \\
12,1 \% \\
\end{array}$ & $\begin{array}{c}52 \\
15,9 \% \\
\end{array}$ & $\begin{array}{c}30 \\
19,0 \% \\
\end{array}$ & $\begin{array}{c}14 \\
14,6 \% \\
\end{array}$ & $\begin{array}{c}96 \\
16,5 \% \\
\end{array}$ & $\begin{array}{c}412 \\
12,8 \% \\
\end{array}$ & 0,004 \\
\hline $\begin{array}{l}\text { mütterl. } \\
\text { Erkrankung }\end{array}$ & $\begin{array}{r}180 \\
9,4 \% \\
\end{array}$ & $\begin{array}{c}81 \\
11,0 \% \\
\end{array}$ & $\begin{array}{c}34 \\
10,4 \% \\
\end{array}$ & $\begin{array}{c}14 \\
8,9 \% \\
\end{array}$ & $\begin{array}{c}17 \\
17,7 \% \\
\end{array}$ & $\begin{array}{c}65 \\
11,2 \% \\
\end{array}$ & $\begin{array}{c}326 \\
10,1 \% \\
\end{array}$ & 0,216 \\
\hline $\begin{array}{l}\text { Wachstums- } \\
\text { retardierung }\end{array}$ & $\begin{array}{r}139 \\
7,3 \% \\
\end{array}$ & $\begin{array}{c}58 \\
7,9 \% \\
\end{array}$ & $\begin{array}{c}28 \\
8,5 \% \\
\end{array}$ & $\begin{array}{c}10 \\
6,3 \% \\
\end{array}$ & $\begin{array}{c}5 \\
5,2 \% \\
\end{array}$ & $\begin{array}{c}43 \\
7,4 \% \\
\end{array}$ & $\begin{array}{r}240 \\
7,4 \% \\
\end{array}$ & 0,928 \\
\hline Präeklampsie & $\begin{array}{r}108 \\
5,7 \% \\
\end{array}$ & $\begin{array}{r}46 \\
6,2 \% \\
\end{array}$ & $\begin{array}{c}21 \\
6,4 \% \\
\end{array}$ & $\begin{array}{c}14 \\
8,9 \% \\
\end{array}$ & $\begin{array}{c}10 \\
10,4 \%\end{array}$ & $\begin{array}{c}45 \\
7,7 \% \\
\end{array}$ & $\begin{array}{r}199 \\
6,2 \% \\
\end{array}$ & 0,068 \\
\hline $\begin{array}{l}\text { patholog. } \\
\text { Doppler }\end{array}$ & $\begin{array}{c}78 \\
4,1 \% \\
\end{array}$ & $\begin{array}{c}48 \\
6,5 \% \\
\end{array}$ & $\begin{array}{c}28 \\
8,5 \% \\
\end{array}$ & $\begin{array}{c}6 \\
3,8 \% \\
\end{array}$ & $\begin{array}{c}11 \\
11,5 \% \\
\end{array}$ & $\begin{array}{c}45 \\
7,7 \% \\
\end{array}$ & $\begin{array}{c}171 \\
5,3 \% \\
\end{array}$ & $<0,001$ \\
\hline $\begin{array}{l}\text { V.a. Plazenta- } \\
\text { insuffizienz }\end{array}$ & $\begin{array}{r}85 \\
4,5 \% \\
\end{array}$ & $\begin{array}{c}29 \\
3,9 \% \\
\end{array}$ & $\begin{array}{c}22 \\
6,7 \% \\
\end{array}$ & $\begin{array}{c}3 \\
1,9 \% \\
\end{array}$ & $\begin{array}{c}4 \\
4,2 \% \\
\end{array}$ & $\begin{array}{c}29 \\
5,0 \% \\
\end{array}$ & $\begin{array}{r}143 \\
4,4 \% \\
\end{array}$ & 0,590 \\
\hline $\begin{array}{l}\text { V.a. } \\
\text { Makrosomie } \\
\end{array}$ & $\begin{array}{c}62 \\
3,2 \% \\
\end{array}$ & $\begin{array}{r}30 \\
4,1 \% \\
\end{array}$ & $\begin{array}{c}15 \\
4,6 \% \\
\end{array}$ & $\begin{array}{c}12 \\
7,6 \% \\
\end{array}$ & $\begin{array}{c}6 \\
6,3 \% \\
\end{array}$ & $\begin{array}{c}33 \\
5,7 \% \\
\end{array}$ & $\begin{array}{r}125 \\
3,9 \% \\
\end{array}$ & 0,008 \\
\hline $\begin{array}{l}\text { Plazenta } \\
\text { praevia }\end{array}$ & $\begin{array}{c}59 \\
3,1 \% \\
\end{array}$ & $\begin{array}{c}16 \\
2,1 \% \\
\end{array}$ & $\begin{array}{c}11 \\
3,3 \% \\
\end{array}$ & $\begin{array}{c}3 \\
1,9 \% \\
\end{array}$ & $\begin{array}{c}2 \\
2,1 \% \\
\end{array}$ & $\begin{array}{c}16 \\
2,7 \% \\
\end{array}$ & $\begin{array}{c}91 \\
2,8 \% \\
\end{array}$ & 0,581 \\
\hline $\begin{array}{l}\text { Oligo- } \\
\text { hydramnion }\end{array}$ & $\begin{array}{c}46 \\
2,4 \% \\
\end{array}$ & $\begin{array}{c}14 \\
1,9 \% \\
\end{array}$ & $\begin{array}{c}10 \\
3,0 \% \\
\end{array}$ & $\begin{array}{c}5 \\
3,2 \% \\
\end{array}$ & $\begin{array}{c}3 \\
3,1 \% \\
\end{array}$ & $\begin{array}{c}18 \\
3,1 \% \\
\end{array}$ & $\begin{array}{c}78 \\
2,4 \% \\
\end{array}$ & 0,362 \\
\hline $\begin{array}{l}\text { Wunsch- } \\
\text { sectio }\end{array}$ & $\begin{array}{c}48 \\
2,5 \% \\
\end{array}$ & $\begin{array}{c}17 \\
2,3 \% \\
\end{array}$ & $\begin{array}{c}9 \\
2,7 \% \\
\end{array}$ & $\begin{array}{c}3 \\
1,9 \% \\
\end{array}$ & $\begin{array}{c}0 \\
0,0 \% \\
\end{array}$ & $\begin{array}{c}12 \\
2,1 \% \\
\end{array}$ & $\begin{array}{c}77 \\
2,4 \% \\
\end{array}$ & 0,534 \\
\hline $\begin{array}{l}\text { Kindl. Miss- } \\
\text { bildung }\end{array}$ & $\begin{array}{c}40 \\
2,1 \% \\
\end{array}$ & $\begin{array}{c}19 \\
2,6 \% \\
\end{array}$ & $\begin{array}{c}2 \\
0,6 \% \\
\end{array}$ & $\begin{array}{c}4 \\
2,5 \% \\
\end{array}$ & $\begin{array}{c}0 \\
0,0 \% \\
\end{array}$ & $\begin{array}{c}6 \\
1,0 \% \\
\end{array}$ & $\begin{array}{c}65 \\
2,0 \% \\
\end{array}$ & 0,095 \\
\hline $\begin{array}{l}\text { Sonstige } \\
\text { Lageanomalie }\end{array}$ & $\begin{array}{c}27 \\
1,4 \% \\
\end{array}$ & $\begin{array}{c}14 \\
1,9 \% \\
\end{array}$ & $\begin{array}{c}7 \\
2,1 \% \\
\end{array}$ & $\begin{array}{c}3 \\
1,9 \% \\
\end{array}$ & $\begin{array}{c}0 \\
0,0 \% \\
\end{array}$ & $\begin{array}{c}10 \\
1,7 \% \\
\end{array}$ & $\begin{array}{c}51 \\
1,6 \% \\
\end{array}$ & 0,952 \\
\hline Frühgeburt & $\begin{array}{c}25 \\
1,3 \% \\
\end{array}$ & $\begin{array}{c}8 \\
1,1 \%\end{array}$ & $\begin{array}{c}7 \\
2,1 \% \\
\end{array}$ & $\begin{array}{c}2 \\
1,3 \% \\
\end{array}$ & $\begin{array}{c}1 \\
1,0 \% \\
\end{array}$ & $\begin{array}{c}10 \\
1,7 \% \\
\end{array}$ & $\begin{array}{c}43 \\
1,3 \% \\
\end{array}$ & 0,466 \\
\hline $\begin{array}{l}\text { Terminüber- } \\
\text { schreitung }\end{array}$ & $\begin{array}{c}18 \\
0,9 \% \\
\end{array}$ & $\begin{array}{c}14 \\
1,9 \% \\
\end{array}$ & $\begin{array}{c}6 \\
1,8 \%\end{array}$ & $\begin{array}{c}2 \\
1,3 \%\end{array}$ & $\begin{array}{c}2 \\
2,1 \%\end{array}$ & $\begin{array}{c}10 \\
1,7 \%\end{array}$ & $\begin{array}{c}42 \\
1,3 \% \\
\end{array}$ & 0,120 \\
\hline
\end{tabular}


Unter den Indikationen zur sekundären Sectio caesarea waren die meistgenannten sowohl innerhalb der adipösen als auch unter den normalgewichtigen Gebärenden ein pathologisches bzw. suspektes CTG (46,0\% bzw. 38,0\%) sowie ein Stillstand oder protrahierter Verlauf der EP (29,7\% bzw. 24,1\%) (Tab. 33). Unter den Frauen der BMI-Kategorie Adipositas schlossen sich die Ursachen vorzeitiger Blasensprung (17,9\%), Stillstand oder protrahierter Verlauf der AP (16,7\%), Z. n. Sectio (13,9\%), sonstige Lageanomalien und Beckenendlage (9,4\% bzw. 8,8\%) sowie grünes Fruchtwasser (6,8\%) der Häufigkeitsreihenfolge nach an.

Im Vergleich zur Referenzgruppe wurden die Umstände suspektes/pathologisches CTG (46,0\% gegenüber 38,0\%), Stillstand oder protrahierter Verlauf der EP (29,7\% gegenüber 24,1\%), Z. n. Sectio (13,9\% gegenüber 10,6\%), mütterliche Erkrankung (4,2\% gegenüber 2,3\%) und V. a. Makrosomie (3,8\% gegenüber 1,5\%) bei adipösen Frauen signifikant häufiger als Anlass zur sekundären Sectio angegeben.

Ein Stillstand oder protrahierter Verlauf der AP wurde mit 19,7\% unter normalgewichtigen Müttern prozentual häufiger genannt als im Kollektiv der adipösen Frauen mit 16,7\%, wobei sich der Unterschied im Chi-Quadrat-Test als nicht statistisch signifikant erwies.

Statistisch signifikant häufiger unter normalgewichtigen Frauen im Vergleich zur adipösen Subgruppe wurden die Indikationen Beckenendlage (17,7\% gegenüber 8,8\%), sonstige Lageanomalien $(11,7 \%$ gegenüber $9,4 \%)$ und Wachstumsretardierung $(3,0 \%$ gegenüber $1,4 \%)$ angeführt. Auch hier gilt es, den bereits unter 3.4.2. (Tab. 28) beschriebenen Umstand zu berücksichtigen, dass der Anteil der Schwangerschaften mit kindlicher Beckenendlage insgesamt unter normalgewichtigen Frauen höher war, als unter adipösen Frauen.

Hinsichtlich der bislang nicht erwähnten, in Tabelle 33 aufgeführten Indikationen zur sekundären Sectio caesarea bestanden keine signifikanten Unterschiede zwischen den BMI-Gruppen. 
Tab. 33 Absolute und relative Häufigkeiten der verschiedenen Indikationen zur sekundären Sectio in Bezug auf maternale BMI-Kategorien

BMI-Kategorien $\left[\mathrm{kg} / \mathrm{m}^{2}\right]$

\begin{tabular}{|c|c|c|c|c|c|c|c|c|}
\hline \multirow{2}{*}{ Indikation } & \multicolumn{4}{|c|}{$18,5-24,925,0-29,930,0-34,935,0-39,9$} & \multirow{2}{*}{$\begin{array}{l}\geq \mathbf{4 0} \\
\mathrm{n}=72\end{array}$} & \multirow{2}{*}{$\begin{array}{c}\geq \mathbf{3 0} \\
\mathrm{n}=498\end{array}$} & \multirow{2}{*}{$\begin{array}{c}\text { Gesamt } \\
N=3303\end{array}$} & \multirow{2}{*}{ P-Wert } \\
\hline & $n=2067$ & $\mathrm{n}=738$ & $\mathrm{n}=301$ & $\mathrm{n}=125$ & & & & \\
\hline \multirow{2}{*}{$\begin{array}{l}\text { patholog./ } \\
\text { susp. CTG } \\
\end{array}$} & $\overline{7886}$ & 305 & 126 & 60 & 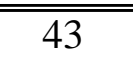 & 229 & 1320 & \multirow{2}{*}{0,001} \\
\hline & $38,0 \%$ & $41,3 \%$ & $41,9 \%$ & $48,0 \%$ & $59,7 \%$ & $46,0 \%$ & $40,0 \%$ & \\
\hline \multirow{2}{*}{$\begin{array}{l}\text { Stillstand/ } \\
\text { protrahierte EP }\end{array}$} & 499 & 188 & 89 & 33 & 26 & 148 & 835 & \multirow{2}{*}{0,010} \\
\hline & $24,1 \%$ & $25,5 \%$ & $29,6 \%$ & $26,4 \%$ & $36,1 \%$ & $29,7 \%$ & $25,3 \%$ & \\
\hline \multirow{2}{*}{$\begin{array}{l}\text { Stillstand/ } \\
\text { protrahierte AP }\end{array}$} & 408 & 147 & 52 & 21 & 10 & 83 & 638 & \multirow{2}{*}{0,118} \\
\hline & $19,7 \%$ & $19,9 \%$ & $17,3 \%$ & $16,8 \%$ & $13,9 \%$ & $16,7 \%$ & $19,3 \%$ & \\
\hline \multirow{2}{*}{$\begin{array}{l}\text { Vorzeitiger } \\
\text { Blasensprung } \\
\end{array}$} & 371 & 130 & 59 & 17 & 13 & 89 & 590 & \multirow{2}{*}{0,968} \\
\hline & $17,9 \%$ & $17,6 \%$ & $19,6 \%$ & $13,6 \%$ & $18,1 \%$ & $17,9 \%$ & $17,9 \%$ & \\
\hline \multirow{2}{*}{ BEL } & 365 & 83 & 30 & 9 & 5 & 44 & 492 & \multirow{2}{*}{$<0,001$} \\
\hline & $17,7 \%$ & $11,2 \%$ & $10,0 \%$ & $7,2 \%$ & $6,9 \%$ & $8,8 \%$ & $14,9 \%$ & \\
\hline \multirow{2}{*}{ Z.n. Sectio } & 220 & 106 & 40 & 16 & 13 & 69 & 395 & \multirow{2}{*}{0,042} \\
\hline & $10,6 \%$ & $14,4 \%$ & $13,3 \%$ & $12,8 \%$ & $18,1 \%$ & $13,9 \%$ & $12,0 \%$ & \\
\hline \multirow{2}{*}{$\begin{array}{l}\text { Sonstige } \\
\text { Lageanomalie }\end{array}$} & 241 & 79 & 32 & 10 & 5 & 47 & 367 & \multirow{2}{*}{0,034} \\
\hline & $11,7 \%$ & $10,7 \%$ & $10,6 \%$ & $8,0 \%$ & $6,9 \%$ & $9,4 \%$ & $11,1 \%$ & \\
\hline \multirow{2}{*}{$\begin{array}{l}\text { Fieber } \\
\text { sub partu }\end{array}$} & 140 & 44 & 24 & 7 & 2 & 33 & 217 & \multirow{2}{*}{0,907} \\
\hline & $6,8 \%$ & $6,0 \%$ & $8,0 \%$ & $5,6 \%$ & $2,8 \%$ & $6,6 \%$ & $6,6 \%$ & \\
\hline \multirow{2}{*}{$\begin{array}{l}\text { Grünes } \\
\text { Fruchtwasser }\end{array}$} & 110 & 43 & 16 & 11 & 7 & 34 & 187 & \multirow{2}{*}{0,190} \\
\hline & $5,3 \%$ & $5,8 \%$ & $5,3 \%$ & $8,8 \%$ & $9,7 \%$ & $6,8 \%$ & $5,7 \%$ & \\
\hline \multirow{2}{*}{$\begin{array}{l}\text { V.a. Amnionin- } \\
\text { fektionssyndrom }\end{array}$} & 99 & 36 & 18 & 4 & 2 & 24 & 159 & 023 \\
\hline & $4,8 \%$ & $4,9 \%$ & $6,0 \%$ & $3,2 \%$ & $2,8 \%$ & $4,8 \%$ & $4,8 \%$ & $0,9<3$ \\
\hline urt & 65 & 23 & 8 & 3 & 1 & 12 & 100 & 0282 \\
\hline Frun & $3,1 \%$ & $3,1 \%$ & $2,7 \%$ & $2,4 \%$ & $1,4 \%$ & $2,4 \%$ & $3,0 \%$ & 0,582 \\
\hline Wachstums- & 62 & 23 & 5 & 1 & 1 & 7 & 92 & \\
\hline retardierung & $3,0 \%$ & $3,1 \%$ & $1,7 \%$ & $0,8 \%$ & $1,4 \%$ & $1,4 \%$ & $2,8 \%$ & 0,048 \\
\hline Nabelschnur- & 51 & 27 & 7 & 2 & 2 & 11 & 89 & \\
\hline komplikationen & $2,5 \%$ & $3,7 \%$ & $2,3 \%$ & $1,6 \%$ & $2,8 \%$ & $2,2 \%$ & $2,7 \%$ & $0, / 36$ \\
\hline Mütterl. & 48 & 18 & 11 & 7 & 3 & 21 & 87 & \\
\hline Erkrankung & $2,3 \%$ & $2,4 \%$ & $3,7 \%$ & $5,6 \%$ & $4,2 \%$ & $4,2 \%$ & $2,6 \%$ & 0,019 \\
\hline Absol./rel. & 59 & 16 & 6 & 2 & 1 & 9 & 84 & \\
\hline Missvervältnis & $2,9 \%$ & $2,2 \%$ & $2,0 \%$ & $1,6 \%$ & $1,4 \%$ & $1,8 \%$ & $2,5 \%$ & 0,192 \\
\hline Terminüber- & 51 & 16 & 8 & 3 & 2 & 13 & 80 & \\
\hline schreitung & $2,5 \%$ & $2,2 \%$ & $2,7 \%$ & $2,4 \%$ & $2,8 \%$ & $2,6 \%$ & $2,4 \%$ & 0,854 \\
\hline Vorzeitige & 43 & 12 & 8 & 0 & 2 & 10 & 65 & \\
\hline Plazentalösung & $2,1 \%$ & $1,6 \%$ & $2,7 \%$ & $0,0 \%$ & $2,8 \%$ & $2,0 \%$ & $2,0 \%$ & 0,891 \\
\hline V.a. & 30 & 14 & 13 & 4 & 2 & 19 & 63 & 0001 \\
\hline Makrosomie & $1,5 \%$ & $1,9 \%$ & $4,3 \%$ & $3,2 \%$ & $2,8 \%$ & $3,8 \%$ & $1,9 \%$ & 0,001 \\
\hline & 27 & 8 & 0 & 3 & 3 & 6 & 41 & 0857 \\
\hline Izidose & $1,3 \%$ & $1,1 \%$ & $0,0 \%$ & $2,4 \%$ & $4,2 \%$ & $1,2 \%$ & $1,2 \%$ & 0,8 \\
\hline
\end{tabular}


Eine Not-Sectio wurde im Analysezeitraum insgesamt in 338 Fällen $(1,2 \%)$ durchgeführt. Dabei mussten übergewichtige (73 Fälle) und adipöse Schwangere (48 Fälle) mit 1,3\% bzw. 1,5\%, im Vergleich zur BMI-Kategorie Normalgewicht (217 Fälle) mit 1,2\%, etwas häufiger notfallmäßig sectioniert werden (Tab. 34). Statistische Signifikanz wurde im Chi-Quadrat-Test nicht bestätigt ( $\mathrm{p}=0,469$ bzw. $\mathrm{p}=0,098)$.

Tab. 34 Absolute und relative Häufigkeiten einer Not-Sectio in Bezug auf maternale BMI-Kategorien

BMI-Kategorien $\left[\mathrm{kg} / \mathrm{m}^{2}\right]$

\begin{tabular}{|c|c|c|c|c|c|c|c|}
\hline $\begin{array}{l}\text { Not- } \\
\text { sectio }\end{array}$ & $\begin{array}{r}\mathbf{1 8 , 5}-\mathbf{2 4 , 9} \\
\mathrm{n}=18809\end{array}$ & $\begin{array}{c}\mathbf{2 5 , 0 - 2 9 , 9} \\
n=5741\end{array}$ & $\begin{array}{c}\mathbf{3 0 , 0 - 3 4 , 9} \\
\mathrm{n}=2009\end{array}$ & $\begin{array}{c}\mathbf{3 5 , 0 - 3 9 , 9} \\
\mathrm{n}=774\end{array}$ & $\begin{array}{c}\geq \mathbf{4 0} \\
n=421\end{array}$ & $\begin{array}{c}\geq \mathbf{3 0} \\
n=3204\end{array}$ & $\begin{array}{c}\text { Gesamt } \\
\mathrm{N}=27754\end{array}$ \\
\hline \multirow{2}{*}{$\mathrm{Ja}$} & 217 & $\overline{773}$ & 31 & $\overline{99}$ & 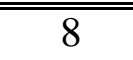 & 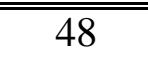 & ב338 \\
\hline & $1,2 \%$ & $1,3 \%$ & $1,5 \%$ & $1,2 \%$ & $1,9 \%$ & $1,5 \%$ & $1,2 \%$ \\
\hline Nein & $\begin{array}{l}18592 \\
98,8 \%\end{array}$ & $\begin{array}{c}5668 \\
98,7 \%\end{array}$ & $\begin{array}{c}1978 \\
98,5 \%\end{array}$ & $\begin{array}{c}765 \\
98,8 \%\end{array}$ & $\begin{array}{c}413 \\
98,1 \%\end{array}$ & $\begin{array}{c}3156 \\
98,5 \%\end{array}$ & $\begin{array}{l}27416 \\
98,8 \%\end{array}$ \\
\hline
\end{tabular}

\subsubsection{Gestationsalter bei Geburt}

Innerhalb des Gesamtkollektivs wurde die Mehrzahl der Kinder (46,5\%) mit einem Gestationsalter zwischen 37+0 und 39+6 SSW zur Welt gebracht, gefolgt von einem Schwangerschaftsalter zwischen 40+1 und 41+6 SSW, in dem 35,1\% der Entbindungen stattfanden. Der Anteil der Frühgeburten $(<37+0$ SSW) betrug insgesamt $12,2 \%$, wobei 9,9\% auf den Zeitraum zwischen $32+0$ und 36+6 SSW fielen, 1,7\% auf den Zeitraum zwischen 27+0 und 31+6 SSW und 0,6\% auf eine Tragzeit $<27+0$ SSW. 4,0\% der Kinder wurden am berechneten Entbindungstermin geboren $(40+0 \mathrm{SSW})$ und zu einer zeitlichen Übertragung der Schwangerschaft $(\geq 42+0$ SSW) kam es in insgesamt 2,3\% der Fälle.

Tabelle 35 und Abbildung 13 stellen die absoluten und relativen Häufigkeiten des in Zeiträume klassifizierten Gestationsalters in Bezug auf die mütterlichen BMI-Kategorien dar.

Ein maternaler BMI $\geq 30 \mathrm{~kg} / \mathrm{m}^{2}$ konnte positiv mit der Frühgeburtlichkeit in Verbindung gebracht werden. Innerhalb der Gruppe der adipösen Frauen wurden mit einem Anteil von 15,1\% signifikant mehr Kinder vor Vollendung der 37. Schwangerschaftswoche zur Welt gebracht als unter den normalgewichtigen Frauen mit 11,7\% ( $p<0,001)$. Auch ein Gestationsalter kleiner als 32+0 SSW war, im Vergleich zur Referenzgruppe mit 2,2\%, unter adipösen Müttern mit 2,9\% signifikant häufiger $z u$ finden $(p=0,006)$. 
Die Frühgeburt mit einem Schwangerschaftsalter kleiner als 27+0 SSW trat bei adipösen Studienteilnehmerinnen insgesamt $(0,7 \%)$ und insbesondere innerhalb der Kategorie Adipositas Grad III (1,2\%) prozentual häufiger auf als im Vergleichskollektiv (0,6\%). Im Chi-Quadrat Test bzw. exakten Test nach Fisher zeigten sich die Unterschiede jedoch als nicht statistisch signifikant ( $\mathrm{p}=0,624$ bzw. $\mathrm{p}=0,111$ ).

Die Kinder von übergewichtigen (38,3\%) und adipösen Schwangeren (37,3\%) wurden insgesamt etwas häufiger nach dem errechneten Geburtstermin ( $\geq 40+1 \mathrm{SSW})$ geboren als die Kinder normalgewichtiger Patientinnen (37,1\%), die Unterschiede beruhten jedoch nicht auf einer statistischen Signifikanz ( $\mathrm{p}=0,110$ bzw. $\mathrm{p}=0,883$ ). Hinsichtlich einer zeitlichen Übertragung der Schwangerschaft $(\geq 42+0 \mathrm{SSW})$ zeigten sich jedoch signifikante Unterschiede zwischen übergewichtigen und adipösen Frauen mit 2,6\% bzw. 2,7\% im Vergleich zur Referenzgruppe mit 2,1\% ( $\mathrm{p}=0,019$ bzw. $\mathrm{p}=0,039$; Chi-Quadrat-Test). Dabei war der Anteil der übertragenen Schwangerschaften unter den Patientinnen der Kategorie Adipositas Grad III mit 5,0\% am höchsten.

Tab. 35 Absolute und relative Häufigkeiten des in Zeiträume klassifizierten Gestationsalters in Bezug auf maternale BMI-Kategorien

BMI-Kategorien $\left[\mathrm{kg} / \mathrm{m}^{2}\right]$

\begin{tabular}{|c|c|c|c|c|c|c|c|}
\hline \multirow[b]{2}{*}{$\begin{array}{l}\text { Gestations- } \\
\text { alter [SSW] }\end{array}$} & & \multirow[b]{2}{*}{$\begin{array}{r}\text { Gesamt } \\
\mathrm{N}=27737\end{array}$} \\
\hline & $\begin{array}{c}\mathbf{1 8 , 5 - 2 4 , 9} \\
\mathrm{n}=18797\end{array}$ & $\begin{array}{c}\mathbf{2 5 , 0 - 2 9 , 9} \\
\mathrm{n}=5739\end{array}$ & $\begin{array}{c}\mathbf{3 0 , 0 - 3 4 , 9} \\
\mathrm{n}=2008\end{array}$ & $\begin{array}{c}\mathbf{3 5 , 0 - 3 9 , 9} \\
n=774\end{array}$ & $\begin{array}{c}\geq \mathbf{4 0} \\
\mathrm{n}=419\end{array}$ & $\begin{array}{c}\geq \mathbf{3 0} \\
n=3201\end{array}$ & \\
\hline \multirow{2}{*}{$\geq 42+0$} & 391 & 149 & 46 & 18 & 21 & 85 & 625 \\
\hline & $2,1 \%$ & $2,6 \%$ & $2,3 \%$ & $2,3 \%$ & $5,0 \%$ & $2,7 \%$ & $2,3 \%$ \\
\hline \multirow{2}{*}{$40+1-41+6$} & 6581 & 2046 & 690 & 268 & 149 & 1107 & 9734 \\
\hline & $35,0 \%$ & $35,7 \%$ & $34,4 \%$ & $34,6 \%$ & $35,6 \%$ & $34,6 \%$ & $35,1 \%$ \\
\hline \multirow{2}{*}{$40+0$} & 748 & 243 & 79 & 29 & 13 & 121 & 1112 \\
\hline & $4,0 \%$ & $4,2 \%$ & $3,9 \%$ & $3,7 \%$ & $3,1 \%$ & $3,8 \%$ & $4,0 \%$ \\
\hline \multirow{2}{*}{$37+0-39+6$} & 8877 & 2606 & 884 & 346 & 175 & 1405 & 12888 \\
\hline & $47,2 \%$ & $45,4 \%$ & $44,0 \%$ & $44,7 \%$ & $41,8 \%$ & $43,9 \%$ & $46,5 \%$ \\
\hline \multirow{2}{*}{$32+0-36+6$} & 1795 & 563 & 255 & 91 & 43 & 389 & 2747 \\
\hline & $9,5 \%$ & $9,8 \%$ & $12,7 \%$ & $11,8 \%$ & $10,3 \%$ & $12,2 \%$ & $9,9 \%$ \\
\hline \multirow{2}{*}{$27+0-31+6$} & 294 & 95 & 41 & 17 & 13 & 71 & 460 \\
\hline & $1,6 \%$ & $1,7 \%$ & $2,0 \%$ & $2,2 \%$ & $3,1 \%$ & $2,2 \%$ & $1,7 \%$ \\
\hline \multirow{2}{*}{$<27+0$} & 111 & 37 & 13 & 5 & 5 & 23 & 171 \\
\hline & $0,6 \%$ & $0,6 \%$ & $0,6 \%$ & $0,6 \%$ & $1,2 \%$ & $0,7 \%$ & $0,6 \%$ \\
\hline$<37+0$ & 2200 & 695 & 309 & 113 & 61 & 483 & 3378 \\
\hline gesamt & $11,7 \%$ & $12,1 \%$ & $15,3 \%$ & $14,6 \%$ & $14,6 \%$ & $15,1 \%$ & $12,2 \%$ \\
\hline
\end{tabular}




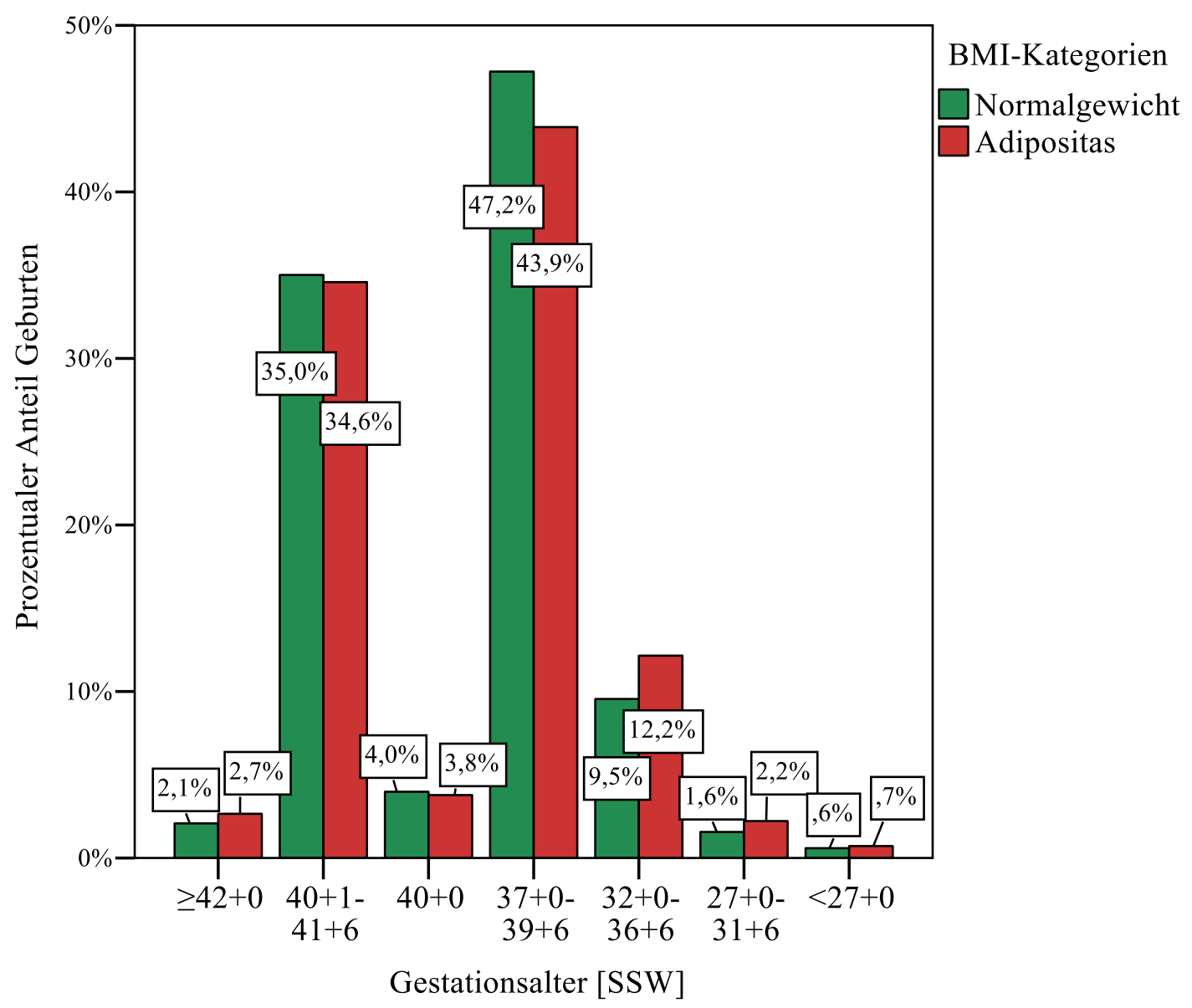

Abb. 13 Relative Häufigkeitsverteilung des in Zeiträume klassifizierten Gestationsalters in Bezug auf maternale BMI-Kategorien $(N=27737)$

Um den unabhängigen Einfluss der Adipositas von assoziierten Komorbiditäten der Schwangeren auf das Gestationsalter zu prüfen, wurde eine Analyse in der Subgruppe der Frauen nach Ausschluss der Fälle eines Diabetes mellitus und einer hypertensiven Schwangerschaftserkrankung durchgeführt $(\mathrm{N}=24470)$.

Die zugehörigen Ergebnisse der Auswertung sind in Tabelle 36 dargestellt.

Die im Gesamtkollektiv beobachtete positive Assoziation der Adipositas mit der Frühgeburtlichkeit konnte dabei als statistisch signifikant bestätigt werden, was auf einen unabhängigen Effekt der maternalen Fettleibigkeit von den genannten Komorbiditäten auf das Risiko einer Frühgeburt hindeutete. 
Im Vergleich zum Gesamtkollektiv mit 12,2\% war innerhalb der definierten Stichprobe der Anteil der Frühgeburten mit insgesamt 11,3\% niedriger (2766 Fälle), wobei 9,3\% (2265 Fälle) der frühgeborenen Kinder ein Gestationsalter zwischen 32+0 und 36+6 SSW aufwiesen, 1,4\% (349 Fälle) ein Schwangerschaftsalter zwischen 27+0 und 31+6 SSW hatten und 0,6\% der Kinder (152 Fälle) vor Vollendung der 27. SSW zur Welt kamen.

Im Vergleich zur Referenzgruppe mit 11,0\% bzw. 2,0\%, wurden die Kinder von adipösen Frauen mit einem Anteil von 14,1\% bzw. 3,0\% signifikant häufiger sowohl vor Vollendung der 37. SSW als auch vor Vollendung der 32. SSW zur Welt gebracht $(\mathrm{p}<0,001$ bzw. $p=0,001$; ChiQuadrat-Test). Die Rate der Frühgeburt mit einer Tragzeit kleiner als 27+0 SSW stieg mit der mütterlichen BMI-Kategorie auf einen Höchstwert von 2,1\% (5 Fälle) unter den Frauen mit einem BMI $\geq 40 \mathrm{~kg} / \mathrm{m}^{2}$ an. In der Gruppe der adipösen Frauen in ihrer Gesamtheit war der Anteil mit 1,0\% (22 Fälle) höher als in der normalgewichtigen Vergleichsgruppe mit 0,6\% (97 Fälle). Der Unterschied zeigte sich in dieser Subgruppenanalyse, im Gegensatz zur analogen Auswertung im Gesamtkollektiv, im Chi-Quadrat-Test als statistisch signifikant ( $\mathrm{p}=0,021)$.

Tab. 36 Absolute und relative Häufigkeiten des in Zeiträume klassifizierten Gestationsalters in Bezug auf maternale BMI-Kategorien nach Ausschluss der Schwangeren mit Diabetes mellitus oder einer hypertensiven Schwangerschaftserkrankung

BMI-Kategorien $\left[\mathrm{kg} / \mathrm{m}^{2}\right]$

\begin{tabular}{lccccccc} 
Gestations- & $\mathbf{1 8 , 5 - 2 4 , 9}$ & $\mathbf{2 5 , 0 - 2 9 , 9}$ & $\mathbf{3 0 , 0 - 3 4 , 9}$ & $\mathbf{3 5 , 0 - 3 9 , 9}$ & $\mathbf{2 4 0}$ & $\mathbf{2 3 0}$ & Gesamt \\
alter [SSW] & $\mathrm{n}=17275$ & $\mathrm{n}=4903$ & $\mathrm{n}=1543$ & $\mathrm{n}=511$ & $\mathrm{n}=238$ & $\mathrm{n}=2292$ & $\mathrm{~N}=24470$ \\
\hline \hline \multirow{2}{*}{$42+0$} & 377 & 142 & 40 & 14 & 15 & 69 & 588 \\
& $2,2 \%$ & $2,9 \%$ & $2,6 \%$ & $2,7 \%$ & $6,3 \%$ & $3,0 \%$ & $2,4 \%$ \\
\hline \multirow{2}{*}{$40+1-41+6$} & 6162 & 1802 & 547 & 185 & 92 & 824 & 8788 \\
& $35,7 \%$ & $36,8 \%$ & $35,5 \%$ & $36,2 \%$ & $38,7 \%$ & $36,0 \%$ & $35,9 \%$ \\
\hline \multirow{2}{*}{$40+0$} & 697 & 210 & 59 & 18 & 5 & 82 & 989 \\
& $4,0 \%$ & $4,3 \%$ & $3,8 \%$ & $3,5 \%$ & $2,1 \%$ & $3,6 \%$ & $4,0 \%$ \\
\hline \multirow{2}{*}{$37+0-39+6$} & 8147 & 2197 & 673 & 230 & 92 & 995 & 11339 \\
& $47,2 \%$ & $44,8 \%$ & $43,6 \%$ & $45,0 \%$ & $38,7 \%$ & $43,4 \%$ & $46,3 \%$ \\
\hline \multirow{2}{*}{$32+0-36+6$} & 1555 & 456 & 180 & 51 & 23 & 254 & 2265 \\
& $9,0 \%$ & $9,3 \%$ & $11,7 \%$ & $10,0 \%$ & $9,7 \%$ & $11,1 \%$ & $9,3 \%$ \\
\hline \multirow{2}{*}{$27+0-31+6$} & 240 & 63 & 32 & 8 & 6 & 46 & 349 \\
& $1,4 \%$ & $1,3 \%$ & $2,1 \%$ & $1,6 \%$ & $2,5 \%$ & $2,0 \%$ & $1,4 \%$ \\
\hline \multirow{2}{*}{$27+0$} & 97 & 33 & 12 & 5 & 5 & 22 & 152 \\
& $0,6 \%$ & $0,7 \%$ & $0,8 \%$ & $1,0 \%$ & $2,1 \%$ & $1,0 \%$ & $0,6 \%$ \\
\hline \hline$<37+0$ & 1892 & 552 & 224 & 64 & 34 & 322 & 2766 \\
gesamt & $11,0 \%$ & $11,3 \%$ & $14,6 \%$ & $12,6 \%$ & $14,3 \%$ & $14,1 \%$ & $11,3 \%$ \\
\hline
\end{tabular}




\subsubsection{Geburtseinleitung und Indikationen}

Insgesamt wurde im Untersuchungszeitraum bei 6404 Frauen die Geburt eingeleitet, was einem Anteil von $23,1 \%$ an allen untersuchten Geburten entsprach.

Die Häufigkeit einer Geburtseinleitung nahm mit steigendem BMI der Schwangeren zu und wurde bei übergewichtigen mit 25,8\% und bei adipösen Patientinnen mit 33,7\% signifikant häufiger durchgeführt als bei den Frauen des Referenzkollektivs mit 20,4\% (jeweils p<0,001; Chi-Quadrat-Test). Innerhalb der Gruppe der adipösen Frauen stieg der Anteil der eingeleiteten Geburten von 30,9\% unter Frauen mit Adipositas Grad I auf 37,1\% bzw. 41,3\% unter Frauen der BMI-Gruppe Adipositas Grad II und III an (Tab. 37; Abb. 14).

Tab. 37 Absolute und relative Häufigkeiten einer Geburtseinleitung in Bezug auf maternale BMI-Kategorien

\begin{tabular}{lccccccc} 
& \multicolumn{7}{c}{ BMI-Kategorien $\left[\mathrm{kg} / \mathrm{m}^{2}\right]$} \\
Geburts- & $\mathbf{1 8 , 5 - 2 4 , 9}$ & $\mathbf{2 5 , 0 - 2 9 , 9}$ & $\mathbf{3 0 , 0 - 3 4 , 9}$ & $\mathbf{3 5 , 0 - 3 9 , 9}$ & $\mathbf{2 4 0}$ & $\mathbf{2 3 0}$ & \multirow{2}{*}{ Gesamt } \\
einleitung & $\mathrm{n}=18809$ & $\mathrm{n}=5741$ & $\mathrm{n}=2009$ & $\mathrm{n}=774$ & $\mathrm{n}=421$ & $\mathrm{n}=3204$ & $\mathrm{~N}=27754$ \\
\hline \hline \multirow{2}{*}{ Ja } & 3840 & 1483 & 620 & 287 & 174 & 1081 & 6404 \\
& $20,4 \%$ & $25,8 \%$ & $30,9 \%$ & $37,1 \%$ & $41,3 \%$ & $33,7 \%$ & $23,1 \%$ \\
\hline \multirow{2}{*}{ Nein } & 14969 & 4258 & 1389 & 487 & 247 & 2123 & 21350 \\
& $79,6 \%$ & $74,2 \%$ & $69,1 \%$ & $62,9 \%$ & $58,7 \%$ & $66,3 \%$ & $76,9 \%$ \\
\hline
\end{tabular}

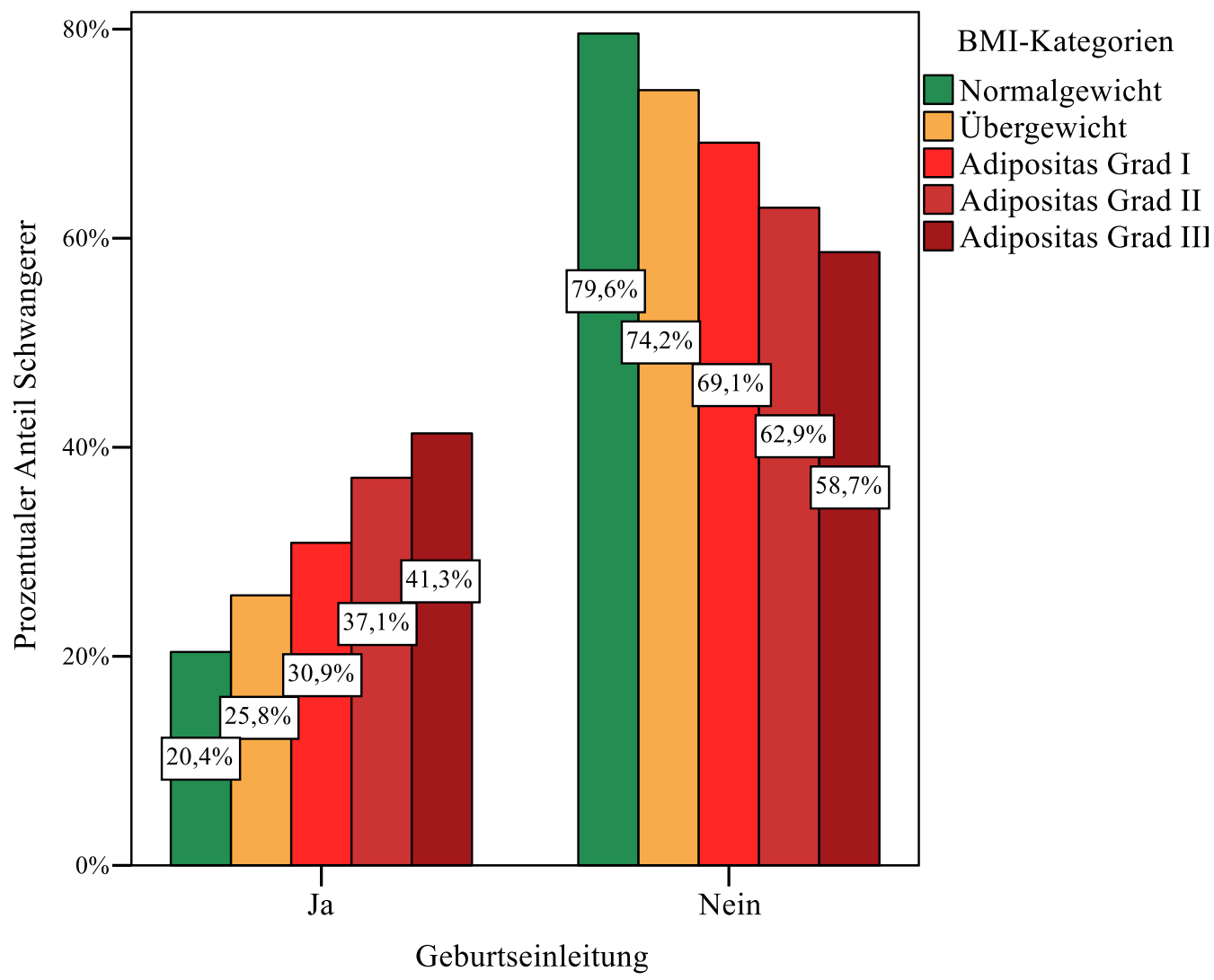

Abb. 14 Relative Häufigkeitsverteilung der Geburtseinleitung in Bezug auf maternale BMI-Kategorien (N=27754) 
Diese Beobachtung blieb auch in den Subgruppenanalysen bzgl. der Parität der Frau sowie nach Ausschluss der Frauen mit einer diabetischen Stoffwechsellage oder hypertensiven Erkrankung in der Schwangerschaft signifikant bestehen. In dieser Studie konnte demnach von einem unabhängigen Einfluss eines hohen BMI der Schwangeren von den genannten Faktoren auf die Häufigkeit einer Geburtseinleitung ausgegangen werden (Tab. 38 bis 39).

Bei der Darstellung der Frequenz einer Geburtseinleitung, stratifiziert nach der Parität der Frau, war zu beobachten, dass der Anteil der Frauen, deren Geburt eingeleitet wurde, unter Primiparae mit 26,0\% signifikant höher war als unter Pluriparae mit 20,1\%. Sowohl in der Gruppe der Primi- als auch der Pluriparae war die Rate der Geburtseinleitungen unter übergewichtigen (31,0\% bzw. 21,7\%) und adipösen Frauen (39,5\% bzw. 29,6\%) signifikant höher als unter den Frauen des Referenzkollektivs mit 22,9\% bzw. 17,5\%. Der jeweils durchgeführte Chi-QuadratTest bestätigte mit $\mathrm{p}<0,001$ in allen Fällen statistische Signifikanz. (Tab. 38).

Nach Ausschluss der Frauen mit einer diabetischen Stoffwechsellage oder hypertensiven Erkrankung in der Schwangerschaft, sank die Rate der Geburtseinleitungen insgesamt von 23,1\% im Gesamtkollektiv auf 21,1\% ab. In dieser Subgruppe von Patientinnen (N=24487) war der Anteil der Geburtseinleitungen unter übergewichtigen mit 23,7\% und unter adipösen Schwangeren mit 29,2\% weiterhin signifikant höher (jeweils $\mathrm{p}<0,001$; Chi-Quadrat-Test) als innerhalb der normalgewichtigen Vergleichsgruppe mit 19,3\% (Tab. 39).

Tab. 38 Absolute und relative Häufigkeiten einer Geburtseinleitung in Bezug auf maternale BMI-Kategorien, stratifiziert nach Parität

BMI-Kategorien $\left[\mathrm{kg} / \mathrm{m}^{2}\right]$

\begin{tabular}{cccccccc} 
Geburts- & $\mathbf{1 8 , 5 - 2 4 , 9}$ & $\mathbf{2 5 , 0 - 2 9 , 9}$ & $\mathbf{3 0 , 0 - 3 4 , 9}$ & $\mathbf{3 5 , 0 - 3 9 , 9}$ & $\mathbf{2 4 0}$ & $\mathbf{2 3 0}$ & Gesamt \\
einleitung & $\mathrm{n}=18809$ & $\mathrm{n}=5741$ & $\mathrm{n}=2009$ & $\mathrm{n}=774$ & $\mathrm{n}=421$ & $\mathrm{n}=3204$ & $\mathrm{~N}=27754$ \\
\hline Primipara & $\mathrm{n}=10007$ & $\mathrm{n}=2567$ & $\mathrm{n}=846$ & $\mathrm{n}=311$ & $\mathrm{n}=175$ & $\mathrm{n}=1332$ & $\mathrm{n}=13906$ \\
\hline \hline \multirow{2}{*}{ Ja } & 2296 & 795 & 299 & 142 & 85 & 526 & 3617 \\
& $22,9 \%$ & $31,0 \%$ & $35,3 \%$ & $45,7 \%$ & $48,6 \%$ & $39,5 \%$ & $26,0 \%$ \\
\hline \multirow{2}{*}{ Nein } & 7711 & 1772 & 547 & 169 & 90 & 806 & 10289 \\
& $77,1 \%$ & $69,0 \%$ & $64,7 \%$ & $54,3 \%$ & $51,4 \%$ & $60,5 \%$ & $74,0 \%$ \\
\hline \hline \multirow{2}{*}{ Pluripara } & $\mathrm{n}=8802$ & $\mathrm{n}=3174$ & $\mathrm{n}=1163$ & $\mathrm{n}=463$ & $\mathrm{n}=246$ & $\mathrm{n}=1872$ & $\mathrm{n}=13848$ \\
\hline \hline \multirow{2}{*}{ Ja } & 1544 & 688 & 321 & 145 & 89 & 555 & 2787 \\
& $17,5 \%$ & $21,7 \%$ & $27,6 \%$ & $31,3 \%$ & $36,2 \%$ & $29,6 \%$ & $20,1 \%$ \\
\hline \multirow{2}{*}{ Nein } & 7258 & 2486 & 842 & 318 & 157 & 1317 & 11061 \\
& $82,5 \%$ & $78,3 \%$ & $72,4 \%$ & $68,7 \%$ & $63,8 \%$ & $70,4 \%$ & $79,9 \%$ \\
\hline
\end{tabular}


Tab. 39 Absolute und relative Häufigkeiten einer Geburtseinleitung in Bezug auf maternale BMI-Kategorien nach Ausschluss der Frauen mit Diabetes mellitus oder hypertensiven Erkrankungen in der Schwangerschaft

BMI-Kategorien $\left[\mathrm{kg} / \mathrm{m}^{2}\right]$

\begin{tabular}{lccccccc} 
Geburts- & $\mathbf{1 8 , 5 - 2 4 , 9}$ & $\mathbf{2 5 , 0 - 2 9 , 9}$ & $\mathbf{3 0 , 0 - 3 4 , 9}$ & $\mathbf{3 5 , 0 - 3 9 , 9}$ & $\mathbf{2 4 0}$ & $\mathbf{2} \mathbf{3 0}$ & Gesamt \\
einleitung & $\mathrm{n}=17287$ & $\mathrm{n}=4905$ & $\mathrm{n}=1544$ & $\mathrm{n}=511$ & $\mathrm{n}=240$ & $\mathrm{n}=2295$ & $\mathrm{~N}=24487$ \\
\hline \hline \multirow{2}{*}{ Ja } & 3334 & 1163 & 423 & 163 & 83 & 669 & 5166 \\
& $19,3 \%$ & $23,7 \%$ & $27,4 \%$ & $31,9 \%$ & $34,6 \%$ & $29,2 \%$ & $21,1 \%$ \\
\hline \multirow{2}{*}{ Nein } & 13953 & 3742 & 1121 & 348 & 157 & 1626 & 19321 \\
& $80,7 \%$ & $76,3 \%$ & $72,6 \%$ & $68,1 \%$ & $65,4 \%$ & $70,8 \%$ & $78,9 \%$ \\
\hline
\end{tabular}

Die absoluten und relativen Häufigkeiten der verschiedenen Gründe, die Anlass zu einer Geburtseinleitung gaben, sind in Tabelle 40 aufgelistet. Dabei sind Mehrfachnennungen möglich, da eine Geburt möglicherweise aufgrund mehrerer Indikationen eingeleitet wurde. Die angegebenen p-Werte beziehen sich auf den Vergleich der BMI-Gruppen Normalgewicht und Adipositas und wurden mithilfe des Chi-Quadrat-Tests sowie des exakten Tests nach Fisher ermittelt.

Die häufigsten Indikationen zur Geburtseinleitung in Bezug auf alle erfassten Indikationen waren sowohl in der Kategorie Normalgewicht als auch in der Kategorie Adipositas der vorzeitige Blasensprung (37,9\% bzw. 29,0\%) und die Terminüberschreitung (26,4\% bzw. 26,5\%). Unter den Patientinnen mit einem BMI $\geq 30 \mathrm{~kg} / \mathrm{m}^{2}$ folgten die Ursachen mütterlicher Diabetes mellitus (10,6\%), Oligohydramnion (8,6\%), V. a. Makrosomie (7,8\%), mütterliche Erkrankung (7,2\%), Präeklampsie $(7,1 \%)$ und fetale Wachstumsretardierung $(3,9 \%)$ nach Häufigkeit.

$\mathrm{Zu}$ den Gründen, die unter adipösen Frauen signifikant häufiger angegeben wurden als unter denen des Referenzkollektivs, zählten mütterlicher Diabetes mellitus (10,6\% gegenüber 2,0\%), V. a. Makrosomie (7,8\% gegenüber 2,9\%), mütterliche Erkrankung (7,2\% gegenüber 3,7\%) und Präeklampsie (7,1\% gegenüber 3,8\%).

$\mathrm{Zu}$ den Indikationen, die unter fettleibigen Frauen dagegen signifikant seltener angeführt wurden als unter normalgewichtigen Frauen, zählten vorzeitiger Blasensprung (29,0\% gegenüber $37,9 \%)$, Wachstumsretardierung (3,9\% gegenüber 6,6\%), pathologisches/suspektes CTG $(2,4 \%$ gegenüber $4,9 \%)$ und V. a. Plazentainsuffizienz (2,2\% gegenüber $3,8 \%)$.

Bzgl. der bisher nicht erwähnten, in Tabelle 40 aufgeführten Indikationen zur Geburtseinleitung bestanden keine signifikanten Unterschiede zwischen den BMI-Gruppen. 
Tab. 40 Absolute und relative Häufigkeiten der verschiedenen Indikationen zur Geburtseinleitung in Bezug auf maternale BMI-Kategorien

BMI-Kategorien $\left[\mathrm{kg} / \mathrm{m}^{2}\right]$

\begin{tabular}{|c|c|c|c|c|c|c|c|c|}
\hline Indikation & $\begin{array}{c}\mathbf{1 8 , 5 - 2 4 , 9} \\
n=3840\end{array}$ & $\begin{array}{c}\mathbf{2 5 , 0 - 2 9 , 9} \\
n=1483\end{array}$ & $\begin{array}{c}\mathbf{3 0 , 0 - 3 4 , 9} \\
\mathrm{n}=620\end{array}$ & $\begin{array}{c}\mathbf{3 5 , 0 - 3 9 , 9} \\
n=287\end{array}$ & $\begin{array}{c}\geq 40 \\
\mathrm{n}=174\end{array}$ & $\begin{array}{c}\geq \mathbf{3 0} \\
\mathrm{n}=1081\end{array}$ & $\begin{array}{c}\text { Gesamt } \\
\mathrm{N}=6404\end{array}$ & P-Wert \\
\hline$\overline{\text { Vorzeitiger }}$ & 1456 & 500 & 199 & $\overline{72}$ & 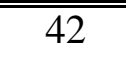 & 313 & 2269 & \\
\hline Blasensprung & $37,9 \%$ & $33,7 \%$ & $32,1 \%$ & $25,1 \%$ & $24,1 \%$ & $29,0 \%$ & $35,4 \%$ & $<0,001$ \\
\hline $\begin{array}{l}\text { Terminüber- } \\
\text { schreitung }\end{array}$ & $\begin{array}{c}1013 \\
26,4 \%\end{array}$ & $\begin{array}{c}390 \\
26,3 \%\end{array}$ & $\begin{array}{c}157 \\
25,3 \%\end{array}$ & $\begin{array}{c}77 \\
26,8 \%\end{array}$ & $\begin{array}{c}52 \\
29,9 \%\end{array}$ & $\begin{array}{c}286 \\
26,5 \%\end{array}$ & $\begin{array}{c}1689 \\
26,4 \%\end{array}$ & 0,960 \\
\hline $\begin{array}{l}\text { Oligo- } \\
\text { hydramnion }\end{array}$ & $\begin{array}{c}388 \\
10,1 \%\end{array}$ & $\begin{array}{c}144 \\
9,7 \%\end{array}$ & $\begin{array}{c}60 \\
9,7 \%\end{array}$ & $\begin{array}{c}22 \\
7,7 \%\end{array}$ & $\begin{array}{c}11 \\
6,3 \%\end{array}$ & $\begin{array}{c}93 \\
8,6 \%\end{array}$ & $\begin{array}{c}625 \\
9,8 \%\end{array}$ & 0,148 \\
\hline $\begin{array}{l}\text { Wachstums- } \\
\text { retardierung }\end{array}$ & $\begin{array}{c}253 \\
6,6 \%\end{array}$ & $\begin{array}{c}78 \\
5,3 \%\end{array}$ & $\begin{array}{c}23 \\
3,7 \%\end{array}$ & $\begin{array}{c}14 \\
4,9 \%\end{array}$ & $\begin{array}{c}5 \\
2,9 \%\end{array}$ & $\begin{array}{c}42 \\
3,9 \%\end{array}$ & $\begin{array}{c}373 \\
5,8 \%\end{array}$ & 0,001 \\
\hline Mütterl. & 143 & 73 & 40 & 23 & 15 & 78 & 294 & \\
\hline Erkrankung & $3,7 \%$ & $4,9 \%$ & $6,5 \%$ & $8,0 \%$ & $8,6 \%$ & $7,2 \%$ & $4,6 \%$ & $<0,001$ \\
\hline Präeklampsie & $\begin{array}{r}144 \\
3,8 \% \\
\end{array}$ & $\begin{array}{c}70 \\
4,7 \% \\
\end{array}$ & $\begin{array}{c}40 \\
6,5 \% \\
\end{array}$ & $\begin{array}{c}23 \\
8,0 \% \\
\end{array}$ & $\begin{array}{c}14 \\
8,0 \%\end{array}$ & $\begin{array}{c}77 \\
7,1 \% \\
\end{array}$ & $\begin{array}{c}291 \\
4,5 \% \\
\end{array}$ & $<0,001$ \\
\hline V.a. & 112 & 87 & 42 & 26 & 16 & 84 & 283 & \\
\hline Makrosomie & $2,9 \%$ & $5,9 \%$ & $6,8 \%$ & $9,1 \%$ & $9,2 \%$ & $7,8 \%$ & $4,4 \%$ & $<0,001$ \\
\hline Mütterl. & 78 & 72 & 53 & 29 & 33 & 115 & 265 & \\
\hline Diabetes & $2,0 \%$ & $4,9 \%$ & $8,5 \%$ & $10,1 \%$ & $19,0 \%$ & $10,6 \%$ & $4,1 \%$ & $<0,001$ \\
\hline patholog./ & 189 & 49 & 15 & 5 & 6 & 26 & 264 & \\
\hline susp. CTG & $4,9 \%$ & $3,3 \%$ & $2,4 \%$ & $1,7 \%$ & $3,4 \%$ & $2,4 \%$ & $4,1 \%$ & $<0,001$ \\
\hline $\begin{array}{l}\text { V.a. Plazenta- } \\
\text { insuffizienz }\end{array}$ & $\begin{array}{r}147 \\
3,8 \% \\
\end{array}$ & $\begin{array}{c}42 \\
2,8 \% \\
\end{array}$ & $\begin{array}{c}13 \\
2,1 \% \\
\end{array}$ & $\begin{array}{c}7 \\
2,4 \% \\
\end{array}$ & $\begin{array}{c}4 \\
2,3 \% \\
\end{array}$ & $\begin{array}{c}24 \\
2,2 \% \\
\end{array}$ & $\begin{array}{r}213 \\
3,3 \% \\
\end{array}$ & 0,011 \\
\hline Intrauteriner & 52 & 10 & 11 & 3 & 2 & 16 & 78 & \\
\hline Fruchttod & $1,4 \%$ & $0,7 \%$ & $1,8 \%$ & $1,0 \%$ & $1,1 \%$ & $1,5 \%$ & $1,2 \%$ & 0,754 \\
\hline Poly- & 20 & 16 & 6 & 1 & 1 & 8 & 44 & \\
\hline hydramnion & $0,5 \%$ & $1,1 \%$ & $1,0 \%$ & $0,3 \%$ & $0,6 \%$ & $0,7 \%$ & $0,7 \%$ & 0,420 \\
\hline patholog. & 24 & 10 & 2 & 1 & 2 & 5 & 39 & \\
\hline Dopplerbefund & $0,6 \%$ & $0,7 \%$ & $0,3 \%$ & $0,3 \%$ & $1,1 \%$ & $0,5 \%$ & $0,6 \%$ & 0,538 \\
\hline
\end{tabular}

\subsubsection{Wehenmittel}

Bei der Geburt von insgesamt 41,6\% der Patientinnen kam ein Wehenmittel in Form eines Oxytocin-Tropfs zum Einsatz, wobei bei dieser Analyse die Entbindungen per primärer Sectio caesarea von der untersuchten Stichprobe ausgeschlossen waren $(\mathrm{N}=24518)$.

Eine signifikante Assoziation zwischen dem mütterlichen BMI und der Inanspruchnahme eines Wehenmittels konnte dabei im Chi-Quadrat-Test festgestellt werden. Der Anteil der Frauen, bei denen während der Geburt ein Oxytocin-Tropf eingesetzt wurde, war, im Vergleich zur Referenzgruppe mit 40,8\%, unter übergewichtigen mit 42,2\% und adipösen Gebärenden in ihrer Gesamtheit mit 45,2\% höher ( $\mathrm{p}=0,092$ bzw. $\mathrm{p}<0,001)$. 
Zudem stieg der Prozentsatz mit Zunahme des BMI innerhalb der adipösen Frauen an und erreichte unter den Patientinnen mit Adipositas Grad III einen Wert von 48,5\%.

Die absoluten und relativen Häufigkeiten des Einsatzes eines Wehenmittels unter der Geburt sind in Tabelle 41 und Abbildung 15 dargestellt.

Tab. 41 Absolute und relative Häufigkeiten des Einsatzes eines Wehenmittels unter der Geburt in Bezug auf maternale BMI-Kategorien

BMI-Kategorien $\left[\mathrm{kg} / \mathrm{m}^{2}\right]$

\begin{tabular}{|c|c|c|c|c|c|c|c|}
\hline Wehenmittel & $\begin{array}{c}\mathbf{1 8 , 5 - 2 4 , 9} \\
\mathrm{n}=16896\end{array}$ & $\begin{array}{c}\mathbf{2 5 , 0 - 2 9 , 9} \\
\mathrm{n}=5001\end{array}$ & $\begin{array}{c}\mathbf{3 0 , 0 - 3 4 , 9} \\
n=1681\end{array}$ & $\begin{array}{c}\mathbf{3 5 , 0 - 3 9 , 9} \\
\mathrm{n}=616\end{array}$ & $\begin{array}{c}\geq 40 \\
n=324\end{array}$ & $\begin{array}{c}\geq \mathbf{3 0} \\
n=2621\end{array}$ & $\begin{array}{c}\text { Gesamt } \\
\mathrm{N}=24518\end{array}$ \\
\hline \multirow{2}{*}{$\mathrm{Ja}$} & "6900 & 2109 & $\overline{7746}$ & 281 & 157 & 1184 & 10193 \\
\hline & $40,8 \%$ & $42,2 \%$ & $44,4 \%$ & $45,6 \%$ & $48,5 \%$ & $45,2 \%$ & $41,6 \%$ \\
\hline Nein & $\begin{array}{c}9996 \\
59,2 \%\end{array}$ & $\begin{array}{c}2892 \\
57,8 \%\end{array}$ & $\begin{array}{c}935 \\
55,6 \%\end{array}$ & $\begin{array}{c}335 \\
54,4 \%\end{array}$ & $\begin{array}{c}167 \\
51,5 \%\end{array}$ & $\begin{array}{c}1437 \\
54,8 \%\end{array}$ & $\begin{array}{l}14325 \\
58,4 \%\end{array}$ \\
\hline
\end{tabular}

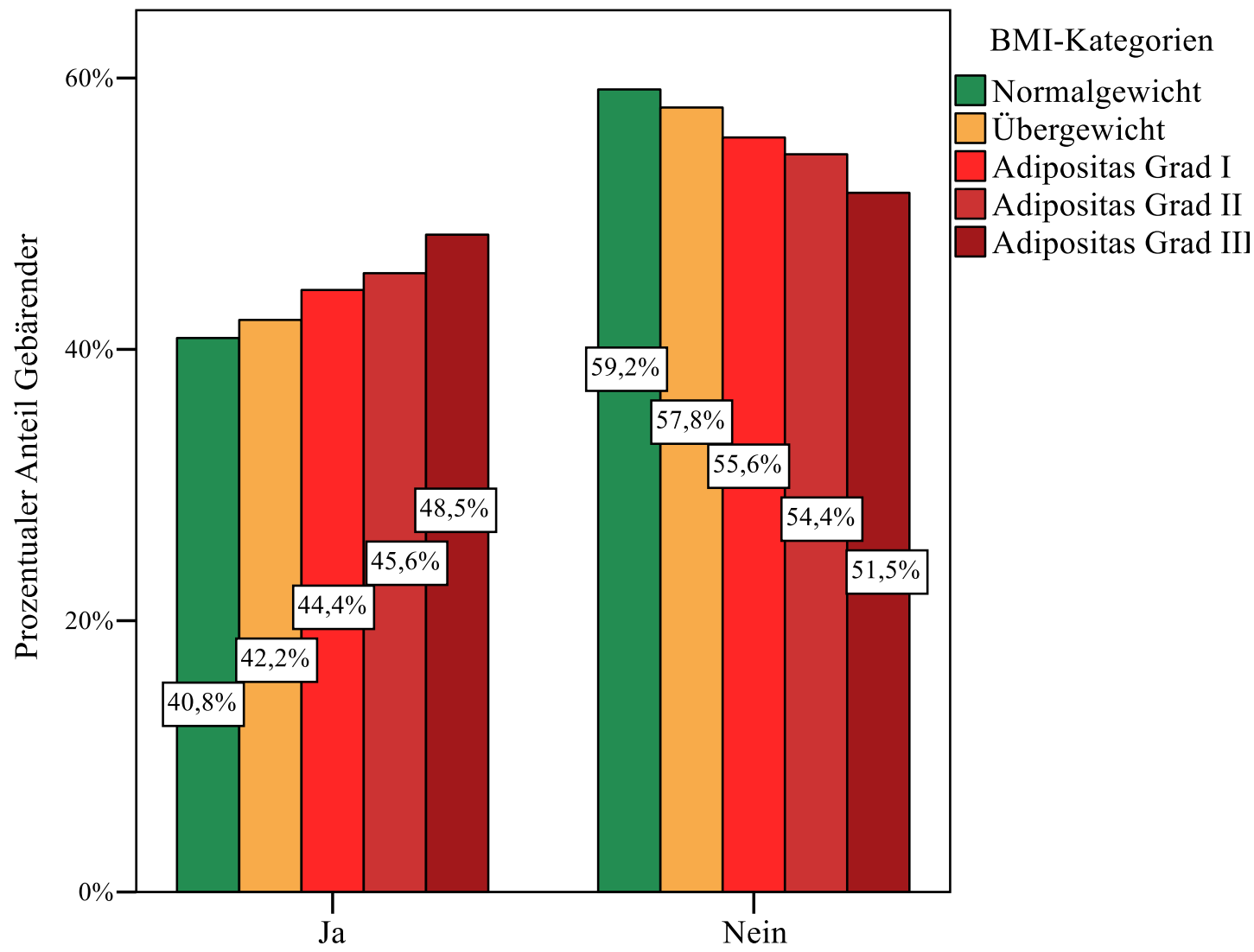

Wehenmittel

Abb. 15 Relative Häufigkeitsverteilung des Einsatzes eines Wehenmittels unter der Geburt in Bezug aufmaternale BMI-Kategorien $(N=24518)$ 


\subsubsection{Schulterdystokie und resultierende kindliche Geburtsverletzungen}

Um die Häufigkeit einer Schulterdystokie in Abhängigkeit vom mütterlichen BMI zu analysieren, wurde die Subgruppe der Frauen mit einer vaginalen Geburt eines bei Geburtsbeginn vitalen Feten aus Schädellage herangezogen $(\mathrm{N}=20858)$.

Insgesamt kam es im Untersuchungszeitraum bei 91 Entbindungen $(0,4 \%)$ zu einer Schulterdystokie, wobei der Anteil unter übergewichtigen (28 Fälle) und adipösen Frauen (14 Fälle) mit jeweils 0,7\% signifikant größer war als unter normalgewichtigen Frauen (49 Fälle) mit $0,3 \%$ ( $\mathrm{p}=0,003$ bzw. $\mathrm{p}=0,020$; Chi-Quadrat-Test).

Die höchste Frequenz einer Schulterdystokie wurde mit 2,0\% (5 Fälle) unter den Frauen der BMI-Kategorie Adipositas Grad III registriert (Tab. 42).

Tab. 42 Absolute und relative Häufigkeiten einer Schulterdystokie in Bezug auf maternale BMI-Kategorien

\begin{tabular}{lccccccc} 
& \multicolumn{7}{c}{ BMI-Kategorien $\left[\mathrm{kg} / \mathrm{m}^{2}\right]$} \\
Schulter- \\
\cline { 2 - 7 } dystokie & $\mathbf{1 8 , 5 - 2 4 , 9}$ & $\mathbf{2 5 , 0 - 2 9 , 9}$ & $\mathbf{3 0 , 0 - 3 4 , 9}$ & $\mathbf{3 5 , 0 - 3 9 , 9}$ & $\mathbf{2 4 0}$ & $\mathbf{2 3 0}$ & Gesamt \\
\hline \hline \multirow{2}{*}{ Ja } & 49 & 28 & 7 & 2 & 5 & 14 & 91 \\
& $0,3 \%$ & $0,7 \%$ & $0,5 \%$ & $0,4 \%$ & $2,0 \%$ & $0,7 \%$ & $0,4 \%$ \\
\hline \multirow{2}{*}{ Nein } & 14512 & 4183 & 1349 & 481 & 242 & 2072 & 20767 \\
& $99,7 \%$ & $99,3 \%$ & $99,5 \%$ & $99,6 \%$ & $98,0 \%$ & $99,3 \%$ & $99,6 \%$ \\
\hline
\end{tabular}

Diese Beobachtungen konnten auch in der Analyse der Subgruppe nach Ausschluss der Frauen mit einer diabetischen Stoffwechsellage, einer vaginal-operativen Entbindung und der Geburt eines als LGA eingestuften Kindes bestätigt werden, was für einen unabhängigen Einfluss der maternalen Adipositas von den genannten Risikofaktoren auf das Schulterdystokie-Risiko sprach (Tab. 43).

Die Schulterdystokie-Rate war auch in dieser Subgruppe von Patientinnen (N=17575) unter übergewichtigen mit 0,7\% (23 Fälle, $p=0,002$ ) und unter adipösen Gebärenden mit $0,8 \%$ (12 Fälle, $\mathrm{p}=0,003$ ) signifikant höher als im Referenzkollektiv mit 0,3\% (37 Fälle), wobei der Anteil der Schulterdystokie-Fälle insgesamt 0,4\% (72 Fälle) betrug. Auch hier diente der ChiQuadrat-Test als bivariate Analysemethode. 
Tab. 43 Absolute und relative Häufigkeiten einer Schulterdystokie in Bezug auf maternale BMI-Kategorien nach Ausschluss der Frauen mit Diabetes mellitus, einer vaginal-operativen Entbindung und der Geburt eines LGAKindes

\begin{tabular}{|c|c|c|c|c|c|c|c|}
\hline \multirow[b]{2}{*}{$\begin{array}{l}\text { Schulter- } \\
\text { dystokie }\end{array}$} & \multicolumn{6}{|c|}{ BMI-Kategorien $\left[\mathrm{kg} / \mathrm{m}^{2}\right]$} & \multirow[b]{2}{*}{$\begin{array}{c}\text { Gesamt } \\
\mathrm{N}=17575\end{array}$} \\
\hline & $\begin{array}{c}\mathbf{1 8 , 5 - 2 4 , 9} \\
\mathrm{n}=12499\end{array}$ & $\begin{array}{c}\mathbf{2 5 , 0 - 2 9 , 9} \\
\mathrm{n}=3497\end{array}$ & $\begin{array}{c}\mathbf{3 0 , 0 - 3 4 , 9} \\
\mathrm{n}=1069\end{array}$ & $\begin{array}{c}\mathbf{3 5 , 0 - 3 9 , 9} \\
\mathrm{n}=366\end{array}$ & $\begin{array}{c}\geq 40 \\
n=162\end{array}$ & $\begin{array}{c}\geq \mathbf{3 0} \\
n=1633\end{array}$ & \\
\hline \multirow{2}{*}{$\mathrm{Ja}$} & 37 & 23 & $\overline{6}$ & 2 & 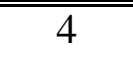 & 12 & 72 \\
\hline & $0,3 \%$ & $0,7 \%$ & $0,6 \%$ & $0,6 \%$ & $2,5 \%$ & $0,8 \%$ & $0,4 \%$ \\
\hline \multirow{2}{*}{ Nein } & 12462 & 3474 & 1063 & 364 & 158 & 1567 & 17503 \\
\hline & $99,7 \%$ & $99,3 \%$ & $99,4 \%$ & $99,5 \%$ & $97,5 \%$ & $99,2 \%$ & $99,6 \%$ \\
\hline
\end{tabular}

Die in Tabelle 44 dargestellte, nach mütterlicher Parität stratifizierte Auswertung der Schulterdystokie-Fälle zeigt, dass das Ereignis unter Pluriparae insgesamt mit 0,6\% (64 Fälle) signifikant häufiger zu finden war als unter Primiparae mit 0,3\% (27 Fälle, p<0,001).

In der Subgruppe der Pluriparae trat unter übergewichtigen mit 0,8\% (20 Fälle) und adipösen Müttern mit 1,0\% (12 Fälle) häufiger eine Schulterdystokie auf als im normalgewichtigen Vergleichskollektiv mit 0,5\% (32 Fälle). Der durchgeführte Chi-Quadrat-Test bestätigte mit $\mathrm{p}=0,028$ bzw. $\mathrm{p}=0,023$ jeweils die statistische Signifikanz.

In der Gruppe der Erstgebärenden konnte im exakten Test nach Fisher kein statistisch signifikanter Zusammenhang zwischen dem mütterlichen BMI und der Schulterdystokie-Rate festgestellt werden, wobei in dieser Stichprobe insgesamt lediglich 27 Fälle verzeichnet wurden. Der Anteil betrug unter übergewichtigen Frauen 0,4\% (8 Fälle) und unter adipösen Frauen 0,2\% (2 Fälle), was sich jeweils nicht signifikant vom Referenzkollektiv mit 0,2\% (17 Fälle) unterschied $(\mathrm{p}=0,129$ bzw. $\mathrm{p}=1,000)$.

Somit war der Einfluss des mütterlichen BMI auf das Schulterdystokie-Risiko in diesem Studienkollektiv abhängig von der Parität der Frau und lediglich in der Gruppe der Pluriparae, nicht jedoch in der Gruppe der Primiparae, signifikant mit mütterlicher Adipositas assoziiert. 
Tab. 44 Absolute und relative Häufigkeiten einer Schulterdystokie in Bezug auf maternale BMI-Kategorien, stratifiziert nach Parität

BMI-Kategorien $\left[\mathrm{kg} / \mathrm{m}^{2}\right]$

\begin{tabular}{|c|c|c|c|c|c|c|c|}
\hline $\begin{array}{l}\text { Schulter- } \\
\text { dystokie }\end{array}$ & $\begin{array}{c}\mathbf{1 8 , 5 - 2 4 , 9} \\
n=14561\end{array}$ & $\begin{array}{c}\mathbf{2 5 , 0 - 2 9 , 9} \\
n=4211\end{array}$ & $\begin{array}{c}\mathbf{3 0 , 0 - 3 4 , 9} \\
\mathrm{n}=1356\end{array}$ & $\begin{array}{c}35,0-39,9 \\
n=483\end{array}$ & $\begin{array}{c}\geq \mathbf{4 0} \\
n=247\end{array}$ & $\begin{array}{c}\geq \mathbf{3 0} \\
n=2086\end{array}$ & $\begin{array}{c}\text { Gesamt } \\
\mathrm{N}=20858\end{array}$ \\
\hline Primipara & $n=7510$ & $\mathrm{n}=1826$ & $\mathrm{n}=551$ & $\mathrm{n}=188$ & $n=100$ & $\mathrm{n}=839$ & $\mathrm{n}=10175$ \\
\hline \multirow{2}{*}{$\mathrm{Ja}$} & 17 & 8 & 2 & $\overline{0}$ & 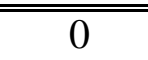 & 2 & 27 \\
\hline & $0,2 \%$ & $0,4 \%$ & $0,4 \%$ & $0,0 \%$ & $0,0 \%$ & $0,2 \%$ & $0,3 \%$ \\
\hline \multirow{2}{*}{ Nein } & 7493 & 1818 & 549 & 188 & 100 & 837 & 10148 \\
\hline & $99,8 \%$ & $99,6 \%$ & $99,6 \%$ & $100,0 \%$ & $100,0 \%$ & $99,8 \%$ & $99,7 \%$ \\
\hline Pluripara & $\mathrm{n}=7051$ & $\mathrm{n}=2385$ & $\mathrm{n}=805$ & $\mathrm{n}=295$ & $\mathrm{n}=147$ & $\mathrm{n}=1247$ & 10683 \\
\hline \multirow{2}{*}{$\mathrm{Ja}$} & 32 & 20 & $\overline{5}$ & 2 & $\overline{5}$ & 12 & 64 \\
\hline & $0,5 \%$ & $0,8 \%$ & $0,6 \%$ & $0,7 \%$ & $3,4 \%$ & $1,0 \%$ & $0,6 \%$ \\
\hline \multirow{2}{*}{ Nein } & 7019 & 2365 & 800 & 293 & 142 & 1235 & 10619 \\
\hline & $99,5 \%$ & $99,2 \%$ & $99,4 \%$ & $99,3 \%$ & $96,6 \%$ & $99,0 \%$ & $99,4 \%$ \\
\hline
\end{tabular}

In vier von insgesamt 91 Fällen einer Schulterdystokie $(4,4 \%)$ kam es infolgedessen zu einer kindlichen Geburtsverletzung.

Bei der Geburt von drei normalgewichtigen Frauen wurden zwei Fälle einer Verletzung des Plexus brachialis beschrieben, wobei das kindliche Geburtsgewicht $4425 \mathrm{~g}$ bzw. $3860 \mathrm{~g}$ betrug sowie ein Fall einer Klavikulafraktur bei einem kindlichen Gewicht von 4400 g. Bei der Entbindung einer übergewichtigen Frau trat eine kindliche Erb-Lähmung auf, wobei das Geburtsgewicht $4230 \mathrm{~g}$ betrug.

Aufgrund der geringen Fallzahl war die statistische Auswertung hinsichtlich eines eventuellen signifikanten Zusammenhangs mit der BMI-Kategorie der Mutter nicht möglich.

Tabelle 45 zeigt ausgewählte mütterliche und kindliche Charakteristika der beschriebenen Fälle. 
Tabelle 45 Mütterliche bzw. kindliche Charakteristika der verschiedenen Fälle einer kindlichen Geburtsverletzung infolge einer Schulterdystokie

Mütterliche bzw. kindliche Charakterisitka

\begin{tabular}{lcccccc}
\cline { 2 - 6 } $\begin{array}{l}\text { Geburts- } \\
\text { verletzung }\end{array}$ & $\begin{array}{c}\text { BMI } \\
{\left[\mathrm{kg} / \mathrm{m}^{2}\right]}\end{array}$ & Parität & $\begin{array}{c}\text { Diabetes } \\
\text { mellitus }\end{array}$ & $\begin{array}{c}\text { Geburts- } \\
\text { modus }\end{array}$ & $\begin{array}{c}\text { Kindliches } \\
\text { Gewicht }[\mathrm{g}]\end{array}$ & $\begin{array}{c}\text { Gestations- } \\
\text { alter [SSW] }\end{array}$ \\
\hline \hline $\begin{array}{l}\text { Plexus } \\
\text { brachialis }\end{array}$ & 21,5 & Primipara & Nein & Vaginal-operativ & 4425 & $41+0$ \\
\hline $\begin{array}{l}\text { Plexus } \\
\text { brachialis }\end{array}$ & 23,4 & Pluripara & Nein & $\begin{array}{c}\text { Spontan vaginal } \\
\text { aus SL }\end{array}$ & 3860 & $39+4$ \\
\hline $\begin{array}{l}\text { Erb- } \\
\text { Lähmung }\end{array}$ & 25,8 & Pluripara & Nein & $\begin{array}{c}\text { Spontan vaginal } \\
\text { aus SL }\end{array}$ & 4230 & $38+3$ \\
\hline $\begin{array}{l}\text { Klavikula- } \\
\text { fraktur }\end{array}$ & 24,8 & Pluripara & Nein & $\begin{array}{c}\text { Spontan vaginal } \\
\text { aus SL }\end{array}$ & 4400 & $40+5$ \\
\hline
\end{tabular}

\subsubsection{Grünes Fruchtwasser}

Die Prävalenz von grünem Fruchtwasser war signifikant mit dem mütterlichen BMI assoziiert, wobei es insgesamt bei 9,0\% der Geburten (2495 Fälle) beobachtet wurde. Bei der Entbindung von übergewichtigen und adipösen Frauen war mekoniumhaltiges Fruchtwasser mit 9,9\% bzw. 11,3\% im Vergleich zur Referenzgruppe mit 8,3\% häufiger zu finden, was der durchgeführte Chi-Quadrat-Test als statistisch signifikant (jeweils p<0,001) bestätigte (Tab. 46; Abb. 16).

Tab. 46 Absolute und relative Häufigkeiten von grünem Fruchtwasser in Bezug auf maternale BMI-Kategorien

BMI-Kategorien $\left[\mathrm{kg} / \mathrm{m}^{2}\right]$

\begin{tabular}{|c|c|c|c|c|c|c|c|}
\hline $\begin{array}{l}\text { Grünes } \\
\text { Fruchtwasser }\end{array}$ & $\begin{array}{c}\mathbf{1 8 , 5 - 2 4 , 9} \\
\mathrm{n}=18809\end{array}$ & $\begin{array}{c}\mathbf{2 5 , 0 - 2 9 , 9} \\
n=5741\end{array}$ & $\begin{array}{c}\mathbf{3 0 , 0 - 3 4 , 9} \\
n=2009\end{array}$ & $\begin{array}{c}\text { 35,0-39,9 } \\
n=774\end{array}$ & $\begin{array}{c}\geq \mathbf{4 0} \\
\mathrm{n}=421\end{array}$ & $\begin{array}{c}\geq \mathbf{3 0} \\
\mathrm{n}=3204\end{array}$ & $\begin{array}{c}\text { Gesamt } \\
\mathrm{N}=27754\end{array}$ \\
\hline \multirow{2}{*}{$\mathrm{Ja}$} & 15664 & 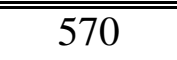 & 225 & $\overline{833}$ & $\overline{533}$ & 361 & 2495 \\
\hline & $8,3 \%$ & $9,9 \%$ & $11,2 \%$ & $10,7 \%$ & $12,6 \%$ & $11,3 \%$ & $9,0 \%$ \\
\hline \multirow{2}{*}{ Nein } & 17245 & 5171 & 1784 & 691 & 368 & 2843 & 25259 \\
\hline & $91,7 \%$ & $90,1 \%$ & $88,8 \%$ & $89,3 \%$ & $87,4 \%$ & $88,7 \%$ & $91,0 \%$ \\
\hline
\end{tabular}




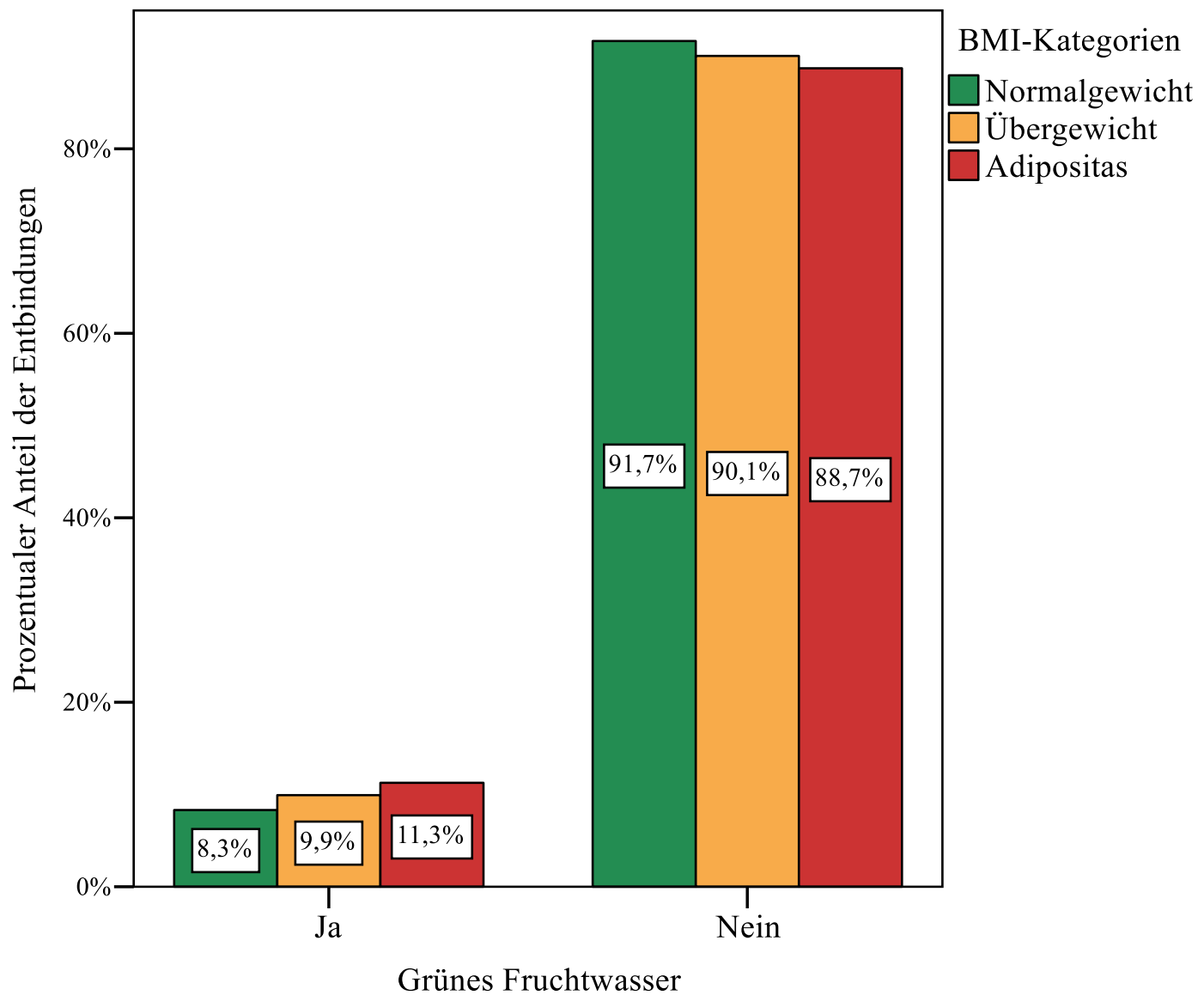

Abb. 16 Relative Häufigkeitsverteilung von grünem Fruchtwasser in Bezug auf maternale BMI-Kategorien $(N=27754)$

\subsubsection{Dauer der Geburt}

Insgesamt betrug die mittlere Dauer der vaginalen Entbindung aus Schädellage unter Primiparae 8,8 Stunden (SD 6,2) und unter Pluriparae 5,5 Stunden (SD 4,8).

Bei der Betrachtung der Geburtsdauer in Bezug auf die mütterliche BMI-Kategorie fiel auf, dass sowohl unter erstgebärenden als auch unter mehrgebärenden adipösen Frauen die mittlere Geburtsdauer mit 9,1 (SD 6,4) bzw. 5,9 Stunden (SD 5,8) länger war, als bei normalgewichtigen Primiparae und Pluriparae mit 8,7 (SD 6,1) bzw. 5,4 Stunden (SD 4,7) (Tab. 47 und 48).

Die Unterschiede zwischen den BMI-Gruppen erwiesen sich mithilfe der einfaktoriellen Varianzanalyse in der Gruppe der Mehrgebärenden als statistisch signifikant ( $\mathrm{p}=0,001)$, was in der Gruppe der Erstgebärenden nicht bestätigt werden konnte $(\mathrm{p}=0,151)$. 
Tab. 47 Mittlere Geburtsdauer und SD der vaginalen Entbindung aus SL unter Primiparae in Bezug aufmaternale BMI-Kategorien

\begin{tabular}{lcccccccc} 
& \multicolumn{7}{c}{ BMI-Kategorien $\left[\mathrm{kg} / \mathrm{m}^{2}\right]$} \\
Geburtsdauer \\
\cline { 2 - 7 } Primipara [h] & $\mathbf{1 8 , 5 - 2 4 , 9}$ & $\begin{array}{c}\mathbf{2 5 , 0 - 2 9 , 9} \\
\mathrm{n}=6248\end{array}$ & $\begin{array}{c}\mathbf{3 0 , 0 - 3 4 , 9} \\
\mathrm{n}=1528\end{array}$ & $\begin{array}{c}\mathbf{3 5 , 0 - 3 9 , 9} \\
\mathrm{n}=467\end{array}$ & $\begin{array}{c}\mathbf{2 4 0} \\
\mathrm{n}=76\end{array}$ & $\begin{array}{c}\mathbf{2 3 0} \\
\mathrm{n}=705\end{array}$ & $\begin{array}{c}\text { Gesamt } \\
\mathrm{N}=8481\end{array}$ \\
\hline \hline Mittelwert & 8,7 & 9,1 & 9,2 & 9,0 & 8,7 & 9,1 & 8,8 \\
\hline SD & 6,1 & 6,1 & 6,5 & 6,0 & 6,1 & 6,4 & 6,2 \\
\hline
\end{tabular}

Tab. 48 Mittlere Geburtsdauer und SD der vaginalen Entbindung aus SL unter Pluriparae in Bezug auf maternale BMI-Kategorien

\begin{tabular}{|c|c|c|c|c|c|c|c|}
\hline \multirow{3}{*}{$\begin{array}{l}\text { Geburtsdauer } \\
\text { Pluripara [h] } \\
\end{array}$} & \multicolumn{6}{|c|}{ BMI-Kategorien $\left[\mathrm{kg} / \mathrm{m}^{2}\right]$} & \multirow{3}{*}{$\begin{array}{c}\text { Gesamt } \\
\mathrm{N}=10101 \\
\end{array}$} \\
\hline & $18,5-24,9$ & $25,0-29,9$ & $30,0-34,9$ & $35,0-39,9$ & $\geq 40$ & $\geq \mathbf{3 0}$ & \\
\hline & $n=6667$ & $\mathrm{n}=2239$ & $\mathrm{n}=775$ & $\mathrm{n}=282$ & $\mathrm{n}=137$ & $\mathrm{n}=1194$ & \\
\hline Mittelwert & 5,4 & 5,5 & 5,8 & 5,8 & 6,6 & 5,9 & 5,5 \\
\hline SD & 4,7 & 4,5 & 5,4 & 6,0 & 7,6 & 5,8 & 4,8 \\
\hline
\end{tabular}

Die Auswertungen hinsichtlich eines protrahierten Verlaufs bzw. eines Stillstands der Geburt sind jeweils für die Eröffnungsphase in Tabellen 49 und 50 und für die Austreibungsphase in Tabellen 51 und 52 dargestellt. Für diese Analysen waren die Patientinnen, die primär sectioniert wurden, vom untersuchten Studienkollektiv ausgeschlossen ( $\mathrm{N}=24518)$.

Die Prävalenz einer protrahierten EP betrug insgesamt 1,4\%, wobei der prozentuale Anteil bei adipösen Frauen mit 1,8\% größer war als bei normalgewichtigen Frauen mit 1,4\%, was sich im Chi-Quadrat-Test als nicht statistisch signifikant erwies $(\mathrm{p}=0,112)$.

Die Frequenz eines Geburtsstillstands in der EP betrug insgesamt 3,0\% und stieg mit zunehmender BMI-Kategorie der Frau von Normalgewicht (2,6\%) bis Adipositas Grad III (7,1\%) an. Der Unterschied war im Chi-Quadrat-Test für übergewichtige mit 3,3\% und adipöse Frauen mit 5,0\% im Vergleich zur Referenzgruppe mit 2,6\% statistisch signifikant ( $\mathrm{p}=0,006$ bzw. $\mathrm{p}<0,001)$. 
Tab. 49 Absolute und relative Häufigkeiten einer protrahierten EP in Bezug auf maternale BMI-Kategorien

BMI-Kategorien $\left[\mathrm{kg} / \mathrm{m}^{2}\right]$

\begin{tabular}{|c|c|c|c|c|c|c|c|}
\hline $\begin{array}{l}\text { protrahierte } \\
\text { EP }\end{array}$ & $\begin{array}{c}\mathbf{1 8 , 5 - 2 4 , 9} \\
\mathrm{n}=16896\end{array}$ & $\begin{array}{c}\mathbf{2 5 , 0 - 2 9 , 9} \\
n=5001\end{array}$ & $\begin{array}{c}\mathbf{3 0 , 0 - 3 4 , 9} \\
n=1681\end{array}$ & $\begin{array}{c}\mathbf{3 5 , 0 - 3 9 , 9} \\
n=616\end{array}$ & $\begin{array}{c}\geq \mathbf{4 0} \\
\mathrm{n}=324\end{array}$ & $\begin{array}{c}\geq \mathbf{3 0} \\
\mathrm{n}=2621\end{array}$ & $\begin{array}{c}\text { Gesamt } \\
\mathrm{N}=24518\end{array}$ \\
\hline \multirow{2}{*}{$\mathrm{Ja}$} & 230 & "67 & $\overline{311}$ & $\overline{99}$ & 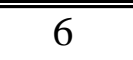 & $\bar{~} 46$ & 343 \\
\hline & $1,4 \%$ & $1,3 \%$ & $1,8 \%$ & $1,5 \%$ & $1,9 \%$ & $1,8 \%$ & $1,4 \%$ \\
\hline \multirow{2}{*}{ Nein } & 16666 & 4934 & 1650 & 607 & 318 & 2575 & 24175 \\
\hline & $98,6 \%$ & $98,7 \%$ & $98,2 \%$ & $98,5 \%$ & $98,1 \%$ & $98,2 \%$ & $98,6 \%$ \\
\hline
\end{tabular}

Tab. 50 Absolute und relative Häufigkeiten eines Geburtsstillstands in der EP in Bezug auf maternale BMI-Kategorien

BMI-Kategorien $\left[\mathrm{kg} / \mathrm{m}^{2}\right]$

\begin{tabular}{|c|c|c|c|c|c|c|c|}
\hline $\begin{array}{l}\text { Geburts- } \\
\text { stillstand EP }\end{array}$ & $\begin{array}{c}\mathbf{1 8 , 5 - 2 4 , 9} \\
n=16896\end{array}$ & $\begin{array}{c}\mathbf{2 5 , 0 - 2 9 , 9} \\
n=5001\end{array}$ & $\begin{array}{c}\mathbf{3 0 , 0 - 3 4 , 9} \\
\mathrm{n}=1681\end{array}$ & $\begin{array}{c}\mathbf{3 5 , 0 - 3 9 , 9} \\
n=616\end{array}$ & $\begin{array}{c}\geq 40 \\
n=324\end{array}$ & $\begin{array}{c}\geq \mathbf{3 0} \\
n=2621\end{array}$ & $\begin{array}{c}\text { Gesamt } \\
\mathrm{N}=24518\end{array}$ \\
\hline \multirow{2}{*}{$\mathrm{Ja}$} & 439 & 166 & 77 & 30 & 23 & 130 & 735 \\
\hline & $2,6 \%$ & $3,3 \%$ & $4,6 \%$ & $4,9 \%$ & $7,1 \%$ & $5,0 \%$ & $3,0 \%$ \\
\hline \multirow{2}{*}{ Nein } & 16457 & 4835 & 1604 & 586 & 301 & 2491 & 23783 \\
\hline & $97,4 \%$ & $96,7 \%$ & $95,4 \%$ & $95,1 \%$ & $92,9 \%$ & $95,0 \%$ & $97,0 \%$ \\
\hline
\end{tabular}

Bei den adipösen Patientinnen kam es dagegen mit 1,8\% signifikant seltener zu einem protrahierten Verlauf der AP als bei normalgewichtigen Frauen mit 3,0\% (p=0,001; Chi-QuadratTest), wobei die AP insgesamt bei 2,8\% der Geburten als protrahiert eingestuft wurde.

Auch der Geburtsstillstand in der AP trat im Vergleich zur Referenzgruppe (4,4\%) bei fettleibigen Gebärenden (4,2\%) prozentual seltener auf. Die Unterschiede zwischen den Gruppen zeigten sich im Chi-Quadrat-Test als nicht statistisch signifikant $(\mathrm{p}=0,596)$.

Tab. 51 Absolute und relative Häufigkeiten einer protrahierten AP in Bezug auf maternale BMI-Kategorien

BMI-Kategorien $\left[\mathrm{kg} / \mathrm{m}^{2}\right]$

\begin{tabular}{lccccccc} 
protrahierter & $\mathbf{1 8 , 5 - 2 4 , 9}$ & $\mathbf{2 5 , 0 - 2 9 , 9}$ & $\mathbf{3 0 , 0 - 3 4 , 9}$ & $\mathbf{3 5 , 0 - 3 9 , 9}$ & $\mathbf{2 4 0}$ & $\mathbf{2 3 0}$ & Gesamt \\
Verlauf AP & $\mathrm{n}=16896$ & $\mathrm{n}=5001$ & $\mathrm{n}=1681$ & $\mathrm{n}=616$ & $\mathrm{n}=324$ & $\mathrm{n}=2621$ & $\mathrm{~N}=24518$ \\
\hline \hline \multirow{2}{*}{ Ja } & 503 & 136 & 29 & 11 & 8 & 48 & 687 \\
& $3,0 \%$ & $2,7 \%$ & $1,7 \%$ & $1,8 \%$ & $2,5 \%$ & $1,8 \%$ & $2,8 \%$ \\
\hline \multirow{2}{*}{ Nein } & 16393 & 4865 & 1652 & 605 & 316 & 2573 & 23831 \\
& $97,0 \%$ & $97,3 \%$ & $98,3 \%$ & $98,2 \%$ & $97,5 \%$ & $98,2 \%$ & $97,2 \%$ \\
\hline
\end{tabular}


Tab. 52 Absolute und relative Häufigkeiten eines Geburtsstillstands in der EP in Bezug auf maternale BMI-Kategorien

\begin{tabular}{lccccccc} 
& \multicolumn{7}{c}{ BMI-Kategorien $\left[\mathrm{kg} / \mathrm{m}^{2}\right]$} \\
Geburts- & $\mathbf{1 8 , 5 - 2 4 , 9}$ & $\mathbf{2 5 , 0 - 2 9 , 9}$ & $\mathbf{3 0 , 0 - 3 4 , 9}$ & $\mathbf{3 5 , 0 - 3 9 , 9}$ & $\mathbf{2 4 0}$ & $\geq \mathbf{3 0}$ & Gesamt \\
stillstand AP & $\mathrm{n}=16896$ & $\mathrm{n}=5001$ & $\mathrm{n}=1681$ & $\mathrm{n}=616$ & $\mathrm{n}=324$ & $\mathrm{n}=2621$ & $\mathrm{~N}=24518$ \\
\hline \hline \multirow{2}{*}{ Ja } & 741 & 204 & 72 & 23 & 14 & 109 & 1054 \\
& $4,4 \%$ & $4,1 \%$ & $4,3 \%$ & $3,7 \%$ & $4,3 \%$ & $4,2 \%$ & $4,3 \%$ \\
\hline \multirow{2}{*}{ Nein } & 16155 & 4797 & 1609 & 593 & 310 & 2512 & 23464 \\
& $95,6 \%$ & $95,9 \%$ & $95,7 \%$ & $96,3 \%$ & $95,7 \%$ & $95,8 \%$ & $95,7 \%$ \\
\hline
\end{tabular}

\subsubsection{Blutverlust der Mutter}

Der vom Geburtshelfer geschätzte mittlere Blutverlust der Frau unter der Geburt stieg mit zunehmendem maternalen BMI von 398,9 ml (SD 238,5) in der BMI-Gruppe Normalgewicht auf einen Mittelwert von 444,0 ml (SD 207,1) unter den Müttern der Kategorie Adipositas Grad III an (Tab. 53).

Die ANOVA zeigte einen signifikanten Unterschied zwischen den BMI-Gruppen $(p<0,001)$. Übergewichtige $(p=0,072)$ und adipöse Gebärende $(p<0,001)$ verloren im Mittel mit 405,4 ml (SD 240,5) bzw. 426,6 ml (SD 229,2) mehr Blut, als normalgewichtige Patientinnen mit 398,9 ml (SD 238,5). Der Unterschied zwischen den BMI-Gruppen Normalgewicht und Adipositas stellte sich in den paarweisen Mehrfachvergleichen als statistisch signifikant heraus.

Tab. 53 Mittelwert und SD des Blutverlustes der Mütter unter der Geburt in Bezug auf deren BMI-Kategorien

\begin{tabular}{lccccccc} 
& \multicolumn{7}{c}{ BMI-Kategorien $\left[\mathrm{kg} / \mathrm{m}^{2}\right]$} \\
\cline { 2 - 7 } $\begin{array}{l}\text { Blut- } \\
\text { verlust [ml] }\end{array}$ & $\begin{array}{c}\mathbf{1 8 , 5 - 2 4 , 9} \\
\mathrm{n}=18656\end{array}$ & $\begin{array}{c}\mathbf{2 5 , 0 - 2 9 , 9} \\
\mathrm{n}=5682\end{array}$ & $\begin{array}{c}\mathbf{3 0 , 0 - 3 4 , 9} \\
\mathrm{n}=1996\end{array}$ & $\begin{array}{c}\mathbf{3 5 , 0 - 3 9 , 9} \\
\mathrm{n}=767\end{array}$ & $\begin{array}{c}\mathbf{2 4 0} \\
\mathrm{n}=413\end{array}$ & $\begin{array}{c}\mathbf{2 3 0} \\
\mathrm{n}=3176\end{array}$ & $\begin{array}{c}\text { Gesamt } \\
\mathrm{N}=27514\end{array}$ \\
\hline \hline Mittelwert & 398,9 & 405,4 & 420,4 & 433,4 & 444,0 & 426,6 & 403,4 \\
\hline SD & 238,5 & 240,5 & 242,7 & 202,5 & 207,1 & 229,2 & 237,9
\end{tabular}

Im Hinblick auf eine peripartale Hämorrhagie konnten im Chi-Quadrat-Test keine signifikanten Unterschiede zwischen den BMI-Kategorien nachgewiesen werden. Bei vaginalem Entbindungsmodus waren insgesamt 6,7\% der Frauen (1412 Fälle) von einer PPH betroffen, bei Entbindung per Sectio caesarea betrug der Anteil mit 93 Fällen 1,4\% (Tab. 54 und 55). 
Nach vaginaler Geburt kam es bei übergewichtigen Frauen mit 6,2\% (p=0,183) etwas seltener $\mathrm{zu}$ einem Blutverlust von mehr als $500 \mathrm{ml}$, als bei normalgewichtigen Frauen mit 6,8\%, bei adipösen Frauen dagegen etwas häufiger mit 7,4\% ( $\mathrm{p}=0,274)$.

Unter den Frauen, die per Sectio caesarea entbanden, war der Anteil derer mit einem Blutverlust von mehr als $1000 \mathrm{ml}$ unter übergewichtigen mit $1,4 \%(\mathrm{p}=0,671)$ und adipösen Patientinnen mit $0,8 \%(p=0,065)$ kleiner als unter Frauen der Referenzgruppe mit 1,6\%.

Tab. 54 Absolute und relative Häufigkeiten einer PPH nach vaginaler Geburt in Bezug auf maternale BMI-Kategorien

BMI-Kategorien $\left[\mathrm{kg} / \mathrm{m}^{2}\right]$

\begin{tabular}{|c|c|c|c|c|c|c|c|}
\hline $\begin{array}{l}\text { PPH vaginale } \\
\text { Entbindung } \\
\end{array}$ & $\begin{array}{c}\mathbf{1 8 , 5 - 2 4 , 9} \\
\mathrm{n}=14711 \\
\end{array}$ & $\begin{array}{c}\mathbf{2 5 , 0 - 2 9 , 9} \\
\mathrm{n}=4220 \\
\end{array}$ & $\begin{array}{c}\mathbf{3 0 , 0 - 3 4 , 9} \\
\mathrm{n}=1372 \\
\end{array}$ & $\begin{array}{c}\mathbf{3 5 , 0 - 3 9 , 9} \\
\mathrm{n}=485 \\
\end{array}$ & $\begin{array}{c}\geq \mathbf{4 0} \\
\mathrm{n}=249\end{array}$ & $\begin{array}{c}\geq \mathbf{3 0} \\
n=2106\end{array}$ & $\begin{array}{c}\text { Gesamt } \\
\mathrm{N}=21037 \\
\end{array}$ \\
\hline \multirow{2}{*}{$\mathrm{Ja}$} & 995 & 261 & 95 & 44 & 17 & 156 & 1412 \\
\hline & $6,8 \%$ & $6,2 \%$ & $6,9 \%$ & $9,1 \%$ & $6,8 \%$ & $7,4 \%$ & $6,7 \%$ \\
\hline \multirow{2}{*}{ Nein } & 13716 & 3959 & 1277 & 441 & 232 & 1950 & 19625 \\
\hline & $93,2 \%$ & $93,8 \%$ & $93,1 \%$ & $90,9 \%$ & $93,2 \%$ & $92,6 \%$ & $93,3 \%$ \\
\hline
\end{tabular}

Tab. 55 Absolute und relative Häufigkeiten einer PPH nach Sectio caesarea in Bezug auf maternale BMI-Kategorien

BMI-Kategorien $\left[\mathrm{kg} / \mathrm{m}^{2}\right]$

\begin{tabular}{|c|c|c|c|c|c|c|c|}
\hline $\begin{array}{l}\text { PPH Sectio } \\
\text { caesarea }\end{array}$ & $\begin{array}{c}\mathbf{1 8 , 5 - 2 4 , 9} \\
n=3944\end{array}$ & $\begin{array}{c}\mathbf{2 5 , 0 - 2 9 , 9} \\
n=1462\end{array}$ & $\begin{array}{c}\mathbf{3 0 , 0 - 3 4 , 9} \\
\mathrm{n}=624\end{array}$ & $\begin{array}{c}\mathbf{3 5 , 0 - 3 9 , 9} \\
\mathrm{n}=282\end{array}$ & $\begin{array}{c}\geq 40 \\
n=164\end{array}$ & $\begin{array}{c}\geq \mathbf{3 0} \\
n=1070\end{array}$ & $\begin{array}{r}\text { Gesamt } \\
\mathrm{N}=6476\end{array}$ \\
\hline \multirow{2}{*}{$\mathrm{Ja}$} & 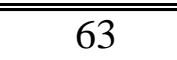 & 21 & 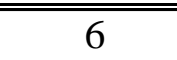 & $\bar{~} 1$ & $\overline{2}$ & $\bar{~} 9$ & $\bar{~} 93$ \\
\hline & $1,6 \%$ & $1,4 \%$ & $1,0 \%$ & $0,4 \%$ & $1,2 \%$ & $0,8 \%$ & $1,4 \%$ \\
\hline Nein & $\begin{array}{c}3881 \\
98,4 \%\end{array}$ & $\begin{array}{c}1441 \\
98,6 \%\end{array}$ & $\begin{array}{c}618 \\
99,0 \%\end{array}$ & $\begin{array}{c}281 \\
99,6 \%\end{array}$ & $\begin{array}{c}162 \\
98,8 \%\end{array}$ & $\begin{array}{c}1061 \\
99,2 \%\end{array}$ & $\begin{array}{c}6383 \\
98,6 \%\end{array}$ \\
\hline
\end{tabular}




\subsection{Kindliches Outcome}

\subsubsection{Geburtsgewicht}

Das mittlere Geburtsgewicht der Kinder normalgewichtiger Frauen betrug 3260,2 g (SD 621,5) mit einer Spannbreite von $300 \mathrm{~g}$ bis $5390 \mathrm{~g}$. Im Vergleich dazu waren die Neugeborenen von übergewichtigen und adipösen Frauen im Mittel mit 3348,3 g (SD 664,7) bzw. 3338,7 g (SD 720,0) schwerer, wobei das Gewicht zwischen $320 \mathrm{~g}$ und $5700 \mathrm{~g}$ bzw. $445 \mathrm{~g}$ und $5410 \mathrm{~g}$ schwankte (Tab. 56; Abb. 17).

Die Unterschiede zwischen den BMI-Gruppen zeigten sich in der einfaktorielle Varianzanalyse bzw. den paarweisen Mehrfachvergleichen mit jeweils $p<0,001$ als statistisch signifikant.

Tab. 56 Mittelwert und SD des kindlichen Geburtsgewichts in Bezug auf maternale BMI-Kategorien

\begin{tabular}{lccccccc} 
& \multicolumn{7}{c}{ BMI-Kategorien $\left[\mathrm{kg} / \mathrm{m}^{2}\right]$} \\
\cline { 2 - 6 } $\begin{array}{l}\text { Geburts- } \\
\text { gewicht [g] }\end{array}$ & $\begin{array}{c}\mathbf{1 8 , 5 - 2 4 , 9} \\
\mathrm{n}=18805\end{array}$ & $\begin{array}{c}\mathbf{2 5 , 0 - 2 9 , 9} \\
\mathrm{n}=5738\end{array}$ & $\begin{array}{c}\mathbf{3 0 , 0 - 3 4 , 9} \\
\mathrm{n}=2008\end{array}$ & $\begin{array}{c}\mathbf{3 5 , 0 - 3 9 , 9} \\
\mathrm{n}=774\end{array}$ & $\begin{array}{c}\mathbf{2} \mathbf{4 0} \\
\mathrm{n}=420\end{array}$ & $\begin{array}{c}\mathbf{2} \mathbf{3 0} \\
\mathrm{n}=3202\end{array}$ & $\begin{array}{c}\text { Gesamt } \\
\mathrm{N}=27745\end{array}$ \\
\hline \hline Mittelwert & 3260,2 & 3348,3 & 3326,2 & 3355,3 & 3367,3 & 3338,7 & 3287,5 \\
\hline SD & 621,5 & 664,7 & 698,5 & 721,5 & 813,3 & 720,0 & 643,8 \\
\hline Minimum & 300 & 320 & 450 & 490 & 445 & 445 & \\
\hline Maximum & 5390 & 5700 & 5210 & 5410 & 5070 & 5410 & \\
\hline
\end{tabular}




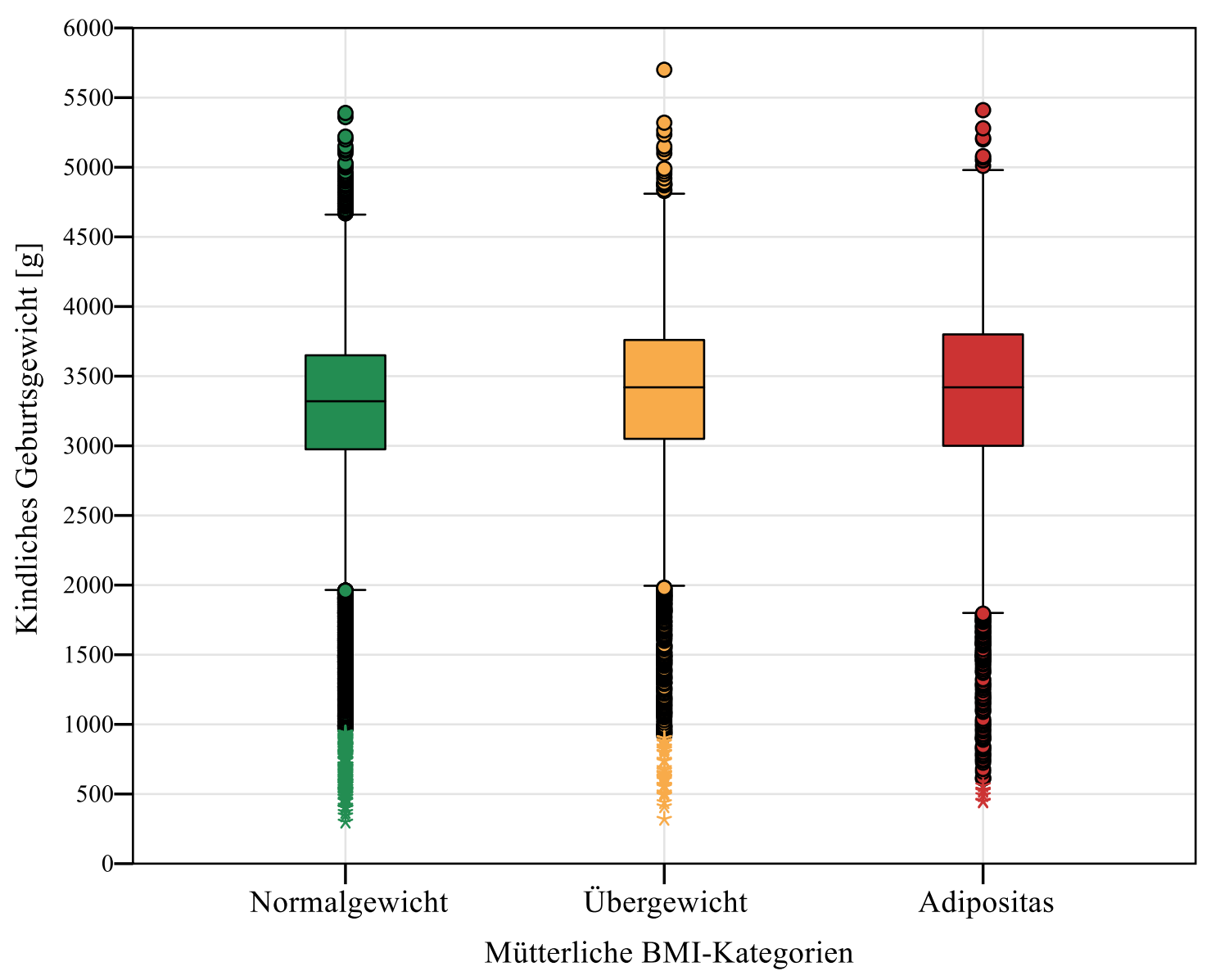

Abb. 17 Kindliches Geburtsgewicht [g] in Bezug auf maternale BMI-Kategorien $(N=27745)$

Zudem wurde die Höhe der kindlichen Gewichtsperzentile, klassifiziert in LGA (>90. Perzentile) und SGA $(<10$. Perzentile), in Bezug auf die maternale BMI-Kategorie analysiert.

Im Untersuchungszeitraum wurden insgesamt 2749 Kinder (9,9\%) als LGA eingeordnet, wobei eine signifikante Assoziation mit dem BMI der Frau festzustellen war. Die Neugeborenen von übergewichtigen und adipösen Patientinnen wurden mit 13,1\% bzw. 16,5\% signifikant häufiger als LGA eingestuft als die Kinder normalgewichtiger Frauen mit 7,8\% (jeweils p <0,001; ChiQuadrat-Test). Es konnte weiterhin beobachtet werden, dass der Anteil der Frauen, deren Kinder als LGA klassifiziert wurden, mit der BMI-Kategorie anstieg und unter Müttern der Kategorie Adipositas Grad III einen Wert von 22,2\% erreichte (Tab. 57; Abb. 18).

Auch die Prävalenz von SGA-Kindern erwies sich in dieser Studie als signifikant mit dem mütterlichen BMI assoziiert, wobei insgesamt 2571 Fälle auftraten (9,3\%). Die Kinder von übergewichtigen und adipösen Müttern in ihrer Gesamtheit wurden mit jeweils 8,2\% signifikant seltener als SGA eingestuft als die Neugeborenen der Frauen des Referenzkollektivs mit 9,8\% ( $\mathrm{p}<0,001$ bzw. $\mathrm{p}=0,004$; Chi-Quadrat-Test). 
Dabei fiel auf, dass der Anteil der Patientinnen mit als SGA klassifizierten Kindern in der Gruppe Adipositas Grad I mit 7,5\% am niedrigsten war, bei Frauen mit Adipositas Grad II auf 8,8\% anstieg und unter Frauen mit Adipositas Grad III mit 9,6\% fast gleich groß war wie unter den Schwangeren mit Normalgewicht (Tab. 57; Abb. 18).

Tab. 57 Absolute und relative Häufigkeiten der klassifizierten kindlichen Geburtsgewichtsperzentile in Bezug auf maternale BMI-Kategorien

BMI-Kategorien $\left[\mathrm{kg} / \mathrm{m}^{2}\right]$

\begin{tabular}{|c|c|c|c|c|c|c|c|}
\hline $\begin{array}{l}\text { Gewichts- } \\
\text { perzentile }\end{array}$ & $\begin{array}{c}\mathbf{1 8 , 5 - 2 4 , 9} \\
\mathrm{n}=18771\end{array}$ & $\begin{array}{c}\mathbf{2 5 , 0 - 2 9 , 9} \\
n=5733\end{array}$ & $\begin{array}{c}\mathbf{3 0 , 0 - 3 4 , 9} \\
\mathrm{n}=2005\end{array}$ & $\begin{array}{c}\mathbf{3 5 , 0 - 3 9 , 9} \\
n=773\end{array}$ & $\begin{array}{c}\geq \mathbf{4 0} \\
n=418\end{array}$ & $\begin{array}{c}\geq \mathbf{3 0} \\
n=3196\end{array}$ & $\begin{array}{c}\text { Gesamt } \\
\mathrm{N}=27700\end{array}$ \\
\hline \multirow{2}{*}{$>90 .(\mathrm{LGA})$} & 1473 & 749 & 304 & 130 & 93 & $\overline{5527}$ & 2749 \\
\hline & $7,8 \%$ & $13,1 \%$ & $15,2 \%$ & $16,8 \%$ & $22,2 \%$ & $16,5 \%$ & $9,9 \%$ \\
\hline \multirow{2}{*}{ 10.- 90.} & 15457 & 4515 & 1548 & 575 & 285 & 2408 & 22380 \\
\hline & $82,3 \%$ & $78,8 \%$ & $77,2 \%$ & $74,4 \%$ & $68,2 \%$ & $75,3 \%$ & $80,8 \%$ \\
\hline \multirow{2}{*}{$<10 .(\mathrm{SGA})$} & 1841 & 469 & 153 & 68 & 40 & 261 & 2571 \\
\hline & $9,8 \%$ & $8,2 \%$ & $7,5 \%$ & $8,8 \%$ & $9,6 \%$ & $8,2 \%$ & $9,3 \%$ \\
\hline
\end{tabular}

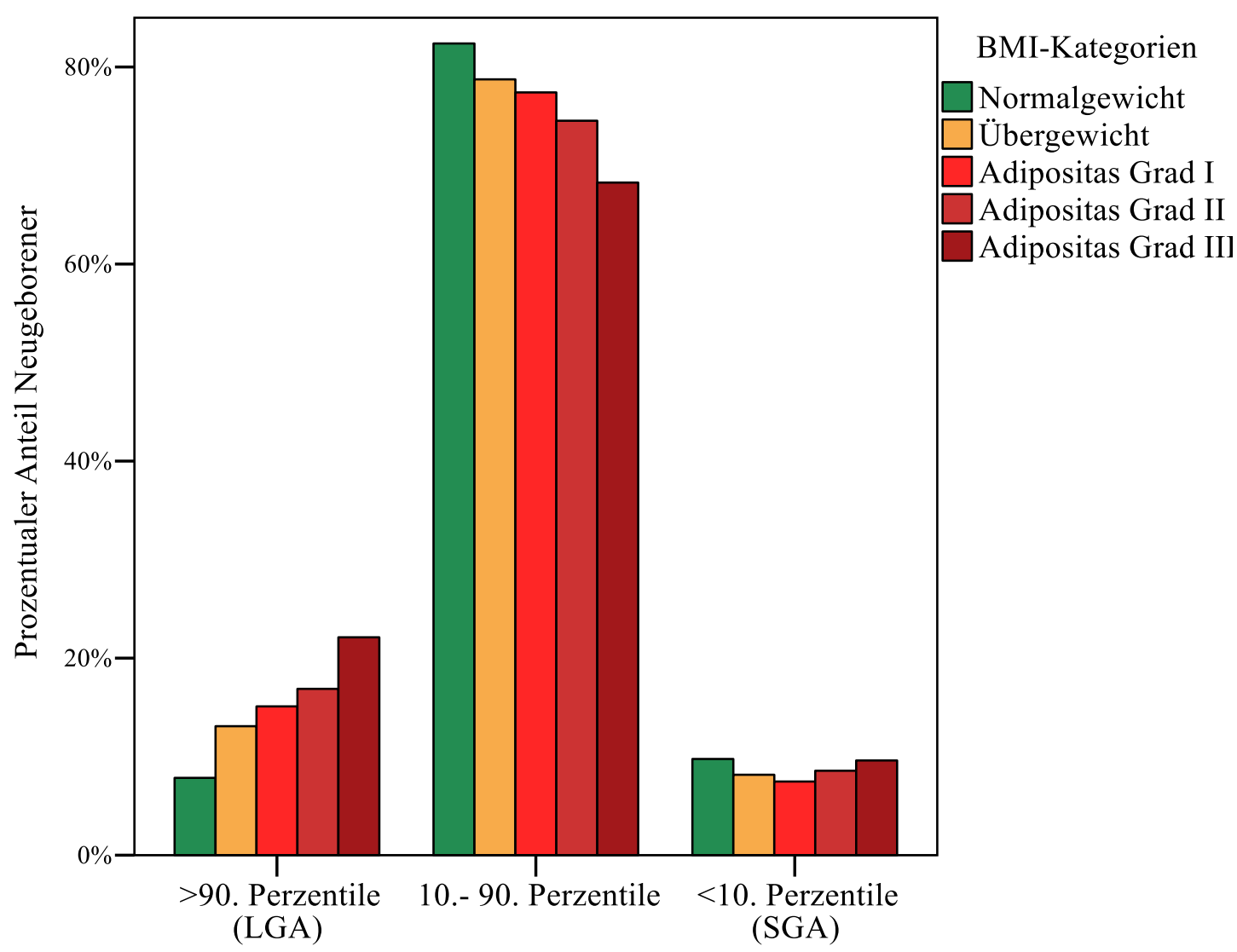

Kindliches Geburtsgewicht

Abb. 18 Relative Häufigkeitsverteilung der klassifizierten kindlichen Geburtsgewichtsperzentile in Bezug auf maternale BMI-Kategorien $(N=27700)$ 
Um den unabhängigen Effekt eines hohen BMI von mütterlichem Diabetes mellitus auf die Häufigkeit von LGA-Kindern zu prüfen, wurde die Subgruppe stoffwechselgesunder Frauen $(\mathrm{N}=25282)$ herangezogen (Tab. 58).

Die Rate der insgesamt als LGA klassifizierten Neugeborenen fiel dabei von 9,9\% im Gesamtkollektiv (2749 Fälle) auf 9,5\% (2397 Fälle) unter stoffwechselgesunden Patientinnen ab. Eine signifikante Assoziation mit dem mütterlichen BMI war weiterhin zu beobachten, wobei der Anteil der Frauen, die ein als LGA eingestuftes Kind zur Welt brachten, unter übergewichtigen mit 12,4\% und adipösen Müttern mit 15,8\% signifikant höher war im Vergleich zum Referenzkollektiv mit 7,7\% (jeweils $\mathrm{p}<0,001$; Chi-Quadrat-Test).

Somit bestätigte sich ein unabhängiger Einfluss der Adipositas von einer diabetischen Stoffwechsellage der Mutter auf das Auftreten von als LGA klassifizierten Kindern.

Tab. 58 Absolute und relative Häufigkeiten eines LGA-Kindes in Bezug auf maternale BMI-Kategorien in der Subgruppe stoffwechselgesunder Frauen

BMI-Kategorien $\left[\mathrm{kg} / \mathrm{m}^{2}\right]$

\begin{tabular}{lccccccc} 
Gewichts- & $\mathbf{1 8 , 5 - 2 4 , 9}$ & $\mathbf{2 5 , 0 - 2 9 , 9}$ & $\mathbf{3 0 , 0 - 3 4 , 9}$ & $\mathbf{3 5 , 0 - 3 9 , 9}$ & $\mathbf{2 4 0}$ & $\mathbf{2} \mathbf{3 0}$ & Gesamt \\
perzentile & $\mathrm{n}=17651$ & $\mathrm{n}=5117$ & $\mathrm{n}=1664$ & $\mathrm{n}=571$ & $\mathrm{n}=279$ & $\mathrm{n}=2514$ & $\mathrm{~N}=25282$ \\
\hline \hline$>90$. & 1367 & 632 & 245 & 95 & 58 & 398 & 2397 \\
(LGA) & $7,7 \%$ & $12,4 \%$ & $14,7 \%$ & $16,6 \%$ & $20,8 \%$ & $15,8 \%$ & $9,5 \%$ \\
\hline
\end{tabular}

\subsubsection{Apgar-Werte nach einer, fünf und zehn Minuten postnatal}

Die Apgar-Werte der Kinder adipöser Frauen lagen im Mittel nach der ersten Lebensminute mit 8,44 (SD 1,50), der fünften mit 9,49 (SD 1,10) und der zehnten Lebensminute mit 9,75 (SD 0,85) bei signifikant niedrigeren Werten, verglichen mit denen der Kinder normalgewichtiger Frauen mit Mittelwerten von 8,66 (SD 1,25) nach der ersten, 9,65 (SD 0,87) nach der fünften und 9,83 (SD 0,67) nach der zehnten Lebensminute (jeweils p<0,001; ANOVA bzw. Post-hocTests).

Zudem konnte ein Abfall des mittleren Apgar-Werts der Neugeborenen mit steigender BMIKategorie der Mutter zu allen genannten Zeitpunkten beobachtet werden (Tab. 59). 
Tab. 59 Mittelwerte und SD des kindlichen Apgar-Werts nach einer, fünf und zehn Lebensminuten in Bezug auf maternale BMI-Kategorien

BMI-Kategorien $\left[\mathrm{kg} / \mathrm{m}^{2}\right]$

\begin{tabular}{|c|c|c|c|c|c|c|c|}
\hline $\begin{array}{l}\text { Apgar-Mittel- } \\
\text { werte (SD) }\end{array}$ & $\begin{array}{r}\mathbf{1 8 , 5 - 2 4 , 9} \\
\mathrm{n}=18672 \\
\end{array}$ & $\begin{array}{c}\mathbf{2 5 , 0 - 2 9 , 9} \\
n=5694\end{array}$ & $\begin{array}{c}\mathbf{3 0 , 0 - 3 4 , 9} \\
\mathrm{n}=1991\end{array}$ & $\begin{array}{c}\mathbf{3 5 , 0 - 3 9 , 9} \\
n=768\end{array}$ & $\begin{array}{c}\geq 40 \\
n=415\end{array}$ & $\begin{array}{c}\geq \mathbf{3 0} \\
\mathrm{n}=3174\end{array}$ & $\begin{array}{c}\text { Gesamt } \\
\mathrm{N}=27540\end{array}$ \\
\hline 1 Minute & $\begin{array}{c}8,66 \\
(1,25) \\
\end{array}$ & $\begin{array}{c}8,59 \\
(1,33) \\
\end{array}$ & $\begin{array}{c}8,50 \\
(1,48) \\
\end{array}$ & $\begin{array}{c}8,43 \\
(1,39) \\
\end{array}$ & $\begin{array}{c}8,15 \\
(1,70) \\
\end{array}$ & $\begin{array}{c}8,44 \\
(1,50) \\
\end{array}$ & $\begin{array}{c}8,62 \\
(1,30) \\
\end{array}$ \\
\hline 5 Minuten & $\begin{array}{c}9,65 \\
(0,87) \\
\end{array}$ & $\begin{array}{c}9,60 \\
(0,94) \\
\end{array}$ & $\begin{array}{c}9,51 \\
(1,10) \\
\end{array}$ & $\begin{array}{c}9,48 \\
(1,03) \\
\end{array}$ & $\begin{array}{c}9,37 \\
(1,15) \\
\end{array}$ & $\begin{array}{c}9,49 \\
(1,10) \\
\end{array}$ & $\begin{array}{c}9,62 \\
(0,91) \\
\end{array}$ \\
\hline 10 Minuten & $\begin{array}{c}9,83 \\
(0,67) \\
\end{array}$ & $\begin{array}{c}9,81 \\
(0,67) \\
\end{array}$ & $\begin{array}{c}9,75 \\
(0,88) \\
\end{array}$ & $\begin{array}{c}9,76 \\
(0,74) \\
\end{array}$ & $\begin{array}{c}9,70 \\
(0,91) \\
\end{array}$ & $\begin{array}{c}9,75 \\
(0,85) \\
\end{array}$ & $\begin{array}{r}9,82 \\
(0,69) \\
\end{array}$ \\
\hline
\end{tabular}

Bei der Klassifizierung lebensfrischer Kinder und solcher mit schwer- oder mittelgradiger Depression anhand des Apgar-Werts nach der ersten, fünften und zehnten Lebensminute unterschieden sich die BMI-Gruppen Normalgewicht und Adipositas zu allen genannten Zeitpunkten signifikant voneinander.

Die lebendgeborenen Kinder adipöser Frauen wurden sowohl nach der ersten ( $p<0,001)$, fünften $(\mathrm{p}<0,001)$ und zehnten $(\mathrm{p}=0,001)$ Lebensminute mit $85,9 \%, 95,5 \%$ und $98,4 \%$ signifikant seltener als lebensfrisch eingestuft, als die Kinder normalgewichtiger Frauen mit 90,6\%, 97,2\% und $99,1 \%$. Eine schwergradige Depression des Neugeborenen wurde zu allen genannten Zeitpunkten signifikant häufiger bei adipösen Müttern festgestellt im Vergleich zu den Frauen des Referenzkollektivs (Tab. 60 bis 62; Abb. 19 bis 21).

Bei der Einschätzung nach der ersten Lebensminute war der Anteil der Kinder mit einem Apgar-Wert kleiner als acht Punkte (mittel- und schwergradige Depression) unter übergewichtigen Frauen mit 10,7\% und adipösen Frauen mit 14,1\% signifikant höher als unter normalgewichtigen Müttern mit 9,4\% ( $p=0,003$ bzw. $p<0,001$; Chi-Quadrat-Test). Die schwergradige Depression des Neugeborenen wurde bei adipösen Patientinnen mit 2,3\% (73 Fälle) signifikant häufiger ( $p<0,001)$ festgestellt als bei den Frauen des Referenzkollektivs mit 1,4\% (258 Fälle). Die absoluten und relativen Häufigkeiten der klassifizierten Apgar-Werte nach einer Minute post partum sind in Tabelle 60 sowie in Abbildung 19 in Bezug auf die mütterlichen BMIKategorien dargestellt. 
Tab. 60 Absolute und relative Häufigkeiten der klassifizierten Apgar-Werte nach der ersten Lebensminute in Bezug auf maternale BMI-Kategorien

BMI-Kategorien $\left[\mathrm{kg} / \mathrm{m}^{2}\right]$

\begin{tabular}{|c|c|c|c|c|c|c|c|}
\hline Apgar 1 Min. & $\begin{array}{c}\mathbf{1 8 , 5 - 2 4 , 9} \\
n=18672\end{array}$ & $\begin{array}{c}\mathbf{2 5 , 0 - 2 9 , 9} \\
n=5694\end{array}$ & $\begin{array}{c}\mathbf{3 0 , 0 - 3 4 , 9} \\
n=1991\end{array}$ & $\begin{array}{c}\mathbf{3 5 , 0 - 3 9 , 9} \\
n=768\end{array}$ & $\begin{array}{c}\geq 40 \\
n=415\end{array}$ & $\begin{array}{c}\geq \mathbf{3 0} \\
n=3174\end{array}$ & $\begin{array}{c}\text { Gesamt } \\
\mathrm{N}=27540\end{array}$ \\
\hline \multirow{2}{*}{ Lebensfrisch } & 16924 & 5085 & 1741 & 658 & 329 & 2728 & 24737 \\
\hline & $90,6 \%$ & $89,3 \%$ & $87,4 \%$ & $85,7 \%$ & $79,3 \%$ & $85,9 \%$ & $89,8 \%$ \\
\hline Mittelgradige & 1490 & 515 & 205 & 97 & 71 & 373 & 2378 \\
\hline Depression & $8,0 \%$ & $9,0 \%$ & $10,3 \%$ & $12,6 \%$ & $17,1 \%$ & $11,8 \%$ & $8,6 \%$ \\
\hline Schwergradige & 258 & 94 & 45 & 13 & 15 & 73 & 425 \\
\hline Depression & $1,4 \%$ & $1,7 \%$ & $2,3 \%$ & $1,7 \%$ & $3,6 \%$ & $2,3 \%$ & $1,5 \%$ \\
\hline
\end{tabular}

Nach der fünften Lebensminute war der Anteil der Neugeborenen mit einem Apgar-Wert kleiner als acht Punkte unter fettleibigen Frauen mit 4,4\% signifikant höher, als unter normalgewichtigen Patientinnen mit 2,9\% ( $p<0,001$; Chi-Quadrat-Test). Die Rate der Kinder, die als schwergradig deprimiert eingestuft wurden, war unter adipösen Frauen mit 0,6\% (20 Fälle) signifikant höher ( $\mathrm{p}=0,001)$, als unter den Frauen des Referenzkollektivs mit 0,3\% (49 Fälle). Tabelle 61 sowie Abbildung 20 zeigen die absoluten und relativen Häufigkeiten der klassifizierten Apgar-Werte nach der fünften Lebensminute entsprechend den mütterlichen BMI-Kategorien.

Tab. 61 Absolute und relative Häufigkeiten der klassifizierten Apgar-Werte nach der fünften Lebensminute in Bezug auf maternale BMI-Kategorien

BMI-Kategorien $\left[\mathrm{kg} / \mathrm{m}^{2}\right]$

\begin{tabular}{lccccccc}
\cline { 2 - 6 } Apgar 5 Min. & $\mathbf{1 8 , 5 - 2 4 , 9}$ & $\mathbf{2 5 , 0 - 2 9 , 9}$ & $\mathbf{3 0 , 0 - 3 4 , 9}$ & $\mathbf{3 5 , 0 - 3 9 , 9}$ & $\mathbf{2 4 0}$ & $\mathbf{2} 30$ & Gesamt \\
& $\mathrm{n}=18672$ & $\mathrm{n}=5694$ & $\mathrm{n}=1991$ & $\mathrm{n}=768$ & $\mathrm{n}=415$ & $\mathrm{n}=3174$ & $\mathrm{~N}=27540$ \\
\hline \hline \multirow{2}{*}{ Lebensfrisch } & 18142 & 5510 & 1898 & 743 & 391 & 3032 & 26684 \\
& $97,2 \%$ & $96,8 \%$ & $95,3 \%$ & $96,7 \%$ & $94,2 \%$ & $95,5 \%$ & $96,9 \%$ \\
\hline Mittelgradige & 481 & 165 & 80 & 21 & 21 & 122 & 768 \\
Depression & $2,6 \%$ & $2,9 \%$ & $4,0 \%$ & $2,7 \%$ & $5,1 \%$ & $3,8 \%$ & $2,8 \%$ \\
\hline Schwergradige & 49 & 19 & 13 & 4 & 3 & 20 & 88 \\
Depression & $0,3 \%$ & $0,3 \%$ & $0,7 \%$ & $0,5 \%$ & $0,7 \%$ & $0,6 \%$ & $0,3 \%$ \\
\hline
\end{tabular}

Nach der zehnten Minute postnatal war die Rate der Kinder mit einem Apgar-Wert kleiner als 8 Punkte unter übergewichtigen Frauen mit 1,3\% (72 Fälle) und adipösen Frauen mit 1,6\% (50 Fälle) signifikant höher ( $\mathrm{p}=0,020$ bzw. $\mathrm{p}=0,001$; Chi-Quadrat-Test) als in der Gruppe der normalgewichtigen Frauen mit 1,0\% (171 Fälle). Der Anteil der Kinder mit einer schwergradigen Depression war nach der zehnten Lebensminute unter fettleibigen Frauen mit 0,5\% (16 Fälle) signifikant höher $(\mathrm{p}=0,014)$ als unter den Frauen des Referenzkollektivs mit 0,3\% (47 Fälle). 
Tabelle 62 bzw. Abbildung 21 stellen die absoluten und relativen Häufigkeiten der klassifizierten Apgar-Werte nach der zehnten Minute postnatal in Bezug auf die mütterlichen BMI-Kategorien dar.

Tab. 62 Absolute und relative Häufigkeiten der klassifizierten Apgar-Werte nach der zehnten Lebensminute in Bezug auf maternale BMI-Kategorien

BMI-Kategorien $\left[\mathrm{kg} / \mathrm{m}^{2}\right]$

\begin{tabular}{|c|c|c|c|c|c|c|c|}
\hline Apgar 10 Min. & $\begin{array}{r}\mathbf{1 8 , 5 - 2 4 , 9} \\
\mathrm{n}=18672\end{array}$ & $\begin{array}{c}\mathbf{2 5 , 0 - 2 9 , 9} \\
n=5694\end{array}$ & $\begin{array}{c}\mathbf{3 0 , 0 - 3 4 , 9} \\
\mathrm{n}=1991\end{array}$ & $\begin{array}{c}\mathbf{3 5 , 0 - 3 9 , 9} \\
n=768\end{array}$ & $\begin{array}{c}\geq \mathbf{4 0} \\
\mathrm{n}=415\end{array}$ & $\begin{array}{c}\geq \mathbf{3 0} \\
n=3174\end{array}$ & $\begin{array}{c}\text { Gesamt } \\
\mathrm{N}=27540\end{array}$ \\
\hline \multirow{2}{*}{ Lebensfrisch } & 18501 & 5622 & 1957 & 759 & 408 & 3142 & 27247 \\
\hline & $99,1 \%$ & $98,7 \%$ & $98,3 \%$ & $98,8 \%$ & $98,3 \%$ & $98,4 \%$ & $98,9 \%$ \\
\hline Mittelgradige & 124 & 60 & 23 & 7 & 4 & 34 & 218 \\
\hline Depression & $0,7 \%$ & $1,1 \%$ & $1,2 \%$ & $0,9 \%$ & $1,0 \%$ & $1,1 \%$ & $0,8 \%$ \\
\hline Schwergradige & 47 & 12 & 11 & 2 & 3 & 16 & 75 \\
\hline Depression & $0,3 \%$ & $0,2 \%$ & $0,6 \%$ & $0,3 \%$ & $0,7 \%$ & $0,5 \%$ & $0,3 \%$ \\
\hline
\end{tabular}

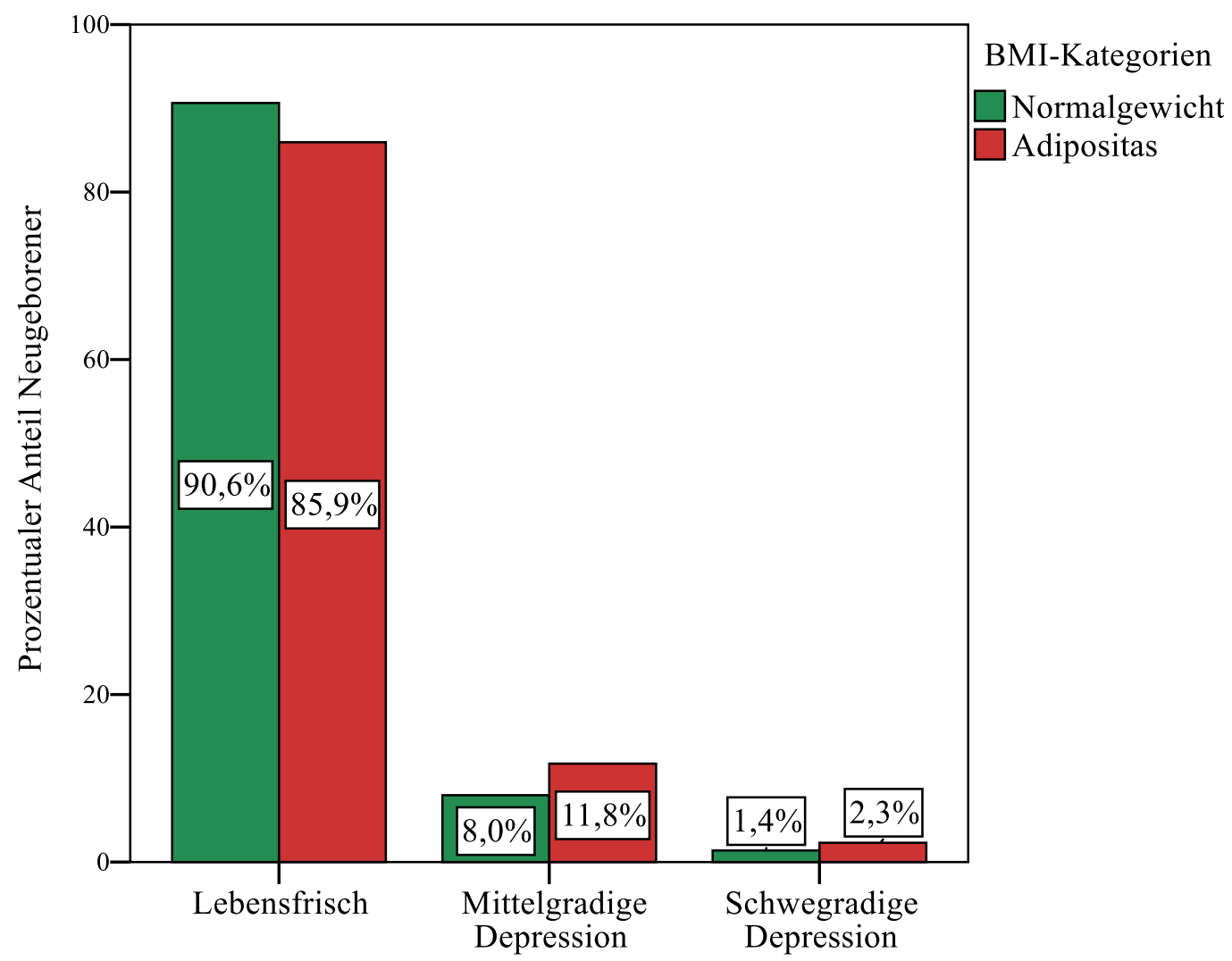

Apgar-Werte nach der ersten Lebensminute

Abb. 19 Relative Häufigkeitsverteilung der klassifizierten Apgar-Werte nach der ersten Lebensminute in Bezug auf maternale BMI-Kategorien $(N=27540)$ 


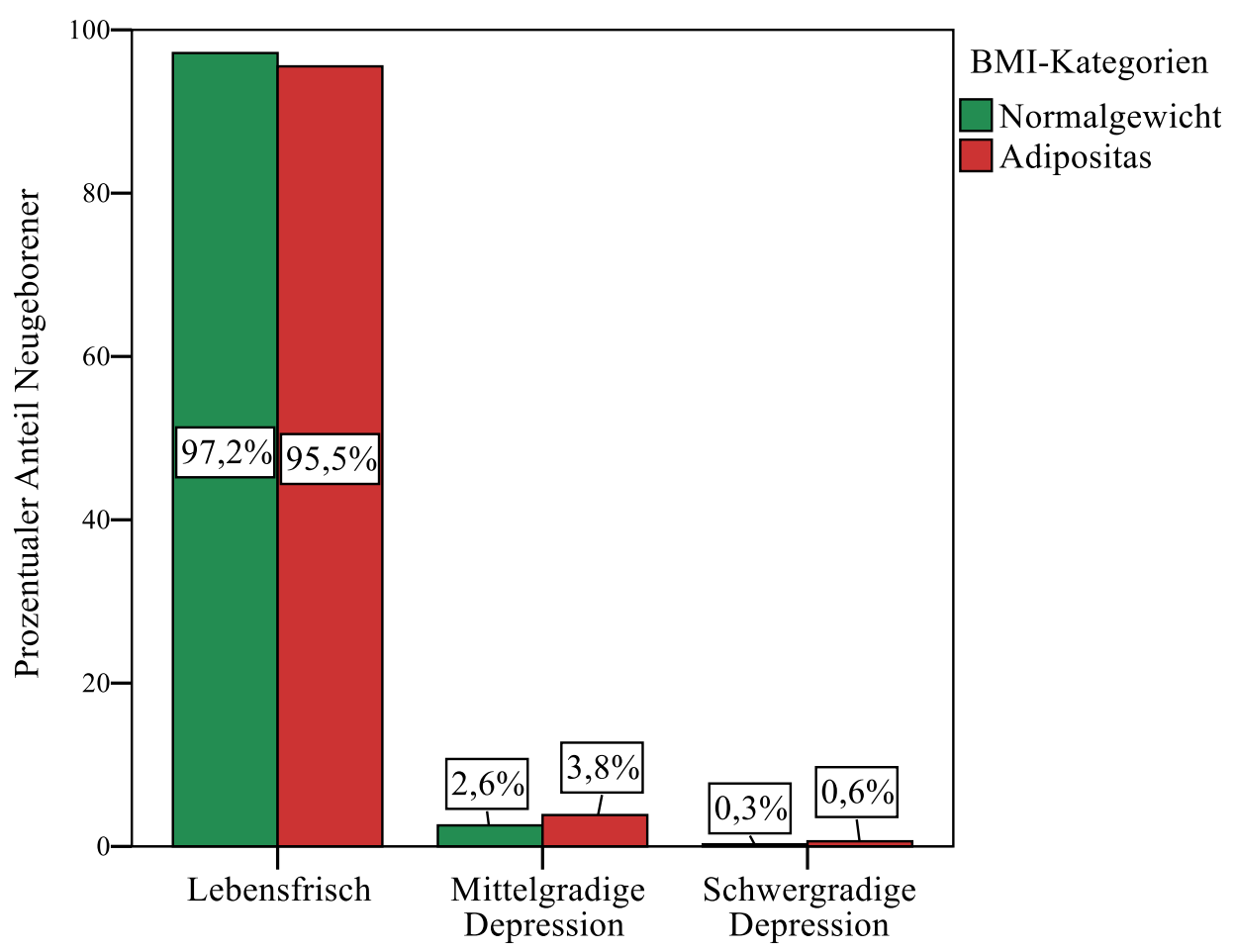

Apgar-Werte nach der fünften Lebensminute

Abb. 20 Relative Häufigkeitsverteilung der klassifizierten Apgar-Werte nach der fünften Lebensminute in Bezug auf maternale BMI-Kategorien $(N=27540)$

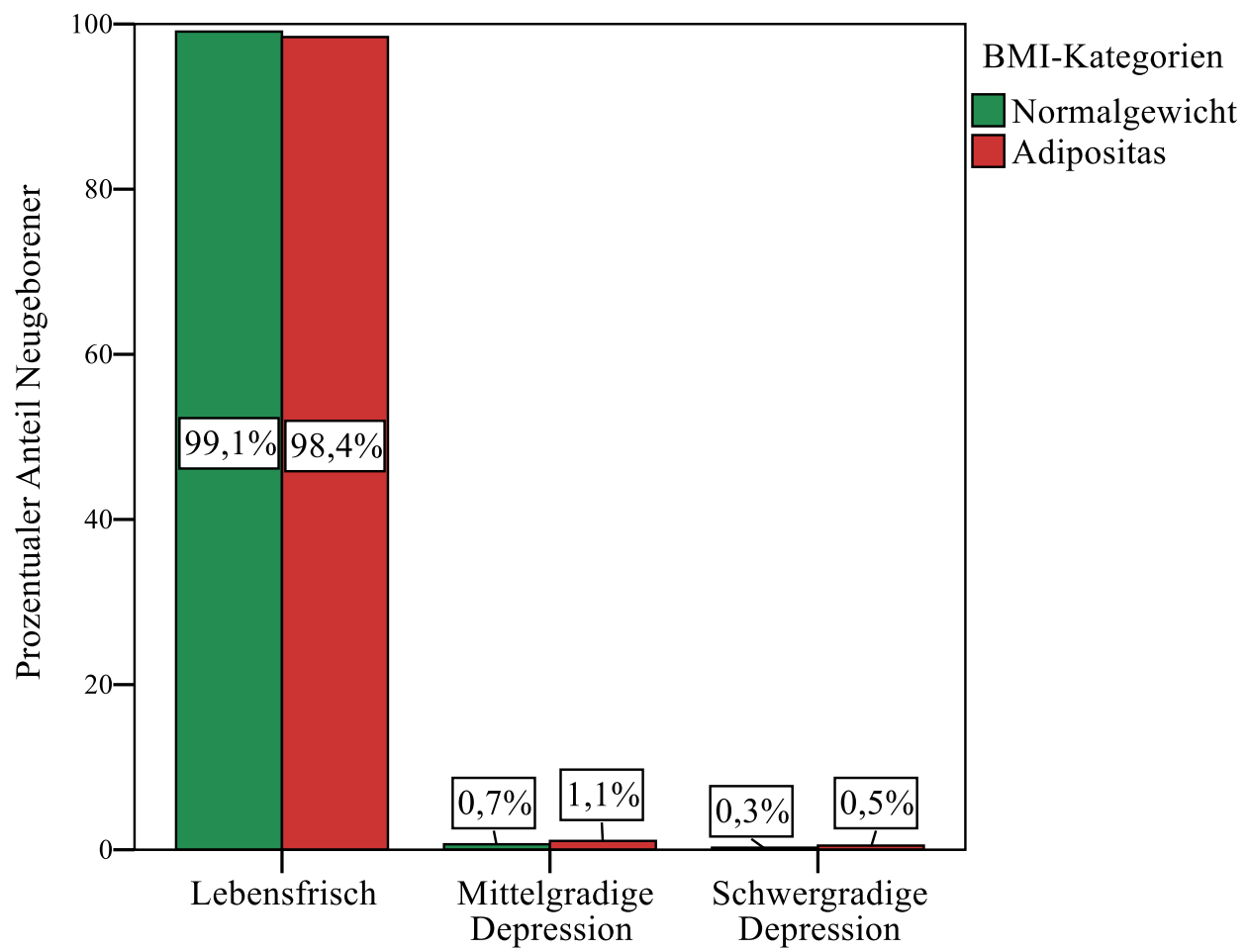

Apgar-Werte nach der zehnten Lebensminute

Abb. 21 Relative Häufigkeitsverteilung der klassifizierten Apgar-Werte nach der zehnten Lebensminute in Bezug auf maternale BMI-Kategorien $(N=27540)$ 


\subsubsection{PH- und BE-Wert im Nabelarterienblut}

Beim Vergleich der pH-Mittelwerte im Nabelarterienblut der Neugeborenen in Abhängigkeit des mütterlichen BMI fiel die Tendenz sinkender kindlicher $\mathrm{pH}-$ Werte mit steigender BMIKategorie der Mutter auf (Tab. 63).

Die einfaktorielle Varianzanalyse zeigte einen signifikanten Unterschied zwischen den BMIGruppen $(\mathrm{p}=0,018)$. Neugeborene adipöser Frauen unterschieden sich in den paarweisen Mehrfachvergleichen mit einem mittleren pH-Wert von 7,267 (SD 0,090) im Nabelschnurblut signifikant ( $\mathrm{p}=0,005)$ von denen normalgewichtiger Frauen mit 7,272 (SD 0,086).

Tab. 63 Mittelwert und SD des kindlichen $\mathrm{pH}$-Werts im Nabelarterienblut in Bezug auf maternale BMI-Kategorien BMI-Kategorien $\left[\mathrm{kg} / \mathrm{m}^{2}\right]$

\begin{tabular}{lccccccc}
\cline { 2 - 6 } pH-Wert & $\begin{array}{c}\mathbf{1 8 , 5 - 2 4 , 9} \\
\mathrm{n}=18628\end{array}$ & $\begin{array}{c}\mathbf{2 5 , 0 - 2 9 , 9} \\
\mathrm{n}=5701\end{array}$ & $\begin{array}{c}\mathbf{3 0 , 0 - 3 4 , 9} \\
\mathrm{n}=1984\end{array}$ & $\begin{array}{c}\mathbf{3 5 , 0 - 3 9 , 9} \\
\mathrm{n}=766\end{array}$ & $\begin{array}{c}\mathbf{2 4 0} \\
\mathrm{n}=413\end{array}$ & $\begin{array}{c}\mathbf{2 3 0} \\
\mathrm{n}=3163\end{array}$ & $\begin{array}{c}\text { Gesamt } \\
\mathrm{N}=27492\end{array}$ \\
\hline \hline Mittelwert & 7,272 & 7,271 & 7,268 & 7,263 & 7,260 & 7,267 & 7,270 \\
\hline SD & 0,086 & 0,087 & 0,090 & 0,087 & 0,093 & 0,090 & 0,087 \\
\hline
\end{tabular}

Tabelle 64 und Abbildung 22 zeigen die absoluten und relativen Häufigkeiten des in verschiedene Azidoseschweregrade klassifizierten kindlichen pH-Werts in Abhängigkeit der mütterlichen BMI-Kategorie.

Im Nabelarterienblut der Kinder übergewichtiger und adipöser Frauen wurde mit 2,7\% (155 Fälle) bzw. 3,1\% (97 Fälle) signifikant häufiger ( $\mathrm{p}=0,012$ bzw. $\mathrm{p}=0,001)$ ein $\mathrm{pH}-$ Wert kleiner als 7,1 (fortgeschrittene oder schwere Azidose) festgestellt als bei den Kindern normalgewichtiger Frauen mit 2,1\% (401 Fälle).

Eine schwere Azidose mit einem pH-Wert kleiner als 7,0 wurde im Untersuchungszeitraum insgesamt bei 0,3\% der Neugeborenen diagnostiziert (75 Fälle), wobei der Anteil unter den Kindern fettleibiger Frauen mit 0,5\% (16 Fälle) signifikant größer war ( $\mathrm{p}=0,006)$, als unter den Neugeborenen des Referenzkollektivs mit 0,2\% (43 Fälle).

Der prozentuale Anteil der Mütter, deren Neugeborene dagegen mit einem pH-Wert von mindestens 7,2 keine oder lediglich eine leichte Azidose aufwiesen, war unter normalgewichtigen Frauen mit 80,9\% signifikant höher $(\mathrm{p}=0,038)$ als unter den Frauen mit einem BMI $\geq 30 \mathrm{~kg} / \mathrm{m}^{2}$ mit $79,4 \%$.

Die Prüfung der statistischen Signifikanz erfolgte in allen Fällen über den Chi-Quadrat-Test. 
Tab. 64 Absolute und relative Häufigkeiten des klassifizierten kindlichen pH-Werts im Nabelarterienblut in Bezug auf maternale BMI-Kategorien

BMI-Kategorien $\left[\mathrm{kg} / \mathrm{m}^{2}\right]$

\begin{tabular}{lccccccc}
\cline { 2 - 6 } Azidose- & $\mathbf{1 8 , 5 - 2 4 , 9}$ & $\mathbf{2 5 , 0 - 2 9 , 9}$ & $\mathbf{3 0 , 0 - 3 4 , 9}$ & $\mathbf{3 5 , 0 - 3 9 , 9}$ & $\mathbf{2 4 0}$ & $\mathbf{2 3 0}$ & Gesamt \\
schweregrad & $\mathrm{n}=18628$ & $\mathrm{n}=5701$ & $\mathrm{n}=1984$ & $\mathrm{n}=766$ & $\mathrm{n}=413$ & $\mathrm{n}=3163$ & $\mathrm{~N}=27492$ \\
\hline \hline Keine & 7771 & 2378 & 811 & 311 & 158 & 1280 & 11429 \\
$\geq 7,30$ & $41,7 \%$ & $41,7 \%$ & $40,9 \%$ & $40,6 \%$ & $38,3 \%$ & $40,5 \%$ & $41,6 \%$ \\
\hline Leicht & 7305 & 2221 & 755 & 311 & 164 & 1230 & 10756 \\
$7,20-7,29$ & $39,2 \%$ & $39,0 \%$ & $38,1 \%$ & $40,6 \%$ & $39,7 \%$ & $38,9 \%$ & $39,1 \%$ \\
\hline Mittelgradig & 3151 & 947 & 356 & 128 & 72 & 556 & 4654 \\
$7,10-7,19$ & $16,9 \%$ & $16,6 \%$ & $17,9 \%$ & $16,7 \%$ & $17,4 \%$ & $17,6 \%$ & $16,9 \%$ \\
\hline Fortgeschritten & 358 & 139 & 51 & 14 & 16 & 81 & 578 \\
$7,00-7,09$ & $1,9 \%$ & $2,4 \%$ & $2,6 \%$ & $1,8 \%$ & $3,9 \%$ & $2,6 \%$ & $2,1 \%$ \\
\hline Schwer & 43 & 16 & 11 & 2 & 3 & 16 & 75 \\
$<7,00$ & $0,2 \%$ & $0,3 \%$ & $0,6 \%$ & $0,3 \%$ & $0,7 \%$ & $0,5 \%$ & $0,3 \%$ \\
\hline
\end{tabular}

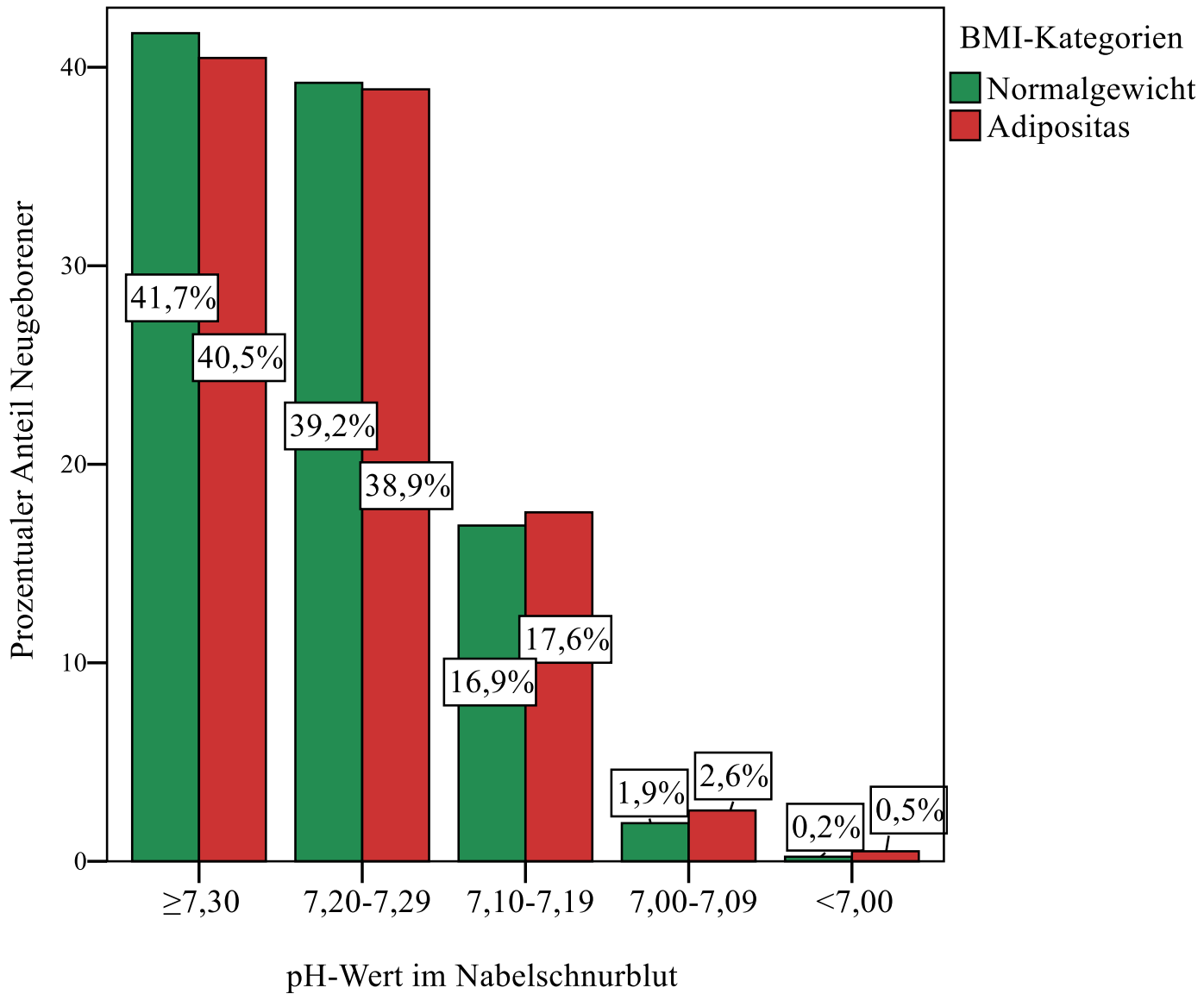

Abb. 22 Relative Häufigkeitsverteilung des klassifizierten kindlichen pH-Werts im Nabelarterienblut in Bezug auf mütterliche BMI-Kategorien (N=27492) 
Hinsichtlich des kindlichen BE-Werts im Nabelarterienblut zeigte die einfaktorielle Varianzanalyse einen signifikanten Unterschied zwischen den BMI-Gruppen ( $\mathrm{p}=0,048)$. Die Neugeborenen adipöser Frauen unterschieden sich mit einem BE-Mittelwert von -3,99 mmol/l (SD $3,51)$ in den Post-hoc Tests signifikant $(\mathrm{p}=0,014)$ von denen normalgewichtiger Frauen mit $-4,15 \mathrm{mmol} / \mathrm{l}(\mathrm{SD} \mathrm{3,36)}$.

Beim Vergleich der mittleren BE-Werte im Nabelschnurblut der Neugeborenen in Bezug auf die mütterliche BMI-Gruppe zeichnete sich zudem die Tendenz zunehmender kindlicher BEWerte mit steigender BMI-Kategorie der Mutter ab (Tab. 65).

Tab. 65 Mittelwert und SD des kindlichen BE-Werts im Nabelarterienblut in Bezug aufmaternale BMI-Kategorien BMI-Kategorien $\left[\mathrm{kg} / \mathrm{m}^{2}\right]$

\begin{tabular}{lcccccccc} 
& \multicolumn{7}{c}{ BMI-Kategorien $\left[\mathrm{kg} / \mathrm{m}^{2}\right]$} \\
\cline { 2 - 7 } BE-Wert & $\begin{array}{c}\mathbf{1 8 , 5 - 2 4 , 9} \\
\mathrm{n}=18552\end{array}$ & $\begin{array}{c}\mathbf{2 5 , 0 - 2 9 , 9} \\
\mathrm{n}=5685\end{array}$ & $\begin{array}{c}\mathbf{3 0 , 0 - 3 4 , 9} \\
\mathrm{n}=1977\end{array}$ & $\begin{array}{c}\mathbf{3 5 , 0 - 3 9 , 9} \\
\mathrm{n}=766\end{array}$ & $\begin{array}{c}\mathbf{2 4 0} \\
\mathrm{n}=410\end{array}$ & $\begin{array}{c}\mathbf{2 3 0} \\
\mathrm{n}=3153\end{array}$ & $\begin{array}{c}\text { Gesamt } \\
\text { N=27391 }\end{array}$ \\
\hline \hline Mittelwert & $-4,15$ & $-4,13$ & $-4,06$ & $-3,87$ & $-3,86$ & $-3,99$ & $-4,13$ \\
\hline SD & 3,36 & 3,44 & 3,50 & 3,44 & 3,66 & 3,51 & 3,39 \\
\hline
\end{tabular}

\subsubsection{Fehlbildungen}

Ein signifikanter Zusammenhang zwischen der Häufigkeit einer kindlichen Fehlbildung und dem BMI der Mutter konnte in der vorliegenden Studie nicht festgestellt werden, wobei insgesamt 537 Neugeborene (1,9\%) von einer kongenitalen Anomalie betroffen waren (Tab. 66).

Unter den Kindern normalgewichtiger Frauen wiesen 1,9\% (356 Fälle) eine Fehlbildung auf, unter übergewichtigen Frauen war der Anteil vergleichsweise mit 2,2\% (124 Fälle) etwas höher ( $\mathrm{p}=0,198)$ und unter adipösen Frauen mit 1,8\% (57 Fälle) etwas niedriger ( $\mathrm{p}=0,663$; Chi-Quadrat-Test).

Tab. 66 Absolute und relative Häufigkeiten einer kindlichen Fehlbildung in Bezug auf maternale BMI-Kategorien BMI-Kategorien $\left[\mathrm{kg} / \mathrm{m}^{2}\right]$

\begin{tabular}{|c|c|c|c|c|c|c|c|}
\hline Fehlbildung & $\begin{array}{c}\mathbf{1 8 , 5 - 2 4 , 9} \\
\mathrm{n}=18806\end{array}$ & $\begin{array}{c}\mathbf{2 5 , 0 - 2 9 , 9} \\
n=5736\end{array}$ & $\begin{array}{c}\mathbf{3 0 , 0 - 3 4 , 9} \\
n=2009\end{array}$ & $\begin{array}{c}\mathbf{3 5 , 0 - 3 9 , 9} \\
\mathrm{n}=773\end{array}$ & $\begin{array}{c}\geq \mathbf{4 0} \\
\mathrm{n}=420\end{array}$ & $\begin{array}{c}\geq \mathbf{3 0} \\
n=3202\end{array}$ & $\begin{array}{c}\text { Gesamt } \\
\mathrm{N}=27744\end{array}$ \\
\hline \multirow{2}{*}{$\mathrm{Ja}$} & 356 & 124 & 38 & 14 & 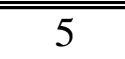 & $\overline{57}$ & "537 \\
\hline & $1,9 \%$ & $2,2 \%$ & $1,9 \%$ & $1,8 \%$ & $1,2 \%$ & $1,8 \%$ & $1,9 \%$ \\
\hline \multirow{2}{*}{ Nein } & 18450 & 5612 & 1971 & 579 & 415 & 3154 & 27207 \\
\hline & $98,1 \%$ & $97,8 \%$ & $98,1 \%$ & $98,2 \%$ & $98,8 \%$ & $98,2 \%$ & $98,1 \%$ \\
\hline
\end{tabular}


Insgesamt lag bei 341 der fehlgebildeten Kinder $(63,6 \%)$ bereits pränatal die Diagnose vor (Tab. 67). Die Rate der Fehlbildungen, die pränatal festgestellt werden konnte, war unter übergewichtigen Schwangeren mit 68,5\% am höchsten, gefolgt von normalgewichtigen Patientinnen mit 63,5\% und schließlich adipösen Frauen, unter denen der Anteil einer pränatalen Detektion der kongenitalen Anomalie mit 53,6\% am niedrigsten war. Die Gegenüberstellung der BMI-Gruppen Übergewicht und Adipositas mit der Referenzgruppe zeigte im Chi-QuadratTest mit $\mathrm{p}=0,155$ bzw. $\mathrm{p}=0,309$ jeweils kein statistisch signifikantes Ergebnis.

Tab. 67 Absolute und relative Häufigkeiten einer pränatalen Diagnose unter allen fehlgebildeten Kindern in Bezug auf maternale BMI-Kategorien

BMI-Kategorien $\left[\mathrm{kg} / \mathrm{m}^{2}\right]$

\begin{tabular}{lccccccc} 
Pränatale & $\mathbf{1 8 , 5 - 2 4 , 9}$ & $\mathbf{2 5 , 0 - 2 9 , 9}$ & $\mathbf{3 0 , 0 - 3 4 , 9}$ & $\mathbf{3 5 , 0 - 3 9 , 9}$ & $\mathbf{2} \mathbf{4 0}$ & $\mathbf{2} \mathbf{3 0}$ & Gesamt \\
Diagnose & $\mathrm{n}=356$ & $\mathrm{n}=124$ & $\mathrm{n}=37$ & $\mathrm{n}=14$ & $\mathrm{n}=5$ & $\mathrm{n}=56$ & $\mathrm{~N}=536$ \\
\hline \hline \multirow{2}{*}{ Ja } & 226 & 85 & 17 & 9 & 4 & 30 & 341 \\
& $63,5 \%$ & $68,5 \%$ & $45,9 \%$ & $64,3 \%$ & $80,0 \%$ & $53,6 \%$ & $63,6 \%$ \\
\hline \multirow{2}{*}{ Nein } & 130 & 39 & 20 & 5 & 1 & 26 & 195 \\
& $36,5 \%$ & $31,5 \%$ & $54,1 \%$ & $35,7 \%$ & $20,0 \%$ & $46,4 \%$ & $36,4 \%$ \\
\hline
\end{tabular}

In Tabelle 68 sind die absoluten und relativen Häufigkeiten der verschiedenen Arten einer kindlichen Fehlbildung, die im Untersuchungszeitraum auftraten, in Bezug auf die mütterlichen BMI-Kategorien dargestellt. Dabei sind Mehrfachnennungen möglich, da bei einem Kind gegebenenfalls mehrere Fehlbildungen gleichzeitig vorlagen. Die angegebenen, durch den ChiQuadrat-Test bzw. den exakten Test nach Fisher bestimmten, p-Werte beziehen sich auf den Vergleich der maternalen BMI-Gruppen Normalgewicht und Adipositas.

Fehlbildungen des kindlichen Muskel-Skelett-Systems traten mit insgesamt 22,9\% (123 Fälle) am häufigsten auf, gefolgt von kardiovaskulären Fehlbildungen mit 16,8\% (90 Fälle), Anomalien des Harn- und Verdauungssystems mit 15,1\% (81 Fälle) bzw. 11,0\% (59 Fälle) sowie Fehlbildungen des Nervensystems mit 10,4\% (56 Fälle). Chromosomenanomalien und Abnormitäten der Genitalorgane machten einen Anteil von 9,1\% (49 Fälle) bzw. 9,0\% (48 Fälle) der Diagnosen aus. Lippen- und/oder Gaumenspalten lagen in 7,4\% der Fälle vor (40 Fälle) und eine kindliche Anomalie hinsichtlich Auge, Ohr, Gesicht und Hals in 3,9\% (21 Fälle). 
Eine statistisch signifikante Assoziation mit dem mütterlichen BMI war hinsichtlich keiner der genannten Fehlbildungsarten zu beobachten. Auch bei der Betrachtung der Anomalieformen Gastroschisis ( $n=10)$, Fehlbildungen der Herzsepten ( $n=49)$, Spina bifida $(n=20)$, Hydrozephalus ( $\mathrm{n}=15)$ und Hypospadie $(\mathrm{n}=25)$ für sich war kein statistisch signifikanter Zusammenhang mit mütterlicher Adipositas zu finden.

Tab. 68 Absolute und relative Häufigkeiten der verschiedenen Arten einer kindlichen Fehlbildung in Bezug auf maternale BMI-Kategorien

BMI-Kategorien [ $\left.\mathrm{kg} / \mathrm{m}^{2}\right]$

\begin{tabular}{|c|c|c|c|c|c|c|c|c|}
\hline $\begin{array}{l}\text { Art der } \\
\text { Fehlbildung }\end{array}$ & $\begin{array}{c}\mathbf{1 8 , 5 - 2 4 , 9} \\
n=356\end{array}$ & $\begin{array}{c}\mathbf{2 5 , 0 - 2 9 , 9} \\
n=124\end{array}$ & $\begin{array}{c}\mathbf{3 0 , 0 - 3 4 , 9} \\
n=38\end{array}$ & $\begin{array}{c}35,0-39,9 \\
n=14\end{array}$ & $\begin{array}{l}\geq 40 \\
n=5\end{array}$ & $\begin{array}{l}\geq \mathbf{3 0} \\
\mathrm{n}=57\end{array}$ & $\begin{array}{l}\text { Gesamt } \\
\mathrm{N}=537\end{array}$ & P-Wert \\
\hline $\begin{array}{l}\text { Muskel-Skelett- } \\
\text { System gesamt }\end{array}$ & $\begin{array}{c}88 \\
24,7 \%\end{array}$ & $\begin{array}{c}21 \\
16,9 \%\end{array}$ & $\begin{array}{c}10 \\
26,3 \%\end{array}$ & $\begin{array}{c}3 \\
21,4 \%\end{array}$ & $\begin{array}{c}1 \\
20,0 \%\end{array}$ & $\begin{array}{c}14 \\
24,6 \%\end{array}$ & $\begin{array}{c}123 \\
22,9 \%\end{array}$ & 0,980 \\
\hline Gastroschisis & $\begin{array}{c}9 \\
2,5 \% \\
\end{array}$ & $\begin{array}{c}1 \\
0,8 \% \\
\end{array}$ & $\begin{array}{c}0 \\
0,0 \% \\
\end{array}$ & $\begin{array}{c}0 \\
0,0 \% \\
\end{array}$ & $\begin{array}{c}0 \\
0,0 \% \\
\end{array}$ & $\begin{array}{c}0 \\
0,0 \% \\
\end{array}$ & $\begin{array}{c}10 \\
1,9 \% \\
\end{array}$ & 0,618 \\
\hline $\begin{array}{l}\text { Kardiovaskulär } \\
\text { gesamt }\end{array}$ & $\begin{array}{c}66 \\
18,5 \%\end{array}$ & $\begin{array}{c}19 \\
15,3 \%\end{array}$ & $\begin{array}{c}2 \\
5,3 \%\end{array}$ & $\begin{array}{c}3 \\
21,4 \%\end{array}$ & $\begin{array}{c}0 \\
0,0 \%\end{array}$ & $\begin{array}{c}5 \\
8,8 \%\end{array}$ & $\begin{array}{c}90 \\
16,8 \%\end{array}$ & 0,070 \\
\hline Herzsepten & $\begin{array}{c}34 \\
9,6 \% \\
\end{array}$ & $\begin{array}{c}12 \\
9,7 \% \\
\end{array}$ & $\begin{array}{c}0 \\
0,0 \% \\
\end{array}$ & $\begin{array}{c}3 \\
21,4 \% \\
\end{array}$ & $\begin{array}{c}0 \\
0,0 \% \\
\end{array}$ & $\begin{array}{c}3 \\
5,3 \% \\
\end{array}$ & $\begin{array}{c}49 \\
9,1 \% \\
\end{array}$ & 0,293 \\
\hline $\begin{array}{l}\text { Harnsystem } \\
\text { gesamt }\end{array}$ & $\begin{array}{c}52 \\
14,6 \% \\
\end{array}$ & $\begin{array}{c}23 \\
18,5 \% \\
\end{array}$ & $\begin{array}{c}5 \\
13,2 \% \\
\end{array}$ & $\begin{array}{c}1 \\
7,1 \% \\
\end{array}$ & $\begin{array}{c}0 \\
0,0 \% \\
\end{array}$ & $\begin{array}{c}6 \\
10,5 \% \\
\end{array}$ & $\begin{array}{c}81 \\
15,1 \% \\
\end{array}$ & 0,410 \\
\hline $\begin{array}{l}\text { Verdauungs- } \\
\text { system gesamt } \\
\end{array}$ & $\begin{array}{c}33 \\
9,2 \% \\
\end{array}$ & $\begin{array}{c}19 \\
15,3 \% \\
\end{array}$ & $\begin{array}{c}3 \\
7,9 \% \\
\end{array}$ & $\begin{array}{c}3 \\
21,4 \% \\
\end{array}$ & $\begin{array}{c}1 \\
20,0 \% \\
\end{array}$ & $\begin{array}{c}7 \\
12,3 \% \\
\end{array}$ & $\begin{array}{c}59 \\
11,0 \% \\
\end{array}$ & 0,475 \\
\hline $\begin{array}{l}\text { Nervensystem } \\
\text { gesamt }\end{array}$ & $\begin{array}{c}34 \\
9,6 \%\end{array}$ & $\begin{array}{c}15 \\
12,1 \%\end{array}$ & $\begin{array}{c}5 \\
13,2 \% \\
\end{array}$ & $\begin{array}{c}1 \\
7,1 \%\end{array}$ & $\begin{array}{c}1 \\
20,0 \%\end{array}$ & $\begin{array}{c}7 \\
12,3 \%\end{array}$ & $\begin{array}{c}56 \\
10,4 \% \\
\end{array}$ & 0,522 \\
\hline Spina bifida & $\begin{array}{c}12 \\
3,4 \% \\
\end{array}$ & $\begin{array}{c}5 \\
4,0 \% \\
\end{array}$ & $\begin{array}{c}2 \\
5,3 \% \\
\end{array}$ & $\begin{array}{c}1 \\
7,1 \% \\
\end{array}$ & $\begin{array}{c}0 \\
0,0 \% \\
\end{array}$ & $\begin{array}{c}3 \\
5,3 \% \\
\end{array}$ & $\begin{array}{c}20 \\
3,7 \% \\
\end{array}$ & 0,446 \\
\hline Hydrozephalus & $\begin{array}{c}10 \\
2,8 \% \\
\end{array}$ & $\begin{array}{c}4 \\
3,2 \% \\
\end{array}$ & $\begin{array}{c}0 \\
0,0 \% \\
\end{array}$ & $\begin{array}{c}0 \\
0,0 \% \\
\end{array}$ & $\begin{array}{c}1 \\
20,0 \% \\
\end{array}$ & $\begin{array}{c}1 \\
1,8 \% \\
\end{array}$ & $\begin{array}{c}15 \\
2,8 \% \\
\end{array}$ & 1,000 \\
\hline $\begin{array}{l}\text { Chromosomen- } \\
\text { anomalien }\end{array}$ & $\begin{array}{c}38 \\
10,7 \% \\
\end{array}$ & $\begin{array}{c}7 \\
5,6 \% \\
\end{array}$ & $\begin{array}{c}3 \\
7,9 \% \\
\end{array}$ & $\begin{array}{c}0 \\
0,0 \% \\
\end{array}$ & $\begin{array}{c}1 \\
20,0 \% \\
\end{array}$ & $\begin{array}{c}4 \\
7,0 \% \\
\end{array}$ & $\begin{array}{c}49 \\
9,1 \% \\
\end{array}$ & 0,396 \\
\hline $\begin{array}{l}\text { Genitalorgane } \\
\text { gesamt }\end{array}$ & $\begin{array}{c}32 \\
9,0 \%\end{array}$ & $\begin{array}{c}10 \\
8,1 \%\end{array}$ & $\begin{array}{c}4 \\
10,5 \%\end{array}$ & $\begin{array}{c}1 \\
7,1 \%\end{array}$ & $\begin{array}{c}1 \\
20,0 \%\end{array}$ & $\begin{array}{c}6 \\
10,5 \%\end{array}$ & $\begin{array}{c}48 \\
9,0 \%\end{array}$ & 0,709 \\
\hline Hypospadie & $\begin{array}{c}18 \\
5,1 \% \\
\end{array}$ & $\begin{array}{c}3 \\
2,4 \% \\
\end{array}$ & $\begin{array}{c}3 \\
7,9 \% \\
\end{array}$ & $\begin{array}{c}1 \\
7,1 \% \\
\end{array}$ & $\begin{array}{c}0 \\
0,0 \% \\
\end{array}$ & $\begin{array}{c}4 \\
7,0 \% \\
\end{array}$ & $\begin{array}{c}25 \\
4,7 \% \\
\end{array}$ & 0,525 \\
\hline $\begin{array}{l}\text { Lippen- und/oder } \\
\text { Gaumenspalte }\end{array}$ & $\begin{array}{c}24 \\
6,7 \% \\
\end{array}$ & $\begin{array}{c}8 \\
6,5 \% \\
\end{array}$ & $\begin{array}{c}4 \\
10,5 \% \\
\end{array}$ & $\begin{array}{c}3 \\
21,4 \% \\
\end{array}$ & $\begin{array}{c}1 \\
20,0 \% \\
\end{array}$ & $\begin{array}{c}8 \\
14,0 \% \\
\end{array}$ & $\begin{array}{c}40 \\
7,4 \% \\
\end{array}$ & 0,064 \\
\hline $\begin{array}{l}\text { Auge, Ohr, } \\
\text { Gesicht, Hals } \\
\end{array}$ & $\begin{array}{c}13 \\
3,7 \% \\
\end{array}$ & $\begin{array}{c}6 \\
4,8 \% \\
\end{array}$ & $\begin{array}{c}2 \\
5,3 \% \\
\end{array}$ & $\begin{array}{c}0 \\
0,0 \% \\
\end{array}$ & $\begin{array}{c}0 \\
0,0 \% \\
\end{array}$ & $\begin{array}{c}2 \\
3,5 \% \\
\end{array}$ & $\begin{array}{c}21 \\
3,9 \% \\
\end{array}$ & 1,000 \\
\hline
\end{tabular}




\subsubsection{Verlegungsrate und Verlegungsgründe}

16,1\% der Neugeborenen (4439 Fälle) mussten im Untersuchungszeitraum zur intensiven Betreuung auf eine neonatologische Station verlegt werden, wobei die Verlegungsrate mit dem BMI der Mutter anstieg. Der Anteil der Frauen, bei deren Kindern die Notwendigkeit einer Verlegung gesehen wurde, war unter übergewichtigen mit 17,5\% und adipösen Frauen mit 21,1\% signifikant höher (jeweils $\mathrm{p}<0,001$; Chi-Quadrat-Test) als unter Frauen des Referenzkollektivs mit 14,8\% (Tab. 69; Abb. 23).

Tab. 69 Absolute und relative Häufigkeiten einer kindlichen Verlegung auf die neonatologische Station in Bezug auf maternale BMI-Kategorien

BMI-Kategorien $\left[\mathrm{kg} / \mathrm{m}^{2}\right]$

\begin{tabular}{|c|c|c|c|c|c|c|c|}
\hline Verlegung & $\begin{array}{c}\mathbf{1 8 , 5 - 2 4 , 9} \\
n=18720\end{array}$ & $\begin{array}{c}\mathbf{2 5 , 0 - 2 9 , 9} \\
n=5718\end{array}$ & $\begin{array}{c}\mathbf{3 0 , 0 - 3 4 , 9} \\
n=1994\end{array}$ & $\begin{array}{c}\mathbf{3 5 , 0 - 3 9 , 9} \\
n=769\end{array}$ & $\begin{array}{c}\geq \mathbf{4 0} \\
n=417\end{array}$ & $\begin{array}{c}\geq \mathbf{3 0} \\
n=3180\end{array}$ & $\begin{array}{c}\text { Gesamt } \\
\mathrm{N}=27618\end{array}$ \\
\hline \multirow{2}{*}{$\mathrm{Ja}$} & 2766 & 1022 & ב421 & 154 & 96 & "671 & 44439 \\
\hline & $14,8 \%$ & $17,5 \%$ & $21,1 \%$ & $20,0 \%$ & $23,0 \%$ & $21,1 \%$ & $16,1 \%$ \\
\hline \multirow{2}{*}{ Nein } & 15954 & 4716 & 1573 & 615 & 321 & 2509 & 23179 \\
\hline & $85,2 \%$ & $82,5 \%$ & $78,9 \%$ & $80,0 \%$ & $77,0 \%$ & $78,9 \%$ & $83,9 \%$ \\
\hline
\end{tabular}

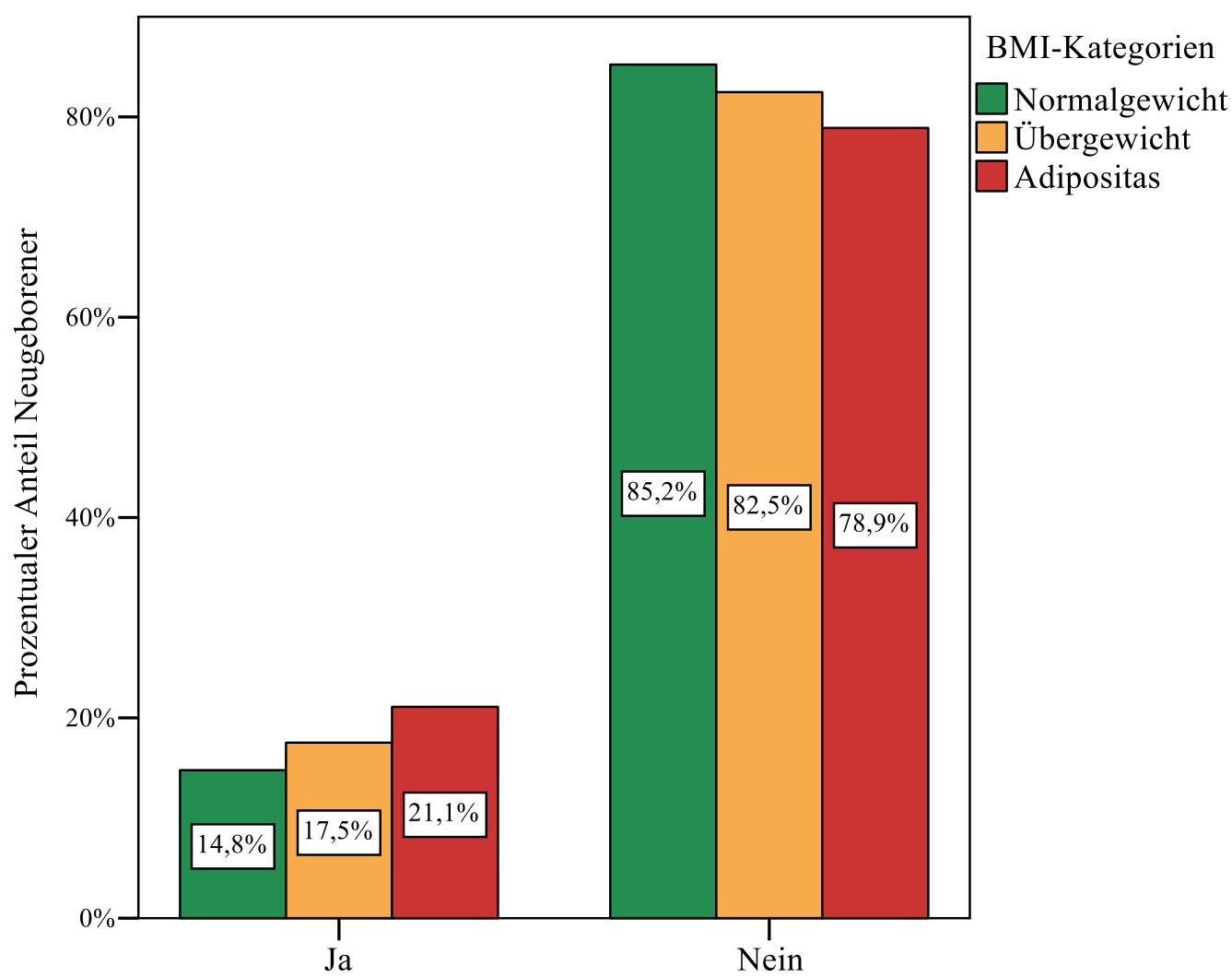

Verlegung auf neonatologische Station

Abb. 23 Relative Häufigkeitsverteilung einer kindlichen Verlegung auf die neonatologische Station in Bezug auf maternale BMI-Kategorien ( $N=27618)$ 
Tabelle 70 zeigt die absoluten und relativen Häufigkeiten der verschiedenen Indikationen zur kindlichen Verlegung auf eine neonatologische Station in Bezug auf die mütterlichen BMIKategorien. Da eventuell pro Kind mehrere Gründe für eine Verlegung sprachen, sind Mehrfachnennungen möglich. Die angegebenen p-Werte beziehen sich auf den Vergleich der Gewichtsgruppen Normalgewicht und Adipositas und wurden mithilfe des Chi-Quadrat-Tests bzw. des exakten Tests nach Fisher ermittelt.

In den BMI-Kategorien Normalgewicht und Adipositas wurde die Frühgeburtlichkeit mit 39,3\% bzw. 38,0\% als häufigste Verlegungsindikation genannt. Innerhalb der adipösen Frauen reihten sich die Indikationen Hypoglykämie bei Diabetes mellitus der Mutter (15,5\%), Atemnot bzw. Atemstörung (14,8\%), niedriges Geburtsgewicht $(13,1 \%)$ und Infektionen $(8,8 \%)$ an.

Der Verlegungsgrund Hypoglykämie bei Diabetes mellitus der Mutter wurde bei den Kindern von adipösen Frauen mit 15,5\% signifikant häufiger angegeben, als bei Kindern von normalgewichtigen Frauen mit 7,4\%.

Im Vergleich zu den Frauen mit einem BMI $\geq 30 \mathrm{~kg} / \mathrm{m}^{2}$ (8,8\%) gaben kindliche Infektionen in der Referenzgruppe (11,5\%) signifikant häufiger Anlass zur Verlegung auf die neonatologische Station ( $\mathrm{p}=0,047$ ). Beim Vergleich der Mütter mit einem BMI $\geq 40 \mathrm{~kg} / \mathrm{m}^{2}$ mit denen der Referenzgruppe trat diese Indikation mit 15,6\% gegenüber 11,5\% jedoch bei den Kindern der erstgenannten, stark adipösen Gruppe von Frauen prozentual häufiger auf $(p=0,210)$.

Bzgl. der bislang nicht erwähnten, in Tabelle 70 dargestellten Verlegungsindikationen konnten beim Vergleich der Patientinnen mit einem BMI zwischen 18,5 und 24,9 kg/m² und einem BMI $\geq 30 \mathrm{~kg} / \mathrm{m}^{2}$ keine signifikanten Unterschiede beobachtet werden.

Beim Vergleich der Kategorien Normalgewicht und Übergewicht fiel jedoch bei zwei Indikationen ein signifikanter Unterschied zwischen den Gruppen auf. Eine kindliche Verlegung aufgrund einer intrauterinen Hypoxie wurde mit 1,1\% (11 Fälle) signifikant häufiger bei übergewichtigen Frauen, als bei Normalgewichtigen mit 0,5\% (13 Fälle) verzeichnet ( $p=0,032)$. Auch der Verlegungsgrund Asphyxie unter der Geburt wurde bei den Neugeborenen übergewichtiger Frauen mit 0,6\% (6 Fälle) im Vergleich zum Referenzkollektiv mit 0,1\% (4 Fälle) signifikant häufiger angeführt $(\mathrm{p}=0,027)$. 
Tab. 70 Absolute und relative Häufigkeiten der verschiedenen Indikationen zur kindlichen Verlegung auf die neonatologische Station in Bezug auf maternale BMI-Kategorien

BMI-Kategorien $\left[\mathrm{kg} / \mathrm{m}^{2}\right]$

\begin{tabular}{|c|c|c|c|c|c|c|c|c|}
\hline $\begin{array}{l}\text { Verlegungs- } \\
\text { grund }\end{array}$ & $\begin{array}{c}\mathbf{1 8 , 5 - 2 4 , 9} \\
n=2766\end{array}$ & $\begin{array}{c}\mathbf{2 5 , 0 - 2 9 , 9} \\
\mathrm{n}=1002\end{array}$ & $\begin{array}{c}30,0-34,9 \\
n=421\end{array}$ & $\begin{array}{c}35,0-39,9 \\
n=154\end{array}$ & $\begin{array}{l}\geq \mathbf{4 0} \\
\mathrm{n}=96\end{array}$ & $\begin{array}{c}\geq \mathbf{3 0} \\
\mathrm{n}=671\end{array}$ & $\begin{array}{l}\text { Gesamt } \\
\mathrm{N}=4439\end{array}$ & P-Wert \\
\hline Frühgeburt- & 1086 & 361 & 175 & 53 & 27 & 255 & 1702 & \\
\hline lichkeit & $39,3 \%$ & $36,0 \%$ & $41,5 \%$ & $34,4 \%$ & $28,8 \%$ & $38,0 \%$ & $38,4 \%$ & 0,548 \\
\hline Atemnot bzw. & 400 & 152 & 59 & 28 & 12 & 99 & 651 & \\
\hline Atemstörung & $14,5 \%$ & $15,2 \%$ & $14,0 \%$ & $18,2 \%$ & $12,5 \%$ & $14,8 \%$ & $14,7 \%$ & 0,847 \\
\hline niedriges Ge- & 407 & 129 & 51 & 22 & 15 & 88 & 624 & \\
\hline burtsgewicht & $14,7 \%$ & $12,9 \%$ & $12,1 \%$ & $14,3 \%$ & $15,6 \%$ & $13,1 \%$ & $14,1 \%$ & 0,290 \\
\hline \multirow{2}{*}{ Infektionen } & 317 & 114 & 37 & 7 & 15 & 59 & 490 & \multirow[b]{2}{*}{0,047} \\
\hline & $11,5 \%$ & $11,4 \%$ & $8,8 \%$ & $4,5 \%$ & $15,6 \%$ & $8,8 \%$ & $11,0 \%$ & \\
\hline Hypoglykämie & 204 & 104 & 62 & 24 & 18 & 104 & 412 & \\
\hline ei DM Mutter & $7,4 \%$ & $10,4 \%$ & $14,7 \%$ & $15,6 \%$ & $18,8 \%$ & $15,5 \%$ & $9,3 \%$ & $<0,001$ \\
\hline \multirow{2}{*}{ Fehlbildung } & 192 & 87 & 17 & 12 & 5 & 34 & 313 & \multirow[b]{2}{*}{0,106} \\
\hline & $6,9 \%$ & $8,7 \%$ & $4,0 \%$ & $7,8 \%$ & $5,2 \%$ & $5,1 \%$ & $7,1 \%$ & \\
\hline \multirow{2}{*}{ Ikterus } & 133 & 56 & 15 & 6 & 3 & 24 & 213 & \multirow[b]{2}{*}{0,170} \\
\hline & $4,8 \%$ & $5,6 \%$ & $3,6 \%$ & $3,9 \%$ & $3,1 \%$ & $3,6 \%$ & $4,8 \%$ & \\
\hline \multirow{2}{*}{$\begin{array}{l}\text { Noxen (Plazen- } \\
\text { ta/Muttermilch) }\end{array}$} & 127 & 34 & 17 & 5 & 1 & 23 & 184 & \multirow[b]{2}{*}{0,186} \\
\hline & $4,6 \%$ & $3,4 \%$ & $4,0 \%$ & $3,2 \%$ & $1,0 \%$ & $3,4 \%$ & $4,1 \%$ & \\
\hline \multirow{2}{*}{$\begin{array}{l}\text { Intraut. Mangel- } \\
\text { entwicklung }\end{array}$} & 66 & 27 & 7 & 2 & 1 & 10 & 103 & \multirow[b]{2}{*}{0,157} \\
\hline & $2,4 \%$ & $2,7 \%$ & $1,7 \%$ & $1,3 \%$ & $1,0 \%$ & $1,5 \%$ & $2,3 \%$ & \\
\hline \multirow{2}{*}{$\begin{array}{l}\text { Erkrankung } \\
\text { der Mutter }\end{array}$} & 52 & 22 & 10 & 4 & 1 & 15 & 89 & \multirow[b]{2}{*}{0,550} \\
\hline & $1,9 \%$ & $2,2 \%$ & $2,4 \%$ & $2,6 \%$ & $1,0 \%$ & $2,2 \%$ & $2,0 \%$ & \\
\hline \multirow{2}{*}{$\begin{array}{l}\text { SW-Kompli- } \\
\text { kationen } \\
\end{array}$} & 32 & 17 & 8 & 1 & 2 & 11 & 60 & \multirow[b]{2}{*}{0,313} \\
\hline & $1,2 \%$ & $1,7 \%$ & $1,9 \%$ & $0,6 \%$ & $2,1 \%$ & $1,6 \%$ & $1,4 \%$ & \\
\hline \multirow{2}{*}{ Azidose } & 25 & 8 & 3 & 2 & 1 & 6 & 39 & \multirow{3}{*}{0,981} \\
\hline & $0,9 \%$ & $0,8 \%$ & $0,7 \%$ & $1,3 \%$ & $1,0 \%$ & $0,9 \%$ & $0,9 \%$ & \\
\hline Intrauterine & 13 & 11 & 1 & 2 & 1 & 4 & 28 & \\
\hline ypoxie & $0,5 \%$ & $1,1 \%$ & $0,2 \%$ & $1,3 \%$ & $1,0 \%$ & $0,6 \%$ & $0,6 \%$ & 0,758 \\
\hline \multirow{2}{*}{ Geburtstrauma } & 16 & 3 & 3 & 4 & 1 & 8 & 27 & \multirow[b]{2}{*}{0,115} \\
\hline & $0,6 \%$ & $0,3 \%$ & $0,7 \%$ & $2,6 \%$ & $1,0 \%$ & $1,2 \%$ & $0,6 \%$ & \\
\hline \multirow{2}{*}{$\begin{array}{l}\text { Mekonium- } \\
\text { Aspiration }\end{array}$} & 12 & 4 & 1 & 2 & 1 & 4 & 20 & \multirow[b]{2}{*}{0,533} \\
\hline & $0,4 \%$ & $0,4 \%$ & $0,2 \%$ & $1,3 \%$ & $1,0 \%$ & $0,6 \%$ & $0,5 \%$ & \\
\hline \multirow{2}{*}{$\begin{array}{l}\text { Sonstige Aspi- } \\
\text { rationssyndrom }\end{array}$} & 13 & 5 & 1 & 4 & 1 & 6 & 24 & \multirow[b]{2}{*}{0,239} \\
\hline & $0,5 \%$ & $0,5 \%$ & $0,2 \%$ & $2,6 \%$ & $1,0 \%$ & $0,9 \%$ & $0,5 \%$ & \\
\hline \multirow{2}{*}{$\begin{array}{l}\text { Asphyxie unter } \\
\text { der Geburt }\end{array}$} & 4 & 6 & 2 & 0 & 0 & 2 & 12 & \multirow[b]{2}{*}{0,333} \\
\hline & $0,1 \%$ & $0,6 \%$ & $0,5 \%$ & $0,0 \%$ & $0,0 \%$ & $0,3 \%$ & $0,3 \%$ & \\
\hline
\end{tabular}




\subsubsection{Perinatale Mortalität}

Die perinatale Mortalität, die die Fälle von intrauterinem Fruchttod sowie subpartual oder neonatal verstorbenen Kindern zusammenfasst, betrug im Untersuchungszeitraum mit insgesamt 180 dokumentierten Fällen 0,6\%.

Unter den Kindern adipöser Frauen war sie mit 1,0\% signifikant höher als unter denen normalgewichtiger Frauen mit 0,6\% (p=0,006; Chi-Quadrat-Test). Der Anteil der Frauen, deren Kinder perinatal verstarben, war unter den Patientinnen mit einem BMI $\geq 40 \mathrm{~kg} / \mathrm{m}^{2}$ mit 1,2\% am größten und damit doppelt so hoch wie unter den Frauen des Referenzkollektivs (Tab. 71).

Tab. 71 Absolute und relative Häufigkeiten der perinatalen Mortalität in Bezug auf maternale BMI-Kategorien BMI-Kategorien $\left[\mathrm{kg} / \mathrm{m}^{2}\right]$

\begin{tabular}{|c|c|c|c|c|c|c|c|}
\hline $\begin{array}{l}\text { Perinatale } \\
\text { Mortalität }\end{array}$ & $\begin{array}{c}\mathbf{1 8 , 5 - 2 4 , 9} \\
n=18806\end{array}$ & $\begin{array}{c}\mathbf{2 5 , 0 - 2 9 , 9} \\
n=5738\end{array}$ & $\begin{array}{c}\mathbf{3 0 , 0 - 3 4 , 9} \\
n=2009\end{array}$ & $\begin{array}{c}\mathbf{3 5 , 0 - 3 9 , 9} \\
\mathrm{n}=774\end{array}$ & $\begin{array}{c}\geq \mathbf{4 0} \\
n=420\end{array}$ & $\begin{array}{c}\geq \mathbf{3 0} \\
\mathrm{n}=3203\end{array}$ & $\begin{array}{c}\text { Gesamt } \\
\mathrm{N}=27747\end{array}$ \\
\hline \multirow{2}{*}{$\mathrm{Ja}$} & $\bar{~} 113$ & 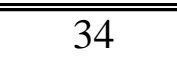 & $\overline{22}$ & $\bar{~} 6$ & $\overline{5}$ & $\bar{~} 33$ & $\bar{~} 180$ \\
\hline & $0,6 \%$ & $0,6 \%$ & $1,1 \%$ & $0,8 \%$ & $1,2 \%$ & $1,0 \%$ & $0,6 \%$ \\
\hline \multirow{2}{*}{ Nein } & 18693 & 5704 & 1987 & 768 & 415 & 3170 & 27567 \\
\hline & $99,4 \%$ & $99,4 \%$ & $98,9 \%$ & $99,2 \%$ & $98,8 \%$ & $99,0 \%$ & $99,4 \%$ \\
\hline
\end{tabular}

Insgesamt waren im Zeitraum der Analyse 79 Schwangerschaften (0,3\%) von einem intrauterinen Fruchttod betroffen (Tab. 72). Dabei wurde unter Durchführung des Chi-Quadrat-Tests eine signifikante Häufung der Fälle bei adipösen Schwangeren (16 Fälle, 0,5\%) im Vergleich zum Referenzkollektiv (51 Fälle, 0,3\%) verzeichnet ( $\mathrm{p}=0,030)$.

Die Betrachtung in Abhängigkeit des Gestationsalters zeigte, dass die Mehrzahl der Fälle eines IUFT bei einem Schwangerschaftsalter $<37+0$ SSW auftraten (55 Fälle, 69,7\%), während 20 Fälle $(25,3 \%)$ bei einem Gestationsalter zwischen $37+0$ und 39+6 SSW und 4 Fälle $(5,1 \%)$ bei einer Tragzeit $\geq 40+0$ SSW zu finden waren (Tab. 75).

Tab. 72 Absolute und relative Häufigkeiten eines intrauterinen Fruchttodes in Bezug auf maternale BMI-Kategorien

BMI-Kategorien $\left[\mathrm{kg} / \mathrm{m}^{2}\right]$

\begin{tabular}{|c|c|c|c|c|c|c|c|}
\hline IUFT & $\begin{array}{l}\mathbf{1 8 , 5 - 2 4 , 9} \\
\mathrm{n}=18806\end{array}$ & $\begin{array}{c}\mathbf{2 5 , 0 - 2 9 , 9} \\
n=5738\end{array}$ & $\begin{array}{c}\mathbf{3 0 , 0 - 3 4 , 9} \\
\mathrm{n}=2009\end{array}$ & $\begin{array}{c}\mathbf{3 5 , 0 - 3 9 , 9} \\
n=774\end{array}$ & $\begin{array}{c}\geq \mathbf{4 0} \\
\mathrm{n}=420\end{array}$ & $\begin{array}{c}\geq \mathbf{3 0} \\
n=3203\end{array}$ & $\begin{array}{c}\text { Gesamt } \\
\mathrm{N}=27747\end{array}$ \\
\hline \multirow{2}{*}{$\mathrm{Ja}$} & $\overline{51}$ & 12 & $\overline{111}$ & $\overline{3}$ & 2 & 16 & $\overline{79}$ \\
\hline & $0,3 \%$ & $0,2 \%$ & $0,5 \%$ & $0,4 \%$ & $0,5 \%$ & $0,5 \%$ & $0,3 \%$ \\
\hline \multirow[t]{2}{*}{ Nein } & 18755 & 5726 & 1998 & 771 & 418 & 3187 & 27668 \\
\hline & $99,7 \%$ & $99,8 \%$ & $99,5 \%$ & $99,6 \%$ & $99,5 \%$ & $99,5 \%$ & $99,7 \%$ \\
\hline
\end{tabular}


Zum Tod des Fetus sub partu kam es im Untersuchungszeitraum bei insgesamt 42 Geburten $(0,2 \%)$, wobei das Gestationsalter der verstorbenen Kinder in 34 Fällen $(80,9 \%)<37+0$ SSW betrug, wovon wiederum 25 Fälle $(59,5 \%)$ auf eine Tragzeit $<27+0$ SSW fielen. In 6 Fällen $(14,3 \%)$ wurde der subpartuale Tod bei einem Schwangerschaftsalter zwischen 37+0 und 39+6 SSW verzeichnet, während 2 Kinder $(4,8 \%)$ mit einem Gestationsalter $\geq 40+0$ SSW während der Geburt verstarben (Tab. 73 und 75).

In Bezug auf den mütterlichen BMI konnten im Chi-Quadrat-Test keine signifikanten Unterschiede zwischen den Gewichtsklassen Normalgewicht, Übergewicht und Adipositas festgestellt werden ( $\mathrm{p}=0,520$ bzw. p=0,968). Das Ereignis wurde bei der Geburt von 0,2\% (30 Fälle) der normalgewichtigen, 0,1\% (7 Fälle) der übergewichtigen und 0,2\% (5 Fälle) der adipösen Frauen dokumentiert. Bei diesen Analysen waren die Fälle des intrauterinen Fruchttodes (n=79) von der untersuchten Stichprobe ausgeschlossen.

Tab. 73 Absolute und relative Häufigkeiten eines subpartualen Todes in Bezug auf maternale BMI-Kategorien

BMI-Kategorien $\left[\mathrm{kg} / \mathrm{m}^{2}\right]$

\begin{tabular}{|c|c|c|c|c|c|c|c|}
\hline $\begin{array}{l}\text { Subpartual } \\
\text { verstorben }\end{array}$ & $\begin{array}{c}\mathbf{1 8 , 5 - 2 4 , 9} \\
\mathrm{n}=18755\end{array}$ & $\begin{array}{c}\mathbf{2 5 , 0 - 2 9 , 9} \\
n=5726\end{array}$ & $\begin{array}{c}\mathbf{3 0 , 0 - 3 4 , 9} \\
n=1998\end{array}$ & $\begin{array}{c}\mathbf{3 5 , 0 - 3 9 , 9} \\
n=771\end{array}$ & $\begin{array}{c}\geq \mathbf{4 0} \\
\mathrm{n}=418\end{array}$ & $\begin{array}{c}\geq \mathbf{3 0} \\
n=3187\end{array}$ & $\begin{array}{c}\text { Gesamt } \\
\mathrm{N}=27668\end{array}$ \\
\hline \multirow{2}{*}{$\mathrm{Ja}$} & 30 & $\overline{77}$ & 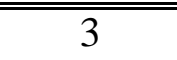 & 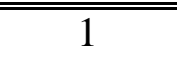 & 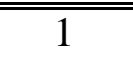 & 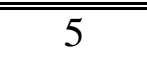 & $\overline{442}$ \\
\hline & $0,2 \%$ & $0,1 \%$ & $0,2 \%$ & $0,1 \%$ & $0,2 \%$ & $0,2 \%$ & $0,2 \%$ \\
\hline \multirow[t]{2}{*}{ Nein } & 18725 & 5719 & 1995 & 770 & 417 & 3182 & 27626 \\
\hline & $99,8 \%$ & $99,9 \%$ & $99,8 \%$ & $99,9 \%$ & $99,8 \%$ & $99,8 \%$ & $99,8 \%$ \\
\hline
\end{tabular}

Hinsichtlich eines neonatalen Todes innerhalb der ersten sieben Lebenstage wurden im Analysezeitraum insgesamt 59 Ereignisse (0,2\%) verzeichnet (Tab. 74). Unter Durchführung des ChiQuadrat-Tests konnte gezeigt werden, dass der Anteil der Frauen, deren Kinder in den ersten sieben Lebenstagen verstarben, unter adipösen Patientinnen mit 0,4\% (12 Fälle) signifikant höher war $(\mathrm{p}=0,016)$ als unter den normalgewichtigen mit 0,2\% (32 Fälle). Bei diesen Auswertungen wurden die Fälle totgeborener Kinder $(n=121)$ vom untersuchten Kollektiv ausgeschlossen und lediglich die Lebendgeburten berücksichtigt.

88,1\% der neonatalen Todesfälle fanden bei einem Gestationsalter <37+0 SSW statt, während die restlichen 11,9\% auf eine Gestationsalter zwischen 37+0 und 39+6 SSW fielen (Tab. 75). 
Tab. 74 Absolute und relative Häufigkeiten eines neonatalen Todes innerhalb der ersten sieben Lebenstage in Bezug auf maternale BMI-Kategorien

\begin{tabular}{lccccccc} 
& \multicolumn{7}{c}{ BMI-Kategorien $\left[\mathrm{kg} / \mathrm{m}^{2}\right]$} \\
neonataler \\
\cline { 2 - 7 } Tod & $\mathbf{1 8 , 5 - 2 4 , 9}$ & $\mathbf{2 5 , 0 - 2 9 , 9}$ & $\mathbf{3 0 , 0 - 3 4 , 9}$ & $\mathbf{3 5 , 0 - 3 9 , 9}$ & $\mathbf{2 4 0}$ & $\mathbf{2 3 0}$ & Gesamt \\
\hline \hline \multirow{2}{*}{ Ja } & $\mathrm{n}=18722$ & $\mathrm{n}=5719$ & $\mathrm{n}=1994$ & $\mathrm{n}=770$ & $\mathrm{n}=417$ & $\mathrm{n}=3181$ & $\mathrm{~N}=27622$ \\
\hline \multirow{2}{*}{ Nein } & $0,2 \%$ & $0,3 \%$ & $0,4 \%$ & $0,3 \%$ & $0,5 \%$ & $0,4 \%$ & $0,2 \%$ \\
\hline
\end{tabular}

Tab. 75 Absolute und relative Häufigkeiten der perinatalen Mortalität in Bezug auf das Gestationsalter Gestationsalter [SSW]

\begin{tabular}{|c|c|c|c|c|c|c|}
\hline \multirow[b]{2}{*}{$\begin{array}{l}\text { Perinatale } \\
\text { Mortalität }\end{array}$} & \multicolumn{5}{|c|}{ Gestationsalter [SSW] } & \multirow[b]{2}{*}{ Gesamt } \\
\hline & $\geq 40+0$ & $37+0-39+6$ & $32+0-36+6$ & $27+0-31+6$ & $<27+0$ & \\
\hline \multirow{2}{*}{ IUFT } & $\overline{44}$ & 20 & 30 & 13 & 12 & 79 \\
\hline & $5,1 \%$ & $25,3 \%$ & $38,0 \%$ & $16,5 \%$ & $15,2 \%$ & $100 \%$ \\
\hline$\overline{\text { Subpartual }}$ & 2 & 6 & 5 & 4 & 25 & 42 \\
\hline verstorben & $4,8 \%$ & $14,3 \%$ & $11,9 \%$ & $9,5 \%$ & $59,5 \%$ & $100 \%$ \\
\hline Neonataler & 0 & 7 & 20 & 10 & 22 & 59 \\
\hline Tod & $0,0 \%$ & $11,9 \%$ & $33,9 \%$ & $16,9 \%$ & $37,3 \%$ & $100 \%$ \\
\hline
\end{tabular}




\subsection{Wochenbettverlauf}

\subsubsection{Stillen}

Insgesamt stillten 77,6\% der Wöchnerinnen ihre Kinder voll, 3,8\% pumpten die Muttermilch ab, 1,3\% der Frauen stillten zum Teil und 17,4\% der Mütter entschieden sich dazu, ihre Kinder nicht zu stillen (Tab. 76; Abb. 24).

In Bezug auf die mütterliche BMI-Kategorie konnten signifikante Unterschiede im Stillverhalten zwischen den verschiedenen Gewichtsklassen festgestellt werden.

Die Kinder von übergewichtigen und adipösen Frauen wurden mit 74,9\% bzw. 67,7\% signifikant seltener voll gestillt, als die Neugeborenen normalgewichtiger Frauen mit 80,0\%. Dabei nahm die Rate der voll stillenden Mütter mit zunehmendem BMI ab und lag bei Wöchnerinnen der Kategorie Adipositas Grad III bei einem Minimum von 60,4\%.

Umgekehrt verhielt es sich hinsichtlich der nicht-stillenden Mütter, deren Anteil mit zunehmendem BMI stieg und unter übergewichtigen und adipösen Wöchnerinnen mit 19,8\% bzw. 25,8\% signifikant höher war als unter den Frauen des Referenzkollektivs mit 15,3\%.

Der Anteil der Frauen, die ihre Muttermilch abpumpten, war in der BMI-Gruppe Adipositas mit 5,0\% signifikant höher als in der Kategorie Normalgewicht mit 3,5\%.

Der Chi-Quadrat-Test bestätigte mit $\mathrm{p}<0,001$ in allen Punkten statistische Signifikanz.

Tab. 76 Absolute und relative Häufigkeiten der verschiedenen Formen des Stillverhaltens der Wöchnerinnen in Bezug auf deren BMI-Kategorien

BMI-Kategorien $\left[\mathrm{kg} / \mathrm{m}^{2}\right]$

\begin{tabular}{|c|c|c|c|c|c|c|c|}
\hline Stillen & $\begin{array}{c}\mathbf{1 8 , 5}-24,9 \\
n=16160\end{array}$ & $\begin{array}{c}\mathbf{2 5 , 0 - 2 9 , 9} \\
n=4868\end{array}$ & $\begin{array}{c}\mathbf{3 0 , 0 - 3 4 , 9} \\
\mathrm{n}=1670\end{array}$ & $\begin{array}{c}\mathbf{3 5 , 0 - 3 9 , 9} \\
n=633\end{array}$ & $\begin{array}{c}\geq 40 \\
n=341\end{array}$ & $\begin{array}{c}\geq \mathbf{3 0} \\
\mathrm{n}=2644\end{array}$ & $\begin{array}{c}\text { Gesamt } \\
\mathrm{N}=23672\end{array}$ \\
\hline \multirow{2}{*}{ Voll } & 12922 & 3646 & 11173 & 412 & 206 & 1791 & 18359 \\
\hline & $80,0 \%$ & $74,9 \%$ & $70,2 \%$ & $65,1 \%$ & $60,4 \%$ & $67,7 \%$ & $77,6 \%$ \\
\hline \multirow{2}{*}{$\begin{array}{l}\text { Mutter } \\
\text { pumpt ab }\end{array}$} & 561 & 196 & 81 & 37 & 14 & 132 & 889 \\
\hline & $3,5 \%$ & $4,0 \%$ & $4,9 \%$ & $5,8 \%$ & $4,1 \%$ & $5,0 \%$ & $3,8 \%$ \\
\hline \multirow{2}{*}{ Zum Teil } & 199 & 61 & 21 & 13 & 6 & 40 & 300 \\
\hline & $1,2 \%$ & $1,3 \%$ & $1,3 \%$ & $2,1 \%$ & $1,8 \%$ & $1,5 \%$ & $1,3 \%$ \\
\hline \multirow{2}{*}{ Nein } & 2478 & 965 & 395 & 171 & 115 & 681 & 4124 \\
\hline & $15,3 \%$ & $19,8 \%$ & $23,7 \%$ & $27,0 \%$ & $33,7 \%$ & $25,8 \%$ & $17,4 \%$ \\
\hline
\end{tabular}




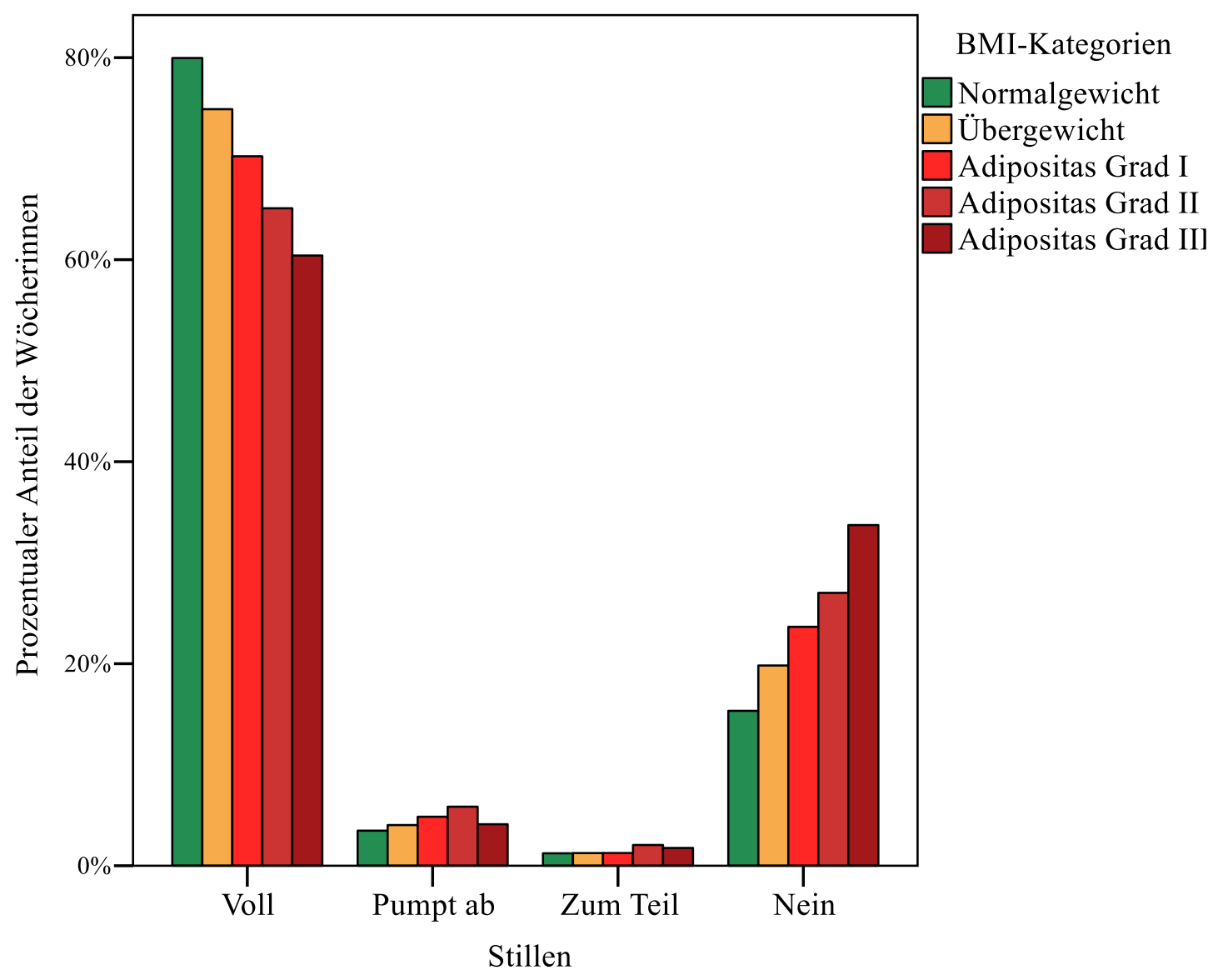

Abb. 24 Relative Häufigkeitsverteilung der verschiedenen Formen des Stillverhaltens der Wöchnerinnen in Bezug auf deren BMI-Kategorien $(\mathrm{N}=23672)$

\subsubsection{Wundheilungsstörungen}

Eine revisionsbedürftige Wundheilungsstörung trat im Untersuchungszeitraum in insgesamt 42 Fällen $(0,1 \%)$ auf und wurde bei übergewichtigen (10 Fälle) und adipösen Wöchnerinnen (8 Fälle) mit jeweils 0,2\% prozentual etwas häufiger diagnostiziert, als bei den Frauen des Referenzkollektivs mit 0,1\% (24 Fälle). Die Unterschiede zwischen den BMI-Gruppen zeigten jeweils mit $\mathrm{p}=0,406$ bzw. $\mathrm{p}=0,125$ keine statistische Signifikanz (Chi-Quadrat-Test).

Unter den Frauen, die per Kaiserschnitt entbanden ( $\mathrm{N}=6532)$, infizierte sich die Operationswunde in insgesamt 12 Fällen (0,2\%). Das Ereignis wurde bei adipösen Patientinnen mit 0,4\% (4 Fälle) öfter festgestellt, als bei normalgewichtigen Frauen mit 0,2\% (6 Fälle), wobei der ChiQuadrat-Test ebenfalls keine statistische Signifikanz bestätigte $(\mathrm{p}=0,236)$. 
Im Vergleich zur Referenzgruppe wurde bei Wöchnerinnen der BMI-Gruppe Adipositas Grad III jedoch sowohl die revisionsbedürftige Wundheilungsstörung mit 0,7\% (3 Fälle) als auch darunter die Wundinfektion nach Sectio mit 1,2\% (2 Fälle) signifikant häufiger beobachtet ( $\mathrm{p}=0,021$ bzw. $\mathrm{p}=0,039$; exakter Test nach Fisher).

Die absoluten und relativen Häufigkeiten einer revisionsbedürftigen Wundheilungsstörung insgesamt sowie einer Wundinfektion nach Sectio für sich betrachtet sind in Tabellen 77 und 78 dargestellt.

Tab. 77 Absolute und relative Häufigkeiten einer revisionsbedürftigen Wundheilungsstörung Bezug auf maternale BMI-Kategorien

BMI-Kategorien $\left[\mathrm{kg} / \mathrm{m}^{2}\right]$

\begin{tabular}{|c|c|c|c|c|c|c|c|}
\hline \multirow{3}{*}{$\begin{array}{l}\text { Wundhei- } \\
\text { lungsstörung }\end{array}$} & \multicolumn{6}{|c|}{ 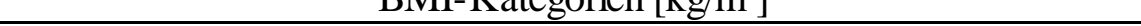 } & \multirow{3}{*}{$\begin{array}{c}\text { Gesamt } \\
\mathrm{N}=27736\end{array}$} \\
\hline & $18,5-24,9$ & $25,0-29,9$ & $30,0-34,9$ & $35,0-39,9$ & $\geq 40$ & $\geq \mathbf{3 0}$ & \\
\hline & $\mathrm{n}=18798$ & $\mathrm{n}=5736$ & $\mathrm{n}=2009$ & $\mathrm{n}=773$ & $\mathrm{n}=420$ & $n=3202$ & \\
\hline \multirow{2}{*}{$\mathrm{Ja}$} & 24 & 10 & 5 & 0 & 3 & 8 & 42 \\
\hline & $0,1 \%$ & $0,2 \%$ & $0,2 \%$ & $0,0 \%$ & $0,7 \%$ & $0,2 \%$ & $0,1 \%$ \\
\hline \multirow{2}{*}{ Nein } & 18774 & 5726 & 2004 & 773 & 417 & 3195 & 27694 \\
\hline & $99,9 \%$ & $99,8 \%$ & $99,8 \%$ & $100,0 \%$ & $99,3 \%$ & $99,8 \%$ & $99,9 \%$ \\
\hline
\end{tabular}

Tab. 78 Absolute und relative Häufigkeiten einer Wundinfektion nach Sectio in Bezug auf maternale BMI-Kategorien

BMI-Kategorien $\left[\mathrm{kg} / \mathrm{m}^{2}\right]$

\begin{tabular}{|c|c|c|c|c|c|c|c|}
\hline $\begin{array}{l}\text { Wundinfektion } \\
\text { nach Sectio }\end{array}$ & $\begin{array}{c}\mathbf{1 8 , 5 - 2 4 , 9} \\
\mathrm{n}=3977\end{array}$ & $\begin{array}{c}\mathbf{2 5 , 0 - 2 9 , 9} \\
\mathrm{n}=1475\end{array}$ & $\begin{array}{c}\mathbf{3 0 , 0 - 3 4 , 9} \\
n=629\end{array}$ & $\begin{array}{c}\mathbf{3 5 , 0 - 3 9 , 9} \\
\mathrm{n}=283\end{array}$ & $\begin{array}{c}\geq 40 \\
\mathrm{n}=168\end{array}$ & $\begin{array}{c}\geq \mathbf{3 0} \\
\mathrm{n}=1080\end{array}$ & $\begin{array}{r}\text { Gesamt } \\
\mathrm{N}=6532\end{array}$ \\
\hline & 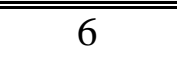 & $\bar{~} 2$ & 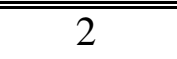 & 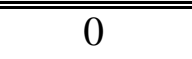 & $\bar{~} 2$ & 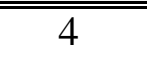 & $\overline{12}$ \\
\hline $\mathrm{Ja}$ & $0,2 \%$ & $0,1 \%$ & $0,3 \%$ & $0,0 \%$ & $1,2 \%$ & $0,4 \%$ & $0,2 \%$ \\
\hline Nein & $\begin{array}{c}3971 \\
99,8 \%\end{array}$ & $\begin{array}{c}1473 \\
99,9 \%\end{array}$ & $\begin{array}{c}627 \\
99,7 \%\end{array}$ & $\begin{array}{c}283 \\
100,0 \%\end{array}$ & $\begin{array}{c}166 \\
98.8 \%\end{array}$ & $\begin{array}{c}1076 \\
99,6 \%\end{array}$ & $\begin{array}{c}6520 \\
99,8 \%\end{array}$ \\
\hline
\end{tabular}




\subsubsection{Fieber post partum}

166 Mütter (0,6\%) entwickelten im Wochenbett eine Körpertemperatur über $38{ }^{\circ} \mathrm{C}$, die länger als zwei Tage anhielt. Dabei war eine Assoziation mit dem mütterlichen BMI im Sinne einer Zunahme der Fälle mit steigender BMI-Kategorie von Normalgewicht $(0,5 \%)$ bis Adipositas Grad III (2,1\%) festzustellen (Tab. 79). Adipöse Wöchnerinnen in ihrer Gesamtheit entwickelten mit 1,2\% signifikant häufiger postpartales Fieber im Vergleich zu den normalgewichtigen Frauen mit 0,5\% ( $\mathrm{p}<0,001$; Chi-Quadrat-Test).

Tab. 79 Absolute und relative Häufigkeiten von postpartalem Fieber in Bezug auf maternale BMI-Kategorien BMI-Kategorien $\left[\mathrm{kg} / \mathrm{m}^{2}\right]$

\begin{tabular}{lccccccc} 
Fieber post & $\mathbf{1 8 , 5 - 2 4 , 9}$ & $\mathbf{2 5 , 0 - 2 9 , 9}$ & $\mathbf{3 0 , 0 - 3 4 , 9}$ & $\mathbf{3 5 , 0 - 3 9 , 9}$ & $\mathbf{2 4 0}$ & $\mathbf{2} \mathbf{3 0}$ & Gesamt \\
partum & $\mathrm{n}=18797$ & $\mathrm{n}=5736$ & $\mathrm{n}=2009$ & $\mathrm{n}=773$ & $\mathrm{n}=420$ & $\mathrm{n}=3202$ & $\mathrm{~N}=27735$ \\
\hline \hline \multirow{2}{*}{ Ja } & 96 & 33 & 18 & 10 & 9 & 37 & 166 \\
& $0,5 \%$ & $0,6 \%$ & $0,9 \%$ & $1,3 \%$ & $2,1 \%$ & $1,2 \%$ & $0,6 \%$ \\
\hline \multirow{2}{*}{ Nein } & 18701 & 5703 & 1991 & 763 & 411 & 3165 & 27569 \\
& $99,5 \%$ & $99,4 \%$ & $99,1 \%$ & $98,7 \%$ & $97,9 \%$ & $98,8 \%$ & $99,4 \%$ \\
\hline
\end{tabular}

\subsubsection{Anämie}

Zwischen dem BMI der Frau und der Häufigkeit einer Anämie im Wochenbett konnte im ChiQuadrat-Test kein signifikanter Zusammenhang festgestellt werden, wobei insgesamt 18,5\% der Wöchnerinnen von einer Blutarmut mit einem Hämoglobinwert kleiner $10 \mathrm{~g} / \mathrm{dl}$ (6,2 mmol/l) betroffen waren (Tab. 80). Der Anteil war unter normalgewichtigen Frauen mit 18,6\% etwas höher als unter adipösen Wöchnerinnen mit 17,6\% ( $\mathrm{p}=0,197)$.

Tab. 80 Absolute und relative Häufigkeiten einer Anämie im Wochenbett in Bezug auf maternale BMI-Kategorien BMI-Kategorien $\left[\mathrm{kg} / \mathrm{m}^{2}\right]$

\begin{tabular}{lccccccc}
\cline { 2 - 6 } Anämie & $\begin{array}{c}\mathbf{1 8 , 5 - 2 4 , 9} \\
\mathrm{n}=18798\end{array}$ & $\begin{array}{c}\mathbf{2 5 , 0 - 2 9 , 9} \\
\mathrm{n}=5736\end{array}$ & $\begin{array}{c}\mathbf{3 0 , 0 - 3 4 , 9} \\
\mathrm{n}=2009\end{array}$ & $\begin{array}{c}\mathbf{3 5 , 0 - 3 9 , 9} \\
\mathrm{n}=773\end{array}$ & $\begin{array}{c}\mathbf{2} \mathbf{4 0} \\
\mathrm{n}=420\end{array}$ & $\begin{array}{c}\mathbf{2 3 0} \\
\mathrm{n}=3202\end{array}$ & $\begin{array}{c}\text { Gesamt } \\
\mathrm{N}=27736\end{array}$ \\
\hline \multirow{2}{*}{ Ja } & 3491 & 1076 & 356 & 128 & 80 & 564 & 5131 \\
& $18,6 \%$ & $18,8 \%$ & $17,7 \%$ & $16,6 \%$ & $19,0 \%$ & $17,6 \%$ & $18,5 \%$ \\
\hline \multirow{2}{*}{ Nein } & 15307 & 4660 & 1653 & 645 & 340 & 2638 & 22605 \\
& $81,4 \%$ & $81,2 \%$ & $82,3 \%$ & $83,4 \%$ & $81,0 \%$ & $82,4 \%$ & $81,5 \%$ \\
\hline
\end{tabular}




\section{DISKUSSION}

\subsection{Adipositas-Prävalenz}

Bezogen auf den maternalen BMI nach Angabe im Mutterpass waren im Untersuchungszeitraum von 2000 bis 2015 in der Klinik St. Hedwig insgesamt 4,9\% der Patientinnen untergewichtig, 64,5\% normalgewichtig, 19,7\% übergewichtig und 11,0\% adipös. Unter den Frauen mit einem BMI $\geq 30 \mathrm{~kg} / \mathrm{m}^{2}$ lag die Prävalenz von Adipositas Grad I, II und III jeweils bei 6,9\%, 2,7\% und 1,4\%. Innerhalb des Analysezeitraums war eine Zunahme der Prävalenz von Übergewicht und Adipositas zu verzeichnen. Der Anteil adipöser Schwangerer stieg ausgehend von einem Minimum von 9,1\% im Jahr 2004 auf den Höchstwert von 13,1\% im Jahr 2015 an. Die Rate übergewichtiger Patientinnen schwankte zwischen dem niedrigsten Wert von $16,4 \%$ im Jahr 2002 und dem Maximum von 21,3\% im Jahr 2015.

Beim Vergleich verschiedener Studien hinsichtlich der Prävalenz von Adipositas in der Schwangerschaft gilt es, Zeitraum und Ort der Datenerhebungen zu berücksichtigen, da zum einen die globale Adipositas-Prävalenz in den letzten Jahren angestiegen ist und zum anderen internationale Unterschiede der allgemeinen Häufigkeit bestehen (siehe 1.1.2). Sehr hohe Prävalenzen von Adipositas unter schwangeren Frauen wurden in einer US-amerikanischen Untersuchung im Zeitraum von 2007 bis 2008 mit 26,2\% (24) und in einer von 2002 bis 2007 durchgeführten englischen Studie mit 15,8\% (29) angegeben. In einer Publikation aus Australien lag der Anteil adipöser Schwangerer im Zeitraum von 1998 bis 2002 bei 13,5\% (32), während die Beobachtungen einer kanadischen Studie von 2004 bis 2012 einen Wert von 12,0\% ergaben (108). Laut einer dänischen Analyse von 2004 bis 2010 waren 11,7\% der Frauen zu Beginn der Schwangerschaft adipös (34).

Die Adipositas-Prävalenz in der Klinik St. Hedwig war im angegebenen Untersuchungszeitraum in etwa mit den von der BAQ ermittelten Werten für Gesamtbayern vergleichbar (siehe 1.1.2). Eine sächsische Studie, der 5067 Geburten der Universitätsklinik Leipzig von 2001 bis 2004 zugrunde lagen, ermittelte eine Adipositas-Häufigkeit von 18,8\%, was deutlich höher ist, verglichen mit den hier vorliegenden Auswertungen (64). Reiss et al. untersuchten die Daten von knapp 5300 Schwangeren im Zeitraum von zwölf Monaten 2011/2012, die in Berlin entbanden. Der Fokus lag dabei auf dem Einfluss eines Migrationshintergrundes der Frauen. Insgesamt betrug der Anteil adipöser Schwangerer 12,8\%, wobei bezogen auf die Nationalität 11,9\% der deutschen, 15,9\% der türkischen und 17,9\% der libanesischen Frauen zu Beginn der Schwangerschaft einen BMI $\geq 30 \mathrm{~kg} / \mathrm{m}^{2}$ aufwiesen (143). 


\subsection{Maternale Charakteristika}

In Übereinstimmung mit den Ergebnissen des BSG98 (8) war laut den aktuellen Daten der DEGS1-Studie eine Zunahme des mittleren BMI mit fortschreitendem Alter zu verzeichnen. Unter den 18 bis 29-jährigen Frauen lag der BMI im Mittel bei 23,7 kg/m² und stieg unter den 30 bis 39-jährigen bzw. 40 bis 49-jährigen Frauen auf 25,2 kg/m² bzw. 25,8 kg/m² an (9).

Dementsprechend war in der vorliegenden Studie in der Gruppe der übergewichtigen und adipösen Patientinnen ein höherer Mittelwert des maternalen Alters bei Entbindung gegenüber normalgewichtigen Frauen zu erwarten. Die Auswertungen ergaben hingegen keine deutlichen Altersunterschiede zwischen normalgewichtigen (31,2 Jahre), übergewichtigen (31,4 Jahre) und adipösen Patientinnen (31,2 Jahre). Als mögliche Begründung dafür ist ein Anstieg der Adipositas-Prävalenz insbesondere unter den jungen Frauen naheliegend. Die statistische Datenanalyse zeigte beim Vergleich der BMI-Kategorien Normalgewicht und Übergewicht ein signifikantes Ergebnis ( $\mathrm{p}=0,003$ ), was am ehesten durch die Größe der Stichprobe (N=27754) bedingt und als klinisch nicht relevant zu beurteilen ist.

Verschiedene Studien, deren Untersuchungszeiträume zwischen 2004 und 2012 lagen, bestätigten das Fehlen deutlicher Altersunterschiede zwischen den BMI-Gruppen (34, 36, 38). Den Resultaten einer dänischen Analyse zufolge (N=369347) schwankte der Mittelwert des mütterlichen Alters bei der Entbindung in den anhand des BMI eingeteilten Subgruppen zwischen 30,5 und 30,8 Jahren (34). Dagegen berichteten einige Studien, deren Beobachtungszeiträume in den 1980er- und 1990er-Jahren lagen, von einem Anstieg des mittleren Alters der Mütter mit zunehmender BMI-Kategorie (37, 61, 72, 106).

Ähnlich den Ergebnissen anderer Autoren (24, 29, 32, 34, 36, 37) hatten adipöse Schwangere in dieser Studie mit 58,4\% signifikant häufiger bereits ein oder mehrere Kinder geboren als normalgewichtige Frauen, unter denen der Anteil der Pluriparae bei 46,8\% lag.

15,0\% bzw. 5,1\% der Frauen, die im Untersuchungszeitraum in der Klinik St. Hedwig entbanden, waren in der Vorgeschichte von einem bzw. zwei oder mehr Aborten betroffen gewesen. In Bezug auf den mütterlichen BMI befanden sich adipöse Frauen mit 16,8\% bzw. 6,4\% signifikant häufiger im Z. n. einem bzw. zwei oder mehr Aborten in früheren Schwangerschaften, verglichen mit den Frauen des Referenzkollektivs mit 14,5\% bzw. 4,6\%. Diese Zusammenhänge bestätigten sich in der Subgruppenanalyse nach Ausschluss der Frauen mit Diabetes mellitus in der Schwangerschaft als statistisch signifikant. 
Auch in anderen Arbeiten wurde die maternale Adipositas als Risikofaktor für spontane und habituelle Aborte identifiziert (23-27). Laut den Ergebnissen einer 38 Studien umfassenden Metaanalyse aus dem Jahr 2014 stieg das Risiko einer Fehlgeburt ausgehend von normalgewichtigen Frauen pro Anstieg um fünf BMI-Einheiten der Mutter jeweils um das 1,2-fache an (26). In der Fall-Kontroll-Studie von Lashen et al. (N=4932) traten Frühaborte (6.-12. SSW) unter adipösen Frauen 1,2-mal wahrscheinlicher auf im Vergleich zu Schwangeren mit Normalgewicht. Auch das Risiko rezidivierender Fehlgeburten, definiert als vier oder mehr aufeinanderfolgende Frühaborte, zeigte sich in der Gruppe adipöser Frauen 3,5-fach erhöht gegenüber dem Referenzkollektiv (27). Es ist erwiesen, dass mütterliche Hyperglykämie bei der Konzeption mit einem erhöhten Abortrisiko einhergeht (144, 145). Auch nach Ausschluss der Frauen mit Diabetes mellitus kam es den Analysen Sheiners et al. zufolge (N=126080) jedoch bei adipösen Schwangeren (BMI $\geq 30 \mathrm{~kg} / \mathrm{m}^{2}$ ) mit 7,1\% signifikant häufiger zu zwei oder mehr aufeinanderfolgenden Aborten als bei nicht-adipösen Patientinnen (BMI $<30 \mathrm{~kg} / \mathrm{m}^{2}$ ) mit 4,8\% (25). Eine 16 Studien einschließende Metaanalyse ergab ein um den Faktor 1,7 erhöhtes Risiko für eine Fehlgeburt vor Vollendung der 20. SSW für Schwangere mit einem BMI $\geq 25 \mathrm{~kg} / \mathrm{m}^{2}$ gegenüber Frauen mit einem BMI $<25 \mathrm{~kg} / \mathrm{m}^{2}$, unabhängig davon, ob es sich um eine natürliche Konzeption oder einen reproduktiven Eingriff gehandelt hatte. Die Analyse in Subgruppen hinsichtlich der Konzeptionsart ergab, dass in Schwangerschaften, die mit Hilfe einer Eizellspende $(\mathrm{OR}=1,5)$ oder nach Ovulationsinduktion $(\mathrm{OR}=5,1)$ entstanden waren, ein hoher maternaler BMI mit einer gesteigerten Abortrate assoziiert war. Die Häufigkeit einer Fehlgeburt nach Invitro-Fertilisation bzw. Intrazytoplasmatischer Spermieninjektion zeigte dagegen keine Assoziation mit dem BMI der Schwangeren (23).

In Übereinstimmung mit den Beobachtungen anderer Autoren $(24,28,37,38,81)$ waren in dem dieser Studie zugrundeliegenden Kollektiv übergewichtige $(1,9 \%)$ und adipöse Frauen $(3,4 \%)$ signifikant häufiger bereits vor der Schwangerschaft an Diabetes mellitus erkrankt als Frauen mit Normalgewicht (0,6\%). Auch Fälle einer chronischen Hypertonie waren, entsprechend den Ergebnissen mehrerer Untersuchungen $(24,37,38,81,146)$, gegenüber der Referenzgruppe $(0,1 \%)$ unter den übergewichtigen $(0,2 \%)$ und adipösen Patientinnen $(1,1 \%)$ signifikant häufiger zu verzeichnen.

In der Klinik St. Hedwig nahmen übergewichtige und adipöse Frauen mit 13,6 kg bzw. 10,7 kg im Mittel in der Schwangerschaft signifikant weniger an Gewicht zu als normalgewichtige Frauen mit 14,3 kg. Auch die Ergebnisse der kanadischen Studie von Schummers et al. 
( $N=226958$ ) zeigten eine Abnahme der mittleren Gewichtszunahme der Schwangeren mit steigendem BMI. Normalgewichtige Frauen nahmen im Mittel 15,5 kg zu, übergewichtige Frauen 14,7 kg und Frauen mit Adipositas Grad I bis III 12,7 kg, 10,8 kg und 8,8 kg (36).

Trotzdem wurde die vom IOM empfohlene Gewichtszunahme in der Schwangerschaft in der hier vorliegenden Studie unter übergewichtigen mit 62,4\% und unter adipösen Frauen mit $59,1 \%$ signifikant häufiger überschritten als unter normalgewichtigen Patientinnen mit 36,1\%. Im Vergleich zur Referenzgruppe mit 34,4\% entsprach die Gewichtszunahme der übergewichtigen und adipösen Frauen mit 24,1\% bzw. 20,5\% signifikant seltener der Empfehlung des IOM. Eine Unterschreitung war unter normalgewichtigen Frauen mit 29,5\% signifikant häufiger zu beobachten als unter übergewichtigen und adipösen Frauen mit 13,5\% bzw. 20,4\%.

Auch den Ergebnissen einer US-amerikanischen Studie zufolge, die von 1995 bis 2002 durchgeführt wurde, überschritten übergewichtige mit 85,6\% und adipöse Patientinnen mit 78,7\% signifikant häufiger die angeratene Gewichtszunahme des IOM als normalgewichtigen Frauen mit 69,1\%. Der Anteil der Schwangeren mit exzessiver Gewichtszunahme war in jeder BMIKategorie im Vergleich zu den eigenen Arbeitsergebnissen höher, jedoch in Übereinstimmung damit in der BMI-Kategorie Übergewicht am höchsten (68).

Laut den Ergebnissen von Crane et al. entsprach die Gewichtszunahme in der Schwangerschaft bei insgesamt 30,6\% der Frauen dem empfohlenen Bereich des IOM. In der hier vorliegenden Studie lag der Anteil mit 30,7\% bei einem ähnlich niedrigen Wert. Dabei zeigten die Auswertungen von Crane et al., dass die Rate an Komplikationen (Kaiserschnitt, Gestationshypertonie, Geburtsgewicht $<2500$ g oder $\geq 4000 \mathrm{~g}$ ) in jeder BMI-Kategorie bei einer Einhaltung der für die Schwangerschaft empfohlenen Gewichtszunahme niedriger war als bei einer Überschreitung. Das geringste Risiko für Schwangerschaftskomplikationen wurde für Frauen mit einem präkonzeptionellen BMI zwischen $25 \mathrm{~kg} / \mathrm{m}^{2}$ und $40 \mathrm{~kg} / \mathrm{m}^{2}$ bei einer Gewichtszunahme von $6,7 \mathrm{~kg}$ bis $11,2 \mathrm{~kg}$ angegeben, während die Gewichtszunahme bei Frauen mit einem BMI $\geq 40 \mathrm{~kg} / \mathrm{m}^{2}$ unterhalb von $6,7 \mathrm{~kg}$ liegen sollte (147). Eine weitere große Kohortenstudie fand die niedrigste Komplikationsrate (Präeklampsie, Kaiserschnitt-Entbindung, SGA- und LGA-Kinder) in der BMI-Gruppe Adipositas Grad I mit einer Gewichtszunahme von 4,5 kg bis 11,3 kg, bei Frauen mit Adipositas Grad II mit einer Gewichtszunahme von $0 \mathrm{~kg}$ bis 4,1 $\mathrm{kg}$ und bei Patientinnen mit einem $\mathrm{BMI} \geq 40 \mathrm{~kg} / \mathrm{m}^{2}$ sogar bei einem Gewichtsverlust bis 4,1 kg (148).

Eine randomisiert-kontrollierte Studie zeigte zudem eine signifikante Verbesserung des Glukosemetabolismus bei einer angemessenen Gewichtszunahme im Schwangerschaftsverlauf bei 
adipösen Frauen. Während die Gewichtszunahme in einer Kontrollgruppe von Schwangeren (BMI $35 \pm 3 \mathrm{~kg} / \mathrm{m}^{2}$ ) im Mittel 13,3 $\mathrm{kg}$ betrug, gelang in der Interventionsgruppe (BMI 35 \pm 4 $\mathrm{kg} / \mathrm{m}^{2}$ ) mithilfe einer insgesamt zehnstündigen Ernährungsberatung eine Gewichtszunahme von lediglich 6,6 kg, was innerhalb des empfohlenen Bereichs des IOM liegt. In der 27. SSW waren in der Interventionsgruppe im Vergleich zur Kontrollgruppe sowohl die Nüchtern-Insulin als auch die Leptin-Werte um 20\% reduziert. Bei einer Messung in der 36. SSW war der Insulin-Wert in der Interventionsgruppe um 23\% und der Nüchtern-Blutglukose-Wert um 8\% niedriger als im Vergleichskollektiv (149).

Um maternale und neonatale Komplikationen zu vermindern, sollten insbesondere übergewichtige und adipöse Patientinnen über den Nutzen einer Einhaltung des vom IOM empfohlenen Bereichs der Gewichtszunahme in der Schwangerschaft informiert werden.

Die mittlere Aufenthaltsdauer in der Klinik St. Hedwig war bei übergewichtigen und adipösen Patientinnen mit 4,7 bzw. 5,1 Tagen signifikant länger als bei normalgewichtigen Frauen, die im Mittel 4,4 Tage in der Klinik blieben.

Auch den Befunden anderer Studien zufolge nahm die Länge des Krankenhausaufenthalts mit dem mütterlichen BMI zu $(32,37,64,70,150,151)$. Laut der US-amerikanischen Untersuchung von Chu et al. blieben übergewichtige und adipöse Frauen der Schweregrade I bis III mit 3,7, 4,0, 4,1 bzw. 4,4 Tagen im Mittel signifikant länger in der Klinik, verglichen mit 3,6 Tagen unter normalgewichtigen Patientinnen (150). Der Analyse Stepans et al. zufolge, der $5067 \mathrm{Ge}-$ burten der Universitätsklinik Leipzig im Zeitraum von 2001 bis 2004 zugrunde lagen, stieg die mittlere Aufenthaltsdauer ausgehend von 5,5 Tagen unter normalgewichtigen Frauen auf 6,7 Tage unter Frauen der BMI-Kategorie Adipositas Grad III an (64). In der prospektiven FallKontroll-Studie von Galtier-Dereure et al. war der verlängerte pränatale Krankenhausaufenthalt von Patientinnen mit einem BMI $>26,0 \mathrm{~kg} / \mathrm{m}^{2}$ gegenüber Schwangeren mit einem BMI von $18,0 \mathrm{~kg} / \mathrm{m}^{2}$ bis $25,0 \mathrm{~kg} / \mathrm{m}^{2}$ mit einer Erhöhung der medizinischen Versorgungskosten um das Fünffache assoziiert (151).

Laut dem aktuellen, sog. „Confidential Enquiry into Maternal Death 2016“ waren im Vereinigten Königreich im Zeitraum von 2012 bis 2014 insgesamt 200 mütterliche Todesfälle während der Schwangerschaft oder innerhalb von 42 Tagen nach der Entbindung zu verzeichnen. Davon standen insgesamt $51 \%$ der Fälle in direktem oder indirektem Zusammenhang mit mütterlichem Übergewicht (18\%) und Adipositas (33\%) in der Schwangerschaft (152). 


\subsection{Schwangerschaftsverlauf}

Maternale Adipositas gilt als etablierter Risikofaktor für die Entstehung eines GDM. In zahlreichen Studien zeichnete sich eine positive Assoziation zwischen einem hohen mütterlichen BMI und der GDM-Häufigkeit ab (24, 28-38). Im Vergleich zu normalgewichtigen Frauen deuteten die Ergebnisse der Metaanalyse von Chu et al. auf ein 2-fach, 4-fach und 9-fach erhöhtes GDM-Risiko für übergewichtige, adipöse und stark adipöse Schwangere hin (35). Eine dänische Kohortenstudie, der Daten von rund 370000 Geburten von 2004 bis 2010 zugrunde lagen, fand das Erkrankungsrisiko für übergewichtige Frauen 3-fach erhöht und für Frauen mit einem BMI zwischen $30,0 \mathrm{~kg} / \mathrm{m}^{2}$ und $34,9 \mathrm{~kg} / \mathrm{m}^{2}$ bzw. $\geq 35 \mathrm{~kg} / \mathrm{m}^{2}$ um den Faktor 8 bzw. 11 gesteigert, verglichen mit normalgewichtigen Schwangeren (34).

Auch in der vorliegenden Studie erkrankten übergewichtige $(9,0 \%)$ und adipöse Frauen $(18,5 \%)$ signifikant häufiger an GDM als normalgewichtige Frauen (5,3\%), wobei die Prävalenz mit jeder nächsthöheren BMI-Kategorie von Normalgewicht bis Adipositas Grad III $(28,2 \%)$ signifikant anstieg. Die GDM-Frequenz nahm mit dem BMI unabhängig von der mütterlichen Alterskategorie zu. Das Alter selbst spielt aber auch eine wichtige Rolle, die Prävalenz stieg mit dem Alter an und erreichte den höchsten Wert in der Altersgruppe über 35 Jahre in der BMI-Kategorie Adipositas Grad III mit 35,7\%.

Durch den kontinuierlichen Anstieg u. a. der Sexualsteroide, des humanen Plazentalaktogens und des Prolaktins während der Schwangerschaft entwickelt sich eine in der zweiten Hälfte der Gravidität zunehmende periphere Insulinresistenz im mütterlichen Organismus. Gesunde Schwangere können diese Insulinresistenz durch eine verstärkte Insulinfreisetzung ausgleichen und so eine normoglykäme Stoffwechsellage halten. Bei Gestationsdiabetikerinnen wird eine häufig bereits präkonzeptionell bestehende, chronische Herabsetzung der Insulinsensitivität durch die physiologisch einsetzende Insulinresistenz in der Schwangerschaft zusätzlich verstärkt, was bei gleichzeitig unzureichender $\beta$-Zell-Kompensation eine Hyperglykämie zur Folge hat. Auf der Basis einer genetischen Prädisposition wird die Ausprägung sowohl der Insulinresistenz als auch der Insulinsekretionsstörung wesentlich durch das Vorliegen von Adipositas sowie vom mütterlichen Lebensstil (ungesunde Ernährung, körperliche Inaktivität) beeinflusst. Neben den hormonellen Umstellungen in der Schwangerschaft scheint auch eine veränderte Freisetzung von Adipokinen (Adiponektin, Leptin) und Zytokinen (Tumornekrosefaktor $\alpha$, Interleukin-6) aus dem Fettgewebe und der Plazenta eine Rolle bei der Entstehung eines GDM zu spielen $(45,130)$. 
Laut den Daten der BAQ stieg die GDM-Prävalenz von 2000 bis 2015 von 0,8\% auf 4,3\% an (siehe Abb. 2). Auch war in Bayern eine Zunahme des Anteils Schwangerer über 35 Jahre von $18,8 \%$ auf 27,6\% sowie ein Anstieg der Rate adipöser Schwangerer von 10,0\% auf 12,5\% von 2000 bis 2015 zu beobachten (20). Screening und Diagnostik des GDM sind zudem seit März 2012 in Deutschland Bestandteil der gesetzlich verbindlichen Mutterschafts-Richtlinien (Mutterschafts-Richtlinien des Gemeinsamen Bundesausschusses). Der starke Anstieg der GDMPrävalenz um mehr als das Fünffache kann somit u. a. als Folge einer Zunahme der Prävalenz von Risikofaktoren sowie einer verbesserten Diagnostik angesehen werden.

Die Krankheitshäufigkeit des GDM lag in der hier vorliegenden Studie mit insgesamt 7,6\% bei höheren Werten im Vergleich zu den Angaben der BAQ mit 2,8\% in Gesamtbayern im Zeitraum von 2000 bis 2015 (20). Schwangere mit GDM sind Risikoschwangere. Ein Beschluss des Gemeinsamen Bundesausschusses im Jahr 2005 sieht die Entbindung von Schwangeren mit insulinpflichtigem Diabetes mellitus in einem Perinatalzentrum Level I oder II vor, um eine umfassende Primärversorgung des Kindes sicherzustellen (Qualitätssicherungs-Richtlinie Früh- und Reifgeborene (QFR-RL) des Gemeinsamen Bundesausschusses). Auch Patientinnen mit diätetisch eingestelltem GDM wird die Entbindung in einem Krankenhaus mit besonderer diabetologischer Erfahrung und neonatologischer Betreuung vor Ort laut den aktuellen Leitlinien angeraten (153). Als zertifiziertes Perinatalzentrum der höchsten Versorgungsstufe gewährleistet die Klinik St. Hedwig eine optimale Betreuung von Gestationsdiabetikerinnen und deren Neugeborener, weshalb betroffene Schwangere gehäuft St. Hedwig als Geburtsklinik wählen. Dies könnte den höheren Prozentsatz des GDM in dieser Studie im Vergleich zur allgemeinen Prävalenz in bayerischen Krankenhäusern erklären.

In Abhängigkeit der Werte der mehrmals täglich durchgeführten Blutglukosemessungen ist der GDM laut den aktuellen Leitlinien mit einer Ernährungs- und Bewegungstherapie oder zusätzlicher Insulintherapie zu behandeln. In Deutschland ist die Anwendung des Biguanidpräparates Metformin in der Schwangerschaft derzeit nicht zugelassen (153). Laut einer Publikation von Langer et al. benötigten adipöse Gestationsdiabetikerinnen häufiger eine Insulintherapie zur optimalen Blutzuckereinstellung als normalgewichtige Frauen mit GDM, um ein vergleichbares Schwangerschafts-Outcome zu erreichen (154).

In den randomisiert-kontrollierten Interventionsstudien von Landon et al. und Crowther et al. wurde untersucht, inwieweit sich eine spezifische Behandlung des GDM auf die Inzidenz peri- 
nataler Komplikationen auswirkte. In der Studie von Landon et al. zählten zu den Einschlusskriterien ein Blutglukose-Wert nüchtern $<95 \mathrm{mg} / \mathrm{dl}$ und eine Stunde nach einem $50 \mathrm{~g}$ oGTT 135-200 mg/dl. Bei Crowther et al. betrugen die Nüchternwerte der ausgewählten Studienteilnehmerinnen 73-99 mg/dl und die zwei-Stunden Werte nach einem 75g oGTT 140-198 mg/dl. Eine Kontrollgruppe von Frauen wurde routinemäßig betreut, während die Interventionsgruppe eine GDM-spezifische Therapie erhielt. In beiden Studien war in der Interventionsgruppe eine signifikante Reduktion der Inzidenz von Gestationshypertonie sowie von Geburtsgewichten über $4000 \mathrm{~g}$ oder über der 90. Perzentile zu beobachten. Zudem ergaben die Auswertungen von Landon et al., dass unter den spezifisch therapierten Frauen die Inzidenz einer KaiserschnittEntbindung, Schulterdystokie und Präeklampsie signifikant niedriger war, verglichen mit den routinemäßig behandelten Frauen $(155,156)$.

Neben den unter 1.1.3 vorgestellten, unmittelbaren Folgen für Mutter und Kind erhöht das Vorliegen eines GDM auch das Diabetesrisiko der Schwangeren im späteren Leben. 35\% bis 60\% der Frauen entwickeln nach einer Schwangerschaft, in der ein GDM auftrat, innerhalb von zehn Jahren eine Glukosetoleranzstörung $(44,45,153)$. Eine deutsche Studie untersuchte Frauen sechs Jahre nach einem aufgetretenen Schwangerschaftsdiabetes und fand bei 9,2\% der Frauen einen Typ II Diabetes mellitus und bei 19,1\% eine gestörte Glukosetoleranz (157). Sowohl für das Risiko einer Konversion eines GDM in einen manifesten Diabetes mellitus als auch für ein erneutes Auftreten der Glukosetoleranzstörung in Folgeschwangerschaften gilt die Adipositas der Mutter als etablierter Risikofaktor. Als weitere verstärkende Einflussfaktoren sind u. a. ein Gestationsalter kleiner 24 SSW bei Diagnosestellung und eine stattgefundene Insulintherapie während der Schwangerschaft zu nennen $(153,157)$. Bei den Nachkommen diabetischer Mütter ist durch die Perinatale Programmierung von lebenslang erhöhten diabetogenen, adipogenen und atherogenen Risiken auszugehen (133-135), wie es bereits in Kapitel 1.1.4 beschrieben wurde.

In Übereinstimmung mit den Beobachtungen verschiedener Autoren war in dem dieser Arbeit zugrundeliegenden Kollektiv eine positive Assoziation zwischen Adipositas in der Schwangerschaft und der Erkrankungsrate an Gestationshypertonie festzustellen (32, 36, 38, 40). Eine Präeklampsie trat in der Klinik St. Hedwig im Untersuchungszeitraum in 2,0\% der Schwangerschaften auf. Dieser Wert ist mit den Ergebnissen anderer Studien in etwa vergleichbar, in denen Prävalenzen zwischen 2,0\% und 5,0\% angegeben wurden $(28,36,38,158)$. Unabhängig 
von der Parität und dem Vorliegen einer diabetischen Stoffwechsellage erkrankten übergewichtige $(2,3 \%)$ und adipöse Frauen $(4,4 \%)$ in dieser Untersuchung signifikant häufiger an einer Präeklampsie als normalgewichtige Schwangere (1,5\%), wobei in der Subgruppe der Primiparae und der diabetischen Frauen jeweils eine höhere Präeklampsie-Rate zu verzeichnen war.

Die Resultate anderer Studien bestätigen diese Beobachtungen (24, 28, 32, 34, 36-40, 60). Im Vergleich zu Schwangeren mit Normalgewicht fanden Ovesen et al. ein 2-fach erhöhtes Präeklampsie-Risiko für übergewichtige Frauen, welches für adipöse Frauen mit einem BMI zwischen $30,0 \mathrm{~kg} / \mathrm{m}^{2}$ und $34,9 \mathrm{~kg} / \mathrm{m}^{2}$ bzw. $\geq 35,0 \mathrm{~kg} / \mathrm{m}^{2}$ auf das 3 -fache bzw. 4-fache anstieg (34). Eine kanadische Studie berichtete von einer nahezu linearen Assoziation zwischen dem steigenden mütterlichen BMI und der Präeklampsie-Rate ausgehend von normalgewichtigen Primiparae (36). Eine 29 Kohortenstudien umfassende Metaanalyse identifizierte mütterliche Adipositas als unabhängigen Risikofaktor für eine Präeklampsie. Auch nach Adjustierung für andere Einflussfaktoren war das relative Präeklampsie-Risiko für übergewichtige (BMI 25,0-29,9 $\mathrm{kg} / \mathrm{m}^{2}$ ), adipöse (BMI 30,0-34,9 kg/m²) und stark adipöse Frauen $\left(B M I \geq 35 \mathrm{~kg} / \mathrm{m}^{2}\right) \mathrm{im}$ Vergleich zu normalgewichtigen Schwangeren mit 1,7, 2,9 bzw. 4,1 signifikant erhöht (39). In der sog. „Hyperglycaemia and Adverse Pregnancy Outcome Study (HAPO)“ konnte der unabhängige Einfluss mütterlicher Adipositas von dem Blutglukosewert auf das Risiko einer Präeklampsie bestätigt werden (60). Maternale Fettleibigkeit war nach Angaben verschiedener Studien auch unabhängig von der Parität signifikant mit einem erhöhten Risiko für hypertensive Schwangerschaftserkrankungen assoziiert $(28,32,39)$, wobei die Erkrankungsrate laut Cnattingius et al. in der Gruppe der Primiparae mit 4,6\% höher war als unter Pluriparae mit 1,9\% (28).

In Übereinstimmung mit den Ergebnissen von Metsälä et al. (N=119485) war in dieser Studie kein statistisch signifikanter Zusammenhang zwischen dem Auftreten eines HELLP-Syndroms (157 Fälle) bzw. einer Eklampsie (16 Fälle) und dem mütterlichen BMI zu beobachten (38).

Die Ursachen der Präeklampsie sind noch nicht vollständig geklärt. Von entscheidender Bedeutung wird eine Plazentationsstörung mit ungenügender Invasion und Dilatation der Spiralarterien durch den extravillösen Trophoblasten angesehen. Durch die Hypoperfusion des intervillösen Raumes kommt es zu einer Ischämie des Trophoblasten und Verschiebungen bei vasoaktiven und angiogenetischen Faktoren. Daraus resultieren generalisierte Endothelveränderungen und eine systemische Inflammation, die u. a. eine Vasokonstriktion, Hyperpermeabilität und Thrombozytenaktivierung an den mütterlichen Gefäßen zur Folge haben (159). 
Wie bereits unter 1.1.4 beschrieben, ist Adipositas in der Schwangerschaft, auch bei ansonsten gesunden Müttern, mit Hypertonie, endothelialer Dysfunktion, chronischer Inflammation, Hyperinsulinämie und Dyslipidämie assoziiert. Obwohl Adipositas und Präeklampsie einige biochemische und physiologische Veränderungen gemeinsam haben, sind die Mechanismen, die dem Zusammenhang zwischen maternaler Adipositas und dem erhöhten Präeklampsie-Risiko zugrunde liegen, noch nicht klar $(39,40,130)$. Verschiedene Studien, die eine mögliche Rolle des C-reaktiven Proteins (160-162), der Matrix-Metalloproteasen (163), des Sexualhormonbindenden Globulins (164) sowie der Adipokine prüften $(165,166)$, konnten keinen eindeutigen kausalen Zusammenhang aufdecken.

In dieser Studie gaben übergewichtige $(1,8 \%)$ und adipöse Schwangere $(1,8 \%)$ signifikant häufiger an, während der Schwangerschaft geraucht zu haben als normalgewichtige Frauen (1,2\%). Auch andere Autoren berichteten von einer Zunahme der Nikotinabusus-Rate in der Schwangerschaft mit steigendem BMI (28, 32, 34, 36, 38). Während der Anteil der Raucherinnen in diesem Kollektiv von 1,2\% unter normalgewichtigen Frauen anstieg, wurden in anderen, hinsichtlich des Analysezeitraums vergleichbaren Studien deutlich höhere Ausgangswerte von 7,9\% bis 10,8\% angegeben $(34,36,38)$. Das Zigarettenrauchen während der Schwangerschaft gilt als protektiver Faktor gegenüber einer Präeklampsie-Erkrankung, kann aber zu einer Wachstumsrestriktion beim Feten führen (167).

Hinsichtlich des Vorliegens einer besonderen psychischen Belastung der Schwangeren $(\mathrm{n}=86)$ konnten keine signifikanten Unterschiede zwischen den BMI-Gruppen Normalgewicht $(0,3 \%)$, Übergewicht $(0,4 \%)$ und Adipositas $(0,2 \%)$ beobachtet werden. In der Klinik St. Hedwig wurden die Symptome einer beginnenden oder bereits bestehenden psychischen Erkrankung der Schwangeren nicht routinemäßig anhand eines Fragebogens o. ä. abgefragt, was zu einer Unterschätzung der tatsächlichen Häufigkeit geführt haben kann. Das muss bei der Interpretation der kleinen Zahl und des fehlenden Zusammenhangs zwischen Adipositas und einer psychischen Belastung der Schwangeren in dieser Untersuchung berücksichtigt werden.

Die Ergebnisse einer 62 Studien umfassenden Metaanalyse deuteten auf eine signifikante Assoziation des mütterlichen BMI vor der Schwangerschaft mit der Erkrankungsrate an Depressionen und Angststörungen hin. Das Depressions-Risiko war für übergewichtige und adipöse Frauen sowohl ante partum (1,2-fach bzw. 1,4-fach) als auch post partum (1,1-fach bzw. 1,3- 
fach) signifikant erhöht, verglichen mit normalgewichtigen Schwangeren. Auch das Risiko einer Angststörung während der Schwangerschaft war für adipöse Frauen im Vergleich zu Frauen mit Normalgewicht um den Faktor 1,4 gesteigert. Die mediane Prävalenz einer Depression lag für normalgewichtige, übergewichtige und adipöse Frauen während der Schwangerschaft bei $22,6 \%, 28,6 \%$ und 33,0\% sowie post partum bei 9,9\%, 11,8\% und 13,0\%. Zur Identifikation der Frauen mit Symptomen einer Depression wurden in den eingeschlossenen Studien spezielle Fragebögen wie z. B. der sog. „Edinburgh Postnatal Depression Scale“ (168) angewendet (41).

Hinsichtlich der Häufigkeit eines Harnwegsinfekts in der Schwangerschaft $(n=101)$ waren in dem dieser Studie zugrundeliegenden Kollektiv keine signifikanten Unterschiede zwischen den BMI-Kategorien Normalgewicht (0,3\%), Übergewicht $(0,5 \%)$ und Adipositas $(0,4 \%)$ festzustellen. Im Gegensatz dazu berichteten andere Autoren von einer signifikant höheren Rate an Harnwegsinfekten bei Schwangeren mit einem BMI $\geq 30 \mathrm{~kg} / \mathrm{m}^{2}$, verglichen mit normalgewichtigen Frauen $(24,37,42)$. Laut einer Studie, in der das Outcome von 287213 Schwangerschaften in London analysiert wurde, erkrankten übergewichtige $(0,8 \%)$ und adipöse Frauen $(1,1 \%)$, unabhängig des Vorliegens einer diabetischen Stoffwechsellage, signifikant häufiger an einem Harnwegsinfekt als normalgewichtige Patientinnen (0,7\%) (37).

In Bezug auf eine fetale intrauterine Wachstumsrestriktion $(n=798)$ wurde in dieser Untersuchung kein signifikanter Zusammenhang mit dem mütterlichen BMI beobachtet. Unter übergewichtigen und adipösen Frauen war diese mit 2,9\% bzw. 3,2\% prozentual etwas häufiger zu verzeichnen als unter den Schwangeren der Referenzgruppe mit 2,8\%. Auch den Resultaten anderer Studien zufolge war keine signifikant positive Assoziation der mütterlichen Adipositas mit der Häufigkeit eines IUGR festzustellen (72, 81, 146, 169). Im Gegenteil konnte mütterliche Adipositas als protektiver Faktor gegenüber einer Wachstumsrestriktion identifiziert werden, während mütterliches Untergewicht mit einer signifikant erhöhten IUGR-Rate in Verbindung gebracht wurde $(169,170)$. Eine rumänische Studie, die den Schwangerschaftsverlauf von 500 adipösen Frauen untersuchte, zeigte dagegen eine Zunahme der Inzidenz eines IUGR mit ansteigendem Schweregrad der Adipositas (Grad I: 14,4\%, Grad II: 26,7\%, Grad III: 50\%) (171). Die Aussagekraft dieser Studie ist jedoch durch das Fehlen einer normalgewichtigen Vergleichsgruppe sowie durch den kleinen Stichprobenumfang eingeschränkt. Die Zunahme der IUGR-Rate könnte laut Marchi et al. u. a. durch adipositas-assoziierte Komorbiditäten der Mutter erklärt werden, die mit ansteigendem Grad der mütterlichen Fettleibigkeit häufiger auftreten (172). 
Im Vergleich zu Frauen mit Normalgewicht (4,7\%), kam es in der Klinik St. Hedwig bei übergewichtigen $(3,5 \%)$ und adipösen Schwangeren $(3,1 \%)$ signifikant seltener zu vorzeitigen, zervixwirksamen Wehen. Die Ergebnisse von Konje et al. bestätigen diese Beobachtung (106).

\subsection{Geburtsverlauf}

Zwischen der Häufigkeit eines vorzeitigen Blasensprungs und der mütterlichen BMI-Kategorie war in dieser Studie keine signifikante Assoziation zu beobachten. Das Ereignis war bei übergewichtigen und adipösen Gebärenden mit 29,5\% bzw. 29,9\% prozentual etwas häufiger zu finden als in der Referenzgruppe mit 29,2\%. Auch andere Autoren berichteten über das Fehlen eines signifikanten Zusammenhangs zwischen dem mütterlichen BMI und dem Auftreten eines vorzeitigen Blasensprungs $(69,72,106)$.

In der Klinik St. Hedwig lag der Anteil der Kaiserschnitt-Entbindungen im Untersuchungszeitraum bei insgesamt 23,5\%. In Bezug auf den mütterlichen BMI waren in den Kategorien Übergewicht $(25,7 \%)$ und Adipositas (33,7\%) signifikant höhere Werte zu beobachten als in der Referenzgruppe $(21,1 \%)$. Der Anteil der primären und sekundären Sectiones lag unter übergewichtigen mit 12,8\% bzw. 12,9\% und adipösen Frauen mit 18,2\% bzw. 15,5\% bei signifikant höheren Werten im Vergleich zu normalgewichtigen Frauen mit 10,2\% bzw. 11,0\%. Der positive Zusammenhang zwischen mütterlicher Adipositas und der Rate primärer und sekundärer Kaiserschnitte zeigte sich durch Subgruppenanalysen als unabhängig von der Parität, dem Vorliegen einer Geburtseinleitung sowie von Komorbiditäten der Schwangeren.

In Übereinstimmung mit den vorliegenden Ergebnissen konnte maternale Adipositas in zahlreichen Untersuchungen als unabhängiger Risikofaktor für eine Entbindung per primärer oder sekundärer Sectio caesarea identifiziert werden $(24,25,29,32,34,36,37,42,57-68)$. Eine dänische Kohortenstudie $(\mathrm{N}=369347)$ berichtete von einem im Vergleich zu normalgewichtigen Frauen 1,2-fach, 1,5-fach bzw. 1,7-fach erhöhten Risiko für eine primäre Sectio caesarea für Frauen mit einem BMI von $25,0 \mathrm{~kg} / \mathrm{m}^{2}$ bis $29,9 \mathrm{~kg} / \mathrm{m}^{2}, 30,0 \mathrm{~kg} / \mathrm{m}^{2}$ bis $34,9 \mathrm{~kg} / \mathrm{m}^{2}$ und $\geq 35,0$ $\mathrm{kg} / \mathrm{m}^{2}$. Auch das Risiko, per sekundärer Sectio caesarea zu entbinden, war für die genannten BMI-Gruppen im Vergleich zur Referenzgruppe jeweils um den Faktor 1,3, 1,6 und 1,9 gesteigert (34). Eine 33 Studien umfassende Metaanalyse zeigte ein 1,5-fach, 2,1-fach bzw. 2,9-fach erhöhtes Kaiserschnitt-Risiko für übergewichtige, adipöse und stark adipöse Frauen verglichen mit normalgewichtigen Schwangeren (66). Laut einer US-amerikanische Studie war der SectioAnteil unter übergewichtigen und adipösen Frauen mit 30,7\% bzw. 39,2\% signifikant höher als 
unter normalgewichtigen Frauen mit 23,5\%, wobei eine allgemeine Kaiserschnitt-Rate von 29,2\% angegeben wurde (24). Eine australische Untersuchung berichtete von einem Anstieg der Sectio-Rate von 22,3\% unter normalgewichtigen Frauen auf 42,7\% unter Frauen mit einem BMI $>40,0 \mathrm{~kg} / \mathrm{m}^{2}$ (32). In einer kanadischen Studie betrug der Anteil der Schnittentbindungen innerhalb der untersuchten Primiparae 29,4\% (36). Im Vergleich dazu lag die Rate der abdominellen Entbindungen in der Klinik St. Hedwig mit insgesamt 23,5\% bzw. mit 25,6\% unter Primiparae bei deutlich niedrigeren Werten. Verschiedene Studien bestätigten, dass maternale Adipositas das Risiko für eine Sectio caesarea zusätzlich, aber unabhängig von einer diabetischen Stoffwechsellage der Schwangeren erhöht. Dabei wurde dem Einfluss des BMI auf das Kaiserschnitt-Risiko eine größere Bedeutung zugeschrieben als dem des GDM (57-60).

Zu den Gründen, die bei adipösen Frauen im Vergleich zur Referenzgruppe signifikant häufiger Anlass zu einem primären bzw. sekundären Kaiserschnitt gaben, zählten in dieser Studie der Z. n. Sectio, ein pathologisches oder suspektes CTG, ein pathologischer Dopplerbefund, der V.a. fetale Makrosomie, der Geburtsstillstand oder protrahierter Verlauf der EP und mütterliche Erkrankungen. Auch andere Autoren konnten die erhöhte Sectio-Rate bei adipösen Frauen auf die genannten Indikationen zurückführen $(25,37,67,68,76,81)$. Hinsichtlich der Sectio-Ursachen Plazenta praevia, vorzeitige Plazentalösung und Nabelschnurkomplikationen fanden andere Studien, analog zu den hier vorliegenden Auswertungen, keine signifikante Assoziation mit dem mütterlichen BMI $(25,37,67,72,146)$. Ein absolutes bzw. relatives Missverhältnis zwischen kindlichem Kopf und mütterlichem Becken, eine Wunschsectio und Lageanomalien gaben laut den Ergebnissen anderer Untersuchungen unter adipösen Frauen signifikant häufiger Anlass zu einem Kaiserschnitt als unter Frauen mit Normalgewicht (25, 37, 65). Diese Zusammenhänge konnten in der hier vorliegenden Analyse nicht bestätigt werden.

In dem dieser Studie zugrundeliegenden Kollektiv waren 338 (1,2\%) Not-Kaiserschnitte zu verzeichnen. Dabei bestand kein statistisch signifikanter Unterschied zwischen übergewichtigen $(1,3 \%)$ und adipösen Schwangeren (1,5\%) im Vergleich zu Normalgewichtigen $(1,2 \%)$. Andererseits wurde mütterliche Adipositas in einigen Studien mit einer signifikant erhöhten Frequenz von Not-Kaiserschnitten in Verbindung gebracht $(42,67,69,70)$. In der Untersuchung von Arrowsmith et al. kam es unter den 29224 analysierten Geburten mit 742 Fällen $(2,5 \%)$ häufiger zu einer Not-Sectio als in der hier vorliegenden Studie. Der Anteil stieg ausgehend von 2,4\% unter normalgewichtigen Schwangeren auf 4,1\% unter Frauen der BMI-Ka- 
tegorie Adipositas Grad III an (67). Eine 49 Studien umfassende Metaanalyse schätzte das Risiko einer Not-Sectio für fettleibige Frauen im Vergleich zu normalgewichtigen Frauen um den Faktor 1,6 erhöht (70).

Die Kinder adipöser Frauen wurden im Vergleich zu denen normalgewichtiger Frauen in der Klinik St. Hedwig signifikant seltener spontan vaginal aus Schädellage (61,2\% gegenüber $70,8 \%)$, vaginal-operativ (4,4\% gegenüber $6,9 \%)$ und in vaginaler Beckenendlage $(0,7 \%$ gegenüber 1,2\%) zur Welt gebracht. Mehrere Autoren bestätigten die signifikante Reduktion der spontanen vaginalen Geburten aus Schädellage sowie der vaginalen Beckenendlagen-Geburten unter adipösen Frauen $(32,37,42,68)$. Im Hinblick auf die Häufigkeit einer vaginal-operativen Entbindung bei adipösen Frauen im Vergleich zu Frauen mit Normalgewicht herrscht Uneinigkeit in der Literatur. Einige Autoren bestätigten ein erniedrigtes Risiko $(29,62,68)$, andere beschrieben ein erhöhtes Risiko für adipöse Frauen (70) und andere wiederum fanden keine signifikante Assoziation mit dem BMI der Mutter $(37,42,69)$.

Adipositas in der Schwangerschaft konnte in dieser Studie positiv mit der Frühgeburtlichkeit in Verbindung gebracht werden. Die Kinder adipöser Frauen wurden mit 15,1\% bzw. 2,9\% signifikant häufiger vor Vollendung der 37. SSW bzw. der 32. SSW zur Welt gebracht, als die Kinder normalgewichtiger Frauen mit 11,7\% bzw. 2,2\%. Eine Frühgeburt mit einem Gestationsalter kleiner 27+0 SSW trat im Untersuchungszeitraum in 171 Fällen auf (0,6\%). Dabei war der Anteil unter adipösen Frauen mit 0,7\% größer als unter normalgewichtigen Frauen mit 0,6\%, der Unterschied zwischen den Gruppen war jedoch nicht statistisch signifikant. In der Subgruppenanalyse, nach Ausschluss der Schwangeren mit einer hypertensiven Erkrankung oder diabetischen Stoffwechsellage, konnte die signifikante Assoziation zwischen mütterlicher Fettleibigkeit und dem gehäuften Auftreten von Entbindungen vor Vollendung der 37. SSW und der 32. SSW bestätigt werden. Die Rate der Entbindungen mit einem Schwangerschaftsalter kleiner 27+0 SSW war in dieser Subgruppe bei adipösen Frauen $(1,0 \%)$ signifikant höher als bei normalgewichtigen Frauen $(0,6 \%)$.

Hinsichtlich der Assoziation zwischen Adipositas in der Schwangerschaft und dem Risiko einer Frühgeburt wird in der Literatur nicht einheitlich berichtet, was an unterschiedlichen Klassifikationen des Gestationsalters und des maternalen BMI sowie an verschiedenen Ein- und Ausschlusskriterien der Studien liegen könnte. Zahlreiche Autoren bestätigten eine signifikante Assoziation zwischen maternaler Adipositas und einer gesteigerten Rate an Frühgeburten $(28,29$, 32, 36, 70-75), während anderen Studien zufolge das Risiko einer Frühgeburt mit steigendem 
mütterlichen BMI abnahm $(37,146)$. Laut den Ergebnissen anderer Analysen wiederum war kein signifikanter Zusammenhang zwischen dem maternalen BMI und dem Gestationsalter festzustellen $(25,64,81)$.

In der prospektiven Kohortenstudie von Magann et al. kam es bei adipösen Frauen mit 18,6\% signifikant häufiger zu einer Frühgeburt als bei normalgewichtigen Frauen mit 14,2\% (71). Auch Schummers et al. berichteten von einem signifikanten Anstieg der Entbindungen vor Vollendung der 37. SSW mit zunehmendem mütterlichen BMI von 7,1\% unter normalgewichtigen Frauen auf 10,3\% unter Frauen mit Adipositas Grad III (36). Das Risiko für eine Entbindung vor Vollendung der 33. SSW war laut Raja et al. für übergewichtige, adipöse (BMI 30,0$40,0 \mathrm{~kg} / \mathrm{m}^{2}$ ) und stark adipöse Frauen (BMI $>40 \mathrm{~kg} / \mathrm{m}^{2}$ ) im Vergleich zu Frauen mit Normalgewicht um den Faktor 1,4, 1,5 bzw. 1,2 erhöht (29). Nach einer schwedischen Kohortenstudie, der 610969 Einlingsgeburten zugrunde lagen, stieg das Risiko sowohl für eine Frühgeburt vor Vollendung der 37. SSW als auch der 32. SSW mit anwachsendem BMI kontinuierlich und statistisch signifikant an. Der Anteil der Geburten mit einem Schwangerschaftsalter $<37+0$ SSW bzw. $<32+0$ SSW nahm ausgehend von $4,5 \%$ bzw. $0,6 \%$ unter normalgewichtigen Frauen auf 7,7\% bzw. 1,3\% unter stark adipösen Frauen (BMI $\left.>40 \mathrm{~kg} / \mathrm{m}^{2}\right) \mathrm{zu}(74)$. Hinsichtlich einer Frühgeburt mit einem Schwangerschaftsalter kleiner 28+0 SSW beschrieb eine finnische Studie ein 1,5-fach erhöhtes Risiko für Frauen mit einem BMI $\geq 30 \mathrm{~kg} / \mathrm{m}^{2}$ gegenüber normalgewichtigen Schwangeren (73). Diese Beobachtungen stützen die eigenen Ergebnisse.

Cnattingius et al. untersuchten in ihrer groß angelegten Studie $(\mathrm{N}=1599551)$ den Zusammenhang zwischen dem maternalen BMI und der Häufigkeit einer frühen (32+0-36+6 SSW), sehr frühen (28+0-31+6 SSW) und extrem frühen (22+0-27+6 SSW) Frühgeburt. Dabei wurde zwischen medizinisch-indizierten Frühgeburten (Geburtseinleitung, primärer Kaiserschnitt) und spontanen Frühgeburten (vorzeitige Wehen, vorzeitiger Blasensprung) unterschieden. Das Risiko einer frühen, sehr frühen sowie extrem frühen medizinisch-indizierten Frühgeburt stieg dabei mit zunehmendem mütterlichen BMI unter übergewichtigen und adipösen Schwangeren im Vergleich zu Frauen mit Normalgewicht statistisch signifikant an (75). Dies wurde als Folge von adipositas-assoziierten Komorbiditäten angesehen. Ein unzureichend einstellbarer Diabetes mellitus in der Schwangerschaft bzw. das Vorliegen einer schweren Präeklampsie stellen u. a. Indikationen für eine vorzeitige Beendigung der Schwangerschaft dar (47, 54). Hinsichtlich der Häufigkeit einer frühen bzw. sehr frühen spontanen Frühgeburt war keine signifikante Assoziation mit dem mütterlichen BMI zu beobachten. Das Risiko für eine spontane, extrem frühe 
Frühgeburt war jedoch für adipöse Frauen im Vergleich zu normalgewichtigen Frauen signifikant erhöht und stieg mit zunehmendem Grad der mütterlichen Fettleibigkeit an (Grad I: aOR=1,2; Grad II: aOR=1,6; Grad III: aOR=2,1). Diese Beobachtung blieb auch nach Ausschluss der Frauen mit einer hypertensiven Erkrankung oder diabetischen Stoffwechsellage in der Schwangerschaft signifikant bestehen. Laut den Autoren könnte die chronische Inflammation, die nach Ramsay et al. auch bei ansonsten gesunden Frauen mit Adipositas in der Schwangerschaft einhergeht (130), durch das Auslösen von Zervixreifung und Myometriumkontraktionen eine Rolle in dem zugrundeliegenden Pathomechanismus spielen (75).

In Übereinstimmung mit den Ergebnissen anderer Publikationen $(37,67,70,74)$ trat in dem dieser Studie zugrundeliegenden Kollektiv die zeitliche Übertragung der Schwangerschaft ( $\geq 42+0$ SSW) unter übergewichtigen und adipösen Müttern mit 2,6\% bzw. 2,7\% signifikant häufiger auf als im Referenzkollektiv normalgewichtiger Schwangerer mit 2,1\%.

Adipöse Frauen haben aufgrund endokrinologischer Veränderungen häufig einen unregelmäBigen Menstruationszyklus, was die Berechnung des voraussichtlichen Geburtstermins anhand der Angaben zur letzten Regel erschwert. Eine exakte Bestimmung des Gestationsalters sollte daher laut Schäfer-Graf et al. bei adipösen Patientinnen über wiederholte Messungen der fetalen Scheitel-Steiß-Länge im ersten Trimenon erfolgen (173).

Zwischen dem mütterlichen BMI und der Häufigkeit einer Geburtseinleitung war in dieser Studie eine positive Assoziation festzustellen, wobei insgesamt 23,1\% der Geburten eingeleitet wurden. Bei übergewichtigen und adipösen Schwangeren wurde die Geburt mit einem Anteil von $25,8 \%$ bzw. $33,7 \%$ signifikant häufiger eingeleitet als bei normalgewichtigen Frauen mit 20,4\%. Der Zusammenhang erwies sich als unabhängig von der Parität und dem Vorliegen einer diabetischen Stoffwechsellage oder hypertensiven Erkrankung der Mutter. Zu den Gründen, die unter adipösen Frauen signifikant häufiger Anlass zu einer Geburtseinleitung gaben als unter normalgewichtigen Frauen, zählten mütterlicher Diabetes mellitus (10,6\% gegenüber 2,0\%), V. a. Makrosomie (7,8\% gegenüber 2,9\%), mütterliche Erkrankung (7,2\% gegenüber $3,7 \%)$ und Präeklampsie (7,1\% gegenüber 3,8\%).

In Übereinstimmung mit den hier vorliegenden Ergebnissen fanden zahlreiche Autoren eine signifikante, von anderen Risikofaktoren unabhängige Assoziation zwischen Übergewicht bzw. Adipositas in der Schwangerschaft und einer erhöhten Rate an Geburtseinleitungen. Dabei schwankte der Anteil der eingeleiteten Geburten in den zitierten Studien insgesamt zwischen 
$18,2 \%$ und $29,1 \%(24,25,29,37,42,67,68,76)$. Sebire et al. beschrieben ein 1,3-fach bzw. 1,7-fach erhöhtes Risiko für eine Geburtseinleitung für übergewichtige und adipöse Schwangere im Vergleich zu Frauen mit Normalgewicht (37). Laut Raja et al. wurde die Geburt von übergewichtigen, adipösen $\left(30,0-40,0 \mathrm{~kg} / \mathrm{m}^{2}\right)$ und stark adipösen Schwangeren (BMI $\geq 40,0$ $\mathrm{kg} / \mathrm{m}^{2}$ ) 1,4-mal, 1,6-mal bzw. 2,4-mal häufiger eingeleitet, verglichen mit normalgewichtigen Frauen (29).

Ein Wehenmittel in Form eines Oxytocin-Tropfs wurde bei der Geburt von 41,6\% der Frauen eingesetzt, wobei die Entbindungen per primärer Sectio caesarea von der Analyse ausgeschlossen waren (N=24518). Im Vergleich zur Referenzgruppe mit 40,8\%, war der Anteil der Entbindungen, bei denen die Wehen mithilfe eines Oxytocin-Tropfs unterstützt wurden, unter übergewichtigen und adipösen Frauen mit 42,2\% bzw. 45,2\% signifikant größer.

Auch den Analysen anderer Autoren zufolge bestand eine signifikante Assoziation zwischen maternaler Adipositas und dem gehäuften Einsatz eines Wehenmittels unter der Geburt $(62,68$, 70), wohingegen Raja et al. keinen signifikanten Zusammenhang fanden (29). Laut Hollowell et al. war die Rate der Oxytocin-Unterstützungen unter übergewichtigen, adipösen (BMI 30,0$\left.35,0 \mathrm{~kg} / \mathrm{m}^{2}\right)$ und stark adipösen Gebärenden (BMI >35,0 kg/m²) mit 24,6\%, 27,3\% bzw. 27,2\% signifikant größer als unter normalgewichtigen Frauen mit 23,2\%. Dabei waren Frauen mit adipositas-assoziierten Komorbiditäten, einer Entbindung per primärer Sectio caesarea und einem Gestationsalter kleiner 37 SSW von der Analyse ausgeschlossen (62). Nach Angaben einer USamerikanische Studie wurde bei übergewichtigen $(68,7 \%)$ und adipösen Primiparae $(75,0 \%)$ signifikant häufiger ein Wehenmittel unter der Geburt eingesetzt als bei normalgewichtigen Primiparae $(56,2 \%)(68)$.

Im Untersuchungszeitraum wurden 91 Fälle $(0,4 \%)$ einer Schulterdystokie verzeichnet. Der Anteil der Frauen, bei denen es unter der Geburt zu einer Schulterdystokie kam, war unter übergewichtigen und adipösen Gebärenden mit jeweils 0,7\% signifikant größer als unter normalgewichtigen Frauen mit 0,3\%. Dieser Zusammenhang zeigte sich als unabhängig von einer diabetischen Stoffwechsellage der Mutter, einer vaginal-operativen Entbindung und der Geburt eines als LGA eingestuften Kindes. In der Subgruppenanalyse bzgl. der Parität der Frau konnte der Zusammenhang zwischen maternaler Adipositas und der erhöhten Schulterdystokie-Rate unter Pluriparae als signifikant bestätigt werden, in der Gruppe der Primiparae jedoch nicht, was am ehesten auf die niedrige Fallzahl $(n=27)$ in dieser Subgruppe zurückzuführen ist. 
Verschiedene Autoren bestätigten eine im Vergleich zu normalgewichtigen Frauen signifikante Häufung der Schulterdystokie-Fälle bei adipösen Gebärenden, wobei der statistisch signifikante Zusammenhang auch nach Adjustierung für die Parität, das Vorliegen eines Diabetes mellitus und das kindliche Geburtsgewicht bestehen blieb (24, 36, 42, 64). Der Anteil der Geburten mit Auftreten einer Schulterdystokie stieg laut einer kanadischen Studie (N=226958) ausgehend von 3,5\% unter normalgewichtigen Frauen auf 4,1\% in der BMI-Kategorie Adipositas Grad III an, wobei die Schulterdystokie-Rate insgesamt bei 3,6\% lag (36). In einer US-amerikanischen Studie betrug die Inzidenz der Schulterdystokie 2,4\% (24), während eine dänische Studie eine Häufigkeit von 1,0\% berichtete (34). Im Vergleich zu diesen Prozentangaben trat die Schulterdystokie in der Klinik St. Hedwig mit einem Anteil von 0,4\% deutlich seltener auf.

In anderen Studien wiederum war kein signifikanter Zusammenhang zwischen dem mütterlichen BMI und der Inzidenz der Schulterdystokie festzustellen $(25,67,81,146)$.

Ovesen et al. ( $\mathrm{N}=369347)$ fanden in der unadjustierten Analyse ein um den Faktor 1,3, 1,4 bzw. 2,0 erhöhtes Schulterdystokie-Risiko für Gebärende mit einem BMI von $25,0 \mathrm{~kg} / \mathrm{m}^{2}$ bis 29,9 $\mathrm{kg} / \mathrm{m}^{2}, 30,0 \mathrm{~kg} / \mathrm{m}^{2}$ bis $34,9 \mathrm{~kg} / \mathrm{m}^{2}$ bzw. $\geq 35,0 \mathrm{~kg} / \mathrm{m}^{2}$ im Vergleich zu Frauen der BMI-Kategorie Normalgewicht. Dieser signifikante Zusammenhang war jedoch nach Adjustierung für mütterliches Alter, Parität, GDM, Nikotinabusus, Gestationsalter und Geburtsgewicht nicht mehr zu beobachten (34). Auch laut der Studie von Robinson et al. war maternale Adipositas nicht als unabhängiger Risikofaktor für eine Schulterdystokie anzusehen. Dem Einfluss fetaler Makrosomie mit einem Geburtsgewicht zwischen $4000 \mathrm{~g}$ und $4499 \mathrm{~g}(\mathrm{aOR}=9,0) \mathrm{bzw}$. >4500 g $(\mathrm{aOR}=39,5)$ wurde dagegen die größte, von anderen Risikofaktoren unabhängige Bedeutung bzgl. des Schulterdystokie-Risikos zugeschrieben (80).

$\mathrm{Zu}$ einer kindlichen Geburtsverletzung infolge einer Schulterdystokie kam es in der Klinik St. Hedwig von 2000 bis 2015 in vier Fällen. Aufgrund der geringen Fallzahl war die statistische Auswertung bzgl. einer etwaigen Assoziation mit dem mütterlichen BMI nicht möglich. Bei der Geburt von drei normalgewichtigen Frauen traten zwei Fälle einer Verletzung des Plexus brachialis und ein Fall einer Klavikulafraktur auf. Bei der Entbindung einer übergewichtigen Frau kam es zu einer kindlichen Erb-Lähmung.

In einer groß angelegten, kanadischen Kohortenstudie ( $\mathrm{N}=226958)$ waren 8231 Fälle einer Schulterdystokie und 187 Fälle einer kindlichen Verletzung infolgedessen zu verzeichnen $(0,1 \%)$. Dabei konnte gezeigt werden, dass die Kinder adipöser Frauen signifikant häufiger 
von einer Verletzung infolge einer Schulterdystokie betroffen waren als die Kinder normalgewichtiger Frauen (36). Auch Mehta et al. stellten eine signifikante Assoziation zwischen maternaler Fettleibigkeit und einem erhöhten kindlichen Verletzungsrisiko durch eine Schulterdystokie fest. Die Autoren begründeten dies u. a. mit der erschwerten Durchführung von Geburtsmanövern zur Lösung der Schulterdystokie bei adipösen Patientinnen. Zudem konnten ein kindliches Geburtsgewicht über $4500 \mathrm{~g}$ und eine Austreibungsphase länger als 20 Minuten als Risikofaktoren für eine kindliche Verletzung auf Grund einer Schulterdystokie identifiziert werden (77).

Grünes Fruchtwasser wurde in der hier untersuchten Stichprobe Gebärender bei übergewichtigen $(9,9 \%)$ und adipösen Patientinnen (11,3\%) im Vergleich zur Referenzgruppe $(8,3 \%)$ signifikant häufiger beobachtet. Die vorzeitige Mekoniumausscheidung des Fetus ist auf die peristaltikanregende Wirkung einer Kohlenstoffdioxid-Vermehrung im Blut zurückzuführen und gilt als Hinweis auf eine vorausgegangene fetale Hypoxie. Ein positiver Zusammenhang zwischen dem maternalen BMI und der Häufigkeit von Mekonium im Fruchtwasser konnte auch in anderen Studien festgestellt werden $(25,62,70,81,82)$. Laut Bianco et al. war der Anteil von grünem Fruchtwasser unter adipösen Gebärenden (BMI >35,0 kg/m²) mit 17,2\% signifikant höher, verglichen mit 13,0\% unter Frauen mit einem BMI von $19,0 \mathrm{~kg} / \mathrm{m}^{2}$ bis $27,0 \mathrm{~kg} / \mathrm{m}^{2}$ $(81)$.

Die Dauer der vaginalen Entbindung aus Schädellage war sowohl unter Primiparae als auch unter Pluriparae bei adipösen Gebärenden mit 9,1 bzw. 5,9 Stunden im Mittel länger als bei normalgewichtigen Frauen mit 8,7 bzw. 5,4 Stunden. Ein protrahierter Verlauf bzw. ein Geburtsstillstand in der EP wurde unter adipösen Patientinnen mit 1,8\% bzw. 5,0\% häufiger verzeichnet als im Referenzkollektiv mit 1,4\% bzw. 2,6\%. Dagegen war bei der Entbindung adipöser Frauen mit 1,8\% bzw. 4,2\% seltener ein protrahierter Verlauf bzw. Stillstand in der AP festzustellen als unter normalgewichtigen Frauen mit 3,0\% bzw. 4,4\%.

Zahlreiche Studien bestätigten die Beobachtung einer verlängerten EP und verkürzten AP bei der Geburt adipöser Frauen im Vergleich zu der von Frauen mit Normalgewicht $(25,42,67$, $68,76)$.

In einer israelischen Studie ( $\mathrm{N}=126080)$ trat der Geburtsstillstand in der EP bei adipösen Gebärenden (BMI $\left.\geq 30 \mathrm{~kg} / \mathrm{m}^{2}\right)$ mit 6,0\% signifikant häufiger auf als bei nicht-adipösen Frauen $\left(\mathrm{BMI}<30 \mathrm{~kg} / \mathrm{m}^{2}\right)$ mit 1,6\%, wobei Schwangere mit hypertensiven Erkrankungen oder Diabetes 
mellitus vom untersuchten Studienkollektiv ausgeschlossen waren (25). Laut Vahratian et al. war die mediane Dauer der Zervixerweiterung von $4 \mathrm{~cm}$ auf $10 \mathrm{~cm}$ bei adipösen Primiparae mit 7,9 Stunden signifikant länger als bei normalgewichtigen Primiparae mit 6,2 Stunden. Als Erklärungsgrund dafür zogen die Autoren in Erwägung, dass das Oxytocin selbst oder seine Rezeptoren von einem hohen mütterlichen BMI beeinflusst werden (68). In einer Studie, die den Zusammenhang zwischen maternaler Adipositas und der Kontraktilität der Uterusmuskulatur untersuchte, konnte gezeigt werden, dass das Myometrium adipöser Frauen im Vergleich zu dem normalgewichtiger Frauen mit geringerer Kraft und Frequenz kontrahiert. Das intrazelluläre, freie Kalzium, welches eine Voraussetzung für die Kontraktion der glatten Muskelzellen darstellt, war in der Gruppe der adipösen Frauen vermindert (76). Adipositas in der Schwangerschaft steht, auch bei ansonsten gesunden Frauen, mit einer im Vergleich zu normalgewichtigen Müttern erhöhten Konzentration der Nüchtern-Triglyzeride und des Low Density Lipoproteins (LDL) sowie einer erniedrigten Konzentration des High Density Lipoproteins (HDL) in Zusammenhang (130). In Tierversuchen konnte demonstriert werden, dass Cholesterin, ein wichtiger Bestandteil von Zellmembranen, Einfluss auf die Kontraktion der glatten Muskelfasern des Uterus im Sinne einer Verminderung der Kontraktilität nimmt (174). Insbesondere die Effektivität von Östrogen- und Oxytocin-Rezeptoren im Myometrium scheint vom Gehalt des Cholesterins in der Zellmembran moduliert zu werden (175). Die mit maternaler Adipositas einhergehende Hypercholesterinämie könnte demnach als ursächlich für eine Verminderung der Uterus-Aktivität und eine längere Dauer der EP bei adipösen Frauen im Vergleich zu normalgewichtigen Frauen angesehen werden (76).

Arrowsmith et al. berichteten, dass die mediane Dauer der AP bei der Geburt von übergewichtigen und adipösen Frauen mit 0,6 bzw. 0,5 Stunden signifikant kürzer war als unter Frauen der BMI-Kategorie Normalgewicht mit 0,9 Stunden (67). Eine andere Studie zeigte eine signifikant kürzere Dauer der AP bei fettleibigen Erstgebärenden (47,4 Minuten) verglichen mit normalgewichtigen Erstgebärenden (62,7 Minuten) (68). Den Ergebnissen Sheiners et al. zufolge trat der Geburtsstillstand in der AP unter Gebärenden mit einem BMI $\geq 30 \mathrm{~kg} / \mathrm{m}^{2}$ mit 1,5\% seltener auf als unter Frauen mit einem BMI $<30 \mathrm{~kg} / \mathrm{m}^{2}$ mit $1,7 \%$ (25).

Peripartale Blutungen stellen eine geburtshilfliche Notfallsituation dar. Sie sind mit ca. 13\% der maternalen Todesfälle in den Industrieländern sowie mit über 30\% der Müttersterblichkeit in der Dritten Welt assoziiert (55). 
Übergewichtige und adipöse Patientinnen verloren in der vorliegenden Studie im Mittel mit 405,4 ml bzw. 426,6 ml signifikant mehr Blut unter der Geburt als normalgewichtige Frauen mit 398,9 ml. Hinsichtlich der Häufigkeit einer PPH waren allerdings keine signifikanten Unterschiede zwischen den BMI-Gruppen festzustellen. Ein Blutverlust $\geq 500 \mathrm{ml}$ bei vaginaler Entbindung war unter adipösen Frauen mit 7,4\% prozentual häufiger zu beobachten als unter normalgewichtigen Müttern mit 6,8\%. Ein Blutverlust $\geq 1000 \mathrm{ml}$ bei Kaiserschnitt-Entbindung trat unter fettleibigen Frauen dagegen mit 0,8\% seltener auf als im Referenzkollektiv mit 1,6\%. Auch anderen Studien zufolge konnte kein statistisch signifikanter Unterschied bzgl. der Inzidenz peripartaler Blutungen zwischen dem adipösen und normalgewichtigen Kollektiv gefunden werden $(25,29,34,36,67)$. Laut der Analyse von Ovesen et al. schwankte die Häufigkeit einer PPH >500 $\mathrm{ml}$ in Bezug auf die maternalen BMI-Kategorien zwischen 6,4\% und 6,7\% (34). Arrowsmith et al. berichteten von einer Inzidenz der PPH $>500 \mathrm{ml}$ bei vaginaler Entbindung von $17,1 \%, 16,0 \%$ bzw. $15,1 \%$ unter normalgewichtigen, übergewichtigen und adipösen Gebärenden. Ein Blutverlust $>1000 \mathrm{ml}$ bei abdominellem Entbindungsmodus trat unter normalgewichtigen, übergewichtigen und adipösen Patientinnen in 8,0\%, 10,0\% bzw. 10,3\% der Fälle auf (67). In einer weiteren, groß angelegten Studie ( $=226958)$ war die PPH definiert als eine Blutung, die einer Intervention zur Blutstillung bedurfte (u. a. Bluttransfusion, Embolisation bzw. Ligation von Gefäßen, Hysterektomie). Eine signifikante Assoziation mit dem maternalen BMI war nicht festzustellen, wobei der Anteil der Hämorrhagien bei insgesamt 0,7\% lag (36). Im Unterschied zu den eigenen Arbeitsergebnissen fanden andere Autoren eine signifikante Assoziation zwischen dem mütterlichen BMI und der Häufigkeit einer PPH (24, 37, 42, 70, 76, 83). Laut der Untersuchung von Sebire et al. war ein mütterlicher Blutverlust $>1000 \mathrm{ml}$ bei übergewichtigen $(1,8 \%)$ und adipösen Gebärenden $(2,5 \%)$ signifikant häufiger zu beobachten, als im normalgewichtigen Vergleichskollektiv (1,4\%). Die Autoren nannten als möglichen Erklärungsgrund eine stärkere Blutung von der mit einem LGA-Kind assoziierten, relativ vergröBerten Implantationsfläche der Plazenta (37). Auch nach Ausschluss der Frauen mit einer hypertensiven Erkrankung oder diabetischen Stoffwechsellage in der Schwangerschaft fanden Kiran et al. die Rate der PPH >500 ml bei adipösen Primiparae mit 22,2\% signifikant erhöht gegenüber nicht-adipösen Primiparae (BMI 20,0-30,0 kg/m²) mit 15,5\% (42). Den Analysen Blombergs zufolge stieg die Inzidenz der postpartalen Blutung >1000 ml aufgrund einer Uterusatonie mit zunehmendem mütterlichen BMI signifikant an. Während unter normalgewichtigen Frauen 1,6\% betroffen waren, stieg der Anteil auf 1,8\% unter Frauen mit Adipositas Grad 
III an (83). Auch Zhang et al. führten die positive Assoziation zwischen maternaler Adipositas und einer PPH auf das gehäufte Auftreten einer uterinen Atonie zurück, die mit einer im Vergleich zu normalgewichtigen Frauen verminderten Kontraktilität der Uterusmuskulatur bei adipösen Frauen begründet wurde (76).

Da zwischen den verschiedenen Studien Unterschiede hinsichtlich der Messmethode des mütterlichen Blutverlustes sowie der Definition einer Peripartalen Hämorrhagie bestehen, ist die Vergleichbarkeit der Ergebnisse zum Teil eingeschränkt. 


\subsection{Kindliches Outcome}

In Übereinstimmung mit den Befunden anderer Studien (24, 29, 32, 34, 36, 68, 70, 84), waren die Neugeborenen übergewichtiger und adipöser Mütter in der Klinik St. Hedwig im Mittel mit 3348,3 g bzw. 3338,7 g signifikant schwerer als die Kinder normalgewichtiger Patientinnen mit 3260,2 g. Einige Autoren berichteten von einem kontinuierlichen Anstieg des kindlichen Geburtsgewichts mit zunehmendem maternalen BMI $(29,32,34,36)$. In einer kanadischen Studie stieg das mittlere Geburtsgewicht ausgehend von $3391 \mathrm{~g}$ unter den Neugeborenen normalgewichtiger Frauen auf $3619 \mathrm{~g}$ unter den Kindern von Frauen mit einem BMI $>40$ kg/m² konstant an (36). Andere Autoren fanden dagegen, analog zu den eigenen Auswertungen, das Geburtsgewicht der Neugeboren übergewichtiger Frauen im Mittel am höchsten (24, 68). Um die Ursache des erhöhten Körpergewichts der Kinder adipöser Frauen gegenüber normalgewichtiger Frauen zu prüfen, wurden in der Studie von Sewell et al. Neugeborene von glukosetoleranten Frauen untersucht. Dabei war der prozentuale Körperfett-Anteil der Neugeborenen von Frauen mit einem $B M I \geq 25 \mathrm{~kg} / \mathrm{m}^{2}$ mit $11,6 \%$ signifikant höher als bei Frauen mit einem BMI $<25$ $\mathrm{kg} / \mathrm{m}^{2}$ mit 9,7\%, während bzgl. des Anteils der fettfreien Körpermasse keine signifikanten Unterschiede zwischen den Gruppen zu finden waren (84).

Die Rate der als LGA klassifizierten Neugeborenen war in St. Hedwig unter übergewichtigen und adipösen Patientinnen mit 13,1\% bzw. 16,5\% signifikant höher als im Referenzkollektiv mit 7,8\%. Die Assoziation bestätigte sich durch Subgruppenanalyse als unabhängig von einer diabetischen Stoffwechsellage der Schwangeren, wobei der Anteil der LGA-Kinder von 9,9\% im Gesamtkollektiv auf 9,5\% in der Subgruppe stoffwechselgesunder Frauen sank.

Auch andere Studien berichteten von einer signifikanten, positiven Korrelation zwischen dem maternalen BMI und der Häufigkeit eines als LGA eingestuften Neugeborenen, die unabhängig von einem Diabetes mellitus in der Schwangerschaft bestand $(24,37,60,85)$. Laut einer aktuellen Metaanalyse hatten adipöse Schwangere ein 2,4-fach erhöhtes Risiko, ein LGA-Kind zu gebären, im Vergleich zu Frauen mit Normalgewicht (85). Sebire et al. fanden die Rate der LGA-Neugeborenen unter übergewichtigen und adipösen Müttern mit 13,4\% bzw. 17,5\% signifikant erhöht gegenüber normalgewichtigen Frauen mit 9,0\% (37). Diese Ergebnisse sind in etwa mit den hier vorliegenden Prozentangaben vergleichbar. Auch in der HAPO-Studie ( $\mathrm{N}=23316)$ konnte gezeigt werden, dass mit steigendem BMI der Frau, unabhängig vom Blutglukosewert, das Risiko für ein als LGA eingestuftes Kind signifikant zunimmt. Zudem trat die 
neonatale Hyperinsulinämie, definiert als C-Peptid-Wert oberhalb der 90. Perzentile im Nabelschnurblut, mit steigendem mütterlichen BMI, unabhängig von einer diabetischen Stoffwechsellage der Schwangeren, signifikant häufiger auf (60). Diese Beobachtungen deuten darauf hin, dass es neben einem erhöhten mütterlichen Blutglukosewert (siehe 1.1.3 Pedersen-Hypothese (49)) andere adipositas-assoziierte Faktoren gibt, die zu einer neonatalen Hyperinsulinämie bzw. zu einem gesteigerten fetalen Wachstum führen (176). Ramsay et al. konnten in ihrer Studie an schwangeren Frauen demonstrieren, dass Adipositas, auch bei ansonsten gesunden Müttern, u. a. mit einer Dyslipidämie einhergeht. Im Vergleich zu normalgewichtigen Schwangeren war die Konzentration der Nüchtern-Triglycerid-Werte bei adipösen Frauen wesentlich erhöht (130). Nach Schäfer-Graf et al. waren erhöhte Konzentrationen der Triglyceride und der freien Fettsäuren im Blut von gut eingestellten, diabetischen Müttern hochsignifikant mit dem Auftreten von LGA-Kindern assoziiert (177).

Die Neugeborenen von übergewichtigen und adipösen Müttern in ihrer Gesamtheit wurden laut den vorliegenden Auswertungen mit jeweils 8,2\% signifikant seltener als SGA eingestuft gegenüber 9,8\% unter den Kindern von Schwangeren mit Normalgewicht. Dabei war die Rate der SGA-Kinder unter Frauen mit Adipositas Grad I mit 7,5\% am niedrigsten, stieg jedoch unter Frauen der Kategorien Adipositas Grad II und III auf 8,8\% bzw. 9,6\% an.

Eine im Vergleich zu normalgewichtigen Frauen signifikant erniedrigte Rate an SGA-Kindern unter übergewichtigen und adipösen Patientinnen bestätigten andere Studien. Dabei wurde, übereinstimmend mit den eigenen Ergebnissen, von der Tendenz eines zunehmenden Anteils von SGA-Kindern mit steigendem BMI in den hohen BMI-Kategorien berichtet $(24,28,37)$. Nach Magann et al. lag die Rate der SGA-Kinder unter normalgewichtigen Patientinnen bei 6,5\%. Im Vergleich dazu wurden die Neugeborenen von übergewichtigen und adipösen Müttern mit 4,2\% bzw. 5,7\% signifikant seltener als SGA eingestuft. Den im Vergleich zu übergewichtigen Frauen höheren Anteil der SGA-Kinder unter fettleibigen Frauen begründeten die Autoren als Folge von adipositas-assoziierten Komorbiditäten wie u. a. der Präeklampsie (24). Sebire et al. gaben SGA-Häufigkeiten von 5,5\% unter normalgewichtigen, 4,6\% unter übergewichtigen und 4,8\% unter adipösen Schwangeren an, wobei Neugeborene mit einem Geburtsgewicht unterhalb der 5. Perzentile als SGA definiert waren (37). Die höchste Rate an SGAKindern war laut Cnattingius et al. sowohl unter Primiparae $(4,5 \%)$ als auch unter Pluriparae $(2,7 \%)$ mit einem BMI $<19 \mathrm{~kg} / \mathrm{m}^{2} \mathrm{zu}$ beobachten $(28)$. 
Beim Vergleich der kindlichen Apgar-Werte nach einer, fünf und zehn Minuten postnatal wurden in dieser Studie zu allen genannten Zeitpunkten signifikante Unterschiede zwischen den BMI-Gruppen Normalgewicht und Adipositas festgestellt. Die Apgar-Mittelwerte waren bei den Neugeborenen adipöser Frauen mit 8,44, 9,49 bzw. 9,75 nach der ersten, fünften und zehnten Lebensminute signifikant niedriger, verglichen mit denen des Referenzkollektivs mit 8,66, 9,65 bzw. 9,83. Die Kinder fettleibiger Frauen wurden mit 14,1\%, 4,4\% bzw. 1,6\% signifikant häufiger mit einem Apgar-Wert kleiner als acht Punkte nach der ersten, fünften und zehnten Lebensminute bewertet im Vergleich zu den Neugeborenen normalgewichtiger Mütter mit 9,4\%, 2,9\% bzw. 1,0\%. Ein Apgar-Wert kleiner als vier Punkte wurde bei den Kindern adipöser Frauen nach der ersten, fünften und zehnten Lebensminute mit 2,3\%, 0,6\% bzw. 0,5\% signifikant häufiger bestimmt als unter den Neugeborenen der Referenzgruppe mit 1,4\%, 0,3\% bzw. 0,3\%. Diese Ergebnisse sprechen für eine eingeschränkte Vitalität der Neugeborenen adipöser Frauen, verglichen mit denen normalgewichtiger Frauen in den ersten zehn Lebensminuten.

Auch in anderen Studien wurden signifikant niedrigere Apgar-Werte in den ersten zehn Lebensminuten unter den Kindern adipöser Frauen im Vergleich zu denen normalgewichtiger Mütter beschrieben (29, 34, 37, 64, 86, 87). Laut den Ergebnissen von Raja et al. (N=27668) hatten die Kinder von übergewichtigen ( $\mathrm{aOR}=1,2$ bzw. 1,4), adipösen (BMI 30,0-39,9 kg/m²) $\left(\mathrm{aOR}=1,5\right.$ bzw. 1,6) und stark adipösen Frauen (BMI $\left.\geq 40,0 \mathrm{~kg} / \mathrm{m}^{2}\right)(\mathrm{aOR}=1,7 \mathrm{bzw} .1,5)$ ein signifikant erhöhtes Risiko für Apgar-Werte kleiner als acht Punkte nach der ersten und fünften Lebensminute im Vergleich zu den Kindern von Frauen mit Normalgewicht (29). Ein systematischer Review von Zhu et al. analysierte die Ergebnisse von elf Kohortenstudien im Zeitraum von 2008 bis 2015. Dabei konnte ein signifikant erhöhtes Risiko für einen kindlichen ApgarWert unterhalb von sieben Punkten nach der ersten $(\mathrm{aOR}=1,3)$ und fünften Lebensminute $(\mathrm{aOR}=1,4)$ sowie unterhalb von drei Punkten nach der fünften Lebensminute $(\mathrm{aOR}=1,4)$ für Kinder adipöser Mütter im Vergleich zu denen normalgewichtiger Frauen festgestellt werden (86). In der Studie von Persson et al. wurden knapp 1,8 Millionen Geburten mit einem Schwangerschaftsalter $\geq 37$ SSW im Zeitraum von 1992 bis 2010 basierend auf den Daten des sog. „Medical Birth Registers“ von Schweden untersucht. Dabei nahm das Odds Ratio für kindliche Apgar-Werte unterhalb von vier Punkten im Vergleich zur Referenzgruppe Normalgewicht mit steigender BMI-Kategorie der Mutter von Übergewicht bis Adipositas Grad III sowohl für die Werte nach der fünften (OR 1,5-3,8) als auch nach der zehnten Lebensminute (OR 1,3-2,7) signifikant zu. Auch nach Adjustierung für Confounder wie mütterliches Alter, Parität, Rauchverhalten und Geburtsmodus sowie in der Analyse nach Ausschluss der Frauen mit diabetischer 
Stoffwechsellage, hypertensiven Erkrankungen in der Schwangerschaft und fehlgebildeten Kindern war statistische Signifikanz zu beobachten (87). Als mögliche Ursachen nannten die Autoren das gehäufte Auftreten einer Schulterdystokie unter der Geburt adipöser Frauen sowie die neonatale Hyperinsulinämie, die laut den Ergebnissen der HAPO-Studie auch bei nichtdiabetischen Frauen stark mit maternaler Adipositas assoziiert war (60). Zudem könnte es laut Persson et al. durch adipositas-bedingte Veränderungen im mütterlichen Organismus (u. a. chronische Entzündung, endotheliale Dysfunktion) zu einer verminderten Durchblutung der Plazenta adipöser Frauen kommen, was das Risiko für eine fetale Asphyxie unter der Geburt erhöht (87).

Im Unterschied zu den in dieser Studie beschriebenen Ergebnissen konnte Sheiner et al. und Kiran et al. zufolge kein statistisch signifikanter Unterschied bzgl. eines Apgar-Werts unterhalb von sieben Punkten nach der ersten und fünften Lebensminute zwischen den Neugeborenen adipöser (BMI $\left.\geq 30 \mathrm{~kg} / \mathrm{m}^{2}\right)$ und nicht-adipöser Frauen $\left(\mathrm{BMI}<30 \mathrm{~kg} / \mathrm{m}^{2}\right)$ gefunden werden, wobei Frauen mit einer hypertensiven Erkrankungen oder diabetischen Stoffwechsellage in der Schwangerschaft in den jeweiligen Studienkollektiven nicht eingeschlossen waren $(25,42)$.

In der vorliegenden Untersuchung war weiterhin ein signifikanter Zusammenhang zwischen mütterlicher Adipositas und der Häufigkeit einer kindlichen Azidose zu beobachten. Neugeborene fettleibiger Frauen unterschieden sich mit einem mittleren $\mathrm{pH}-$ Wert im Nabelarterienblut von 7,267 signifikant von denen normalgewichtiger Frauen mit 7,272. Im Analysezeitraum waren insgesamt 653 Fälle $(2,4 \%)$ eines kindlichen $\mathrm{pH}$-Werts unterhalb von 7,1 und darunter 75 Fälle $(0,3 \%)$ eines kindlichen $\mathrm{pH}-$ Werts unterhalb von 7,0 zu verzeichnen. Die Neugeborenen adipöser Frauen wiesen mit 3,1\% (97 Fälle) bzw. 0,5\% (16 Fälle) signifikant häufiger einen pH-Wert kleiner als 7,1 bzw. 7,0 auf, als die Kinder der Frauen des Referenzkollektivs mit 2,1\% (401 Fälle) bzw. 0,2\% (43 Fälle).

Stepan et al. analysierten die Daten von 5067 Frauen, die im Zeitraum von 2001 bis 2004 in der Universitätsklinik Leipzig einen Einling entbanden. In Übereinstimmung mit den Ergebnissen der hier vorliegenden Studie war ein $\mathrm{pH}$-Wert unterhalb von 7,1 bei den Kindern adipöser Frauen gegenüber der normalgewichtigen Vergleichsgruppe signifikant öfter zu beobachten. Die Häufigkeit stieg ausgehend von der BMI-Kategorie Normalgewicht mit 1,9\% unter den Kindern von Frauen mit Adipositas Grad I bis III auf 3,7\%, 3,5\% bzw. 7,6\% an (64). In der Studie von Magann et al. (N=4490) wiesen die Kinder adipöser Frauen mit 3,9\% (46 Fälle) in der univariaten Analyse signifikant häufiger einen pH-Wert unterhalb von 7,1 auf als die Kinder 
des normalgewichtigen Referenzkollektivs mit 2,8\% (55 Fälle). Nach Adjustierung für Confounder wie u. a. Gestationsalter, metabolische und hypertensive Erkrankungen in der Schwangerschaft, Parität und Geburtsmodus war der Unterschied zwischen den BMI-Gruppen nicht mehr statistisch signifikant (71). Auch andere Autoren konnten die maternale Adipositas nicht als unabhängigen Risikofaktor für eine Azidose des Neugeborenen identifizieren (29, 42, 67 , 86).

Bei den Kindern adipöser Frauen wurde in dieser Studie mit einem Mittelwert von -3,99 mmol/1 ein signifikant niedrigeres Basendefizit im Nabelarterienblut gemessen als bei Neugeborenen normalgewichtiger Mütter mit $-4,15 \mathrm{mmol} / \mathrm{l}$. In der Untersuchung von Raja et al. wurden die Daten von 27668 Frauen analysiert, die im Zeitraum von 2002 bis 2007 am Northwick Park Hospital in London entbanden. Dabei war das Risiko für eine kindliche metabolische Azidose mit einem BE-Wert kleiner als $-12 \mathrm{mmol} / \mathrm{l}$ für Frauen mit einem BMI $\geq 30 \mathrm{~kg} / \mathrm{m}^{2}$ signifikant erhöht gegenüber Müttern mit einem BMI $<30 \mathrm{~kg} / \mathrm{m}^{2}(\mathrm{aOR}=2,5)$. Das Odds Ratio wurde adjustiert für mütterliches Alter, Ethnizität, Parität und Rauchverhalten. Beim Vergleich der BMIGruppen, eingeteilt in Übergewicht (BMI 25,0-29,9 kg/m²), Adipositas (BMI 30,0-39,9 kg/m²), starke Adipositas $\left(B M I \geq 40 \mathrm{~kg} / \mathrm{m}^{2}\right.$ ) und Normalgewicht als Referenzgruppe (BMI 18,5-24,9 $\mathrm{kg} / \mathrm{m}^{2}$ ), waren keine statistisch signifikanten Unterschiede zwischen den Gruppen zu beobachten (29).

In der Klinik St. Hedwig wiesen im Untersuchungszeitraum 1,9\% der Kinder (537 Fälle) eine Fehlbildung auf, wobei kein signifikanter Zusammenhang mit dem BMI der Mutter hergestellt werden konnte. Unter den Kindern normalgewichtiger Frauen waren 1,9\% von einer kongenitalen Anomalie betroffen, unter übergewichtigen Frauen war der Anteil vergleichsweise mit 2,2\% etwas höher und unter adipösen Frauen mit 1,8\% etwas niedriger.

Auch Schummers et al., die Daten von 226958 Schwangerschaften im Zeitraum von 2004 bis 2012 in British Columbia auswerteten, konnten keine signifikante Assoziation zwischen dem maternalen BMI und der Häufigkeit von angeborenen Fehlbildungen beobachten. In den BMIKategorien Normalgewicht, Übergewicht und Adipositas Grad I bis III betrug der Anteil der fehlgebildeten Kinder 4,7\%, 4,5\%, 4,9\%, 5,5\% und 5,1\% (36). Von dem Fehlen eines signifikanten Zusammenhangs zwischen dem BMI der Mutter und einer kongenitalen Anomalie des Kindes berichtete auch eine israelische Studie, der 126080 Geburten im Zeitraum von 1988 bis 2002 zugrunde lagen. Frauen mit hypertensiven Erkrankungen oder Diabetes mellitus waren 
vom untersuchten Studienkollektiv ausgeschlossen. Unter adipösen Frauen (BMI $\geq 30 \mathrm{~kg} / \mathrm{m}^{2}$ ) war in 4,0\% eine Fehlbildung des Kindes zu finden, während der Anteil unter nicht-adipösen Frauen $\left(\mathrm{BMI}<30 \mathrm{~kg} / \mathrm{m}^{2}\right)$ bei 3,9\% lag (25). Eine US-amerikanische Kohortenstudie von Feldmann et al. ( $\mathrm{N}=72915)$ konnte keine statistisch signifikante Beziehung zwischen maternaler Adipositas und der Fehlbildungsrate bei den Nachkommen in Bezug auf Neuralrohrdefekte aufzeigen (178).

Im Gegensatz dazu identifizierten zahlreiche Studien die Adipositas der Mutter als bedeutenden Risikofaktor für kongenitale Anomalien (32, 88-96). In der prospektiven Fall-Kontroll-Studie von Queißer-Luft et al. an 20248 Neugeborenen des Mainzer Geburtenregisters zeigte sich ein 1,3-fach erhöhtes Fehlbildungsrisiko für die Kinder adipöser Frauen im Vergleich zu einem normalgewichtigen Referenzkollektiv (88). In Übereinstimmung mit den Befunden verschiedener Autoren (89-93) erwies sich das Risiko für Neuralrohrdefekte $(\mathrm{OR}=1,9)$ und speziell die Spina bifida $(\mathrm{OR}=2,2)$ in einer groß angelegten Metaanalyse von Stothard et al. für adipöse Frauen signifikant erhöht gegenüber Frauen mit Normalgewicht (94). Analog zu den Beobachtungen anderer Studien $(91,92,96)$ hatten die Kinder von adipösen Frauen ein erhöhtes Risiko für kardiovaskuläre Fehlbildungen insgesamt $(\mathrm{OR}=1,3)$ sowie speziell für septale Anomalien $(\mathrm{OR}=1,2)$ (94). Eine Zunahme des Risikos für eine Lippen-Kiefer-Gaumenspalte $(\mathrm{OR}=1,2)$ bei den Kindern adipöser Frauen, verglichen mit denen normalgewichtiger Patientinnen, entsprach dem Befund einer großen (N=988171) prospektiven Studie aus Schweden (95). Zudem waren die Fehlbildungen anorektale Atresie $(\mathrm{OR}=1,5)$, Hydrozephalus $(\mathrm{OR}=1,7)$ und Reduktionsdefekte der Extremitäten $(\mathrm{OR}=1,3)$ bei den Kindern adipöser Frauen signifikant häufiger zu beobachten als bei den Neugeborenen normalgewichtiger Mütter (92, 94). Das Risiko für eine Gastroschisis $(\mathrm{OR}=0,2)$ fanden Stothard et al., in Übereinstimmung mit den Befunden anderer Autoren (92, 179), für die Kinder adipöser gegenüber normalgewichtiger Frauen signifikant reduziert (94). In einer großen Fall-Kontroll-Studie von Waller et al. $(\mathrm{N}=14314)$ zeigte sich zudem für die Neugeborenen adipöser Mütter ein 1,3-fach, 1,4-fach bzw. 1,6-fach erhöhtes Risiko für Hypospadien zweiten oder dritten Grades, Zwerchfellhernien und Omphalozelen, verglichen mit normalgewichtigen Frauen. Schwangere mit diabetischer Stoffwechsellage waren von dem untersuchten Studienkollektiv ausgeschlossen, da präkonzeptioneller Diabetes mellitus $(144,145)$ bzw. Gestationsdiabetes mellitus $(31,93)$ per se Risikofaktoren für eine kindliche Fehlbildung darstellen (92). 
Auf welche Weise der mütterliche BMI Einfluss auf die Fehlbildungsrate bei den Nachkommen nimmt, ist unklar. Obwohl maternale Adipositas gehäuft mit einem Diabetes mellitus einhergeht, sind beide unabhängige Risikofaktoren für kindliche kongenitale Anomalien $(88,92,93)$. Undiagnostizierte Fälle eines Diabetes mellitus in der Frühschwangerschaft adipöser Frauen werden als ursächlich für die erhöhte Fehlbildungsrate der Kinder adipöser Frauen diskutiert (92, 94, 96). Ein mütterlicher Folsäuremangel stellt einen Risikofaktor für Neuralrohrdefekte der Nachkommen dar. Den Ergebnissen verschiedener Studien zufolge nahm jedoch das Risiko für kindliche Neuralrohrdefekte mit zunehmendem maternalen BMI, unabhängig vom präkonzeptionellen Angebot an Folat in der Nahrung, zu (89, 92). Mithilfe des sog. „Nicht-invasiven pränatalen Testings (NIPT)“ im ersten Trimenon ist eine Beurteilung des Risikos für Chromosomenstörungen anhand der im maternalen Blut zirkulierenden, zellfreien fetalen DNA (cffDNA) möglich. Ein Anteil der cffDNA an der gesamten, im maternalen Blut zirkulierenden, freien DNA über 4\% gilt als Voraussetzung für ein verlässliches Ergebnis. Die diagnostische Sicherheit des NIPT ist bei adipösen Schwangeren vermindert, da die fetale DNA-Fraktion im maternalen Blut mit zunehmendem Gewicht der Schwangeren abnimmt. Einen cffDNA-Anteil kleiner 4\% wiesen laut Ashoor et al. in der 12. SSW bei einem Gewicht von $60 \mathrm{~kg} \mathrm{0,7 \%} \mathrm{der}$ Frauen auf, während der Anteil bei $160 \mathrm{~kg}$ auf 51,1\% stieg (180). Das vermehrte Fettgewebe in der Bauchdecke adipöser Schwangerer absorbiert und streut den Ultraschallstrahl, was in einer Verminderung der Bildqualität resultiert (173). Die pränatale Detektion einer kindlichen Fehlbildung per Ultraschall nahm laut der Untersuchung von Dashe et al. mit zunehmendem BMI der Schwangeren ab (181). Eine durch mangelnde pränatale Diagnose verminderte Rate an Schwangerschaftsabbrüchen aufgrund von fetalen Anomalien könnte die erhöhte Prävalenz einer Fehlbildung bei den Neugeborenen adipöser Frauen erklären (94). Der Anteil der pränatal diagnostizierten fetalen Anomalien im Standard-Ultraschall betrug laut Dashe et al. im zweiten Trimenon 66\%, 49\%, 48\%, 42\% bzw. 25\% unter normalgewichtigen, übergewichtigen und adipösen Schwangeren der Schweregrade I bis III. Auch mithilfe eines zielgerichteten Ultraschalls war eine Abnahme des Anteils der pränatal festgestellten Fehlbildungen mit steigendem mütterlichen BMI zu beobachten. Die Prozentwerte lagen bei 97\%, 91\%, 75\%, 88\% bzw. 75\% für normalgewichtige, übergewichtige und fettleibige Mütter der Schweregrade I bis III (181).

In der Klinik St. Hedwig war der Anteil der Fehlbildungen, die sonografisch festgestellt werden konnten, unter übergewichtigen Frauen mit 68,5\% am höchsten, gefolgt von normalgewichtigen Schwangeren mit 63,5\% und schließlich Patientinnen mit einem BMI $\geq 30 \mathrm{~kg} / \mathrm{m}^{2}$, unter denen die Rate der Anomalien mit pränataler Diagnose bei 53,6\% lag. 
In der vorliegenden Studie nahm die Rate der kindlichen Verlegungen auf eine neonatologische Station mit steigendem BMI der Mutter zu. Der Anteil der Frauen, deren Neugeborene zur intensiven Betreuung verlegt werden mussten, war in der BMI-Kategorie Übergewicht und Adipositas mit 17,5\% bzw. 21,1\% signifikant höher als in der Referenzgruppe mit 14,8\%.

Die Ergebnisse zahlreicher Autoren bestätigten eine positive Assoziation zwischen dem maternalen BMI und der Notwendigkeit einer kindlichen Verlegung $(29,32,36,37,42,70)$. Sebire et al. gaben ein 1,2-fach bzw. 1,3-fach erhöhtes Risiko für eine Verlegung auf die neonatologische Station für die Neugeborenen übergewichtiger und adipöser Frauen im Vergleich zu denen normalgewichtiger Mütter an (37). Laut einer kanadischen Studie, der die Daten von über 226000 Geburten im Zeitraum von 2004 bis 2012 zugrunde lagen, bedurften die Kinder von übergewichtigen (4,2\%) und adipösen Frauen der Schweregrade I bis III (4,7\%, 4,9\%, 6,0\%) signifikant häufiger der Betreuung auf einer Intensivstation für mindestens 48 Stunden als die Neugeborenen normalgewichtiger Frauen (3,9\%) (36). In Übereinstimmung mit den Befunden von Callaway et al. (32) wurde der Verlegungsgrund Hypoglykämie bei Diabetes mellitus der Mutter in der vorliegenden Studie bei den Kindern von adipösen Frauen mit 15,5\% signifikant häufiger genannt als bei denen des Referenzkollektivs mit 7,4\%.

Die perinatale Mortalität lag im Untersuchungszeitraum mit insgesamt 180 Fällen bei $0,6 \%$. In Bezug auf den maternalen BMI war der Anteil unter den Kindern adipöser Frauen mit 1,0\% signifikant höher als im Referenzkollektiv mit 0,6\%. Dabei war eine signifikante Häufung der Fälle eines IUFT $(n=79)$ bei adipösen $(0,5 \%)$ gegenüber normalgewichtigen Schwangeren $(0,3 \%)$ zu beobachten. Hinsichtlich des Versterbens des Fetus sub partu $(n=42)$ waren keine signifikanten Unterschiede zwischen den BMI-Kategorien Normalgewicht $(0,2 \%)$, Übergewicht $(0,1 \%)$ und Adipositas $(0,2 \%)$ festzustellen. Das Gestationsalter der subpartual verstorbenen Feten betrug in $80,9 \%$ der Fälle $<37+0$ SSW und davon in 59,5\% der Fälle $<27+0$ SSW. Ein neonataler Tod innerhalb der ersten sieben Lebenstage $(n=59)$ war bei den Kindern adipöser Frauen $(0,4 \%)$, im Vergleich zu denen normalgewichtiger Frauen $(0,2 \%)$, signifikant häufiger zu verzeichnen. Dabei fielen $88,1 \%$ der neonatalen Todesfälle auf eine Tragzeit $<37+0$ SSW.

Auch den Befunden anderer Autoren zufolge war die maternale Adipositas mit einer Erhöhung der perinatalen Mortalität assoziiert (24, 26, 63, 97). Nach Angaben einer 38 Studien umfassenden Metaanalyse aus dem Jahr 2014 stieg das relative Risiko der perinatalen Mortalität ausgehend von normalgewichtigen Frauen pro Anstieg um fünf BMI-Einheiten jeweils um das 1,3fache an (26). 
Die Wahrscheinlichkeit einer Totgeburt war laut den Ergebnissen mehrerer Studien bei adipösen Schwangeren um den Faktor 1,4 bis 2,6 erhöht gegenüber Frauen mit Normalgewicht (26, 28, 34, 36, 37, 74, 182-184). In Übereinstimmung mit den eigenen Arbeitsergebnissen konnte die maternale Adipositas in der aktuellen Literatur als Risikofaktor für einen intrauterinen Fruchttod (26, 62, 64, 97-99), nicht jedoch für den subpartualen Tod des Fetus (26, 97, 98), identifiziert werden. Einer Datenanalyse der Universitätsklinik Leipzig im Zeitraum von 2001 bis 2004 zufolge trat der IUFT unter übergewichtigen (0,6\%) und adipösen Schwangeren der Schweregrade I $(0,5 \%)$, II (1,0\%) und III $(1,3 \%)$ häufiger auf als unter normalgewichtigen Frauen $(0,5 \%)$. Die Unterschiede zwischen den BMI-Gruppen waren nicht statistisch signifikant, was am ehesten auf die geringe Stichprobengröße ( $\mathrm{N}=5067)$ zurückzuführen war (64). Tennant et al. ( $\mathrm{N}=29856)$ beschrieben ein 2,2-fach erhöhtes Risiko für einen IUFT unter adipösen gegenüber normalgewichtigen Schwangeren, wobei die Fälle von kindlichen Fehlbildungen und präexistentem Diabetes mellitus der Mutter vom untersuchten Studienkollektiv ausgeschlossen waren, die per se Risikofaktoren für einen IUFT darstellen (98). Auch nach Adjustierung für mütterliches Alter, Nikotinabusus, Gestationsalter und Präeklampsie blieben die beobachteten Unterschiede zwischen den BMI-Gruppen signifikant bestehen (97). Folglich müsste es, außer dem Einfluss adipositas-assoziierter Komorbiditäten, weitere Mechanismen geben, die dem Zusammenhang zwischen maternaler Adipositas und dem erhöhten Risiko für einen IUFT zugrunde liegen. Adipositas in der Schwangerschaft geht, auch bei ansonsten gesunden Müttern, u. a. mit einer Hypertonie, endothelialer Dysfunktion, Dyslipidämie und chronischer Inflammation einher, was in plazentaren Funktionsstörungen mit der Folge eines IUFT resultieren könnte (130). Pinar et al. fanden in den Plazenten von Totgeburten mit 55,7\% bzw. 30,4\% signifikant häufiger vaskulär-degenerative und entzündliche Veränderungen als in den Plazenten lebendgeborener Kinder mit 0,5\% bzw.12,0\% (185).

Die in dieser Studie beobachtete, signifikante Assoziation zwischen maternaler Adipositas und der erhöhten neonatalen Mortalität wurde von den Befunden anderer Autoren bestätigt (26, 36, 74, 97). In einer kanadischen Studie, die die Daten von 226958 Geburten im Zeitraum von 2004 bis 2012 analysierte, verstarben die Neugeborenen übergewichtiger $(0,5 \%)$ und adipöser Mütter der Schweregrade I (0,6\%), II (0,6\%) und III (0,8\%) signifikant häufiger im Krankenhaus als die Kinder normalgewichtiger Frauen (0,4\%) (36). Tennant et al. fanden das Risiko eines neonatalen Todes in den ersten sieben Lebenstagen für die Nachkommen adipöser Mütter, unabhängig von adipositas-assoziierten Komorbiditäten und kindlichen Fehlbildungen, um den Faktor 2,6 erhöht, verglichen mit den Neugeborenen normalgewichtiger Frauen (97). 


\subsection{Wochenbettverlauf}

In der vorliegenden Studie wurden die Neugeborenen übergewichtiger und adipöser Frauen mit 74,9\% bzw. 67,7\% signifikant seltener voll gestillt als die Kinder normalgewichtiger Wöchnerinnen mit 80,0\%. Mit der maternalen BMI-Kategorie nahm die Rate der voll stillenden Mütter ab und erreichte unter Frauen der BMI-Gruppe Adipositas Grad III ein Minimum von 60,4\%.

Auch die Ergebnisse anderer Autoren zeigten eine Abnahme des Anteils stillender Mütter mit zunehmendem BMI (37, 100-104). Einer US-amerikanischen Studie zufolge war bereits die geplante Dauer des Stillens bei den befragten Schwangeren mit einem BMI $\geq 29 \mathrm{~kg} / \mathrm{m}^{2}$ mit 6,9 Monaten im Mittel signifikant kürzer gegenüber Schwangeren mit einem BMI $<26 \mathrm{~kg} / \mathrm{m}^{2}$ mit 9,3 Monaten (100). Laut einer belgischen Studie stillten 92,0\% der normalgewichtigen Mütter, während der Anteil unter übergewichtigen und adipösen Frauen mit 80,0\% bzw. 68,0\% signifikant geringer war (101). Liu et al. gaben Stillraten von 71,0\%, 66,0\%, 63,8\% bzw. 58,0\% unter normalgewichtigen, übergewichtigen, adipösen (BMI 30,0-34,9 kg/m²) und stark adipösen Frauen $\left(B M I \geq 35 \mathrm{~kg} / \mathrm{m}^{2}\right)$ an (102). Laut der systematischen Übersichtsarbeit von Turcksin et al. war die Wahrscheinlichkeit, primär abzustillen, für adipöse Frauen um den Faktor 1,2 bis 3,7 erhöht gegenüber der normalgewichtigen Vergleichsgruppe. Unter den Frauen, die ihren Kindern die Brust gaben, nahm die mediane Dauer des Stillens mit steigender mütterlicher BMI-Kategorie ab (103).

Nach Guelinckx et al. gaben übergewichtige und adipöse Frauen mit 18,0\% bzw. 24,0\% signifikant häufiger eine zu geringe Milchproduktion als Grund für das vorzeitige Abstillen an im Vergleich zu normalgewichtigen Frauen mit 13,0\% (101). Zudem könnte ein niedriger sozioökonomischer Status dem beschriebenen Zusammenhang zwischen maternaler Adipositas und dem Stillverhalten zugrunde liegen, da er laut verschiedener Studien sowohl mit einer erhöhten Adipositas-Prävalenz $(9,12,38,68,150)$ als auch einer reduzierten Häufigkeit des Stillens (186) assoziiert war. Als weitere Erklärungsansätze für die im Vergleich zu normalgewichtigen Müttern geringere Stillquote unter adipösen Müttern gelten die oftmals mit Adipositas einhergehenden großen Mammae mit flachen Mamillen, die das Anlegen der Kinder erschweren, sowie ein durch die Adipositas bedingtes Unbehagen, in der Öffentlichkeit zu stillen (103).

Wie bereits an anderer Stelle dieser Arbeit erwähnt, besteht für die Nachkommen adipöser Mütter ein lebenslang erhöhtes Risiko für Adipositas und deren Folgeerkrankungen (siehe 1.1.4). Da das Stillen als optimale Ernährung für Neugeborene, neben anderen positiven Effekten für 
Mutter und Kind, das Adipositas-Risiko der Nachkommen vermindert (siehe 1.1.3), sollten insbesondere adipöse Frauen über die Vorteile der Muttermilch informiert und das Stillen des Säuglings angeraten werden. Ein intensive Stillvorbereitung und -begleitung von adipösen Frauen kann helfen, Stillschwierigkeiten und einem vorzeitigen Abstillen vorzubeugen.

Eine revisionsbedürftige Wundheilungsstörung bzw. speziell die Wundinfektion nach Sectio caesarea trat in dieser Studie bei Wöchnerinnen mit einem BMI $>40 \mathrm{~kg} / \mathrm{m}^{2}$ mit $0,7 \%$ bzw. 1,2\% signifikant häufiger auf als unter normalgewichtigen Frauen mit 0,1\% bzw. 0,2\%. Beim Vergleich der Patientinnen mit einem BMI von $25,0 \mathrm{~kg} / \mathrm{m}^{2}$ bis $29,9 \mathrm{~kg} / \mathrm{m}^{2} \mathrm{bzw}$. $\geq 30 \mathrm{~kg} / \mathrm{m}^{2}$ mit der Referenzgruppe waren keine statistisch signifikanten Zusammenhänge bzgl. der genannten Ereignisse zu beobachten. Auch andere Autoren fanden eine positive Assoziation zwischen einem hohen maternalen BMI und der Häufigkeit einer Wundinfektion in Wochenbett $(37,70,71,81$, 105, 106). Den Ergebnissen einer Studie zufolge, die das Outcome von 287213 Schwangerschaften in London im Zeitraum von 1989 bis 1997 untersuchte, trat die Wundinfektion sowohl unter übergewichtigen als auch unter adipösen Wöchnerinnen mit 0,6\% bzw. 1,3\% signifikant häufiger auf als bei normalgewichtigen Frauen mit 0,4\% (37). Myles et al. identifizierten die maternale Adipositas als unabhängigen Risikofaktor für eine Wundinfektion und eine Endometritis im Wochenbett nach der Entbindung per Sectio caesarea. Dieser Zusammenhang war sowohl in der Gruppe der Wöchnerinnen nach nicht-elektiver Sectio caesarea als auch in der Gruppe der Frauen nach elektiver Sectio caesarea mit prophylaktischer Gabe von Antibiotika zu beobachten (105).

Adipöse Wöchnerinnen entwickelten in dem dieser Studie zugrundeliegenden Kollektiv mit 1,2\% signifikant häufiger postpartales Fieber als normalgewichtigen Frauen, unter denen der Anteil bei 0,5\% lag. Auch Sebire et al. berichteten von einer signifikanten Häufung der Fälle von Fieber im Wochenbett bei übergewichtigen (1,3\%) und adipösen Frauen (1,5\%) im Vergleich zur Referenzgruppe (1,0\%) (37). In einer israelischen Studie wurden 126080 Geburten analysiert, wobei Schwangere mit hypertensiven Erkrankungen oder Diabetes mellitus von der Analyse ausgeschlossen waren. Dabei trat postpartales Fieber unter den Patientinnen mit einem $\mathrm{BMI} \geq 30 \mathrm{~kg} / \mathrm{m}^{2}$ mit $0,9 \%$ häufiger auf, als unter den Wöchnerinnen mit einem BMI $<30 \mathrm{~kg} / \mathrm{m}^{2}$ mit $0,6 \%$. Der Unterschied zwischen den Gruppen war mit $\mathrm{p}=0,06$ nicht statistisch signifikant (25). 
In einer groß angelegten Studie, in der das Outcome von 287213 Schwangerschaften in London analysiert wurde, lag bei übergewichtigen und adipösen Frauen mit 9,2\% bzw. 7,7\% signifikant seltener eine Anämie im Wochenbett vor als bei normalgewichtigen Frauen mit 10,3\% (37). Konje et al. fanden prozentual eine niedrigere Rate an postpartalen Anämien unter adipösen Frauen mit 8,1\% im Vergleich zu normalgewichtigen Frauen mit 12,2\% (106). Auch in der hier vorliegenden Studie wurde im Vergleich zur Referenzgruppe mit 18,6\% unter adipösen Wöchnerinnen mit 17,6\% prozentual seltener eine Anämie festgestellt, wobei der Unterschied nicht auf statistischer Signifikanz beruhte. Hinsichtlich der Häufigkeit einer bereits antepartal bestehenden Anämie berichteten Garbaciak et al. von einer signifikant niedrigeren Rate unter adipösen und stark adipösen Frauen mit 3,2\% bzw. 2,4\% im Vergleich zu normalgewichtigen Schwangeren mit 4,3\% (63). 


\subsection{Prävention durch gesunde Ernährung und Sport in der Schwangerschaft}

Das maternale und neonatale Outcome kann durch eine ausgewogene Ernährung und regelmäBige Bewegung in der Schwangerschaft zum Teil verbessert werden (187-189), wovon insbesondere Frauen mit präkonzeptionellem Übergewicht bzw. Adipositas profitieren könnten.

Frauen, die sich während der Schwangerschaft an eine Diät hielten, konnten laut den Ergebnissen einer 44 randomisiert-kontrollierte Studien umfassenden Metaanalyse aus dem Jahr 2012 die Gewichtszunahme in der Schwangerschaft im Mittel um 3,84 kg reduzieren. Das relative Risiko für die Komplikationen Gestationshypertonie ( $R R=0,30)$, Schulterdystokie $(R R=0,38)$, GDM (RR=0,39), Präeklampsie ( $R R=0,67)$ und Frühgeburt $(R R=0,68)$ war in der Gruppe der Schwangeren mit Einhaltung einer ausgewogenen Ernährung signifikant reduziert. Ein Effekt der Diät auf die Häufigkeit von SGA-Neugeborenen war nicht zu verzeichnen (187).

Regelmäßige sportliche Betätigung in der Schwangerschaft konnte laut der Untersuchung von Thangaratinam et al. die Gewichtszunahme der Frauen im Mittel um 0,72 kg reduzieren. Zudem war eine signifikante Verringerung des kindlichen Geburtsgewichts um $60 \mathrm{~g}$ im Mittel sowie ein Trend in Richtung niedrigerer LGA-Raten bei regelmäßiger körperlicher Bewegung der Schwangeren zu beobachten (187). Eine neun Studien einschließende Metaanalyse aus dem Jahr 2016 untersuchte den Effekt eines 30 bis 60-minütigen aeroben Ausdauertrainings, drei bis sieben Mal pro Woche, während der Schwangerschaft unter übergewichtigen und adipösen Frauen $\left(B M I \geq 25 \mathrm{~kg} / \mathrm{m}^{2}\right.$ ). Dabei konnte das relative Risiko für die Komplikationen GDM $(\mathrm{RR}=0,61)$ und Frühgeburt $(\mathrm{RR}=0,62)$ in der Interventionsgruppe signifikant reduziert werden (188).

In der sog. FeLIPO-Studie (,Feasibility of a lifestyle intervention programme to prevent obesity in the offspring") wurde der Effekt einer Lebensstilberatung in der Schwangerschaft auf die Gewichtszunahme der Frauen und die Gesundheit von Mutter und Kind in einem cluster-randomisierten Design in acht Frauenarztpraxen im Raum München untersucht (N=250). Die Intervention bestand aus zwei Beratungsgesprächen von speziell geschulten Praxismitarbeitern und Hebammen zu den Themen gesunde Ernährung, Sport und Gewichtsmonitoring während der Schwangerschaft. In der Interventionsgruppe war der Anteil der Schwangeren, die die empfohlene Gewichtszunahme des IOM überschritten, mit 38,2\% signifikant niedriger als in der Kontrollgruppe mit 59,5\%. In Übereinstimmung mit den Befunden von Thangaratinam et al. (187) war die Reduktion der Gewichtszunahme mehr dem Effekt einer geringeren Energiezufuhr als dem einer Steigerung der körperlichen Aktivität zuzuschreiben (189). 
Auf der Basis der Pilotstudie FeLIPO wurde von 2013 bis 2015 das sog. Projekt „Gesund leben in der Schwangerschaft (GeliS)“ in über 70 Frauenarztpraxen in Bayern durchgeführt (N=2286). Prof. Dr. med. Hans Hauner von der Technischen Universität München leitete die Studie in Zusammenarbeit mit dem Kompetenzzentrum für Ernährung (KErn). In der Interventionsgruppe wurde den Patientinnen im Rahmen der Routinevorsorge vier Sitzungen einer gesundheitsförderlichen Lebensstilberatung angeboten, wobei drei davon während der Schwangerschaft und eine nach der Geburt des Kindes stattfanden (190). Die Auswertungen der Studie zeigten, dass die Beratungsgespräche wider Erwarten keinen Effekt auf die Gewichtszunahme in der Schwangerschaft hatten. Trotz der Teilnahme am Programm überschritten $45,1 \%$ der Frauen die vom IOM empfohlene Gewichtszunahme in der Schwangerschaft gegenüber 45,7\% in der Kontrollgruppe. Auch die Häufigkeit eines GDM konnte durch die Beratungsgespräche nicht signifikant verringert werden $(10,8 \%$ in der Interventions- gegenüber $11,1 \%$ in der Kontrollgruppe). Allerdings führten die Gesprächstermine zu dem beabsichtigten gesünderen Lebensstil der Frauen. Ein positiver Effekt war auch hinsichtlich des Geburtsgewichts der Kinder von Frauen mit einer Lebensstilberatung in der Schwangerschaft zu verzeichnen, es war mit 3313 g im Mittel niedriger als in der Kontrollgruppe mit 3363 g. Außerdem war die Rate der Geburtseinleitungen in der Interventionsgruppe gegenüber dem Vergleichskollektiv reduziert $(17,3 \%$ gegenüber 23,5\%) (191, 192). Auch die Ergebnisse des Ein-Jahres-Follow-Ups $(\mathrm{N}=1998)$ untermauerten den Nutzen der in die Routineversorgung eingebetteten Gesprächstermine. Frauen, die der Interventionsgruppe angehörten, hatten ein Jahr nach der Geburt im Mittel mehr Gewicht abgenommen als die Kontrollgruppe (14,3 kg gegenüber 13,4 kg). Zudem wirkte sich die mütterliche Lebensstilberatung positiv auf das Stillverhalten aus. Der Anteil der Frauen, die ihre Kinder voll stillten war in der Interventionsgruppe mit 87,4\% höher als in der Kontrollgruppe mit 84,4\% (193).

Auch mit Hilfe von kostenlos erhältlichen Apps soll Schwangeren und jungen Familien wissenschaftlich geprüfte, aktuelle und kompakte Information zu einer gesunden Lebensweise vermittelt werden. Im Auftrag des Bundesministeriums für Ernährung und Landwirtschaft hat das KErn in Zusammenarbeit mit dem „Netzwerk Gesund ins Leben“ und der „Stiftung Kindergesundheit“ die App-Trilogie „Schwanger \& Essen“, „Baby \& Essen“ und „Kind \& Essen“ entwickelt. Die Apps liefern wichtige Informationen zu ausgewogener Ernährung und körperlicher Aktivität in der Schwangerschaft, im Säuglingsalter und der frühen Kindheit (194). 


\section{ZUSAMMENFASSUNG}

In dieser retrospektiven Studie zum Thema Adipositas in der Schwangerschaft wurden Frauen der BMI-Kategorien Normalgewicht (Referenzgruppe), Übergewicht und Adipositas - gemessen am BMI bei Erstuntersuchung - hinsichtlich Schwangerschafts-, Geburts- und Wochenbettverlauf sowie kindlichem Outcome verglichen. Als Datengrundlage dienten 27754 Einlingsgeburten in der Klinik St. Hedwig in Regensburg im Zeitraum von 2000 bis 2015.

Die Prävalenz von Übergewicht und Adipositas in der Schwangerschaft nahm im Untersuchungszeitraum zu und erreichte jeweils ein Maximum im Jahr 2015.

Übergewichtige und adipöse Schwangere waren gegenüber der Referenzgruppe signifikant häufiger von präexistentem- und Gestationsdiabetes mellitus, chronischer- und Gestationshypertonie, Präeklampsie und Aborten betroffen. Der Anteil der Frauen mit exzessiver Gewichtszunahme und Nikotinabusus in der Schwangerschaft nahm in den hohen BMI-Kategorien zu. Die Häufigkeit von Geburtseinleitungen sowie primären und sekundären Kaiserschnitten war unabhängig von der Parität und adipositas-assoziierten Komorbiditäten der Schwangeren positiv mit dem BMI assoziiert. Die Rate der Komplikationen Schulterdystokie, grünes Fruchtwasser und Geburtsstillstand in der EP war unter adipösen Frauen signifikant erhöht. Maternale Fettleibigkeit wurde sowohl mit der Frühgeburtlichkeit als auch der zeitlichen Übertragung der Schwangerschaft positiv in Verbindung gebracht. Die Neugeborenen der Frauen hoher BMIKategorien waren im Mittel signifikant schwerer, wurden unabhängig von einer diabetischen Stoffwechsellage der Mutter häufiger als LGA und seltener als SGA eingestuft. Die Rate der mittel- und schwergradigen Depressionen in den ersten zehn Lebensminuten, der fortgeschrittenen und schweren Azidosen, der Verlegungen auf die neonatologische Station und der perinatalen Mortalität war unter den Kindern adipöser Patientinnen signifikant gesteigert. Hinsichtlich des Auftretens kongenitaler Anomalien bestand kein signifikanter Zusammenhang mit dem maternalen BMI. Die Rate voll stillender Mütter sank mit steigender BMI-Kategorie. Adipöse Wöchnerinnen waren signifikant häufiger von postpartalem Fieber und bei einem BMI über 40 $\mathrm{kg} / \mathrm{m}^{2}$ von einer revisionsbedürftigen Wundheilungsstörung betroffen als Frauen mit Normalgewicht.

Für die Nachkommen adipöser Mütter besteht durch intrauterine Prägung ein lebenslang erhöhtes Adipositas-Risiko. Um negative Folgen von Adipositas in der Schwangerschaft zu vermeiden und den generationenübergreifenden Teufelskreis der Adipositas zu durchbrechen, sollte adipösen Frauen dringend angeraten werden, ihr Gewicht präkonzeptionell zu reduzieren. 


\section{LITERATURVERZEICHNIS}

1. Benecke A, Vogel H, editors. Übergewicht und Adipositas. Geänd. Aufl. Berlin: Robert Koch-Inst; 2005. (Gesundheitsberichterstattung des Bundes; vol 16).

2. Deutsche Adipositas Gesellschaft (DAG) e.V., Deutsche Diabetes Gesellschaft (DDG), Deutsche Gesellschaft für Ernährung (DGE) e.V., Deutsche Gesellschaft für Ernährungsmedizin (DGEM) e.V. Interdisziplinäre Leitlinie der Qualität S3 zur "Prävention und Therapie der Adipositas": Arbeitsgemeinschaft der Wissenschaftlichen Medizinischen Fachgesellschaften (AWMF); 2014 050/001.

3. Obesity: preventing and managing the global epidemic. Report of a WHO consultation. World Health Organ Tech Rep Ser 2000; 894:i-xii, 1-253.

4. Eckel RH, Grundy SM, Zimmet PZ. The metabolic syndrome. Lancet 2005; 365(9468):1415-28.

5. Grundy SM, Cleeman JI, Daniels SR, Donato KA, Eckel RH, Franklin BA et al. Diagnosis and management of the metabolic syndrome. An American Heart Association/National Heart, Lung, and Blood Institute Scientific Statement. Executive summary. Cardiol Rev 2005; 13(6):322-7.

6. Whitlock G, Lewington S, Sherliker P, Clarke R, Emberson J, Halsey J et al. Body-mass index and cause-specific mortality in 900000 adults: collaborative analyses of 57 prospective studies. Lancet 2009; 373(9669):1083-96.

7. World Health Organization (WHO). Obesity and Overweight: Fact sheet; February 2018 [cited 2019 Aug 7]. Available from: URL: https://www.who.int/news-room/fact-sheets/detail/obesity-and-overweight.

8. Bergmann K.E., Mensink GBM. BGS98: Körpermaße und Übergewicht. Gesundheitswesen 61 (S2) 1999:115-20.

9. Mensink GBM, Schienkiewitz A, Haftenberger M, Lampert T, Ziese T, Scheidt-Nave C. Overweight and obesity in Germany: results of the German Health Interview and Examination Survey for Adults (DEGS1). Bundesgesundheitsblatt Gesundheitsforschung Gesundheitsschutz 2013; 56(5-6):786-94. 
10. Kurth B-M, Schaffrath Rosario A. Die Verbreitung von Ubergewicht und Adipositas bei Kindern und Jugendlichen in Deutschland. Ergebnisse des bundesweiten Kinder- und Jugendgesundheitssurveys (KiGGS). Bundesgesundheitsblatt Gesundheitsforschung Gesundheitsschutz 2007; 50(5-6):736-43.

11. Klein S Dr., Krupka S, Behrendt S, Pulst A, Bleß H-H, editors. Weißbuch Adipositas: Versorgungssituation in Deutschland. Berlin: Medizinisch Wissenschaftliche Verlagsgesellschaft (MWV); 2016.

12. Organisation for Economic Cooperation and Development (OECD). Obesity Update 2017 [cited 2019 Aug 7]. Available from: URL: http://www.oecd.org/health/obesity-update.htm.

13. Flegal KM, Kruszon-Moran D, Carroll MD, Fryar CD, Ogden CL. Trends in Obesity Among Adults in the United States, 2005 to 2014. JAMA 2016; 315(21):2284-91.

14. Flegal KM, Carroll MD, Ogden CL, Johnson CL. Prevalence and trends in obesity among US adults, 1999-2000. JAMA 2002; 288(14):1723-7.

15. Flegal KM, Carroll MD, Ogden CL, Curtin LR. Prevalence and trends in obesity among US adults, 1999-2008. JAMA 2010; 303(3):235-41.

16. Olshansky SJ, Passaro DJ, Hershow RC, Layden J, Carnes BA, Brody J et al. A potential decline in life expectancy in the United States in the 21st century. N Engl J Med 2005; 352(11):1138-45.

17. Institut für Qualität und Patientensicherheit GmbH (BQS). Bundesauswertung Geburtshilfe. Düsseldorf; 2001-2008 [cited 2017 May 24]. Available from: URL: http://www.bqsoutcome.de/2008/ergebnisse/leistungsbereiche/geburtshilfe/index_html.

18. Institut für angewandte Qualitätsförderung und Forschung im Gesundheitswesen GmbH (AQUA). Bundesauswertung Geburtshilfe. Göttingen; 2009-2014 [cited 2019 Aug 7]. Available from: URL: https://sqg.de/front_content.php?idart=119.

19. Institut für Qualitätssicherung und Transparenz im Gesundheitswesen (IQTIG). Bundesauswertung Geburtshilfe. Berlin; 2015 [cited 2019 Aug 7]. Available from: URL: https://iqtig.org/ergebnisse/qs-verfahren/16n1/.

20. Bayerische Arbeitsgemeinschaft für Qualitätssicherung in der stationären Versorgung (BAQ). Jahresauswertung Geburtshilfe: Krankenhaus Barmherzige Brüder Regensburg; 2000-2015. 
21. EURO-PERISTAT Project. European Perinatal Health Report: Health and Care of Pregnant Women and Babies in Europe in 2010; 2010 [cited 2019 Aug 6]. Available from: URL: http://www.europeristat.com/reports.html.

22. GILBERT JAL. The association of maternal obesity, large babies, and diabetes. Br Med J $1949 ; 1(4607): 702-4$.

23. Metwally M, Ong KJ, Ledger WL, Li TC. Does high body mass index increase the risk of miscarriage after spontaneous and assisted conception? A meta-analysis of the evidence. Fertil Steril 2008; 90(3):714-26.

24. Magann EF, Doherty DA, Sandlin AT, Chauhan SP, Morrison JC. The effects of an increasing gradient of maternal obesity on pregnancy outcomes. Aust N Z J Obstet Gynaecol 2013; 53(3):250-7.

25. Sheiner E, Levy A, Menes TS, Silverberg D, Katz M, Mazor M. Maternal obesity as an independent risk factor for caesarean delivery. Paediatr Perinat Epidemiol 2004; 18(3):196-201.

26. Aune D, Saugstad OD, Henriksen T, Tonstad S. Maternal Body Mass Index and the Risk of Fetal Death, Stillbirth, and Infant Death. JAMA 2014; 311(15):1536.

27. Lashen H, Fear K, Sturdee DW. Obesity is associated with increased risk of first trimester and recurrent miscarriage: matched case-control study. Hum Reprod 2004; 19(7):1644-6.

28. Cnattingius S, Bergström R, Lipworth L, Kramer MS. Prepregnancy weight and the risk of adverse pregnancy outcomes. N Engl J Med 1998; 338(3):147-52.

29. Raja UA, Mcaree T, Bassett P, Sharma $S$. The implications of a raised maternal BMI: a DGH experience. J Obstet Gynaecol 2012; 32(3):247-51.

30. Bhat M, K N R, Sarma SP, Menon S, C V S, S GK. Determinants of gestational diabetes mellitus: A case control study in a district tertiary care hospital in south India. Int J Diabetes Dev Ctries 2010; 30(2):91-6.

31. Fadl HE, Ostlund IKM, Magnuson AFK, Hanson USB. Maternal and neonatal outcomes and time trends of gestational diabetes mellitus in Sweden from 1991 to 2003. Diabet Med 2010; 27(4):436-41.

32. Callaway LK, Prins JB, Chang AM, McIntyre HD. The prevalence and impact of overweight and obesity in an Australian obstetric population. Med J Aust 2006; 184(2):56-9. 
33. Solomon CG, Willett WC, Carey VJ, Rich-Edwards J, Hunter DJ, Colditz GA et al. A prospective study of pregravid determinants of gestational diabetes mellitus. JAMA 1997; 278(13):1078-83.

34. Ovesen P, Rasmussen S, Kesmodel U. Effect of prepregnancy maternal overweight and obesity on pregnancy outcome. Obstet Gynecol 2011; 118(2 Pt 1):305-12.

35. Chu SY, Callaghan WM, Kim SY, Schmid CH, Lau J, England LJ et al. Maternal Obesity and Risk of Gestational Diabetes Mellitus. Diabetes Care 2007; 30(8):2070-6.

36. Schummers L, Hutcheon JA, Bodnar LM, Lieberman E, Himes KP. Risk of adverse pregnancy outcomes by prepregnancy body mass index: a population-based study to inform prepregnancy weight loss counseling. Obstet Gynecol 2015; 125(1):133-43.

37. Sebire NJ, Jolly M, Harris JP, Wadsworth J, Joffe M, Beard RW et al. Maternal obesity and pregnancy outcome: a study of 287,213 pregnancies in London. Int J Obes Relat Metab Disord 2001; 25(8):1175-82.

38. Metsala J, Stach-Lempinen B, Gissler M, Eriksson JG, Koivusalo S. Risk of Pregnancy Complications in Relation to Maternal Prepregnancy Body Mass Index: Population-Based Study from Finland 2006-10. Paediatr Perinat Epidemiol 2016; 30(1):28-37.

39. Wang Z, Wang P, Liu H, He X, Zhang J, Yan H et al. Maternal adiposity as an independent risk factor for pre-eclampsia: a meta-analysis of prospective cohort studies. Obes Rev 2013; 14(6):508-21.

40. Salihu HM, La Cruz C de, Rahman S, August EM. Does maternal obesity cause preeclampsia? A systematic review of the evidence. Minerva Ginecol 2012; 64(4):259-80.

41. Molyneaux E, Poston L, Ashurst-Williams S, Howard LM. Obesity and mental disorders during pregnancy and postpartum: a systematic review and meta-analysis. Obstet Gynecol $2014 ; 123(4): 857-67$.

42. Usha Kiran TS, Hemmadi S, Bethel J, Evans J. Outcome of pregnancy in a woman with an increased body mass index. BJOG 2005; 112(6):768-72.

43. Metzger BE, Coustan DR. Summary and recommendations of the Fourth International Workshop-Conference on Gestational Diabetes Mellitus. The Organizing Committee. Diabetes Care 1998; 21 Suppl 2:B161-7. 
44. American Diabetes Association. Gestational diabetes mellitus. Diabetes Care 2004; 27 Suppl 1:90.

45. Metzger BE, Buchanan TA, Coustan DR, Leiva A de, Dunger DB, Hadden DR et al. Summary and recommendations of the Fifth International Workshop-Conference on Gestational Diabetes Mellitus. Diabetes Care 2007; 30 Suppl 2:S251-60.

46. Metzger BE, Lowe LP, Dyer AR, Trimble ER, Chaovarindr U, Coustan et al. Hyperglycemia and adverse pregnancy outcomes. N Engl J Med 2008; 358(19):1991-2002.

47. Kleinwechter H, Bührer C, Hunger-Battefeld W, Kainer F, Kautzky-Willer A, Pawlowski B et al. Diabetes und Schwangerschaft: Evidenzbasierte Leitlinie der Deutschen DiabetesGesellschaft; Klasse S3: Arbeitsgemeinschaft der Wissenschaftlichen Medizinischen Fachgesellschaften (AWMF); 2014 057/023.

48. Cordero L, Landon MB. Infant of the diabetic mother. Clin Perinatol 1993; 20(3):635-48.

49. PEDERSEN J. Diabetes and pregnancy; blood sugar of newborn infants during fasting and glucose administration. Nord Med 1952; 47(30):1049.

50. Report of the National High Blood Pressure Education Program Working Group on High Blood Pressure in Pregnancy. American Journal of Obstetrics and Gynecology 2000; 183(1):S1-S22.

51. Saudan P, Brown MA, Buddle ML, Jones M. Does gestational hypertension become preeclampsia? Br J Obstet Gynaecol 1998; 105(11):1177-84.

52. Broughton PF. Risk factors for preeclampsia. N Engl J Med 2001; 344(12):925-6.

53. Duckitt K, Harrington D. Risk factors for pre-eclampsia at antenatal booking: systematic review of controlled studies. BMJ 2005; 330(7491):565.

54. Backes CH, Markham K, Moorehead P, Cordero L, Nankervis CA, Giannone PJ. Maternal preeclampsia and neonatal outcomes. J Pregnancy 2011; 2011:214365.

55. Khan KS, Wojdyla D, Say L, Gulmezoglu AM, Van Look, Paul F A. WHO analysis of causes of maternal death: a systematic review. Lancet 2006; 367(9516):1066-74.

56. Schausberger CE, Jacobs VR, Bogner G, Wolfrum-Ristau P, Fischer T. Hypertensive Disorders of Pregnancy - A Life-Long Risk?! Geburtshilfe Frauenheilkd 2013; 73(1):47-52. 
57. Ricart W, Lopez J, Mozas J, Pericot A, Sancho MA, Gonzalez N et al. Body mass index has a greater impact on pregnancy outcomes than gestational hyperglycaemia. Diabetologia 2005; 48(9):1736-42.

58. Ehrenberg HM, Durnwald CP, Catalano P, Mercer BM. The influence of obesity and diabetes on the risk of cesarean delivery. American Journal of Obstetrics and Gynecology 2004; 191(3):969-74.

59. Wahabi HA, Fayed AA, Alzeidan RA, Mandil AA. The independent effects of maternal obesity and gestational diabetes on the pregnancy outcomes. BMC Endocr Disord 2014; $14: 47$.

60. Hyperglycaemia and Adverse Pregnancy Outcome (HAPO) Study: associations with maternal body mass index. BJOG 2010; 117(5):575-84.

61. Isaacs JD, Magann EF, Martin RW, Chauhan SP, Morrison JC. Obstetric challenges of massive obesity complicating pregnancy. J Perinatol 1994; 14(1):10-4.

62. Hollowell J, Pillas D, Rowe R, Linsell L, Knight M, Brocklehurst P. The impact of maternal obesity on intrapartum outcomes in otherwise low risk women: secondary analysis of the Birthplace national prospective cohort study. BJOG 2014; 121(3):343-55.

63. Garbaciak JA, JR, Richter M, Miller S, Barton JJ. Maternal weight and pregnancy complications. American Journal of Obstetrics and Gynecology 1985; 152(2):238-45.

64. Stepan H, Scheithauer S, Dornhofer N, Kramer T, Faber R. Obesity as an obstetric risk factor: does it matter in a perinatal center? Obesity (Silver Spring) 2006; 14(5):770-3.

65. Young TK, Woodmansee B. Factors that are associated with cesarean delivery in a large private practice: The importance of prepregnancy body mass index and weight gain. American Journal of Obstetrics and Gynecology 2002; 187(2):312-20.

66. Chu SY, Kim SY, Schmid CH, Dietz PM, Callaghan WM, Lau J et al. Maternal obesity and risk of cesarean delivery: A meta-analysis. Obesity Reviews 2007; 8(5):385-94.

67. Arrowsmith S, Wray S, Quenby S. Maternal obesity and labour complications following induction of labour in prolonged pregnancy. BJOG: An International Journal of Obstetrics \& Gynaecology 2011; 118(5):578-88. 
68. Vahratian A, Zhang J, Troendle JF, Savitz DA, Siega-Riz AM. Maternal Prepregnancy Overweight and Obesity and the Pattern of Labor Progression in Term Nulliparous Women. Obstetrics \& Gynecology 2004; 104(5, Part 1):943-51.

69. Rode L, Nilas L, Wojdemann K, Tabor A. Obesity-related complications in Danish single cephalic term pregnancies. Obstet Gynecol 2005; 105(3):537-42.

70. Heslehurst N, Simpson H, Ells LJ, Rankin J, Wilkinson J, Lang R et al. The impact of maternal BMI status on pregnancy outcomes with immediate short-term obstetric resource implications: A meta-analysis. Obesity Reviews 2008; 9(6):635-83.

71. Magann EF, Doherty DA, Chauhan SP, Klimpel JM, Huff SD, Morrison JC. Pregnancy, obesity, gestational weight gain, and parity as predictors of peripartum complications. Arch Gynecol Obstet 2011; 284(4):827-36.

72. Weiss JL, Malone FD, Emig D, Ball RH, Nyberg DA, Comstock CH et al. Obesity, obstetric complications and cesarean delivery rate--a population-based screening study. American Journal of Obstetrics and Gynecology 2004; 190(4):1091-7.

73. Raisanen S, Gissler M, Saari J, Kramer M, Heinonen S. Contribution of risk factors to extremely, very and moderately preterm births - register-based analysis of 1,390,742 singleton births. PLoS One 2013; 8(4):e60660.

74. Cedergren MI. Maternal morbid obesity and the risk of adverse pregnancy outcome. Obstet Gynecol 2004; 103(2):219-24.

75. Cnattingius S, Villamor E, Johansson S, Edstedt Bonamy A-K, Persson M, Wikström A-K et al. Maternal obesity and risk of preterm delivery. JAMA 2013; 309(22):2362-70.

76. Zhang J, Bricker L, Wray S, Quenby S. Poor uterine contractility in obese women. BJOG 2007; 114(3):343-8.

77. Mehta SH, Blackwell SC, Bujold E, Sokol RJ. What factors are associated with neonatal injury following shoulder dystocia? J Perinatol 2006; 26(2):85-8.

78. Baskett TF. Shoulder dystocia. Best Pract Res Clin Obstet Gynaecol 2002; 16(1):57-68.

79. Ginsberg NA, Moisidis C. How to predict recurrent shoulder dystocia. American Journal of Obstetrics and Gynecology 2001; 184(7):1427-9; discussion 1429-30. 
80. Robinson H, Tkatch S, Mayes DC, Bott N, Okun N. Is maternal obesity a predictor of shoulder dystocia? Obstet Gynecol 2003; 101(1):24-7.

81. Bianco AT, Smilen SW, Davis Y, Lopez S, Lapinski R, Lockwood CJ. Pregnancy outcome and weight gain recommendations for the morbidly obese woman. Obstet Gynecol 1998; 91(1):97-102.

82. Johnson JW, Longmate JA, Frentzen B. Excessive maternal weight and pregnancy outcome. American Journal of Obstetrics and Gynecology 1992; 167(2):353-70; discussion $370-2$.

83. Blomberg M. Maternal Obesity and Risk of Postpartum Hemorrhage. Obstetrics \& Gynecology 2011; 118(3):561-8.

84. Sewell MF, Huston-Presley L, Super DM, Catalano P. Increased neonatal fat mass, not lean body mass, is associated with maternal obesity. American Journal of Obstetrics and Gynecology 2006; 195(4):1100-3.

85. Gaudet L, Ferraro ZM, Wen SW, Walker M. Maternal obesity and occurrence of fetal macrosomia: a systematic review and meta-analysis. Biomed Res Int 2014; 2014:640291.

86. Zhu T, Tang J, Zhao F, Qu Y, Mu D. Association between maternal obesity and offspring Apgar score or cord pH: a systematic review and meta-analysis. Sci Rep 2015; 5:18386.

87. Persson M, Johansson S, Villamor E, Cnattingius S. Maternal overweight and obesity and risks of severe birth-asphyxia-related complications in term infants: a population-based cohort study in Sweden. PLoS Med 2014; 11(5):e1001648.

88. Queisser-Luft A, Kieninger-Baum D, Menger H, Stolz G, Schlaefer K, Merz E. Erhöht mütterliche Adipositas das Risiko fur kindliche Fehlbildungen? Analyse von 20,248 Neugeborenen des Mainzer Geburtenregisters zur Erfassung angeborener Fehlbildungen. Ultraschall Med 1998; 19(1):40-4.

89. Shaw GM, Velie EM, Schaffer D. Risk of neural tube defect-affected pregnancies among obese women. JAMA 1996; 275(14):1093-6.

90. Shaw GM, Todoroff K, Schaffer DM, Selvin S. Maternal height and prepregnancy body mass index as risk factors for selected congenital anomalies. Paediatr Perinat Epidemiol 2000; 14(3):234-9. 
91. Waller DK, Mills JL, Simpson JL, Cunningham GC, Conley MR, Lassman MR et al. Are obese women at higher risk for producing malformed offspring? American Journal of Obstetrics and Gynecology 1994; 170(2):541-8.

92. Waller DK, Shaw GM, Rasmussen SA, Hobbs CA, Canfield MA, Am Siega-Riz et al. Prepregnancy obesity as a risk factor for structural birth defects. Arch Pediatr Adolesc Med 2007; 161(8):745-50.

93. Anderson JL, Waller DK, Canfield MA, Shaw GM, Watkins ML, Werler MM. Maternal obesity, gestational diabetes, and central nervous system birth defects. Epidemiology 2005; 16(1):87-92.

94. Stothard KJ, Tennant PWG, Bell R, Rankin J. Maternal overweight and obesity and the risk of congenital anomalies: a systematic review and meta-analysis. JAMA 2009; 301(6):636-50.

95. Cedergren M, Kallen B. Maternal obesity and the risk for orofacial clefts in the offspring. Cleft Palate Craniofac J 2005; 42(4):367-71.

96. Cedergren MI, Kallen BAJ. Maternal obesity and infant heart defects. Obes Res 2003; 11(9):1065-71.

97. Tennant PWG, Rankin J, Bell R. Maternal body mass index and the risk of fetal and infant death: a cohort study from the North of England. Hum Reprod 2011; 26(6):1501-11.

98. Getahun D, Ananth CV, Kinzler WL. Risk factors for antepartum and intrapartum stillbirth: a population-based study. American Journal of Obstetrics and Gynecology 2007; 196(6):499-507.

99. Stephansson O, Dickman PW, Johansson A, Cnattingius S. Maternal weight, pregnancy weight gain, and the risk of antepartum stillbirth. American Journal of Obstetrics and Gynecology 2001; 184(3):463-9.

100. Hilson JA, Rasmussen KM, Kjolhede CL. High prepregnant body mass index is associated with poor lactation outcomes among white, rural women independent of psychosocial and demographic correlates. J Hum Lact 2004; 20(1):18-29.

101. Guelinckx I, Devlieger R, Bogaerts A, Pauwels S, Vansant G. The effect of pre-pregnancy BMI on intention, initiation and duration of breast-feeding. Public Health Nutr 2012; 15(5):840-8. 
102. Liu J, Smith MG, Dobre MA, Ferguson JE. Maternal obesity and breast-feeding practices among white and black women. Obesity (Silver Spring) 2010; 18(1):175-82.

103. Turcksin R, Bel S, Galjaard S, Devlieger R. Maternal obesity and breastfeeding intention, initiation, intensity and duration: a systematic review. Matern Child Nutr 2014; 10(2):166-83.

104. Schaefer-Graf UM, Hartmann R, Pawliczak J, Passow D, Abou-Dakn M, Vetter K et al. Association of breast-feeding and early childhood overweight in children from mothers with gestational diabetes mellitus. Diabetes Care 2006; 29(5):1105-7.

105. Myles TD, Gooch J, Santolaya J. Obesity as an independent risk factor for infectious morbidity in patients who undergo cesarean delivery. Obstet Gynecol 2002; 100(5 Pt 1):959-64.

106. Konje JC, Imrie A, Hay DM. Pregnancy in obese women. Journal of Obstetrics and Gynaecology 1993; 13(6):413-8.

107. Gunderson EP, Jacobs DR, JR, Chiang V, Lewis CE, Feng J, Quesenberry CP, JR et al. Duration of lactation and incidence of the metabolic syndrome in women of reproductive age according to gestational diabetes mellitus status: a 20-Year prospective study in CARDIA (Coronary Artery Risk Development in Young Adults). Diabetes 2010; 59(2):495504.

108. Schwarz EB, Ray RM, Stuebe AM, Allison MA, Ness RB, Freiberg MS et al. Duration of lactation and risk factors for maternal cardiovascular disease. Obstet Gynecol 2009; 113(5):974-82.

109. Liu B, Jorm L, Banks E. Parity, breastfeeding, and the subsequent risk of maternal type 2 diabetes. Diabetes Care 2010; 33(6):1239-41.

110. Stuebe AM, Rich-Edwards JW, Willett WC, Manson JE, Michels KB. Duration of lactation and incidence of type 2 diabetes. JAMA 2005; 294(20):2601-10.

111. Krause KM, Lovelady CA, Peterson BL, Chowdhury N, Ostbye T. Effect of breast-feeding on weight retention at 3 and 6 months postpartum: data from the North Carolina WIC Programme. Public Health Nutr 2010; 13(12):2019-26. 
112. Breast cancer and breastfeeding: collaborative reanalysis of individual data from 47 epidemiological studies in 30 countries, including 50302 women with breast cancer and 96973 women without the disease: Collaborative reanalysis of individual data from 47 epidemiological studies in 30 countries, including 50302 women with breast cancer and 96 973 women without the disease. Lancet 2002; 360(9328):187-95.

113. Sung HK, Ma SH, Choi J-Y, Hwang Y, Ahn C, Kim B-G et al. The Effect of Breastfeeding Duration and Parity on the Risk of Epithelial Ovarian Cancer: A Systematic Review and Meta-analysis. J Prev Med Public Health 2016; 49(6):349-66.

114. Grube MM, Lippe E von der, Schlaud M, Brettschneider A-K. Does breastfeeding help to reduce the risk of childhood overweight and obesity? A propensity score analysis of data from the KiGGS study. PLoS One 2015; 10(3):e0122534.

115. Heinig MJ. Host defense benefits of breastfeeding for the infant. Effect of breastfeeding duration and exclusivity. Pediatr Clin North Am 2001; 48(1):105-23, ix.

116. Chen A, Rogan WJ. Breastfeeding and the risk of postneonatal death in the United States. Pediatrics 2004; 113(5):e435-9.

117. Gdalevich M, Mimouni D, Mimouni M. Breast-feeding and the risk of bronchial asthma in childhood: a systematic review with meta-analysis of prospective studies. J Pediatr 2001; 139(2):261-6.

118. Horwood LJ, Darlow BA, Mogridge N. Breast milk feeding and cognitive ability at 7-8 years. Arch Dis Child Fetal Neonatal Ed 2001; 84(1):F23-7.

119. Weissenborn A, Abou-Dakn M, Bergmann R, Both D, Gresens R, Hahn B et al. Stillhaufigkeit und Stilldauer in Deutschland - eine systematische Übersicht. Gesundheitswesen (Bundesverband der Ärzte des Öffentlichen Gesundheitsdienstes (Germany)) 2016; 78(11):695-707.

120. Forsen T, Eriksson JG, Tuomilehto J, Teramo K, Osmond C, Barker DJ. Mother's weight in pregnancy and coronary heart disease in a cohort of Finnish men: follow up study. BMJ 1997; 315(7112):837-40.

121. Fall CH, Stein CE, Kumaran K, Cox V, Osmond C, Barker DJ et al. Size at birth, maternal weight, and type 2 diabetes in South India. Diabet Med 1998; 15(3):220-7. 
122. Li Y-M, Ou J-J, Liu L, Zhang D, Zhao J-P, Tang S-Y. Association Between Maternal Obesity and Autism Spectrum Disorder in Offspring: A Meta-analysis. J Autism Dev Disord 2016; 46(1):95-102.

123. Pugh SJ, Richardson GA, Hutcheon JA, Himes KP, Brooks MM, Day NL et al. Maternal Obesity and Excessive Gestational Weight Gain Are Associated with Components of Child Cognition. J Nutr 2015; 145(11):2562-9.

124. Forno E, Young OM, Kumar R, Simhan H, Celedon JC. Maternal obesity in pregnancy, gestational weight gain, and risk of childhood asthma. Pediatrics 2014; 134(2):e535-46.

125. Rodriguez A, Miettunen J, Henriksen TB, Olsen J, Obel C, Taanila A et al. Maternal adiposity prior to pregnancy is associated with ADHD symptoms in offspring: evidence from three prospective pregnancy cohorts. Int J Obes (Lond) 2008; 32(3):550-7.

126. Gaillard R, Felix JF, Duijts L, Jaddoe VWV. Childhood consequences of maternal obesity and excessive weight gain during pregnancy. Acta Obstet Gynecol Scand 2014; 93(11):1085-9.

127. Gaillard R. Maternal obesity during pregnancy and cardiovascular development and disease in the offspring. Eur J Epidemiol 2015; 30(11):1141-52.

128. Gaillard R, Santos S, Duijts L, Felix JF. Childhood Health Consequences of Maternal Obesity during Pregnancy: A Narrative Review. Ann Nutr Metab 2016; 69(3-4):171-80.

129. Yu Z, Han S, Zhu J, Sun X, Ji C, Guo X. Pre-pregnancy body mass index in relation to infant birth weight and offspring overweight/obesity: a systematic review and meta-analysis. PLoS One 2013; 8(4):e61627.

130. Ramsay JE, Ferrell WR, Crawford L, Am Wallace, Greer IA, Sattar N. Maternal obesity is associated with dysregulation of metabolic, vascular, and inflammatory pathways. J Clin Endocrinol Metab 2002; 87(9):4231-7.

131. Gaillard R, Rifas-Shiman SL, Perng W, Oken E, Gillman MW. Maternal inflammation during pregnancy and childhood adiposity. Obesity (Silver Spring) 2016; 24(6):1320-7.

132. Leiva A, Pardo F, Ramirez MA, Farias M, Casanello P, Sobrevia L. Fetoplacental vascular endothelial dysfunction as an early phenomenon in the programming of human adult diseases in subjects born from gestational diabetes mellitus or obesity in pregnancy. Exp Diabetes Res 2011; 2011:349286. 
133. Plagemann A. 'Fetal programming' and 'functional teratogenesis': on epigenetic mechanisms and prevention of perinatally acquired lasting health risks. J Perinat Med 2004; 32(4):297-305.

134. Plagemann A. A matter of insulin: developmental programming of body weight regulation. J Matern Fetal Neonatal Med 2008; 21(3):143-8.

135. Schellong K, Rodekamp E, Harder T, Dudenhausen J, Plagemann A. Geburtsgewicht, Gestationsdiabetes und Perinatale Programmierung. Gynakol Geburtsmed Gynakol Endokrinol 2009; (5(3)):182-92.

136. Groom A, Elliott HR, Embleton ND, Relton CL. Epigenetics and child health: basic principles. Arch Dis Child 2011; 96(9):863-9.

137. Dorner G, Plagemann A. Perinatal hyperinsulinism as possible predisposing factor for diabetes mellitus, obesity and enhanced cardiovascular risk in later life. Horm Metab Res 1994; 26(5):213-21.

138. Lucas A, Sarson DL, Blackburn AM, Adrian TE, Aynsley-Green A, Bloom SR. Breast vs bottle: endocrine responses are different with formula feeding. Lancet 1980; 1(8181):1267-9.

139. Deutsche Gesellschaft für Gynäkologie und Geburtshilfe (DGGG), Österreichische Gesellschaft für Gynäkologie und Geburtshilfe (OEGGG), Schweizerische Gesellschaft für Gynäkologie und Geburtshilfe (SGGG). Peripartale Blutungen, Diagnostik und Therapie; Klasse S2k: Arbeitsgemeinschaft der Wissenschaftlichen Medizinischen Fachgesellschaften (AWMF); 2016 015/063.

140. Voigt M, Schneider KT, Jahrig K. Analysis of a 1992 birth sample in Germany. 1: New percentile values of the body weight of newborn infants. Geburtshilfe Frauenheilkd 1996; 56(10):550-8.

141. Rasmussen KM, Abrams B, Bodnar LM, Butte NF, Catalano PM, Maria S-RA. Recommendations for weight gain during pregnancy in the context of the obesity epidemic. Obstet Gynecol 2010; 116(5):1191-5.

142. Apgar V. A Proposal for a New Method of Evaluation of the Newborn Infant. Originally published in July 1953, volume 32, pages 250-259. Anesth Analg 2015; 120(5):1056-9. 
143. Reiss K, Breckenkamp J, Borde T, Brenne S, David M, Razum O. Contribution of overweight and obesity to adverse pregnancy outcomes among immigrant and non-immigrant women in Berlin, Germany. Eur J Public Health 2015; 25(5):839-44.

144. Rosenn B, Miodovnik M, Combs CA, Khoury J, Siddiqi TA. Glycemic thresholds for spontaneous abortion and congenital malformations in insulin-dependent diabetes mellitus. Obstet Gynecol 1994; 84(4):515-20.

145. Combs CA, Kitzmiller JL. Spontaneous abortion and congenital malformations in diabetes. Baillieres Clin Obstet Gynaecol 1991; 5(2):315-31.

146. Kumari AS. Pregnancy outcome in women with morbid obesity. Int J Gynaecol Obstet 2001; 73(2):101-7.

147. Crane JMG, White J, Murphy P, Burrage L, Hutchens D. The effect of gestational weight gain by body mass index on maternal and neonatal outcomes. J Obstet Gynaecol Can 2009; 31(1):28-35.

148. Kiel DW, Dodson EA, Artal R, Boehmer TK, Leet TL. Gestational weight gain and pregnancy outcomes in obese women: how much is enough? Obstet Gynecol 2007; 110(4):752-8.

149. Wolff S, Legarth J, Vangsgaard K, Toubro S, Astrup A. A randomized trial of the effects of dietary counseling on gestational weight gain and glucose metabolism in obese pregnant women. Int J Obes (Lond) 2008; 32(3):495-501.

150. Chu SY, Bachman DJ, Callaghan WM, Whitlock EP, Dietz PM, Berg CJ et al. Association between obesity during pregnancy and increased use of health care. N Engl J Med 2008; 358(14):1444-53.

151. Galtier-Dereure F, Boegner C, Bringer J. Obesity and pregnancy: complications and cost. Am J Clin Nutr 2000; 71(5 Suppl):1242S-8S.

152. Knight M, Nair M, Tuffnell D, Kenyon S, Shakespeare J, Brocklehurst P et al. Saving Lives, Improving Mothers' Care - Surveillance of maternal deaths in the UK 2012-14 and lessons learned to inform maternity care from the UK and Ireland Confidential Enquiries into Maternal Deaths and Morbidity 2009-14: National Perinatal Epidemiology Unit, University of Oxford; 2016. 
153. Kleinwechter H, Schäfer-Graf U, Bührer C, Hoesli I, Kainer F, Kautzky-Willer A et al. S3-Leitlinie Gestationsdiabetes mellitus (GDM), Diagnostik, Therapie und Nachsorge der Deutschen Diabetes Gesellschaft (DDG) und der Deutschen Gesellschaft für Gynäkologie und Geburtshilfe (DGGG): 2. Auflage: Arbeitsgemeinschaft der Wissenschaftlichen Medizinischen Fachgesellschaften (AWMF); 2018 057/008.

154. Langer O, Yogev Y, Xenakis EMJ, Brustman L. Overweight and obese in gestational diabetes: the impact on pregnancy outcome. American Journal of Obstetrics and Gynecology 2005; 192(6):1768-76.

155. Crowther CA, Hiller JE, Moss JR, McPhee AJ, Jeffries WS, Robinson JS. Effect of treatment of gestational diabetes mellitus on pregnancy outcomes. N Engl J Med 2005; 352(24):2477-86.

156. Landon MB, Spong CY, Thom E, Carpenter MW, Ramin SM, Casey B et al. A multicenter, randomized trial of treatment for mild gestational diabetes. N Engl J Med 2009; 361(14):1339-48.

157. Hunger-Dathe W, Mosebach N, Samann A, Wolf G, Muller UA. Prevalence of impaired glucose tolerance 6 years after gestational diabetes. Exp Clin Endocrinol Diabetes 2006; 114(1):11-7.

158. Vest AR, Cho LS. Hypertension in pregnancy. Curr Atheroscler Rep 2014; 16(3):395.

159. Steegers EA, Dadelszen P von, Duvekot JJ, Pijnenborg R. Pre-eclampsia. Lancet 2010; 376(9741):631-44.

160. Scholl TO, Chen X, Goldberg GS, Khusial PR, Stein TP. Maternal diet, C-reactive protein, and the outcome of pregnancy. J Am Coll Nutr 2011; 30(4):233-40.

161. Qiu C, Luthy DA, Zhang C, Walsh SW, Leisenring WM, Williams MA. A prospective study of maternal serum C-reactive protein concentrations and risk of preeclampsia. Am J Hypertens 2004; 17(2):154-60.

162. Wolf M, Kettyle E, Sandler L, Ecker JL, Roberts J, Thadhani R. Obesity and preeclampsia: the potential role of inflammation. Obstet Gynecol 2001; 98(5 Pt 1):757-62. 
163. Palei ACT, Sandrim VC, Cavalli RC, Tanus-Santos JE. Comparative assessment of matrix metalloproteinase (MMP)-2 and MMP-9, and their inhibitors, tissue inhibitors of metalloproteinase (TIMP)-1 and TIMP-2 in preeclampsia and gestational hypertension. Clin Biochem 2008; 41(10-11):875-80.

164. Wolf M, Sandler L, Munoz K, Hsu K, Ecker JL, Thadhani R. First trimester insulin resistance and subsequent preeclampsia: a prospective study. J Clin Endocrinol Metab 2002; 87(4):1563-8.

165. Bienertova-Vasku J, Dostalova Z, Kankova K, Bienert P, Vasku A, Unzeitig V. Is there any link between severe pre-eclampsia and defined polymorphisms in leptin and adiponectin genes? J Obstet Gynaecol Res 2008; 34(5):858-64.

166. Dalamaga M, Srinivas SK, Elovitz MA, Chamberland J, Mantzoros CS. Serum adiponectin and leptin in relation to risk for preeclampsia: results from a large case-control study. Metabolism 2011; 60(11):1539-44.

167. Xiong X, Wang FL, Davidge ST, Demianczuk NN, Mayes DC, Olson DM et al. Maternal smoking and preeclampsia. J Reprod Med 2000; 45(9):727-32.

168. Cox JL, Holden JM, Sagovsky R. Detection of postnatal depression. Development of the 10-item Edinburgh Postnatal Depression Scale. Br J Psychiatry 1987; 150:782-6.

169. Doherty DA, Magann EF, Francis J, Morrison JC, Newnham JP. Pre-pregnancy body mass index and pregnancy outcomes. Int J Gynaecol Obstet 2006; 95(3):242-7.

170. Kramer MS, Platt R, Yang H, McNamara H, Usher RH. Are all growth-restricted newborns created equal(ly)? Pediatrics 1999; 103(3):599-602.

171. Radulescu L, Munteanu O, Popa F, Cirstoiu M. The implications and consequences of maternal obesity on fetal intrauterine growth restriction. J Med Life 2013; 6(3):292-8.

172. Marchi J, Berg M, Dencker A, Olander EK, Begley C. Risks associated with obesity in pregnancy, for the mother and baby: A systematic review of reviews. Obes Rev 2015; 16(8):621-38.

173. Schäfer-Graf U, Gembruch U, Louwen F, Schmidt M. Adipositas und Schwangerschaft. Der Frauenarzt 2017; 58(1):22-7. 
174. Smith RD, Babiychuk EB, Noble K, Draeger A, Wray S. Increased cholesterol decreases uterine activity: functional effects of cholesterol alteration in pregnant rat myometrium. Am J Physiol Cell Physiol 2005; 288(5):C982-8.

175. Gimpl G, Fahrenholz F. Human oxytocin receptors in cholesterol-rich vs. cholesterolpoor microdomains of the plasma membrane. Eur J Biochem 2000; 267(9):2483-97.

176. Catalano PM, Hauguel-De Mouzon S. Is it time to revisit the Pedersen hypothesis in the face of the obesity epidemic? American Journal of Obstetrics and Gynecology 2011; 204(6):479-87.

177. Schaefer-Graf UM, Graf K, Kulbacka I, Kjos SL, Dudenhausen J, Vetter K et al. Maternal lipids as strong determinants of fetal environment and growth in pregnancies with gestational diabetes mellitus. Diabetes Care 2008; 31(9):1858-63.

178. Feldman B, Yaron Y, Critchfield G, Leon J, O'Brien JE, Johnson MP et al. Distribution of neural tube defects as a function of maternal weight: no apparent correlation. Fetal Diagn Ther 1999; 14(3):185-9.

179. Lam PK, Torfs CP, Brand RJ. A low pregnancy body mass index is a risk factor for an offspring with gastroschisis. Epidemiology 1999; 10(6):717-21.

180. Ashoor G, Syngelaki A, Poon LCY, Rezende JC, Nicolaides KH. Fetal fraction in maternal plasma cell-free DNA at 11-13 weeks' gestation: relation to maternal and fetal characteristics. Ultrasound Obstet Gynecol 2013; 41(1):26-32.

181. Dashe JS, McIntire DD, Twickler DM. Effect of maternal obesity on the ultrasound detection of anomalous fetuses. Obstet Gynecol 2009; 113(5):1001-7.

182. Chu SY, Kim SY, Lau J, Schmid CH, Dietz PM, Callaghan WM et al. Maternal obesity and risk of stillbirth: a metaanalysis. American Journal of Obstetrics and Gynecology 2007; 197(3):223-8.

183. Salihu HM, Dunlop AL, Hedayatzadeh M, Alio AP, Kirby RS, Alexander GR. Extreme obesity and risk of stillbirth among black and white gravidas. Obstet Gynecol 2007; 110(3):552-7.

184. Yao R, Ananth CV, Park BY, Pereira L, Plante LA. Obesity and the risk of stillbirth: A population-based cohort study. American Journal of Obstetrics and Gynecology 2014; 210(5):457.e1-457.e9. 
185. Pinar H, Goldenberg RL, Koch MA, Heim-Hall J, Hawkins HK, Shehata B et al. Placental findings in singleton stillbirths. Obstet Gynecol 2014; 123(2 Pt 1):325-36.

186. Amir LH, Donath SM. Socioeconomic status and rates of breastfeeding in Australia: evidence from three recent national health surveys. Med J Aust 2008; 189(5):254-6.

187. Rauh K, Gabriel E, Kerschbaum E, Schuster T, Kries R von, Amann-Gassner U et al. Safety and efficacy of a lifestyle intervention for pregnant women to prevent excessive maternal weight gain: a cluster-randomized controlled trial. BMC Pregnancy Childbirth 2013; $13: 151$.

188. Thangaratinam S, Rogozinska E, Jolly K, Glinkowski S, Roseboom T, Tomlinson JW et al. Effects of interventions in pregnancy on maternal weight and obstetric outcomes: metaanalysis of randomised evidence. BMJ 2012; 344:e2088.

189. Magro-Malosso ER, Saccone G, Di Mascio D, Di Tommaso M,. Exercise during pregnancy and risk of preterm birth in overweight and obese women: a systematic review and meta-analysis of randomized controlled trials. Acta Obstet Gynecol Scand 2016.

190. Rauh K, Kunath J, Rosenfeld E, Kick L, Ulm K, Hauner H. Healthy living in pregnancy: a cluster-randomized controlled trial to prevent excessive gestational weight gain - rationale and design of the GeliS study. BMC Pregnancy Childbirth 2014; 14:119.

191. Kunath J, Günther J, Rauh K, Hoffmann J, Stecher L, Rosenfeld E et al. Effects of a lifestyle intervention during pregnancy to prevent excessive gestational weight gain in routine care - the cluster-randomised GeliS trial. BMC Med 2019; 17(1):5.

192. Günther J, Hoffmann J, Kunath J, Spies M, Meyer D, Stecher L et al. Effects of a Lifestyle Intervention in Routine Care on Prenatal Dietary Behavior-Findings from the ClusterRandomized GeliS Trial. J Clin Med 2019; 8(7).

193. Hoffmann J, Günther J, Stecher L, Spies M, Meyer D, Kunath J et al. Effects of a Lifestyle Intervention in Routine Care on Short- and Long-Term Maternal Weight Retention and Breastfeeding Behavior-12 Months Follow-up of the Cluster-Randomized GeliS Trial. J Clin Med 2019; 8(6).

194. Bundesministerium für Ernährung und Landwirtschaft, Kompetenzzentrum für Ernährung (KErn), Netzwerk Gesund ins Leben, Stiftung Kindergesundheit. Die App-Trilogie: Für Schwangere und junge Familien zu den Themen gesunde Ernährung und Bewegung [cited 2019 Aug 7]. Available from: URL: https://www.familie-gesund-ernaehrt.de/. 
Mein besonderer Dank gilt Frau Prof. Dr. med. Birgit Seelbach-Göbel, Direktorin und Chefärztin der Klinik für Frauenheilkunde und Geburtshilfe sowie Lehrstuhlinhaberin der Universität Regensburg, für die Überlassung des interessanten Themas und die hervorragende Betreuung während der gesamten Bearbeitungsphase der Dissertation. Ihr Vertrauen, ihre - durch ein hohes Maß an Kompetenz und Erfahrung gekennzeichnete - Diskussions- und Hilfsbereitschaft, ihre Anregungen sowie ihre Verbesserungsvorschläge haben mich stets motiviert und wesentlich zum Gelingen dieser Arbeit beigetragen.

Danksagen möchte ich auch Herrn Florian Zeman, stellvertretender Leiter des Zentrums für klinische Studien der Universität Regensburg, für seine fachkundige Beratung und Unterstützung bei der Erstellung des zu untersuchenden Datensatzes und der statistischen Auswertung.

Herrn Dr. Prager und Frau Kostka gilt mein Dank für die Hilfe bei der Rekrutierung der benötigten Patientendaten aus der Kreißsaal-Datenbank und dem Patientenakten-Archiv.

Dem Sekretariat und dem gesamten Team der Ambulanz der Klinik für Frauenheilkunde und Geburtshilfe St. Hedwig, insbesondere Frau Scheuerer und Frau Heyn, bin ich für das uneingeschränkt freundliche Entgegenkommen und für die Flexibilität bei der Terminorganisation dankbar.

Für die zuverlässige und hilfreiche Unterstützung bei der Beschaffung einzelner, schwer zugänglicher Volltexte zu wissenschaftlichen Veröffentlichungen möchte ich außerdem Frau Saha meinen Dank aussprechen. 


\section{EIDESSTATTLICHE ERKLÄRUNG}

Hiermit erkläre ich an Eides Statt, dass ich die vorliegende Arbeit selbstständig, ohne unzulässige Hilfe Dritter und ohne Benutzung anderer als der angegebenen Hilfsmittel angefertigt habe. Die aus anderen Quellen direkt oder indirekt übernommenen Daten und Konzepte sind unter Angabe der Quelle gekennzeichnet.

Insbesondere habe ich nicht die entgeltliche Hilfe von Vermittlungs- und Beratungsdiensten in Anspruch genommen. Niemand hat von mir unmittelbar oder mittelbar geldwerte Leistungen für Arbeit erhalten, die im Zusammenhang mit dem Inhalt der vorgelegten Dissertation stehen.

Diese Arbeit wurde bisher weder im In- noch im Ausland in gleicher oder ähnlicher Form einer anderen Prüfungsbehörde vorgelegt.

Ich erkläre, dass mir keine Tatsachen bekannt sind, die mich zur Führung eines akademischen Grades im Sinne des Gesetzes über die Führung akademischer Grade unwürdig erscheinen lassen.

Regensburg, den

Susanne Artus 
